\title{
Development and application of otolith- based methods to infer demographic connections in a marine metapopulation
}

by

Philipp Neubauer

\author{
A thesis submitted to \\ Victoria University of Wellington \\ in fulfilment of the requirements for the degree of \\ Doctor of Philosophy in Marine Biology
}

Victoria University of Wellington

2012 

This thesis has been conducted under the supervision of:

Dr. Jeffrey S. Shima (Primary Supervisor)

Victoria University of Wellington

Wellington, New Zealand

and

Dr. Steven E. Swearer (Secondary Supervisor)

University of Melbourne

Melbourne, Australia 

To my family,

for past, present

and future. 


\section{Abstract}

Connectivity between local populations is critical if these are to function as a metapopulation and sustain locally open sink populations. Assessing whether such connections between local populations exist is thus an important step towards understanding coastal metapopulation dynamics as well as assessing the efficacy of spatial management tools such as marine reserve networks. For this thesis, I investigate population connectivity of the common triplefin (Forsterygion lapillum) in Cook Strait, New Zealand, using chemical signatures contained within fish otoliths (ear stones). I concentrate on likely connections between three local marine reserves: Kapiti Island (Kapiti coast), Long Island (Marlborough Sounds) and Taputeranga Marine Reserve (Wellington south coast). To this end I develop and implement new statistical methods to enable stronger inferences from otolith chemistry based approaches.

In chapter 2, I evaluate otolith core chemistry as a potential tool (i.e. an environmental fingerprint) for identification of natal source populations of the common triplefin. I sampled otolith chemistry from hatchling fish across a range of hierarchical scales: obtained from individual egg masses within a site; sites within different regions; and regions distributed on the two main islands of New Zealand (North and South Island). This sampling enabled me to construct an "atlas" (or baseline) of otolith core chemistry. I developed and applied a set of novel statistical approaches to examine the characteristics of this natal atlas and optimize its spatial resolution. These analyses allowed me to assess the utility of otolith chemistry as a potential tool to infer patterns of population connectivity in the vicinity of Cook Strait.

Chapter 3 develops a new Bayesian approach to facilitate improved clustering and classification of dispersing fish to putative natal populations based on their otolith chemistry. Otolith-based approaches used to infer 
natal origins of fishes routinely suffer from the (unrealized) requirement to sample all potential natal source populations. An incomplete baseline atlas has greatly limited the application of otolith chemistry as a tool for assessments of connectivity in the marine environment. In this chapter, I develop, evaluate, and implement statistical solutions to this problem. Specifically, I present a clustering model, based on infinite mixtures, which does not require the specification of a potential number of sources. In a second step, I embed this clustering model in a large-scale classification model that allows for classification on scales encompassing a number of potential sources, where recruits are clustered with observations from the baseline or a separate cluster within these regions. This opens the potential for fish that came from an identifiable source other than those sampled to not be assigned to a sampled source. I evaluate the strength of this approach using the well-known weakfish (Cynoscion regalis) dataset.

In chapter 4, I apply the statistical methods developed in chapter 3 to the common triplefin. I sampled recent recruits of the common triplefin within each of three marine reserves (Kapiti, Long Island, and Taputeranga) and used otolith chemistry to infer probable natal origins. I then compare these inferred patterns of connectivity with those predicted by a set of hydrodynamic simulations. This comparison enabled me to (qualitatively) assess the likelihood of connectivity (as predicted by otolith chemistry) given local hydrodynamic conditions.

For chapter 5, I extend the Bayesian modelling approaches developed in previous chapters to incorporate otolith chemistry data sampled from throughout the life-history of dispersers. As in chapter 3, I develop and evaluate the utility of this approach using a previously published data set (Chinook salmon), and I apply the approach to the common triplefin in a subsequent chapter. Specifically, I propose flexible formulations based on latent state models, and compare these in a series of illustrative simulations and an application to Chinook salmon contingent analysis. 
In chapter 6, I apply the Bayesian framework (developed in chapter 5) to the common triplefin data set. Specifically, I formulate a model based on putative chemical distinctions between inshore and offshore watermasses. This model allows me to compare dispersal histories among recruits to a set of reserves (evaluated initially in chapter 4), and the approach reveals patterns that appear to be common to all successful recruits. I examine these findings in the light of results obtained in chapter 4 as well as local hydrodynamic conditions.

Finally, I conclude my thesis in chapter 7 by discussing the relevance of my findings for the functioning of networks of sub-populations, both in a metapopulation and a reserve network context. 


\section{Acknowledgements}

I thank my supervisors, Jeff Shima and Steve Swearer for giving me the opportunity to pursue my $\mathrm{PhD}$, and for providing valuable and critical assessment of my work, which helped me to develop my thinking and writing in important ways.

This work wouldn't have been possible without the continuous and input and help from my dear friends and colleagues at the Victoria University Coastal Ecology Laboratory, and I wish to especially thank Alejandro Perez-Matus for providing initial support when I first started out and for sharing his knowledge of the system and of fish biology in general.

The statistical developments in this thesis were influenced and supported by a number of friends and colleagues. I especially thank Sergio Hernandez for introducing me to mixture models and for discussing geeky concepts such as fuzzy clustering and Dirichlet processes with me. My visit with Babak Shababah helped me further develop these ideas, and I thank him for his hospitality. Discussions with Michele Masuda, Russel Millar, as well as with Jessica Miller and her lab-group at Oregon State University proved useful in advancing the work presented in this thesis. I also thank Jessica Miller and Simon Thorrold for sharing their data with me.

I am grateful to Shirley Pledger, Richard Arnold and Marcus Frean for letting me sit in with their respective statistics and machine learning classes, which helped me to make connections that I would not have made otherwise. I also thank Marti Krcoçek and Mark Lewis for accepting me on their ecological modelling course at Bamfield - the discussions with this smart and fun group were invaluable. I am indebted to Bruce Menge and the Pisco team at OSU for hosting me for extended periods and providing me with a welcoming and enriching environment to work on this thesis. 
I am incredibly thankful to my amazing wife and love of my life, Emilie Fleur, for making this the most eventful and unforgettable time of my life. Her support and criticism are my drive to become a better scientist and a wiser person. This thesis would not have been possible without her loving encouragements. 


\section{Contents}

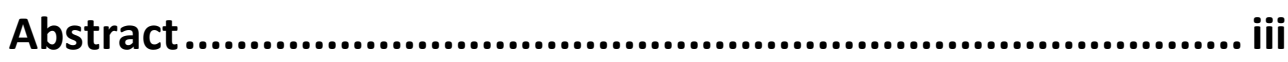

Acknowledgements ........................................................... vii

List of Figures ....................................................................

List of Tables ............................................................................

1. General Introduction ...................................................... 1

1.1 Marine populations, their spatial scale and structure ....................... 1

1.1.1 Connectivity of marine populations and the open-closed

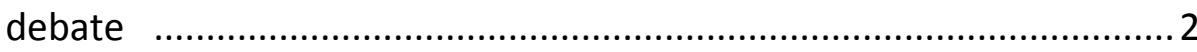

1.1.2 Connectivity and management of marine resources.............. 4

1.2 Studying larval dispersal in the marine environment ....................... 6

1.2.1 Simulating demographic connectivity................................ 6

1.2.2 Empirical measures of demographic connectivity: from

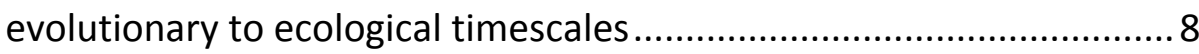

1.2.3 Otolith Chemistry as a marker of natal origins .................... 10

1.2.4 Profiles across otoliths: insights into dispersal histories ....... 13

1.3 Statistical methods for the analysis of otolith chemistry ................. 14

1.3.1 Bayesian methods for otolith chemistry............................. 17

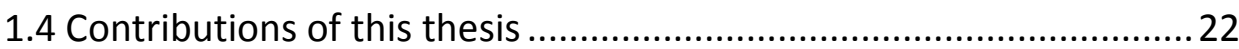

2. Scale-dependent variability in hatchling otolith chemistry: implications for studies of population * ................................ 27

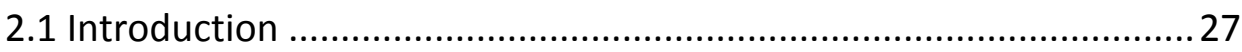

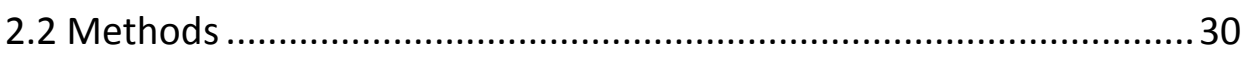

2.2.1 Study Species \& Sampling ............................................. 30

2.2.2 Otolith extraction, preparation and analysis ....................... 32

2.2.3 Statistical analysis ...................................................... 33 
2.3.1 Descriptive statistics, significance testing and discrimination...

2.3.2 Optimal grouping of sites by simulated annealing ............... 44

2.3.3 Exclusion test for groups of sites ..................................... 45

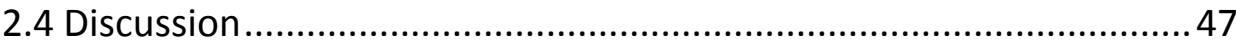

2.4.1 Scaling of variability in hatchling otolith signatures ............. 47

2.4.2 Optimal regional groupings by SA..................................... 49

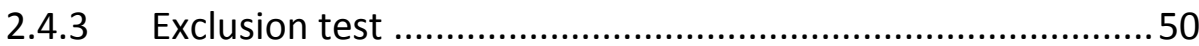

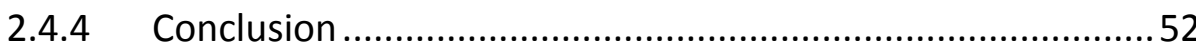

3. Characterizing natal sources of fish using flexible Bayesian mixture models for otolith geochemistry............................ 53

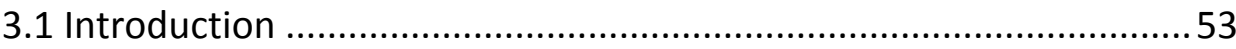

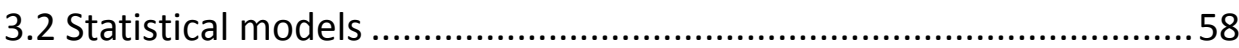

3.2.1 A Dirichlet process mixture (DPM) model for clustering .......58

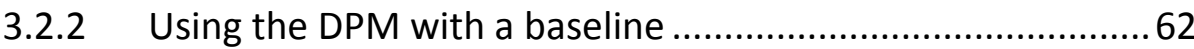

3.2.3 Extension to hierarchical classification with an incomplete

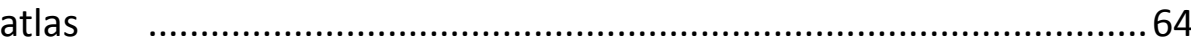

3.2.4 Marginal descriptions of source assignments.......................70

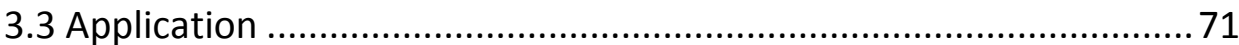

3.3.1 DPM clustering models ....................................................... 71

3.3.2 Clustering fish without a baseline ....................................... 72

3.3.3 Clustering fish with a baseline .......................................... 75

3.3.4 Classification with a (potentially) incomplete baseline ......... 80

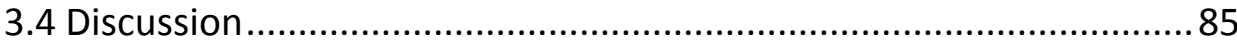

4. Larval dispersal pathways in a reef fish metapopulation: Contrasting empirical and simulation measures...................... 89 
4.2.1 Study system and otolith core chemistry atlas...................92

4.2.2 Recruit otolith preparation and pre-processing ................. 92

4.2.3 Selecting elements for analysis of otolith cores ..................95

4.2.4 Statistical analysis of otolith chemistry...............................96

4.2.5 Simulation models of dispersal ..........................................97

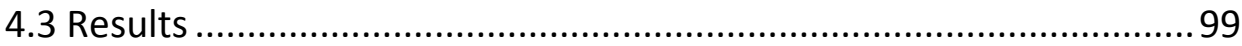

4.3.1 Results from otolith microchemistry .................................99

4.3.2 Results from hydrodynamic simulation experiments .......... 104

4.3.3 Comparing otolith and simulation results ............................... 111

4.4 Discussion

5. Plasticity and similarity in dispersal histories: a Bayesian framework for characterizing fish dispersal from otolith chemistry profiles .............................................................. 119

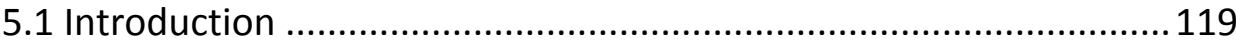

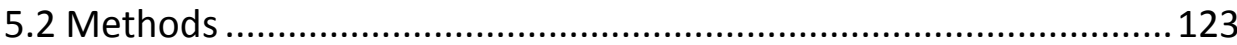

5.2.1 Generative models for chemical transects .......................123

5.2.2 The Bayesian approach: Prior knowledge and the lack thereof 128

5.2.3 Encoding alternate hypotheses ..................................... 129

5.2.4 Estimating the number of (distinguishable) environments along a transect 130

5.2.5 Temporal dynamics .......................................................... 132

5.2.6 Estimating unknowns: MCMC and WinBUGS .....................135

5.2.7 Characterizing contingents: a marginal visualization approach 
5.3.1 Simulation Experiment I: Increasingly complex simulation scenarios

5.3.2 Simulation Experiment II: Contingents from multivariate data.

5.3.3 Assessing models: posterior predictive checks and model selection

5.3.4 Application to Chinook salmon .......................................143

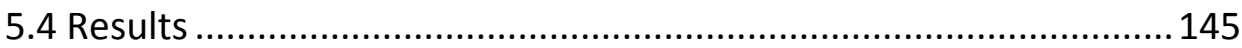

5.4.1 Simulation experiment I................................................ 145

5.4.2 Simulation experiment II .............................................. 148

5.4.3 Application to Chinook salmon ....................................... 150

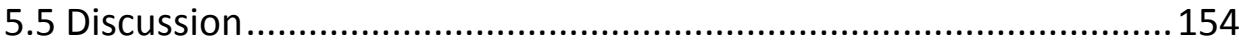

6. Inshore residency of fish larvae may maintain connections in a reef fish metapopulation .............................................. 161

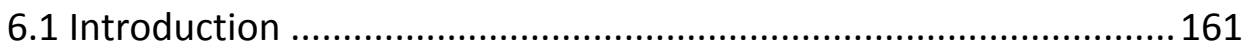

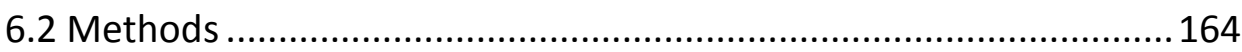

6.2.1 Recruit otolith preparation and pre - processing ............... 164

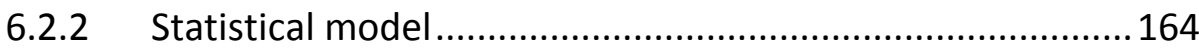

6.2.3 Hydrodynamic model investigation .................................... 168

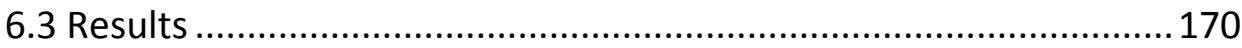

6.3.1 Results from models of dispersal histories ....................... 170

6.3.2 Hydrodynamic modelling results .................................... 173

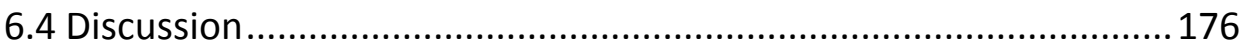

6.4.1 Mixture model results for dispersal histories ..................... 176

6.4.2 Interpreting dispersal patterns ....................................... 179

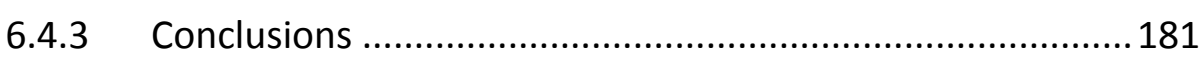


7. Discussion and perspectives ........................................ 183

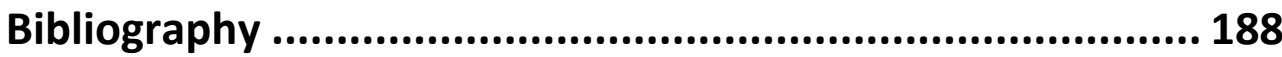

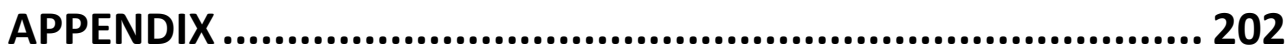

A.1 Weakfish exact linkage tree from the DPM clustering model........ 202

A.2 Gibbs sampling in the mixture models ........................................203

A.3 Drawing a state sequence from the HMM - the recursive method 204

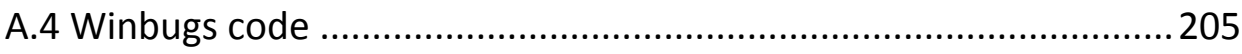




\section{List of Figures *}

Figure 1.1: Left to right, picture of the head of a larval common triplefin (Forsterygion lapillum) 12

Figure 1.2: Picture of an adult common triplefin (Forsterygion lapillum) ... 23

Figure 1.3: A Coastal boundary layer in Cook Strait .25

Figure 2.1: Map of New Zealand and the sampling domain 31

Figure 2.2: Kernel smoothed histograms of trace element signatures from the two main Islands along the linear discriminant function and regional differences along the first two linear discriminant axes.

Figure 2.3: Perspective plot showing predicted probability $(p)$ of correct assignment in a linear discriminant analysis in relation to number of groups and inter-group Mahalanobis distance.

Figure 2.4: Non-metric multi-dimensional scaling of sites within the total sampling domain

Figure 2.5: Kernel-smoothed histograms (black) of Mahalanobis distances to the centroid of the focal site 46

Figure 3.1: Illustration of the DPMc classification procedure (with a nonfixed baseline for the DPM)

Figure 3.2: Histogram of the marginal posterior distribution over the number of sources in the weakfish baseline dataset. .74

Figure 3.3: Exact linkage tree from a self-assignment DPM analysis with fixed baseline of weakfish juveniles. .76

Figure 3.4: Weakfish exact linkage tree from the DPM clustering model with fixed baseline .79 
Figure 3.5: Exact linkage trees from the DPMc classification procedure ....84

Figure 4.1 : Map of Cook Strait, New Zealand and the sampling domain ...93

Figure 4.2: Hatchling atlas element distributions using a Bayesian approach (Boxplot) 102

Figure 4.3: Assignment summaries for fish collected at the three reserve sites in Kapiti, Wellington and Long Island 103

Figure 4.4: Comparison of log transformed signatures from samples in the isolated cluster of Wellington sourced recruits vs. all other recruits assigned to Wellington 104

Figure 4.5: Tracers concentration $12 \mathrm{~h}, 48 \mathrm{~h}, 7$ days and 14 days after being released at sites around Kapiti Island 106

Figure 4.6: Tracers concentration $12 \mathrm{~h}, 48 \mathrm{~h}, 7$ days and 14 days after being released at sites on Wellington's south coast. 107

Figure 4.7: Tracers concentration 12h, $48 \mathrm{~h}, 7$ days and 14 days after being released at sites in Wellington's harbour. 108

Figure 4.8: Tracers concentration $12 \mathrm{~h}, 48 \mathrm{~h}, 7$ days and 14 days after being released at sites in the Marlborough Sounds. 109

Figure 4.9: Mean current velocities in Cook Strait predicted by the Gerris flow solver ocean model 110

Figure 4.10: Dispersal pathways inferred by otolith chemistry (arrows) .. 112

Figure 5.1: Example profiles for Strontium and Barium across Chinook salmon otoliths 124

Figure 5.2: Directed acyclic graphs of the simple mixture model for chemical transects across otoliths and the random effects mixture model 
Figure 5.3: A hidden Markov model with individual transition matrices $\pi$ for each fish i

Figure 5.4: The $3 \times 3$ grid of (hypothetical and arbitrary) near-shore to offshore variations in elemental concentration 139

Figure 5.5: True and estimated partitions for a random effects mixture model, a hidden Markov model (HMM) with a common transition matrix and a HMM with individual matrices for each individual fish 148

Figure 5.6: True and estimated partitions for a Dirichlet process random effects mixture model, a mixture model with a single mixture proportion over all fish and a hidden Markov model with a single transition matrix .149

Figure 5.7: Marginal posterior distribution over the number of contributing near-shore sources in simulation experiment II. 149

Figure 5.8: Visualization of marginal similarity of transects from simulation experiment II 151

Figure 5.9: Prior (dotted) versus posterior (line) distributions of water-mass means of Strontium ( $\mathrm{Sr}$ ) and Barium for the three component model for Chinook salmon 152

Figure 5.10: Histograms of the marginal posterior distribution over per cent emigrating size classes and posterior distribution of size of Central California Valley Chinook salmon at freshwater emigration. 154

Figure 6.1: Example of three ${ }^{138} \mathrm{Ba}: \mathrm{Ca}$ traces and resulting density estimates 166

Figure 6.2: 6 hourly average wind direction and wind speed (in knots (kn)) at Wellington airport 170

Figure 6.3: Modal estimated dispersal patterns of all fish at each site, sorted by number of frames with inshore signatures 174 
Figure 6.4: Visualization of Gerris simulation results of flow velocity....... 175

Note that figure captions are abbreviated and/or slightly modified from those in the text 


\section{List of Tables *}

Table 2.1: Distribution of trace element concentrations relative to

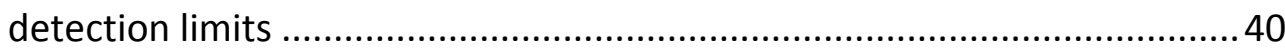

Table 2.2: Variance explained and $P$ values from nested permutational MANOVA

Table 2.3: Exclusion test results in per cent of hatchlings correctly excluded from the focal location using full and truncated datasets .45

Table 3.1: Posterior expectation and mode for the number of sources in each of four simulation experiment. .73

Table 3.2: Results from the assignment test 78

Table 3.3: Comparison of the proportion $\mathrm{P}_{\mathrm{c}}$ of correctly assigned individuals in a self-assignment test for the weakfish and triplefin datasets 82

Table 4.1: Distribution of trace element concentrations relative to detection limits 101

Table 5.1: Simulation parameters for experiments I and II 140

Table 5.2: Prior parameters used for the Chinook salmon dataset 145

Table 5.3: Simulation results for each simulated scenario 147

Table 6.1: Expectation and 95 per cent credible interval of the posterior distribution of some parameters in the dispersal model 171

Table 6.2: Pairwise $p$-values from randomization tests 173

* Note that table captions are abbreviated and/or slightly modified from those in the text 


\section{Chapter 1:}

\section{General Introduction}

\subsection{Marine populations, their spatial scale and structure}

The concept of a "population" is essential to the discipline of ecology, yet its precise definition has been much debated (Wells \& Richmond 1995, Berryman 2002, Camus \& Lima 2002, Schaefer 2006). Despite the disagreement on the exact definition of the population, most authors agree that the utility of the population concept, as linked to the ability to study dynamics of units of a species with high predictive power, is highest at a scale where these dynamics are dominated by birth and death processes (Camus \& Lima 2002, Schaefer 2006).

Levins (1969) introduced the metapopulation model to study the effect of fragmentation and dispersal on the persistence of the "metapopulation" as defined by connected local populations or patches. The metapopulation is defined as the scale at which it is (at least on ecological timescales) isolated through dispersal boundaries from other metapopulations, and its dynamics are globally dominated by birth and death processes. The metapopulation in this sense is equivalent to the population concept (sensu Camus and Lima (2002)), and differs only in that it explicitly considers fragmentation and connectivity within the population. Local population or patch dynamics within the metapopulation are influenced by dispersal, often in the form of dispersal of propagules (i.e. larvae or seeds) 


\subsection{Marine populations, their spatial scale and structure}

or adult movement (Armsworth 2002, Smedbol \& Wroblewski 2002, Kritzer \& Sale 2004).

The metapopulation concept has been widely applied in terrestrial ecology to address a number of questions related to the influence of spatial structure of populations on overall metapopulation persistence and dynamics. Indeed, Levins' (1969) original paper was concerned with population persistence on evolutionary timescales, which can be shown to be dependent on the ratio of extinction- versus re-colonisation rates of patches. Hence, the degree of connectivity between patches plays a key role for metapopulation dynamics. The concept has since evolved to include dynamics other than persistence (see Hanski 1999 for a review), yet its utility for the study of marine populations has been debated (Smedbol \& Wroblewski 2002, Kritzer \& Sale 2004). The prevalence of spatially structured populations that are potentially connected by dispersal of adults or larvae make this framework a natural model for population dynamics in the ocean and especially for inshore (i.e. estuarine) and reef associated species (Sale 1998, Thorrold et al. 2001, Armsworth 2002, Hastings \& Botsford 2006, White et al. 2010).

\subsubsection{Connectivity of marine populations}

The spatial arrangement and resulting connectivity of local populations of a species play a central role in ecological and evolutionary processes (Levins 1969, Jax et al. 1998, Armsworth 2002, Hastings \& Botsford 2006), with consequences for conservation planning and resource management (Roberts 1998, Botsford et al. 2001, Lockwood \& Hastings 2002). Connectivity amongst patches and populations has been notoriously difficult to assess in the marine environment (Sale et al. 2005, Halpern et al. 2006). Most marine fishes and invertebrates possess a pelagic larval phase, which potentially allows for long distance dispersal via ocean currents and large scale mixing of genetic material (Palumbi 2003). The concealed nature of the marine environment, its multi-scale environmental 
stochasticity and high larval mortality rates make the tracing of larval dispersal a considerable challenge.

Consequently, ecologists in the second half of the $20^{\text {th }}$ century studied dynamics of marine species from the perspective of "open populations", in what is often referred to as the "supply side" ecology paradigm (Levin 2006). Indeed, early studies linking population dynamics to larval supply found that species with protracted larval stages exhibited larger fluctuations than those without. This phenomenon was formulated in the recruitment limitation hypothesis, which states that marine population dynamics are largely determined by fluctuations in larval supply (Doherty 1981, Caley et al. 1996), and that subsequent (density dependent) processes during settlement are of limited relative importance. Furthermore, an "open" population would rely on externally supplied larvae for recruits, whereas a "closed" population could sustain itself through retention and self-recruitment. Marine populations were generally seen as being open on scales relevant to studies (and thus recruitment limited to some extent), making them fundamentally different from stocks in fisheries science or metapopulations in terrestrial systems, which are closed populations by definition (Caley et al. 1996).

This distinction is, however, a matter of scale (Caley et al. 1996, Armsworth 2002, Camus \& Lima 2002, Kinlan et al. 2005), and this scale is likely to be species specific (Kinlan et al. 2005). The paradigm of open populations in the marine environment as well as the supply side focus can thus be seen as a consequence of limited knowledge of larval dispersal coupled to an operational definition of the population concept itself: with limited to no knowledge of dispersal patterns between local marine populations, one cannot delimit a (meta-)population. In recent years however, numerous studies have questioned the validity of the concept of open populations for marine organisms (Jones et al. 1999, Swearer et al. 1999, Cowen et al. 2000, Thorrold et al. 2001, Swearer et al. 2002, Eckert 2003). Further insight into mechanisms of larval dispersal (Leis \& Stobutzki 


\subsection{Marine populations, their spatial scale and structure}

1999, Paris \& Cowen 2004, Lecchini et al. 2005, Siegel et al. 2008) shifted focus from the paradigm of open populations to the actual scales of connectivity and population processes in marine populations (Cowen et al. 2000, Cowen et al. 2006, Treml et al. 2008). Nevertheless, demographic connectivity in marine metapopulations has remained enigmatic; especially the empirical tracking of dispersing larvae remains a daunting task for marine scientists.

\subsubsection{Connectivity and management of marine resources}

The sustainability of marine resources is a major concern as large parts of the world's population are dependent on marine resources (Botsford et al. 1997, Pauly et al. 2002). In fact, resource management and conservation issues are nowadays linked for the majority of marine systems around the world, as historical (over)fishing and resource exploitation have affected most ecosystems (Jackson et al. 2001, Pauly et al. 2005, Halpern et al. 2008). Consequently, classical conservation tools such as reserves have been invoked as possible tools for resource management (Roberts et al. 2001, Avasthi 2005, White \& Kendall 2007). Such management and protection measures have traditionally been performed at the scale of single species stocks and populations (Pauly et al. 2002), although ecosystem based management is often seen as a more viable alternative to single species management (Pikitch et al. 2004, Arkema et al. 2006).

Management of populations for sustainable exploitation or conservation relies on conceptual and/or mathematical models of population dynamics, which in turn rely implicitly or explicitly on assumptions of patch connectivity within a species range and our ability to delimit populations within a species' range. Fisheries, for example, are often subdivided into stocks (or fishing zones) with attached quotas and management plans, implicitly assuming independence of stocks (within species between zones and between species in a single zone) (i.e. The New Zealand Quota Management System which encompasses a number of Fisheries Management Areas; (Kelly \& Stefan 2007)). Similarly, catch 
statistics are usually compiled for each "fishery" and used as model input. Such models include necessary parameters or assumptions of population (or stock) structure and the degree of dependence of subpopulations (Schnute \& Richards 2001). Due to the often hidden nature of population processes in the sea, few of these parameters are readily measured. They usually contain considerable uncertainty, and can cause considerable differences in outcome of model scenarios (Clark 1996, Schnute \& Richards 2001, Halpern et al. 2006).

The uncertainty involved in traditional fisheries management and stock modelling has prompted numerous fisheries scientists to suggest marine reserves as ecosystem based management tools (Clark 1996, Bohnsack 1998, Roberts et al. 2001, Pauly et al. 2002). This idea goes back to Beverton \& Holt (1957), but was then thought to be suboptimal when compared to other management alternatives (Guenette et al. 1998). The collapse of numerous fish stocks worldwide initiated a renewed interest in this idea, and initial concerns about potential decrease in yield for this management strategy (Hastings \& Botsford 1999) have since been contradicted (Botsford 2005, White \& Kendall 2007).

The efficacy of marine reserves again hinges on the concept of connectivity between reserves as well as reserves and fished areas (Crowder et al. 2000, Shanks et al. 2003, Halpern et al. 2006). For a (sub-) population inside a reserve or reserve network to be viable, it needs to be either self-sustaining (closed) or receiving propagules from upstream patches. As such, the persistence of a species within a reserve (network) largely depends on connectivity patterns among patches (Botsford et al. 2001, Lockwood \& Hastings 2002, Shanks et al. 2003, Halpern et al. 2006, Hastings \& Botsford 2006).

Two potentially beneficial effects of marine reserves to adjacent fisheries are spillover of adults as they emigrate from the reserve and recruitment subsidies as larvae from protected spawning stocks are 


\subsection{Studying larval dispersal in the marine environment}

exported across reserve boundaries. For the latter process to be an effective enhancement of fished populations, the connectivity between protected and fished stocks needs to be known before reliable estimates of benefits beyond protection can be made (Stobutzki 2001). Few reliable estimates of connectivity amongst reserves or recruitment subsidies to fished areas are available today (but see Almany et al. 2007, Cudney-Bueno et al. 2009, Planes et al. 2009) and finding appropriate ways to assess such connectivity remains a challenge to the effective use of reserves as management tools (Sale et al. 2005).

\subsection{Studying larval dispersal in the marine environment}

\subsubsection{Simulating demographic connectivity}

The importance of dispersal in determining dynamics of spatially structured populations has led to a surge of simulation modelling efforts in this domain. Oceanographic models of ocean currents, often coupled with larval behaviour and ecology, have allowed important insights into dispersal scenarios under various conditions (Gilg \& Hilbish 2003, Paris \& Cowen 2004, Cowen et al. 2006, Siegel et al. 2008).

Individual-based modelling of early life histories of exploited marine populations emerged in the 1980s and has developed quickly to become a widely used tool in fisheries science (Miller 2007b). Fluctuations of year classes of fished stocks remain the biggest problem to fisheries management (Hutchings 2000), and modelling efforts to link year class variability to early life history biology have led to ever more complex biophysical models of larval dispersal for fish stocks (reviewed in Marine Ecology Progress Series 2007:347 Theme section- Advances in modelling physical-biological interactions in fish early life history). Recent developments in this field stress the importance of behaviour and trophic interactions on various scales for the outcome of the dispersal process (Fiksen et al. 2007, Leis 2007). Larvae are known to position themselves 
vertically with respect to flow fields and horizontally with respect to settlement cues (Leis \& Stobutzki 1999, Leis \& Carson-Ewart 2003, Lecchini et al. 2005). This behaviour may greatly influence dispersal distance, trajectories and retention rates of larvae near spawning sites (Paris \& Cowen 2004, Fisher 2005). Furthermore, the food availability for dispersing larvae may significantly alter growth and mortality of larvae and thus determine dispersal outcomes (the "match-mismatch hypothesis" (Cushing 1990)), requiring inclusion of lower trophic levels (Peck \& Daewel 2007). The complexity of the dispersal process becomes ever more apparent in the light of these modelling studies (see also Siegel et al. 2008).

Most models in more theoretically oriented studies use advectiondiffusion equations and Lagrangian particle descriptions as virtual larvae in order to develop connectivity matrices as dispersal probabilities between any two sites (Largier 2003, Siegel et al. 2003). Recent results indicate that connectivity measures on short timescales bear considerable stochasticity (Mitarai et al. 2008, Siegel et al. 2008), resulting from the chaotic nature of ocean currents under variable forcing. Thus, larval dispersal routes may be inherently unpredictable on small spatial- and short timescales, with largely heterogeneous settlement depending on a number of parameters such as number of released larvae, their biological parameters, as well as physical parameters of the ocean. The interplay of these parameters, producing a highly stochastic outcome, highlights the difficulty in predicting larval dispersal from such models for ecologically relevant timescales.

Early modelling efforts use larvae as passive particles in a flow field (Roberts 1997), describing potential larval routes under different pelagic larval durations, supporting the paradigm of high connectivity in marine populations. Incorporation of biological parameters such as mortality and swimming behaviour may lead to drastically different scenarios of connectivity in the same region (Cowen et al. 2000, Paris \& Cowen 2004, Cowen et al. 2006), further underlining the dependence of model outcomes on biological input parameters. These results indicate that, 


\subsection{Studying larval dispersal in the marine environment}

despite long larval durations, long distance dispersal may be rare rather than being the norm. Mortality may act to reduce inter-(major) island dispersal probabilities in the Caribbean to being close to zero (Cowen et al. 2000), while swimming behaviour may act as an active mechanism by which larvae can maintain themselves in favourable flows for remaining near shore in a vertically stratified current field (Paris \& Cowen 2004, Fisher 2005, Leis 2007), thus limiting advection into the open ocean. Similar modelling of connectivity on the Great Barrier Reef, including constant larval mortality, highlights the importance of self-sustaining patches in the metapopulation (James et al. 2002). Nevertheless, these simulations also indicate that considerable larval exchange between patches is possible under the model, possibly due to the less isolated nature of the Great Barrier Reef as opposed to the Caribbean modelled by Cowen and collaborators (2000).

Taken together, these works show that deterministic modelling of larval pathways may lead to heterogeneous dispersal kernels, which change and become increasingly stochastic with increasing model input (see also White et al. 2010). Thus, these dispersal estimates may be most useful as hypothesis-generating tools as well as for understanding dispersal as long-term averages of these stochastic connectivity matrices (Siegel et al. 2008). Such hypotheses can then guide empirical studies into realized dispersal patterns and mechanisms (Gilg \& Hilbish 2003), and this approach is used for two of the chapters in this thesis (see chapters $4 \& 6$ ).

1.2.2 Empirical measures of demographic connectivity: from evolutionary to ecological timescales

Instead of asking the question of where larvae go, as is mostly done in simulation models of dispersal, the question of where settlers and recruits came from may be more readily answered and may thus give important clues about different parameters involved in the dispersal process (Levin 2006). 
Population genetics have a long history of assessing relatedness of populations of species in the form of gene flow between populations. Gene flow, together with selection, mutation and drift, is one of the four driving forces in evolution, and thus the rate of gene flow between populations may determine the rate of differentiation of populations and ultimately speciation. It can be shown that even a low number of migrants (relative to mutation, selection and drift) between populations can maintain homogenous allele frequencies between populations (panmixia), whereas the absence of dispersers will lead to diverging allele frequencies (Palumbi 1994).

These considerations have made population genetics a natural choice for investigation of demographic connectivity in the marine environment (reviewed in (Hellberg et al. 2002, Palumbi 2003, Hellberg 2007). The timescales over which connectivity can be resolved depend on the variability of the marker used. Conserved markers, such as allozymes and mtDNA, are useful on vast geographic and temporal scales, for example to infer evolutionary events related to a species distribution (phylogeography). These markers integrate dispersal over many (100s1000s) generations and are thus rarely suited to infer dispersal on ecologically relevant timescales (but see Taylor \& Hellberg 2003). More variable markers such as microsatellites and AFLP fingerprinting have the power to detect differentiation over ecologically relevant timescales, to as low as 20 generations (Hellberg 2007). Paired with multi-locus assignment techniques these markers can be used to infer dispersal events and contemporary migration (e.g. Baums et al. 2006, Shank \& Halanych 2007, Bradbury et al. 2008), yet the limitations of these techniques are not yet known in the marine environment as applications are scarce thus far (Hauser et al. 2006, Waples \& Gaggiotti 2006, but see Ruzzante et al. 2006, Planes et al. 2009).

Environmental markers have recently emerged as a promising tool for assessing connectivity in single generations by assigning recruits to source 


\subsection{Studying larval dispersal in the marine environment}

populations based on their natal "fingerprint" (Campana \& Thorrold 2001, Thorrold et al. 2002). Such markers may be natural or artificial, yet artificial markers are often seen as being of limited use due to extremely high mortality rates for dispersing larvae, making recapture of marked larvae unlikely. Some successful applications of artificial marking exist (Jones et al. 1999, Almany et al. 2007), revealing patterns of retention and selfrecruitment in island populations. Trans-generational marking of larvae by injection of markers into spawning females is a promising new tool allowing for marking of several thousand eggs (Thorrold et al. 2006), yet the logistic problem of having to examine extremely high numbers of recruits for recapture probably remains.

Natural fingerprints are markers which record the environmental conditions of the organism, and are as such present in every individual of the species. Natural tags can be found in the trace element and isotopic composition of otoliths (fish), prodissoconch (bivalves) or statoliths (molluscs) (Levin 2006). Such natural markers have been employed in a variety of settings to date, from reconstruction of migratory pathways (Elsdon \& Gillanders 2003, Campana et al. 2007) to identification of natal (Thorrold et al. 2001, Becker et al. 2007) and nursery habitats (Gillanders \& Kingsford 2000, Forrester \& Swearer 2002) as well as dispersal histories (Swearer et al. 1999, Hamilton et al. 2008, Shima \& Swearer 2009a, 2010). Recent work combining contemporary genetic dispersal estimates with otolith microchemistry illustrates how these two techniques can give complimentary and mechanistic information on dispersal and population structure (Feyrer et al. 2007, Bradbury et al. 2008).

\subsubsection{Otolith Chemistry as a marker of natal origins}

In teleost fishes, otoliths are known to act as environmental recorders during the fish's life, with aragonite type calcium carbonate depositions forming daily growth rings (Campana \& Thorrold 2001, Campana 2005). Divalent ions, such as $\mathrm{Sr}, \mathrm{Mg}, \mathrm{Ba}$ and $\mathrm{Mn}$ ions, may substitute for $\mathrm{Ca}$ ions during deposition (Campana 1999, Brophy et al. 2004) (but their affinity for 
aragonite may vary; their inclusion rates are not correlated; (Campana 1999)). Although the physiological barriers to inclusion of such elements may be variable from one element to the other, trace elements, which are not usually osmoregulated, may be included into the otolith at rates reflecting environmental conditions. Once deposited, these records are permanent, owing to the fact that otoliths are metabolically inert.

Concentrations of ions such as $\mathrm{Sr}$ and $\mathrm{Ba}$ are known to be affected by temperature, salinity and environmental concentrations in adult otoliths (Campana 1999, Elsdon \& Gillanders 2004, Elsdon \& Gillanders 2005). Other elements are thought to be associated with anthropogenic activity: $\mathrm{Pb}$ and $\mathrm{Br}$ for example are usually enriched in coastal waters. Taken together, these elemental fingerprints may give information about environmental conditions experienced by a fish and may thus allow reconstruction of dispersal histories back to the source population (Ruttenberg et al. 2005).

For this approach to be useful, a number of conditions should be met: i) otolith chemistry should be sufficiently variable over the spatial extent of possible dispersal pathways in the species of interest. Ideally, this variability would reflect signatures of individual sub-populations in the metapopulation, allowing for estimation of connectivity between any two sub-populations. ii) To avoid having to sample from matching cohorts, signatures should be temporally stable or directly correlated to environmental conditions, which could consequently serve as proxies for otolith chemistry. iii) Possible source populations should be known and sampled extensively. 


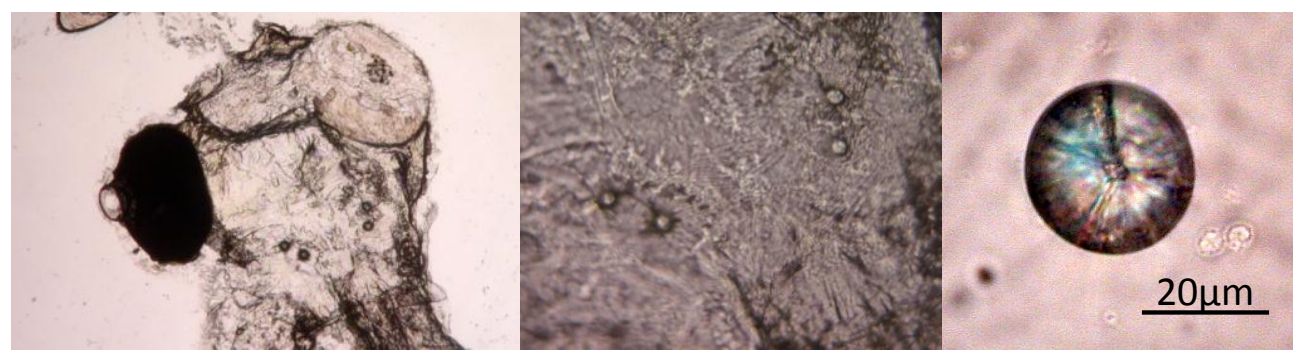

Figure 1.1: Left to right, picture of the head of a larval common triplefin (Forsterygion lapillum) immediately after hatching showing larval otoliths in transparency, zoom on otoliths and a close-up of an extracted larval otolith. This hatchling portion of the otolith is the basis for the analysis of the natal chemical signature contained within the otolith core - the natal region of an otolith.

Recent studies in fishes such as anadromous galaxids (Barbee \& Swearer 2007), cyprinids (Feyrer et al. 2007), diadromous salmon (BarnettJohnson et al. 2008), estuarine spawning weakfish (Thorrold et al. 2001), snapper (Gillanders 2002, Gillanders 2005) and smelt (Bradbury et al. 2008) indicate that in systems with sufficiently different water chemistry, natal habitats may be distinguished with relatively high confidence. However, all of these studies investigate estuarine or river systems, which often vary considerably over relatively small spatial scales (Bradbury et al. 2008).

Warner and colleagues (2005) investigated natal signatures in the otolith core (see also Figure 1.1) of rockfish along the Californian coast in relation to water chemistry and diffusive gel (DGT) measurements. These authors found significant between-site variation for most elements as well as for multivariate measures, though variation was dependent on the year of collection, indicating temporal instability of signatures. Furthermore, neither water chemistry nor DGT measures seemed to correlate consistently with otolith signatures. Ruttenberg \& Warner (2006) and Ruttenberg et al. (2008) examined the spatial scales of variation of natal signatures over a range of geographical scales. Both studies found useful between-site variation comparable to the study of Warner et al. (2005), but no significant variation between regions. Thus, variability may be most significant on smaller scales than actual population scales. Not much is 
known about the deterministic nature of these scales, and maternal effects (Brophy et al. 2004, Chittaro et al. 2006) and small-scale changes in concentration of elements in the water column (Campana 1999) may influence otolith microchemistry on small scales and thus make signatures too variable to be useful on larger scales. Thus, before this method can be successfully applied to connectivity problems, the geographical resolution and scale of variation of natal otolith signatures needs to be established. This basic need provides the motivation for the first chapter of this thesis.

\subsubsection{Profiles across otoliths: insights into dispersal histories}

While otolith cores and the surrounding region may lead to insights about natal origins of fish, profiles across otoliths of fish can provide considerable information about their dispersal (Elsdon et al. 2008). Due to the concentric growth of otoliths, time resolved measurements along the growth axis may for instance provide information about the series of environments a fish visited over the measured time (Elsdon et al. 2008), and thereby give insights into ontogenetic shifts in habitat use (Miller et al. 2010), larval dispersal histories (Shima \& Swearer 2009a, Shima \& Swearer 2009b, Shima \& Swearer 2010) and their demographic consequences as well as adult migration patterns (Fowler et al. 2005). Studies of dispersal pathways have further contributed to the definition of migratory contingents within the concept of fish stocks (Secor \& Piccoli 1996, Secor 1999). Contingents are intra-population groups of fish which display similar migration histories, the existence of which can have important implications for demographic processes and management (Secor 1999, Miller et al. 2010).

In order to reconstruct dispersal pathways from profiles across otoliths, one needs to have an idea about the scales of variability in otolith signatures in a system, and be able to connect a given signature to a unique water mass. Again, this can be straightforward for diadromous fish, but it may be more difficult in marine environments, where chemical gradients are usually smoother and scales of variation are not immediately 


\subsection{Statistical methods for the analysis of otolith chemistry}

obvious. The analysis of otolith profiles is ideally paired with studies of variability within water masses (Elsdon et al. 2008). Often however, hypotheses can be generated about the effects of different water masses on otolith chemistry due to known relationships. In these situations, the otolith profiles may be analysed in the light of such assumptions (Hamilton et al. 2008, Shima \& Swearer 2009a).

\subsection{Statistical methods for the analysis of otolith chemistry}

Whether it is isolated regions of the otolith or time resolved profiles, the statistical analysis of otoliths is an important step in drawing robust conclusions from this type of data. Data obtained from chemical analyses of otoliths (usually using Laser ablation inductively coupled plasma mass spectrometry) is noisy for a number of reasons, including environmental variability (auto-correlated noise), measurement noise due to contaminations (auto-correlated noise if contaminations are strong enough) and measurement noise due to the method itself (white or Gaussian noise). While the latter type of noise is usually not much of a problem, the former two forms of noise can significantly alter the inferences we make from otolith chemical measurements. Robust processing and statistical analysis of such data is thus of foremost importance.

Most recent analyses of otolith chemistry rely on either mixture models or linear discriminant analysis for the characterization and discrimination of water-masses from isolated scans of otolith chemistry (White \& Ruttenberg 2006). These models are intimately related, in that the discriminant function analysis is a sub-class of a mixture model where a single parameter is fixed. The joint probability density for a mixture model can be written as: 


$$
f(\boldsymbol{y} \mid \pi, \theta)=\prod_{i=1}^{N} \sum_{k=1}^{K} \pi_{k} f\left(y_{i} \mid \theta_{k}\right)
$$

The formalism in equation (1.1) states that the data $\boldsymbol{y}=\left(y_{1} \ldots y_{i} \ldots y_{N}\right)$ are distributed as a mixture density, with proportions $\pi=\left(\pi_{1} \ldots \pi_{k} \ldots \pi_{K}\right)$ originating from one of $K$ water-masses. $f\left(y_{i} \mid \theta_{k}\right)$ is the conditional density of water mass $k$, parameterized by $\theta_{k}$ (with $\theta=\left(\theta_{1} \ldots \theta_{k} \ldots \theta_{K}\right)$ ) evaluated at $y_{i}$ (the likelihood). One usually chooses a normal (or Gaussian) density for $f$, although other distributions could be used. The proportions of fish from water-mass or source $k$ is then $\pi_{k}=p\left(\xi_{i}=k\right)$, where $\xi_{i}$ assigns individual $i$ to source $k$. From this formulation it is possible to obtain the posterior distribution of a fish belonging to one of $K$ water masses via Bayes theorem:

$$
p\left(\xi_{i}=k \mid \theta, \boldsymbol{y}\right)=\frac{\pi_{k} f\left(y_{i} \mid \theta_{k}\right)}{\sum_{k^{\prime}=1}^{K} \pi_{k^{\prime}} f\left(y_{i} \mid \theta_{k^{\prime}}\right)}
$$

The only difference between a mixture model and a discriminant analysis is that for the latter the $\pi_{k}$ are assumed to be known. Often, homogeneity of variances is assumed as well (in linear discriminant analysis for instance). If $f(\boldsymbol{y} \mid \theta)$ is known, say from a baseline sample, then assigning fish to a water-mass or source can be directly done using equation (1.2) by assigning to the source with the highest associated posterior probability. If one treats the mixing proportions $\pi_{k}$ as unknown, these need to be estimated via maximum likelihood or Bayesian methods. With Bayesian methods, $f$ may be treated as unknown as well and estimated from the baseline sample at the same time (Munch \& Clarke 2008, White et al. 2008).

Other methods can be used to assign fish to natal sources or different water-masses. Thorrold et al. (1998) for instance used artificial neural networks for classification of individual fish. While they found this technique relatively successful, neural networks are prone to over-fitting and this study remains the only study to have used this formalism in an 


\subsection{Statistical methods for the analysis of otolith chemistry}

empirical context. Mercier et al. (in press) evaluated the performance of linear discriminant analysis against machine learning techniques including random forests and artificial neural networks. These authors found that machine learning methods were more robust when the assumptions of discriminant analysis were not met. A possible drawback of these methods however is that they rely on a single baseline sample, and that baseline characteristics are thus assumed to be known. A common limitation to all of the above models is that the potential sources are assumed to be known. This can be especially problematic in the marine environment where it may not be possible to characterise all possible natal regions. This limitation will be discussed in the following section.

The array of techniques used for the statistical analysis of otolith profiles is larger still, reflecting the diversity of questions that can be addressed with this type of data. The time-resolved nature of the data necessitates special attention since measurements along the otolith profile are usually not independent (Elsdon et al. 2008). Methods such as repeated measures ANOVA, its mixed model analogue, and Multivariate ANOVA (MANOVA) have been proposed to assess statistical significant differences between sets of fish and their individual time-resolved signatures (Secor \& Piccoli 1996, Elsdon et al. 2008).

To answer more intricate questions, Shima and Swearer (2009a) proposed a clustering framework based on time-series descriptors to discover similarities in dispersal histories. Sandin et al. (2005) proposed a likelihood framework to classify fish to ad hoc migratory classes based on otolith profiles, and Hamilton et al. (2008) used an extension of this framework to assign fish to retained or dispersed phenotypes. Finally, Fablet et al. (2007) used a latent state modelling approach, comparing mixture models with a hidden Markov model, to study habitat transitions of European eels. This approach can be seen as a time-dependent extension of the above mentioned mixture model (c.f. Chapter 4). 


\subsubsection{Bayesian methods for otolith chemistry}

Bayesian methods have dominated recent publications on mixture modelling from both geochemical and genetic markers (Pella \& Masuda 2001, Manel et al. 2005, Pella \& Masuda 2006, Bolker et al. 2007, Munch \& Clarke 2008, White et al. 2008, Smith \& Campana 2010). Of these, three methods were specifically concerned with a Bayesian analysis of otolith chemical signatures (Munch \& Clarke 2008, White et al. 2008, Smith \& Campana 2010). While Smith \& Campana's model is designed to accommodate genetic data also, the distinction between the type of data is actually of little relevance to the model itself - the underlying sampling distribution simply changes from a normal distribution for otolith data to a multinomial one for genetic data and their likelihoods are multiplied for each fish. The basic model setup is the same as in Bolker et al. (2007) for genetic data, which in turn is adapted from Pella \& Masuda (2001). Thus, the distinction of methods for otoliths versus genetic data relies simply on the sampling distribution used; the underlying models however are equivalent.

Within these models (and other models discussed above) we may distinguish conditional and unconditional models. Conditional models receive their name because estimates of quantities of interest, such as assignment probabilities and mixing proportions, are conditional on a baseline sample and it's estimated parameters. Thus, once these characteristics are determined, they are considered to be fixed. This is the setting of classical classification methods under which earlier mixture models (Millar 1987) discriminant anlysis, neural networks, so-called random forrests and related methods employed for classification based on otolith chemistry can be grouped. Unconditional models on the other hand will update the baseline in the light of the mixed sample at hand: the parameters $\theta$ are shared between the mixed sample and the baseline. Koljonen et al. (2005) found that these methods can perform significantly better than conditional methods, especially if baseline sizes are small 


\subsection{Statistical methods for the analysis of otolith chemistry}

compared to the mixed stock. This is often the case in fisheries, rarely however in ecological studies.

What differentiates all of the models mentioned in the first paragraph of this section from earlier models for mixed samples of unknown origin is their use of Bayesian inference. Bayesian inference makes use of Bayes theorem to draw inference about quantities of interest, such as model parameters. Bayes theorem is expressed as:

$$
p(M \mid D)=\frac{p(D \mid M) p(M)}{p(D)}
$$

Where the expression $p(A \mid B)$ indicates the conditional density of $A$ given B. It states that, given a set of data, the posterior beliefs in a model $M$, characterized by a set of parameters, is determined by the information received from the data $D$ (conveyed by the likelihood $p(D \mid M)$ ), multiplied by our prior beliefs in the model, divided by the total probability of the data (which is independent of the model and therefore sometimes written as a constant) (Gelman et al. 2003). To compute our posterior belief in the model, we thus need to be able to i) specify a distribution which reflects our prior beliefs about model parameters, ii) express the joint likelihood of the model parameters, and iii) calculate the constant in the denominator. Often it is the last of these three points that poses a problem: it involves an integral over all unknown model parameters, such that $p(D)=\int p(D \mid M) p(M) d M$ becomes a multiple integral which is most often not analytically solvable. Sampling based methods can however be used to draw samples from the posterior distribution of conditional quantities, for instance via Markov Chain Monte Carlo (MCMC) algorithms.

Consider for instance the conditional probability of parameter $\theta_{i}$, given the remaining parameters in the model and the data at hand. This can be expressed as $p\left(\theta_{i} \mid \theta^{-i}, D\right)$, where the superscript ${ }^{-i}$ indicates the set of parameters $\theta$ except individual parameter $\theta_{i}$. Iteratively drawing from these conditional distributions is referred to as Gibbs sampling (Gilks et al. 
1996), a method which will asymptotically recovery the conditional posterior distribution for each parameter. A multitude of other variants of MCMC exist for situations in which we cannot explicitly write such conditional distributions. Though different in terms of their efficiency, all of these methods guarantee that samples will eventually be from the actual posterior distribution. It is however not guaranteed that this happens in any given amount of draws from the sampling scheme. It is therefore important to either devise efficient algorithms which quickly converge to the correct distribution, or run the sampler for a great number of iterations (no method can however guarantee that this distribution has actually been reached or fully explored by the resulting draws - which is usually not much of a problem in practice when efficient schemes are used).

Illustration: Estimating parameters in the mixture model - Directed Acyclic Graphs (DAG) and Gibbs sampling

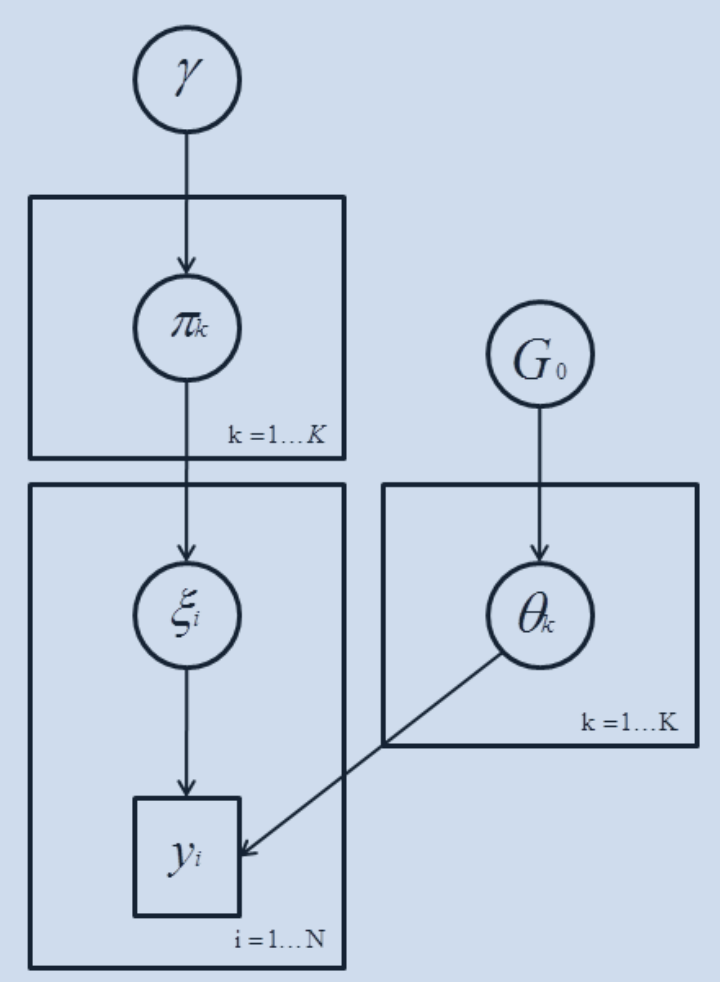

To illustrate how Bayesian models can be used to estimate parameters in the mixture model in equation (1) I first introduce directed acyclic models, a sub-form of graphical models (Jordan 2004). Graphical models (also called a Bayesian networks) depict conditional independence relationships of random variables in a model. Consider for instance 


\subsection{Statistical methods for the analysis of otolith chemistry}

the mixture model considered in equation (1). The pictured DAG describes the independence relationships within the model: A node is a random or fixed variable in the graph, a descendent node is defined as the node to which arrows point at any level in the in the graph, its parent nodes are those from which arrows point to the descendent node. Most nodes will thus be parents at one level and descendants of another level. In a DAG, each descendent node is thus conditionally independent of nodes preceding its parent node. Furthermore, plates (enclosing boxes in the graph) show recurring structures, such as parameters being defined for each individual $i$ or source $k$.

Starting from the top of the graph, $\gamma$ and $G_{0}$ are the priors for the mixing proportions $\pi$ and source specific parameters $\theta=\left(\theta_{1} \ldots \theta_{k} \ldots \theta_{K}\right)$ respectively. $\pi_{k}$ itself determines the probability $p\left(\xi_{i}=k\right)$. However, given the assignment $\xi_{i}=k$ and the source specific parameter vector $\theta$ the density for any $y$ does not depend on $\pi$. Similarly, given $\pi, \xi=\left(\xi_{1} \ldots \xi_{.} . . \xi_{N}\right)$ is independent of the prior $\gamma$.

This can be used when estimating parameters in the model: only the direct parents and descendants of any node will carry direct information regarding that node. One only needs to find conditional probabilities of each parameter given directly related nodes instead of specifying the full joint distribution over all parameters. Thus for the mixture model, we can simply sample repeatedly (and in any order) from $p\left(\pi_{k} \mid \gamma, \xi\right), p\left(\xi_{i} \mid y_{i}, \pi_{i}\right)$, $p\left(\xi_{i} \mid y_{i}, \boldsymbol{\pi}\right)$ and $p\left(\theta_{k} \mid \boldsymbol{y}, G_{0}\right)$. Often, some parameters can be integrated out of the model (i.e. the posterior distribution for all sources can be obtained analytically by integrating over $\theta$, which means that the Gibbs sampler only involves two sampling steps, those for $\pi$ and $\xi$ (Casella \& Robert 1996). 
Bayesian methods have a distinct advantage in that the breaking up of the joint probability of a model into conditional relationships and the sampling from resulting densities permits models of great complexity and flexibility - although the need for complexity needs to be carefully assessed for each model. Pella \& Masuda (2001) provided the first tailored Bayesian approach to mixture models for fisheries applications, which explicitly uses the prior density to select for informative alleles within a set of alleles. These authors also introduced the idea of directly modelling the baseline data as a sample from an unknown distribution rather than a collection of well-defined potential sources. Correctly estimating the effect of this uncertainty about the baseline sample in the resulting posterior distributions is a great advantage of the Bayesian method, and Koljonen et al. (2005) found that these methods usually outperform non-Bayesian methods. This is largely due to the unconditional (not to be confounded with the conditional distributions from the Gibbs sampler) nature of the model in which information is shared between the mixture and its baseline.

White et al. (2008) and Munch \& Clarke (2008) proposed methods tailored for otolith chemical data, but their analysis is rather different from that of Pella \& Masuda (2001). Munch \& Clarke (2008) propose a conditional model, in which fish are classified according to the posterior predictive probability of each fish in the mixture given the baseline. The posterior predictive distribution in Bayesian statistics is a distribution which integrates over the posterior distribution of unknown parameters (given the data) to predict the density of a new observation. This density can be written as $p\left(y_{i} \mid s_{i}=k, \boldsymbol{x}\right)=\int p\left(y_{i} \mid s_{i}=k, \boldsymbol{\theta}\right) p(\boldsymbol{\theta} \mid \boldsymbol{x}) d \boldsymbol{\theta}$, where $\boldsymbol{\theta}$ are the baseline parameters estimated from the baseline $\boldsymbol{x}$. Evaluating this integral numerically leads to a so-called collapsed Gibbs sampler (a method that also called Rao-Blackwellisation (Casella \& Robert 1996)), which enhances efficiency of the sampling procedure. Munch \& Clarke's method thus incorporates uncertainty from finite baseline samples, but still has the drawbacks of a necessarily known and fully sampled baseline. 


\subsection{Contributions of this thesis}

White et al. (2008) propose a method which does not really fit one of the above categories, it is more precisely a sub-form of both since it does not explicitly model the baseline as an entity different to the mixed sample. It is thus rather a clustering method which groups both the baseline and the mixed sample into homogenous groups. These authors also propose model selection to find the most likely number of sources in the mixture. While this approach provides a promising way towards finding the number of likely sources in a dataset, the underlying clustering model is limited in that it does not use the baseline directly to classify fish of unknown origin, but rather compares clusters obtained from the baseline and the mixed sample.

For genetic mixtures, Pella \& Masuda (2006) provide a way to directly infer un-sampled baseline contributions. This method is based on a Bayesian non-parametric prior formulation, using the Dirichlet Process model. This model produces a marginal posterior distribution over the number of sources in the baseline while assigning individuals to a set of unknown underlying distributions. It thus has all the advantages of their original model (Pella \& Masuda 2001), without the constraint to have sampled all contributing sources in the baseline. No comparable models currently exist for geochemical data and chapter 3 of my thesis aims at developing analogous methods which can be used with otolith chemistry.

Finally, no attempts have been made at a fully Bayesian analysis of the time resolved signatures obtained from otolith profiles. The lack of such methods for otolith data constitutes the motivation for chapter 5 of my thesis.

\subsection{Contributions of this thesis}

My thesis develops a combination of empirical, statistical and simulation approaches to analyse connectivity of marine fish metapopulations. Novel statistical methods for otolith chemical data 
obtained from fish otoliths (Figure 1.1) allow me to infer connectivity between local populations of common triplefin (Forsterygion lapillum, Figure 1.2) in three marine reserves in Cook Strait. Comparing these outcomes to newly developed simulation models of ocean currents provides insights into the mechanisms of dispersal in this system.

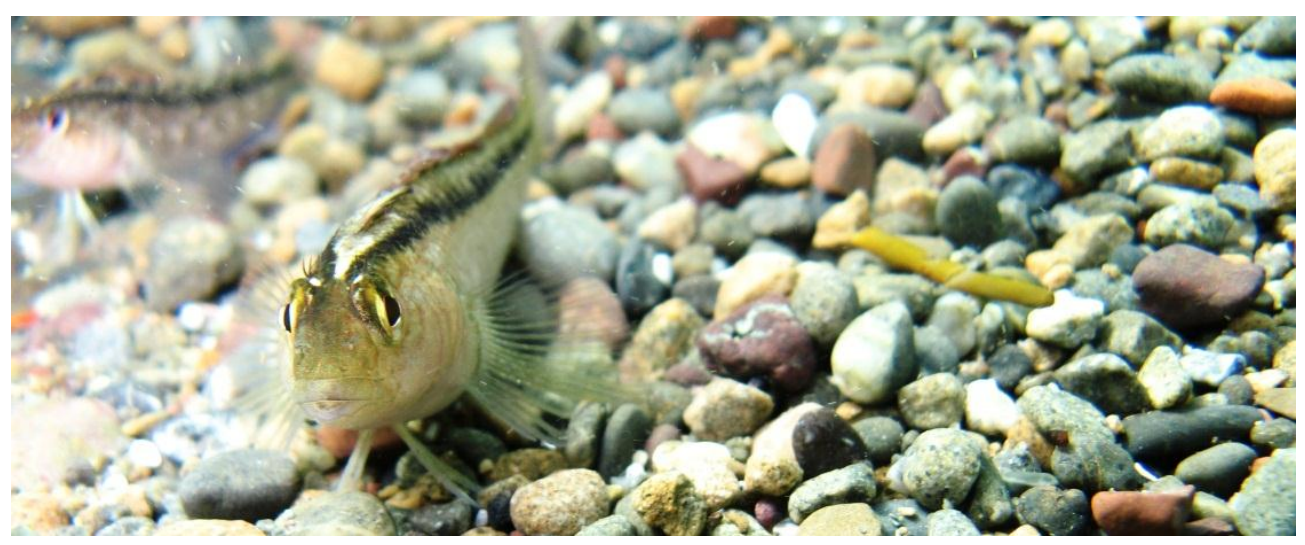

Figure 1.2: Picture of an adult common triplefin (Forsterygion lapillum). (C) P.Neubauer

In chapter two I start by examining otolith signatures of $F$. lapillum hatchlings from around Cook Strait, building an atlas (a baseline) of variability in otolith signatures. I develop statistical methods which examine the properties of this atlas and I discuss its value for connectivity studies. Examining variability over a range of scales and environments provides a complete picture of variability in otolith chemistry across a variety of conditions and thereby contributes to our understanding about the variability of these scales in marine environments. Additionally, this chapter forms the basis for the remaining empirical work in this thesis.

My third chapter tackles the important question of finding natal origins of fish when the geochemical atlas of potential natal sources is likely to be incomplete. Dirichlet process mixture models provide the basis for a solution to this limitation for geochemical studies: Fish recruits of unknown origin are no longer just assigned to a natal source in a fixed atlas or 


\subsection{Contributions of this thesis}

baseline, but are attributed to new sources if they are sufficiently different from the existing baseline. I develop this approach for clustering and classification with geochemical signatures and show its potential on the weakfish dataset of Thorrold et al. (2001). These statistical advances open new doors for the use of geochemical signatures in marine environments, where a complete characterization of natal sources is often impossible.

For Chapter 4 I collected F. lapillum recruits from three marine reserves in Cook Strait in order to explore whether connections between different regions and their reserves are evident. I employ the statistical methods developed in chapter 3 to find possible natal sources of these settlers and develop a simulation model which provides a potential mechanistic understanding of the dispersal patterns evidenced by otolith chemistry. To simulate dispersal, I outline a new refining grid model of hydrodynamics in Cook Strait, which provides a state-of-the-art tool to look at potential dispersal pathways in Cook Strait. A qualitative comparison of dispersal of passive particles in the model and empirical findings offers important scrutiny and validation of empirical results.

The fifth chapter builds on existing models to provide a general and flexible approach to modelling fish dispersal pathways from time series of otolith chemistry obtained by sampling the otolith chemistry longitudinally (along the growth axis). I illustrate how this general method can be applied in a variety of situations pertaining to a number of ecological questions often studied with this type of data. Additionally, I demonstrate how this approach provides a basis for model selection which can aid in deciding between competing hypotheses and to find appropriate models for a given dataset. The strengths and limitations of models based on this underlying framework are then illustrated with a set of simulation studies and a dataset of Chinook salmon (Miller et al. 2010), for which I investigate the size distribution at freshwater emigration based on otolith chemistry. 


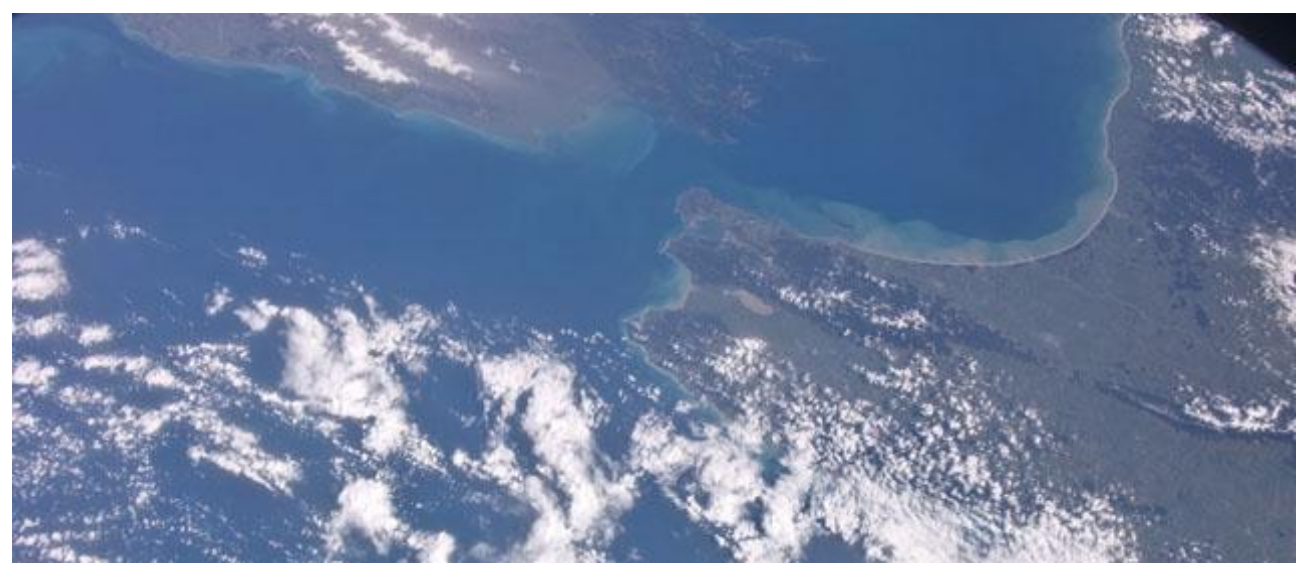

Figure 1.3: A Coastal boundary layer often forms from terrestrial runoff, visible in this NASA satellite image, motivating the model formulation in chapter 4.

The sixth and final data chapter applies the modelling approach developed in chapter five to chemical profiles across otoliths of the recruits collected for chapter 4 in order to get a better understanding of the mechanisms which underlie dispersal of F. lapillum in Cook Strait. To this end I develop a model which distinguishes between inshore and offshore water masses (Figure 1.3) in Cook Strait which provides insights into the dispersal traits of successful settlers in these populations. Idealized simulations with the adaptive grid ocean model developed in chapter 4 illustrate the benefits of these traits for maintaining metapopulation connectivity in the highly advective waters of Cook Strait.

I conclude my thesis by discussing the implications of my methodological and empirical findings in the context of marine metapopulations and the study of their connectivity and spatial structure. 


\section{Chapter 2:}

\section{Scale-dependent variability in hatchling otolith chemistry: implications for studies of population *}

\subsection{Introduction}

Patterns of connectivity among spatially discrete local populations have important consequences for the dynamics and behaviour of local populations and the larger metapopulation (Caley et al. 1996, Cowen et al. 2000). Nonetheless, our understanding of patterns of connectivity in coastal marine systems remains limited. Connectivity in many marine metapopulations is generally facilitated by dispersal of larvae. While larval durations can last weeks to months for some species, recent evidence suggests that long distance dispersal may be a relatively rare occurrence (Cowen et al. 2006), and self-recruitment could be important in some populations (see Swearer et al. 2002 for a review). Obtaining more complete knowledge of dispersal patterns in marine metapopulations is of critical importance if we hope to understand some of the key drivers of marine population dynamics.

In teleost fishes, otoliths (fish 'ear stones') are increasingly used to address dispersal questions (e.g. Campana \& Thorrold 2001, Thorrold et al. 2002, Elsdon \& Gillanders 2003, Shima \& Swearer 2009a). Otoliths are

\section{* A version of this chapter has been published in Marine Ecology Progress}




\subsection{Introduction}

known to act as environmental recorders during a fish's life (Campana \& Thorrold 2001): trace elements in the developmental environment are incorporated into growing otoliths and may reflect local environmental conditions (Campana 1999). Otoliths are metabolically inert, such that once deposited, trace element signatures form a permanent record. Trace element signatures can facilitate reconstruction of dispersal histories (e.g. Elsdon \& Gillanders 2003, Hamilton et al. 2008, Shima \& Swearer 2009a) and classification of individuals to putative natal source populations (e.g. Thorrold et al. 2002, Gillanders 2005, Ruttenberg et al. 2005).

Brophy et al. (2004) documented evidence for a peak of $\mathrm{Mn}$ in cores (the natal region) of otoliths in two species of fish, and Ruttenberg et al. (2005) extended these findings to six other species. The distinct manganese peak may thus serve as a marker of the core in otoliths sampled from older individuals, which may in turn act as a marker of natal conditions (Ruttenberg et al. 2005, Barbee \& Swearer 2007, Ruttenberg et al. 2008). Comparing trace element signatures from the otolith cores of settlers and/or recruits to an 'atlas' of trace element signatures (e.g., constructed from the otoliths of hatchlings, collected from known locations) could facilitate the assignment of settlers and/or recruits to natal populations of origin. The utility of this approach relies upon sufficient spatial variation in natal (core) otolith chemistry over the scale of this 'atlas' to enable settlers of unknown origin to be assigned to putative natal source populations with a high level of confidence.

Little is known about scales of variability of hatchling otolith trace element signatures in coastal environments. To date, most studies that have employed this approach in marine environments have focused on island chains (Warner et al. 2005, Ruttenberg \& Warner 2006, Ruttenberg et al. 2008) evidencing significant between-site variation within islands for trace element signatures. At larger spatial scales, among-site differences in signatures may overlap with those of other regions, and the discrimination of sites on these scales may prove problematic (Ruttenberg \& Warner 


\section{Chapter 2. Scale-dependent variability in hatchling otolith chemistry}

2006, Ruttenberg et al. 2008). In addition, natal signatures may be influenced by maternal or physiological effects (Campana 1999, Thorrold et al. 2006, S. Swearer unpublished data), such that clutches from different mothers within sites may differ markedly in signatures, and this source of variation could mask contributions from environmental signals to limit inferences relating to natal origins. Thus, before this overall approach can be successfully applied to address many questions of population connectivity, the geographical resolution and population specificity of hatchling otolith signatures needs to be established (Ruttenberg et al. 2008).

Additionally, statistical approaches to explore and utilize patterns in hatchling otoliths have been largely limited to statistical testing and linear discriminant analysis (LDA). Though LDA is generally practical for the assignment of recruits to possible sources (White \& Ruttenberg 2006), it may be limiting when the atlas is incomplete (Munch \& Clarke 2008, White et al. 2008). Alternatives have been suggested (Munch \& Clarke 2008) but their utility has not been empirically evaluated (but see White et al. 2008 for a related treatment of this problem). Furthermore, the proportion of correctly assigned individuals in a discrimination problem will diminish with an increasing number of classes (Smouse et al. 1982, Gillanders 2005, Barbee \& Swearer 2007). This has prompted some authors to consider spatially aggregating sites to counteract this effect (e.g. Gillanders 2002). While spatial aggregation of sites can be straightforward in simple geographical contexts (e.g., linear coastlines), the same is not necessarily true for more complex coastal geometries. In such contexts, no methods currently exist to find optimal groupings and therefore optimize the usefulness of hatchling otoliths as proxies for natal origins of fish.

The purpose of this chapter is twofold: (1) I explore patterns of variation in hatchling otolith signatures using a hierarchical (i.e., spatially nested) sampling design around Cook Strait, New Zealand, spanning a wide range of environmental conditions in the region. This approach enables me 


\subsection{Methods}

to evaluate sources of variation and infer plausible spatial scales of resolution that may be achievable using hatchling otolith microchemistry for my study system and species. (2) I describe and evaluate two approaches that may be used to improve the resolution and strength of inferences derived from trace element signatures. First, I employ a simulated annealing algorithm (e.g. Dupanloup et al. 2002) which statistically optimizes the spatial groupings of sites in order to increase the probability of correctly assigning individuals of unknown origin to their natal location. Second, I trial an 'exclusion test' approach (Cornuet et al. 1999, Munch \& Clarke 2008, Standish et al. 2008), which has been suggested for situations where not all possible source locations can be referenced within the natal atlas. I test the utility of this approach for my data by performing exclusion tests on otolith signatures of known origin to identify the spatial scales at which this approach can provide the most robust assignment of larval origins.

\subsection{Methods}

\subsubsection{Study Species \& Sampling}

My focal species for this study is the common triplefin Forsterygion lapillum, a small reef fish that is ubiquitous on relatively sheltered rocky reefs and tide pools around New Zealand, to a depth of $10 \mathrm{~m}$ (Clements 2003). Adults are strongly site attached, with a home range limited to a few square meters (Clements 2003, Wellenreuther et al. 2007). Females lay benthic eggs almost year-round within the study area (Wellenreuther \& Clements 2007, P. Neubauer, pers. obs.), typically on the underside of smooth cobbles, and nests are guarded by territorial males until hatching. Larvae hatch after $\sim 10$ days (P. Neubauer, unpublished data) and settle back to subtidal reefs in the region after a $\sim 52$ day pelagic larval duration (PLD) (Shima and Swearer 2009). I employed a hierarchical sampling design that included locations on both sides of the Cook Strait (the divide between the North and South Islands of New Zealand, Figure 2.1). The spatial extent 


\section{Chapter 2. Scale-dependent variability in hatchling otolith chemistry}

of the study area was informed by knowledge of mean currents in the region (Bowman et al. 1983, Heath 1986) and the probable local distribution of $F$. lapillum, derived from a boosted regression tree model of F. lapillum abundance in relation to important habitat variables (Smith 2008).

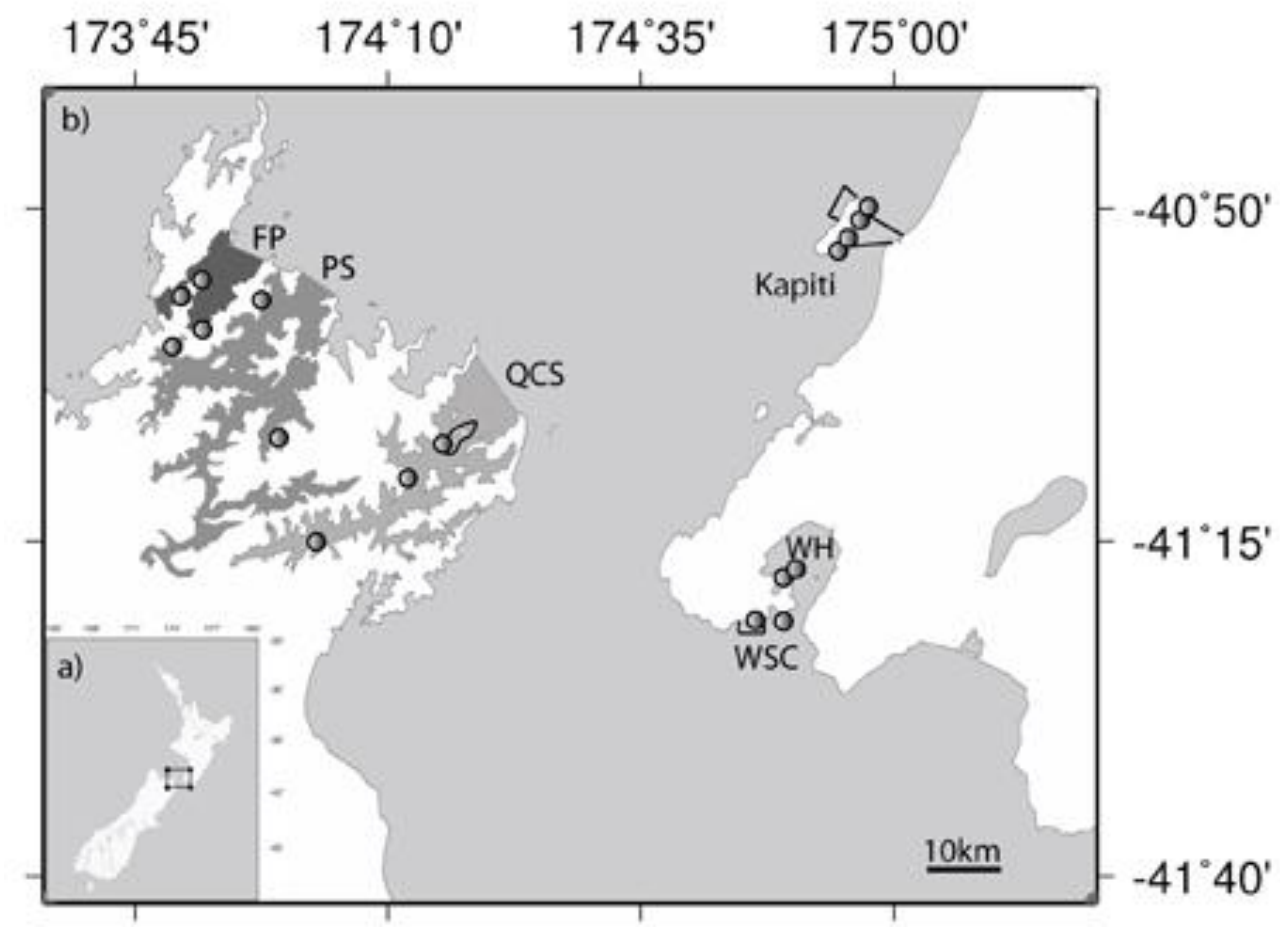

Figure 2.1: (a) Map of New Zealand and (b) the sampling domain. Sampling sites (grey circles) are scattered either side of Cook Strait. Black outlines mark borders of regional marine reserves. South Island regions within the Marlborough Sounds (QCS = Queen Charlotte Sound, PS = Pelorus Sound, FP = French Pass region) encompass three sites each. Kapiti Island was sampled at 4 sites along its eastern side, the south coast (WSC) of Wellington and Wellington's harbour (WH) regions consist of two sites each.

I collected eggs during the Austral summer from December 11th - 28th 2007 on the North Island and January 1st - 10th 2008 in the Marlborough Sounds region of the South Island. A total of 30 sites that seemed likely to sustain F. lapillum in substantial numbers were visited and I found eggs at 17 of these sites (Figure 2.1). From each site I attempted to collect at least 5 hatchlings from each of five discrete egg clutches. Since this was not 


\subsection{Methods}

always possible (due to the scarcity of clutches or the failure of some clutches to hatch), some of the sites in this study are represented by 3-4 clutches but 6-8 hatchlings per clutch to have comparable number of hatchlings from each site.

Clutches were collected using SCUBA or snorkelling in $<3 \mathrm{~m}$ depth. Cobbles or rocks containing late-stage eggs (indicated by embryos with visible eye spots) were collected by hand and placed in a sterile plastic bag along with $\sim 2 \mathrm{~L}$ of water from the location of collection. On shore, the contents of bags were emptied into clean PVC buckets with lids, such that individual clutches were submerged and maintained in ambient water from the natal site of collection. All but two clutches (both from one site at Kapiti Island) hatched within $30 \mathrm{~min}$ after collection. The remaining two clutches were kept in the lab (in sea water from the site of collection, supplemented with bubbled air) for 36 and 48h, respectively. These clutches were excluded from later statistical analysis because signatures were substantially different from clutches that hatched immediately at this site, indicating possible handling effects. After hatching, all larvae were transferred into $0.2 \mathrm{ml}$ Eppendorf tubes containing 95\% analytical grade ethanol, and transported to the lab for otolith extraction and analysis.

\subsubsection{Otolith extraction, preparation and analysis}

Hatchling otoliths were extracted individually from fish, in a randomized order, under a laminar flow fume hood. To further minimise the risk of contamination (which could confound trace element signatures measured within otoliths), all material used during extraction and preparation of the otoliths was acid rinsed for $24 \mathrm{~h}$ in $6 \mathrm{~N} \mathrm{HCL}$, then rinsed three times in Millipore 18.2 $\mathrm{M} \Omega$ water prior to use. Individual hatchlings (i.e., larvae) were placed in a droplet of Millipore water on a clean slide and a sagittal otolith was extracted from each larva. Otoliths were subjected to cleaning and rinsing baths as in Barbee \& Swearer (2007) before being embedded in resin on a gridded slide. A Varian Laser Ablation Inductively Coupled Plasma Mass Spectrometer (LA-ICPMS) at the University of 


\section{Chapter 2. Scale-dependent variability in hatchling otolith chemistry}

Melbourne was used to analyse eleven trace elements contained within the hatchling otoliths $\left({ }^{7} \mathrm{Li},{ }^{11} \mathrm{~B},{ }^{24} \mathrm{Mg},{ }^{31} \mathrm{P},{ }^{34} \mathrm{~S},{ }^{55} \mathrm{Mn},{ }^{63} \mathrm{Cu},{ }^{66} \mathrm{Zn},{ }^{88} \mathrm{Sr},{ }^{138} \mathrm{Ba}\right.$, ${ }^{208} \mathrm{~Pb}$ ). Otoliths were ablated vertically in a deepening pit resulting in a series of readings from the surface of the otolith through the core. For further details of the extraction-, preparation- and analytical methods, see Barbee \& Swearer (2007).

All data were post-processed with calculations being identical to those used in Barbee \& Swearer (2007). Time series resulting from this processing were inspected visually to check for signs of surface contamination. Specifically, I inspected the first and last readings corresponding to the otolith surface, looking for elevated peaks in elements such as $\mathrm{Zn}$ and $\mathrm{Pb}$, which are prone to contamination. 10 scans to either side of the manganese peak (interpreted as the otolith primordium) were averaged to obtain the natal trace element signatures for subsequent statistical analyses.

\subsubsection{Statistical analysis}

\subsubsection{Descriptive statistics, significance testing and discrimination}

I employed a nested model to explore patterns and sources of variation in trace element signatures across all levels of the hierarchical sampling design. 'Clutches' (Level 1 - random effect) were nested within 'sites' (Level 2 - random effect), which were in turn nested within the different (a priori defined) 'regions' (Level 3 - fixed effect) distributed across both main 'islands' (Level 4 - fixed effect) of New Zealand, separated by the Cook Strait. Note that by making such arbitrary groupings, I make implicit assumptions about processes that govern geographical patterns in otolith chemistry - i.e. I assume that all sites within a region are influenced by a region specific generative process. I further assume that the regions represented here cover all possible spawning locations (motivating the fixed effect for this level). I address this issue in more detail below. I performed non-metric multidimensional scaling (MDS) to visually 


\subsection{Methods}

inspect similarities at different levels of the hierarchy (e.g. sites in the sampling domain, and clutches that hatched at different times - see previous section 'Study Species \& Sampling').

To test significance at different levels of the sampling design I opted for permutation based multivariate analysis of variance (Anderson 2001). All data were standardized to unit variance. Standardized data were permuted within levels of the nested hierarchy so as to keep the higher level fixed while permuting the next lower level to avoid testing against variability at other levels (Anderson 2001). All levels were tested with 9999 permutations. Significance testing was conducted using PRIMER software with PERMANOVA add-on (Anderson 2001, Anderson et al. 2008). As this was a mixed model, components of variation were calculated as sums-ofsquares divided by degrees of freedom for fixed effects and as variance components derived from expected mean squares for random effects (Anderson et al. 2008).

To facilitate comparisons with earlier studies, I used linear discriminant analysis (LDA) of log-transformed data to discriminate amongst classes in levels of the sampling hierarchy. Linear discriminant analysis (LDA) has been widely used to classify and assign hatchling otoliths to potential source populations, though other classifiers are preferred in certain situations (Mercier et al. in press). The usefulness of the hatchling otolith 'atlas' is ultimately measured by its ability to successfully discriminate amongst putative source populations. I chose to evaluate the performance of this method based on the overall classification success of hatchling otoliths (with known geographic origins), using "leave-one-out" cross validation as an indicator of performance of the technique at different levels of the sampling hierarchy. Since this measure represents an estimate from the sample at hand, I used 1000 parametric bootstrap samples (drawing from a multivariate normal distribution with mean and covariance matrix of log-transformed data) for each source to calculate confidence limits of cross validation results as $2.5 \%$ and $97.5 \%$ quantiles of the 


\section{Chapter 2. Scale-dependent variability in hatchling otolith chemistry}

bootstrap distribution. I also calculated the relative contribution of each element to class separation by calculating their contribution to the $\mathrm{F}$ ratio (the ratio of between class variability to within class variability).

To visually illustrate the signal to noise ratio of levels of the hierarchy, I plotted the mean Mahalanobis distance at each level, which is the Euclidian distance between group means (the 'signal') weighted by intragroup variance (the 'noise'), against number of groups and expected assignment success (Smouse et al. 1982, White \& Ruttenberg 2006). All analyses in this and the following sections were conducted using the statistical computing language R (R Development Core Team 2007).

\subsubsection{Optimal grouping of sites by simulated annealing}

I initially imposed a hierarchical spatial structure on the sampling programme (e.g., defining discrete regions within islands, etc.), but this structure might not represent natural underlying scales of variability in trace element signatures. To identify optimal, non-a priori groupings of sites that might improve the allocation success of unknown signatures (and thus, improve the power of the approach), I used a simulated annealing (SA) algorithm. SA is a general optimization heuristic that can locate global optima in combinatorial problems. The procedure used here essentially follows a simple SA algorithm (e.g. Burkard \& Rendl 1984). At each iteration of the algorithm, a spatial configuration, which conforms to constraints in a contiguity matrix, is proposed (only adjacent sites can be grouped). Problem related constraints were added where needed (see also Dupanloup et al. 2002 for a related simulated annealing algorithm in genetics) 


\subsection{Methods}

The procedure can be summarized as follows:

1. Propose any starting configuration, such as the a priori groupings

2. Randomly choose to either merge two adjacent sites/clusters of sites or to remove a site from a cluster. This involves a Bernoulli trial (merge/split) with probability 0.5 followed by two multinomial trials $T=\{i, j\}$ with probability $\frac{1}{N_{T}}$ (sites or clusters $I$ and $J$ to merge or site $J$ to remove from cluster $I$, where $I, J$ are the outcomes of trials $i$ and $j$ respectively), where $N_{T}$ is the number of possible choices in each trial. Note that $N_{T}$ for trial $J$ is dependent on the outcome of trial $I$.

3. Calculate the value of the cost function $E$. In this case this is the expected assignment accuracy for the new configuration (Smouse et al. 1982). Accept the new grouping if $E_{s \in S}>\max \left(E_{1: s-1}\right)$, else accept with probability proportional to the difference of $E_{s}-E_{s-1}=\Delta E$, namely $\exp \left(\frac{\Delta E}{\tau}\right)$.

4. Return to step 2 for $S$ iterations

The variable $\tau$ is called the temperature with analogy to related physical problems. It is decreased (logarithmically in this case) with the number of iterations, such that, at the initiation of the procedure, suboptimal configurations are accepted with high probability. In later iterations, suboptimal configurations become increasingly unlikely. This progressive decrease in the acceptance of suboptimal configurations enables the algorithm to overcome local optima and identify a global optimum. A more conventional clustering approach would yield a single (but potentially sub-optimal) cluster configuration; the SA approach facilitates a more thorough exploration of all possible configurations to increase the likelihood of arriving at a globally optimal solution.

I ran the algorithm with three different additional constraints: (1) any spatial configuration of sites was allowed except re-grouping across islands 


\section{Chapter 2. Scale-dependent variability in hatchling otolith \\ chemistry}

- this restriction was always applied since I felt that the reasonable discriminatory power at this scale (see results) made regroupings across islands unnecessary and counter-productive - (2) the optimal solution must differentiate at least four groups (SA4) and (3) the optimal solution was biased in favour of many small groupings (SA MS). For this last constraint the cost function was modified by multiplying the expected classification success by the number of groupings (favours many groups) and then dividing by the size of groups (favours small groups). Note that by introducing a constant in this fraction, the cost function can be arbitrarily modified to give selection criteria that favour resolution or overall classification success. Post-hoc permutational multivariate analysis of variance was performed on groupings obtained from the different constraint scenarios.

\subsubsection{Exclusion test}

For some applications and research questions, it may not be possible or necessary to construct a definitive atlas of natal signatures (e.g. Elsdon et al. 2008). In such instances, it may still be useful to differentiate between individuals that likely originated from a particular location (e.g., a natal source population within a marine reserve) versus all other locations. To test whether such questions can be addressed with hatchling otoliths, I applied an 'exclusion test' framework to a subset of the data. Here, the focus is on sub-groups within the sampling area, and I applied a statistical approach to evaluate the probability that individuals of unknown origin do not belong to a grouping of interest (Cornuet et al. 1999, Barbee \& Swearer 2007, Munch \& Clarke 2008, Standish et al. 2008). Using log-transformed trace element signatures from hatchling otoliths, I calculated Mahalanobis distances from the centroid of the focal group to samples known to originate from all other sites (i.e., individuals with natal origins other than the focal population). The distribution of squared Mahalanobis distances from a multivariate normal distribution follows a $\chi^{2}$ distribution with $p$ degrees of freedom, where $p$ is the dimension of the data. This 


\subsection{Results}

distribution can thus be used as the null distribution and one can look up the critical value ( $\alpha=0.05$ for instance) for belonging to a focal group/site (represented by this distribution) in a standard $\chi^{2}$ table. Samples whose squared Mahalanobis distance to the centroid of the focal group/site is above this critical value can then be considered to have originated from a source outside the focal population (Munch \& Clarke 2008).

To illustrate the potential utility of this approach, I applied this exclusion test to a subset of my data that included individual sites and groups of sites within Pelorus Sound and Kapiti Island (two regions of high and low separation, respectively). Additionally, I investigated exclusion potential from two marine reserves in my dataset: Long Island Marine Reserve in the Marlborough Sounds and Island Bay (Taputearanga Marine Reserve) on the Wellington south coast. Since these results may be an artefact of sampling (i.e., unsampled sites may have similar signatures to sampled ones), I repeated these tests for one case (Pelorus Sound), each time dropping 2, 5 or 10 randomly selected sites from the test. This approach enabled me to evaluate the power of the exclusion potential for this site or subset of sites for a given sampling effort.

\subsection{Results}

\subsubsection{Descriptive statistics, significance testing and discrimination}

Table 2.1 gives statistics relevant to chemical analyses, detection limits, as well as precision estimates. Only $\mathrm{Li}$ and $\mathrm{Pb}$ were routinely below detection limit, and neither element contributed to differences between sites (Table 2.2). I identified significant variation among clutches within sites $(P=0.0001)$, among sites within a priori defined regions $(P<0.0001)$ and between islands $(\mathrm{P}=0.0002) \quad$ (Table 2.2a). The a priori regions within islands were not significantly different $(P=0.43)$, and their difference explained $0.2 \%$ of the overall variance in the dataset. Small-scale differences in trace element signatures explained the largest portion of the 
variance, with sites accounting for $7.9 \%$, whereas clutch differences within these locations explained $11.8 \%$. A total of $72.8 \%$ of the total variance in the between signatures remained unexplained by the terms in the model (i.e., variation among eggs within clutches).

Jackknife cross validation on LDA with equal prior probabilities for all classes indicated that $76.2 \%$ ( $\mathrm{Cl} 74.4 \%-78.9 \%)$ of hatchlings could be correctly assigned to their natal island despite overlap of distributions (Table 2.2a, Figure 2.2a). A total of $34.9 \%$ ( $\mathrm{Cl} 33.4 \%-43.8 \%$ ) of hatchlings were correctly assigned to the pre-defined regions (Table 2.2a), and the separation between these regional groupings was relatively poor (Figure $2.2 \mathrm{~b}, \mathrm{c})$. Sites of natal origin were correctly assigned for only $32.5 \%(\mathrm{Cl}$ 29.7\%-33.2\%) of hatchlings (Table 2.2a) in the total dataset, but showed the highest signal to noise ratio (Figure 2.3) of all levels in the hierarchy. Additionally, sites from different regions were often more similar than sites within regions (Figure 2.4); i.e., sites often overlapped in their distributions. When restricting the domain to just a single region, I could correctly assign $79.8 \%(\mathrm{Cl} 73.1 \%-86.0 \%)$ of hatchlings to their natal site of origin within Pelorus Sound (Table 2.2b, Figure 2.2d), and 56.4\% (Cl 50.1\%-63.5\%) within Kapiti (Table 2.2b), even though trace element signatures for the sites within Kapiti were not significantly different. 
Table 2.1: Distribution of trace element concentrations (in mol per mol Ca; note multiplier for each element column) relative to detection limits. Given are elemental concentrations for the quantiles that bracket $95 \%$ of all observations made from sampled otoliths, and the median value. Detection limits (DL in mol per mol Ca) and external precision estimates (consistency standard, CS) for analyses of hatchling otoliths by LA-ICP-MS. DL estimates based on 70 blank analyses. Estimates of external precision are given in relative standard deviations (\%RSD) and are based on 31 blocks of samples (a block is a series of samples bracketed by standards), treating the NIST (National Institute of Standards and Technology) standard that is just below the standard used for calibration for each element as unknown and calculating the Element:Ca ratio for this standard for each block of samples.

\begin{tabular}{|c|c|c|c|c|c|c|c|c|c|c|c|c|}
\hline \multicolumn{2}{|l|}{ Element } & ${ }^{7} \mathrm{Li}\left(\times 10^{-6}\right)$ & ${ }^{11} \mathrm{~B}\left(\times 10^{-3}\right)$ & ${ }^{24} \mathrm{Mg}\left(\times 10^{-3}\right)$ & ${ }^{31} \mathrm{P}\left(\times 10^{-3}\right)$ & ${ }^{34} S\left(\times 10^{-3}\right)$ & ${ }^{55} \mathrm{Mn}\left(\times 10^{-6}\right)$ & ${ }^{63} \mathrm{Cu}\left(\times 10^{-6}\right)$ & ${ }^{66} \mathrm{Zn}\left(\times 10^{-6}\right)$ & ${ }^{88} \mathrm{Sr}\left(\times 10^{-3}\right)$ & ${ }^{138} \mathrm{Ba}\left(\times 10^{-6}\right)$ & ${ }^{208} \mathrm{~Pb}\left(\times 10^{-6}\right)$ \\
\hline \multirow{3}{*}{$\begin{array}{l}\text { Sample } \\
\text { quantiles }\end{array}$} & 0.025 & 4.50 & 0.14 & 0.40 & 0.69 & 0.54 & 175.27 & 0.34 & 6.63 & 1.69 & 3.73 & $5.0 * 10^{-4}$ \\
\hline & 0.5 & 8.20 & 0.23 & 0.54 & 0.87 & 0.67 & 427.35 & 1.77 & 33.24 & 2.04 & 12.09 & 0.046 \\
\hline & 0.975 & 34.62 & 0.45 & 0.83 & 1.29 & 0.78 & 1108.58 & 25.08 & 295.45 & 2.67 & 48.86 & 0.41 \\
\hline \multicolumn{2}{|l|}{$\mathrm{DL}$} & 11.44 & 0.030 & 0.029 & 0.095 & 0.14 & 3.82 & 1.57 & 2.36 & $4.1^{*} 10^{-4}$ & 0.031 & 0.096 \\
\hline \multicolumn{2}{|l|}{ CS } & 8.54 & 9.14 & 7.83 & 13.27 & 6.16 & 7.32 & 8.37 & 8.26 & 7.48 & 7.77 & 7.21 \\
\hline \multicolumn{2}{|l|}{ NIST(CS) } & 614 & 614 & 612 & 612 & 612 & 612 & 614 & 614 & 612 & 614 & 614 \\
\hline
\end{tabular}



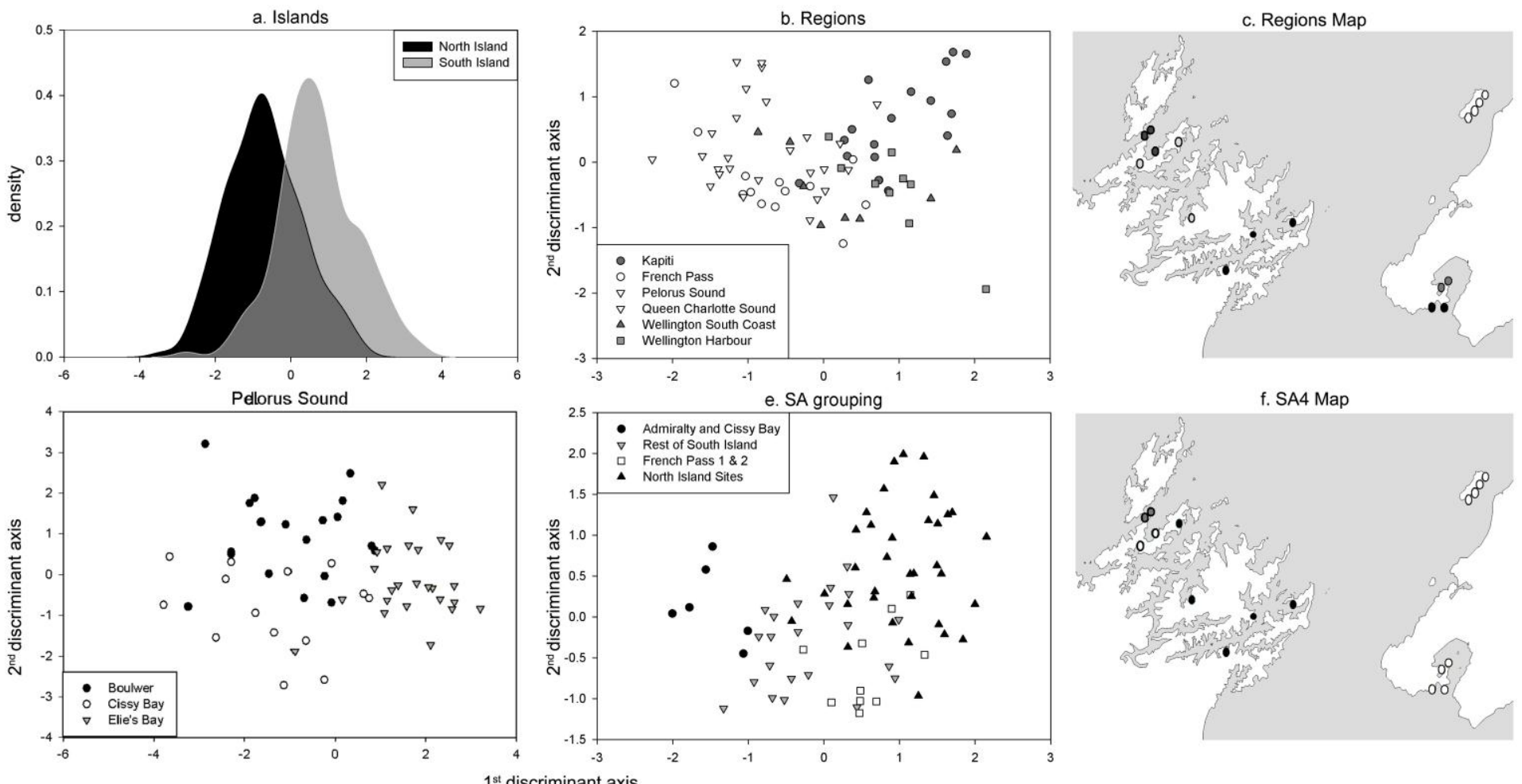

Figure 2.2: Top left to bottom right panel: (a) Kernel smoothed histograms of trace element signatures from the two main Islands along the linear discriminant function. (b) Regional differences along the first two linear discriminant axes. Only clutch means are plotted for clarity. (c) Map of a priori defined regions; symbols of like shading define discrete regions. (d) Site differences in Pelorus Sound. (e) Four groups obtained with simulated annealing (SA4) to optimize allocation success in a linear discriminant analysis; clutch means are shown along the first two axes. (f) Map of groupings obtained by Simulated Annealing (SA); symbols of like shading define groupings. 


\subsection{Results}

Table 2.2: Variance explained (as sums-of-squares divided by appropriate degrees of freedom for fixed effects and variance components for random effects) and $P$ values (Proportion of permuted pseudo $F$ values (PsF) $>$ model pseudo $F$ ) from nested permutational MANOVA for: (a) a priori groupings, (b) sites within regions and (c) optimal groupings obtained with simulated annealing (SA 4 for conditions specifying a maximum number of four groups, SA MS for conditions favouring small groups). Mean CV shows overall mean percentage of correctly assigned individuals using leave-one-out cross validation. Elements are the three most important elements in the LDA in terms of their contributions to separation amongst groups as indicated by their relative contribution to the F statistic (in brackets).

\begin{tabular}{|c|c|c|c|c|c|c|c|}
\hline Term & Levels & Variance & $\mathrm{P}(>\mathrm{PsF})$ & & Element & & $\begin{array}{c}\text { Mean } \\
\text { CV }\end{array}$ \\
\hline \multicolumn{8}{|c|}{ a) A priori groupings } \\
\hline Island & 2 & $7.3 \%$ & 0.0002 & $\begin{array}{c}\mathrm{Sr} \\
(0.35)\end{array}$ & $\begin{array}{c}\text { B } \\
(0.23)\end{array}$ & $\begin{array}{c}\mathrm{Mg} \\
(0.13)\end{array}$ & $76.2 \%$ \\
\hline Region[Island] & 6 & $0.2 \%$ & 0.43 & $\begin{array}{c}\mathrm{Sr} \\
(0.30)\end{array}$ & $\begin{array}{c}\text { B } \\
(0.19)\end{array}$ & $\begin{array}{c}S \\
(0.13)\end{array}$ & $34.9 \%$ \\
\hline Site(Region[Island]) & 17 & $7.9 \%$ & 0.0001 & $\begin{array}{c}S r \\
(0.23)\end{array}$ & $\begin{array}{c}S \\
(0.21)\end{array}$ & $\begin{array}{c}\text { B } \\
(0.20)\end{array}$ & $32.5 \%$ \\
\hline Clutch[Site(Region[Island])] & 74 & $11.8 \%$ & 0.0001 & & - & & - \\
\hline Residual & & $72.8 \%$ & & & - & & - \\
\hline \multicolumn{8}{|c|}{ b) Sites within Regions } \\
\hline Kapiti & 4 & $10.7 \%$ & 0.13 & $\begin{array}{c}S \\
(0.28)\end{array}$ & $\begin{array}{c}\mathrm{Mg} \\
(0.16)\end{array}$ & $\begin{array}{c}\mathrm{Sr} \\
(0.15)\end{array}$ & $56.4 \%$ \\
\hline Residual & & $89.3 \%$ & & & - & & - \\
\hline Pelorus & 3 & $9.6 \%$ & 0.0001 & $\begin{array}{c}S \\
(0.23)\end{array}$ & $\begin{array}{c}\mathrm{Sr} \\
(0.22)\end{array}$ & $P(0.17)$ & $79.8 \%$ \\
\hline Residual & & $90.4 \%$ & & & - & & - \\
\hline \multicolumn{8}{|c|}{ c) After Simulated Annealing (SA) } \\
\hline SA 4 [Island] & 4 & - & 0.55 & $\begin{array}{c}S \\
(0.30)\end{array}$ & $\begin{array}{c}\mathrm{Sr} \\
(0.22)\end{array}$ & $\begin{array}{c}B \\
(0.20)\end{array}$ & $51.7 \%$ \\
\hline SA MS [Island] & 9 & - & 0.69 & $\begin{array}{c}\mathrm{Sr} \\
(0.28)\end{array}$ & $\begin{array}{c}\text { B } \\
(0.20)\end{array}$ & $\begin{array}{c}S \\
(0.20)\end{array}$ & $35 \%$ \\
\hline
\end{tabular}




\section{Chapter 2. Scale-dependent variability in hatchling otolith chemistry}

Discrimination at all scales was driven by a suite of elements, the most important of which were Sr, B and S for global discrimination. Narrowing the scope to sites within regions revealed that elements such as $\mathrm{S}, \mathrm{P}$ and Mg determined the discrimination at this scale. No elements generally dominated the LDA over all analyses.

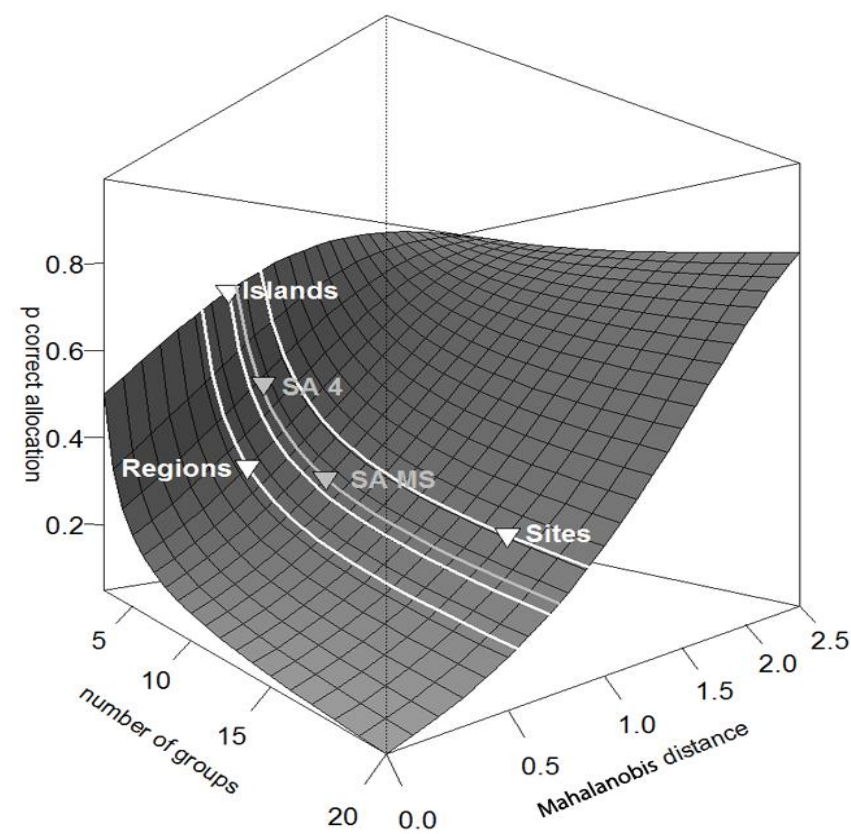

Figure 2.3: Perspective plot showing predicted probability (p) of correct assignment in a linear discriminant analysis in relation to number of groups and inter-group Mahalanobis distance. With no separation between groups and equal group sample sizes, all else being equal, assignment success in a linear discriminant analysis will be $1 /$, with I the number of groups in the analysis. With increasing distance between group centroids, assignment success increases nonlinearly. At the 'site' level of the sampling hierarchy I obtained the highest signal to noise ratio (as indicated by higher average distance), yet overall success was low due to the elevated number of groups (17 sites). Groupings from simulated annealing (SA4 and SA MS) achieved better signal to noise ratios then a priori groupings of sites into 'regions'. 


\subsection{Results}

\subsubsection{Optimal grouping of sites by simulated annealing}

Simulated annealing for the two different cost functions produced distinct grouping patterns which were found repeatedly on different runs of the algorithm. With no restrictions, the algorithm optimizing for overall allocation success consistently converged to two groups representing the two islands. When restricting to at least 4 groups, all sites within the North Island grouped together as a single entity, while the South Island sites formed 3 distinct groupings. This spatial configuration (informed by the data rather than ad hoc by us as observers) resulted in $51.7 \%(\mathrm{Cl} 49.8 \%$ $58.1 \%$ ) of hatchlings correctly assigned to their natal regions (Table $2.2 \mathrm{c} \&$ Fig.2.2e, f). Varying the cost function to favour more groups produced six groupings and left three sites ungrouped. Thus a total of nine groups were entered in the LDA which correctly allocated $35.0 \%$ (Cl $33.3 \%-40.3 \%)$ of hatchlings to natal regions.

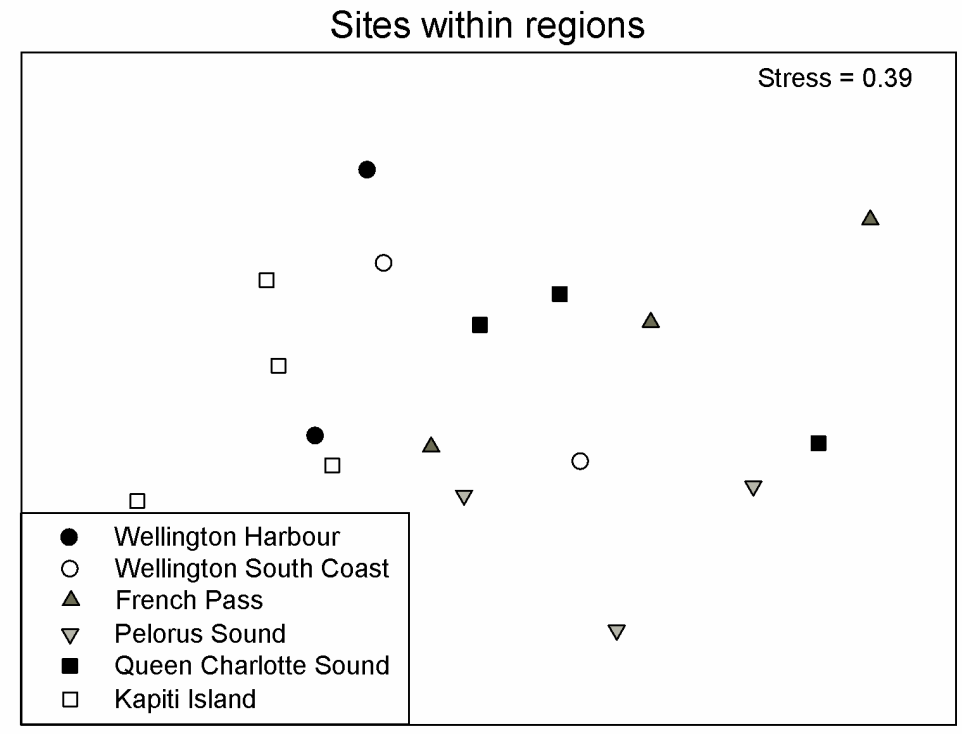

Figure 2.4: Non-metric multi-dimensional scaling of sites within the total sampling domain. A priori defined regions are represented by symbols of like colour and shape. 


\section{Chapter 2. Scale-dependent variability in hatchling otolith chemistry}

\subsubsection{Exclusion test for groups of sites}

Exclusion tests for Pelorus Sound rejected 98\%, 88\% and $61 \%$ of hatchlings from all other sites for the three sampled sites in this embayment. $76 \%$ of signatures from other sites could be correctly excluded from Kapiti Island sites on average. For the sites of Long Island Marine Reserve and Island Bay (Taputearanga Marine Reserve), I could exclude $95 \%$ and $85 \%$ of remaining signatures in the dataset respectively (Figure 2.5a,b). When pooling sites within regions for the test, the percentage rejected decreased dramatically, only $39 \%$ and $23 \%$ of the remaining hatchlings could be rightfully excluded for Pelorus Sound and Kapiti (Figure 2.5c) respectively.

Repeating these tests for Pelorus Sound sites showed that variability in estimates of our ability to correctly exclude signatures from other sites was directly related to sampling effort (Table 2.3 ): the variability in my results increased with the number of sites discarded. For one of my sites for instance (site 2 in Table 2.3), I estimated that about $88 \%$ of foreign signatures could be excluded from this site based on the remaining dataset or 16 remaining sites. Repeating the test with only 6 remaining sites resulted in estimates as high as $99 \%$ of foreign signatures correctly excluded (or as low as $79 \%$ depending on which sites made up the final sample) (Table 2.3).

Table 2.3: Exclusion test results in per cent of hatchlings correctly excluded from the focal location using full and truncated datasets with 2,5 and 10 randomly chosen sites dropped from the analysis. For the truncated scenarios only minimum and maximum percentages out of 20 trials are shown.

\begin{tabular}{|ccccc|}
\hline Sites omitted & 0 & 2 & 5 & 10 \\
\hline \hline Pelorus All & 39 & $35-41$ & $29-44$ & $22-52$ \\
Site 1 & 98 & $98-99$ & $98-100$ & $96-100$ \\
Site 2 & 88 & $87-92$ & $83-95$ & $79-99$ \\
Site 3 & 61 & $59-65$ & $56-67$ & $50-79$ \\
\hline
\end{tabular}




\subsection{Results}

a)

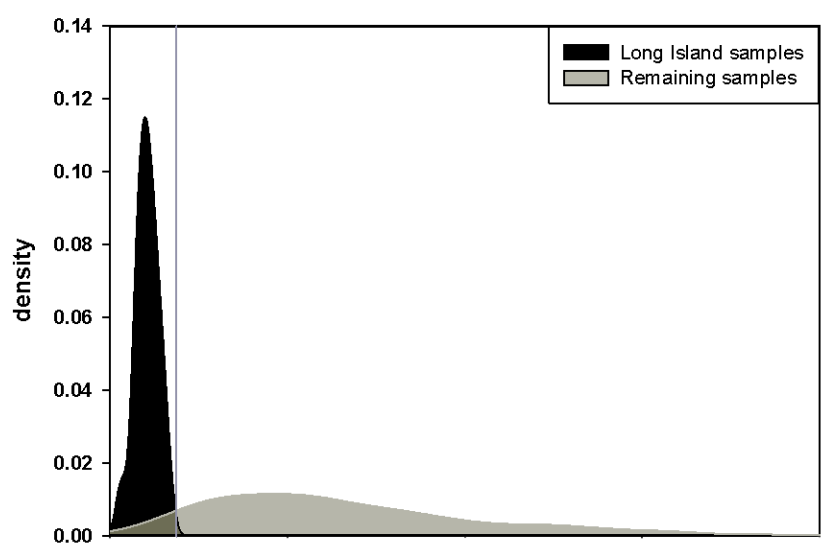

b)
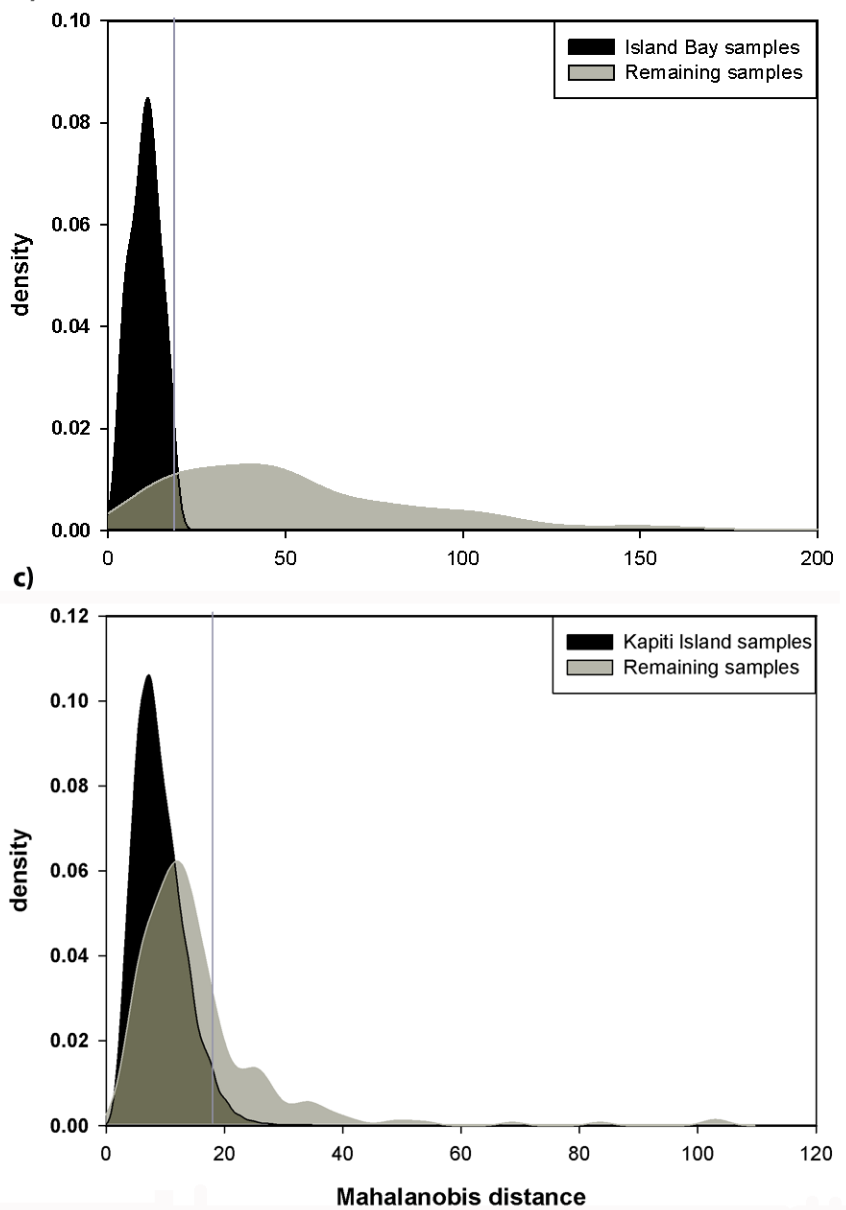

Figure 2.5: Kernel-smoothed histograms (black) of Mahalanobis distances to the centroid of the focal site, from chemical signatures of individual fish spawned at: (a) Island Bay (Taputearanga marine reserve) on the Wellington South Coast; (b) Long Island marine reserve in Queen Charlotte Sound; (c) Kapiti region (pooled across sites within this region). The light polygons are Mahalanobis distances of remaining signatures in the dataset to the centroid of the focal site, the black line is the $95 \%$ cut-off value for belonging to the site (i.e., the portion of the light histogram to the left of this line represents signatures incorrectly classified to the focal population, at $\alpha=0.05$ ). 


\section{Chapter 2. Scale-dependent variability in hatchling otolith \\ chemistry}

\subsection{Discussion}

\subsubsection{Scaling of variability in hatchling otolith signatures}

A complete description of connectivity in marine metapopulations requires an understanding of all pair-wise larval exchanges between local populations. Inferring such patterns from hatchling otolith microchemistry depends upon unique trace element signatures across all sites within a domain of interest (and across all sites in a natal atlas when recruits of unknown origin are to be assigned to putative natal populations).

Despite the dissimilarity in geography, I observed scales of variability in hatchling otolith signatures comparable to those found for the Galapagos Islands and Hawaiian Islands (Ruttenberg \& Warner 2006, Ruttenberg et al. 2008) as well as those found along the Oregon coast (Miller 2007a). Though there were clear differences in trace element signatures of hatchlings across small scales (e.g., between clutches within sites, and between sites within regions) and large scales (e.g. between islands), I failed to observe significant variability in signatures among regions within islands. I used simulated annealing to redefine regions post hoc, and while this improved the overall power of this approach (i.e., capturing additional variability at regional scales), differences among regions within islands were still not particularly strong. This suggests that, for this system, much of the variability in trace element signatures recorded within the otoliths of hatchlings is explained by processes occurring at large spatial scales (e.g., temperature/salinity gradients between north and south islands) and at smaller spatial scales (e.g., variation in trace elements among sites and/or females). When I restrict the focus to individual regions for instance, sites are relatively distinct: within the Pelorus Sound embayment and for the offshore island of Kapiti, sites can be discriminated amongst with some confidence, despite some sites (e.g., Kapiti) being relatively close geographically and not significantly different from each other. 


\subsection{Discussion}

The a priori regional groupings reflected prior beliefs about processes that may contribute to spatial variation in trace element signatures, such as 'polluted' and 'pristine' river inputs for Wellington Harbour and Pelorus Sound respectively. However, my results suggest that this variation (and presumably also the processes that shape this variation) act on scales that differ from the a priori defined regions. The fact that the data do not allow for accurate estimation of natal origins at the regional level of the natal reference atlas may seem an important limitation of the hatchling otolith approach for this system. I suggest that this limitation could be addressed by adopting a Bayesian approach (Munch \& Clarke 2008) that incorporates prior knowledge of, e.g., local hydrodynamics, genetic information and distributional maps of species. Explicitly and probabilistically integrating such supplementary information could reduce uncertainty around natal sources by adding confidence in assignments.

My analyses suggest that clutches collected from a common site vary substantially in trace element signatures, and this is consistent with reports from other systems (Warner et al. 2005, Ruttenberg \& Warner 2006, Ruttenberg et al. 2008). These differences possibly represent differential maternal provisioning, and/or they may reflect fine scale variability in the environment (Ruttenberg et al. 2005, Chittaro et al. 2006). Some trace metals (e.g., $\mathrm{Zn}$ and $\mathrm{Mn}$ ) act as cofactors in enzymatic reactions of phytoplankton and may thus exhibit small scale variability with respect to depth or sources of input and associated planktonic communities (Morel \& Price 2003).

A suite of elements was useful to discrimination at all scales. Since no one element dominated the F statistics at any scale, or even over multiple scales, my data suggest that multi-elemental signatures may enhance discrimination. Variation in many of these elements may reflect differences in water chemistry (e.g. Sr, Ba; (Elsdon \& Gillanders 2005, Walther \& 


\section{Chapter 2. Scale-dependent variability in hatchling otolith chemistry}

Thorrold 2006) whereas variation in other elements may indicate differences in physiology (perhaps mediated by spatial variation in water temperature, genetic variation), or differential maternal investment; (e.g. Chittaro et al. 2006, Thorrold et al. 2006). I note that a large part of the variance in the dataset remains unexplained by the model. This may be due to an inadequate model (e.g. the model structure does not reflect the structure in the data), measurement noise, non-obvious contamination and/or residual variation between hatchlings from a common clutch. Additional research is needed to determine the underlying sources of observed variability in trace element signatures across different spatial scales (see also Elsdon et al. 2008).

Furthermore, my data do not allow me to examine temporal stability in these signatures. Seasonal changes in environmental variables such as temperature and freshwater runoff may alter site-specific patterns through time, by influencing fish physiology (e.g. higher metabolic rates at higher temperatures) and bioavailability of some elements (e.g. Elsdon \& Gillanders 2006). Although not a strict limitation to the use of core signatures as markers of natal origin, temporal stability of some useful elements would greatly facilitate the practical application of this approach. Otherwise, atlases of natal signatures will have to be regenerated for each subsequent recruitment event.

\subsubsection{Optimal regional groupings by $S A$}

Nearby sites in the atlas have considerably different signatures (as evidenced from Fig. 4), and pooling these sites (without attention to this underlying heterogeneity) would result in a group with large variance and hence low overall classification success. In this case, the best groupings are not obvious from a MDS plot of site centroids (Figure 2.4). I thus aimed to optimize such clusters with respect to specific criteria, which can be modified to suit a given ecological question at hand: one could, for 


\subsection{Discussion}

example, leave out a location of interest, say a reserve or site of impact, and then optimally group remaining sites to optimize resolution with respect to desired predictive success.

While SA groupings did provide improvement for regional scale allocation success and optimized signal to noise ratio (Figure 2.3), even these optimal groupings did not achieve satisfactory allocation success at this scale. Nevertheless, this approach allows exploring the limits of resolution at a given scale and within a given dataset or for a given ecological question. For the modified cost function, for instance, the algorithm favoured small groups and thus a high resolution, which is generally synonymous with a loss in predictive power in the LDA. Yet the overall predictive success for the nine selected groupings was similar to that of the six a priori defined regions. The groupings thus seem to be more homogenous: no predictive power is lost by increasing the resolution with respect to a priori defined regions.

\subsubsection{Exclusion test}

For some applications, such as dispersal from and/or to protected populations whose viability might depend on external larval supply (Stobutzki 2001, Halpern et al. 2006), it may be of interest to estimate levels of self-recruitment versus external larval supply instead of explicitly identifying precise source locations. This may be achieved by using an exclusion test such as the one employed here. When considering a subset of locations from the total dataset, exclusion of non-local signatures is possible in certain settings where locations are sufficiently unique in their signatures. For Long Island, Island Bay and Kapiti sites, three marine reserves within the sampling domain, $95 \%, 85 \%$ and $76 \%$ of remaining signatures can be excluded respectively, thus providing a way to estimate larval supply to these marine reserves. If all other possible natal sites are sampled within the range of dispersal of the species, one may by 


\section{Chapter 2. Scale-dependent variability in hatchling otolith chemistry}

reciprocity also estimate larval supply from a marine reserve to surrounding localities by applying this test.

In this case, when I employed such a test on a large scale, I quickly became confronted with the problem of overlapping site distributions between regions: the proportion of individuals correctly rejected becomes very small as soon as I enlarge the focal area (location) to more than one site (e.g. multiple sites within a region). In such circumstances the application of an exclusion test would be misleading: failure to exclude a large proportion of 'foreign' fish from a larger subset of focal sites would result in an overestimate of self-recruitment. Standish et al. (2008) used a similar approach to ours for excluding recruits from a set of three potential source locations. However, no experimental tests were conducted on their dataset and it is difficult to know how well their exclusion test performed.

The repeated tests with truncated datasets further revealed how sampling effort is directly related to our ability to estimate the exclusion potential of the test. In my case, I can drop two sites from my dataset without dramatic changes in the percentage of correctly excluded individuals. If I drop ten sites, however, these estimates diverge by as much as $30 \%$, depending on which sites remained in the sample. For cases in which many sites with similar trace element signatures remained in the sample, estimates of correctly excluded individuals were poor relative to cases which included more heterogeneous sites. The likelihood of such extreme samples therefore depends on the number of sites sampled, and the extent of spatial covariance relative to the spacing of the sampled sites. To make any claims about the ability of an exclusion procedure to correctly exclude foreign signatures, the sample thus needs to encompass as much of the variability in signatures found across the dispersal range of the focal species as possible. Carefully considered sampling designs, e.g., informed by preliminary pilot studies, can be extremely useful in this respect, and 


\subsection{Discussion}

may lend increased confidence in the inferences derived from studies using otolith chemistry.

\subsubsection{Conclusion}

The aim with this chapter was to investigate spatial scales of variability in hatchling otolith signatures for this system, in the light of ecological questions involving larval dispersal. I demonstrated that site differences in an atlas of hatchling otolith signatures are exploitable at certain scales, the usefulness of which depends on the ecological question at hand. On some scales (regional scales in my system) supplementary information may help to improve the strength of inferences of dispersal pathways. The exclusion test provides a useful tool to assess larval subsidies from specific locations (e.g., marine reserves) on small scales. On a large scale, hatchling otolith microchemistry may yield insights into the question of present day dispersal between the main islands of New Zealand. In the light of such potential applications, further research into determinants of variability in hatchling otolith chemical signatures seems warranted, and could ultimately facilitate valuable insights into dispersal patterns in reef fish metapopulations. 


\section{Chapter 3:}

\section{Characterizing natal sources of fish using flexible Bayesian mixture models for otolith geochemistry}

\subsection{Introduction}

Geochemical tracers are increasingly used to reconstruct migrations and estimate demographic connectivity in both terrestrial and marine systems (Hobson 1999, Thorrold et al. 2002, Rubenstein \& Hobson 2004, Elsdon et al. 2008). Trace element tags contained within inert structures, such as fish otoliths, mollusc statoliths or bird feathers, have been used to quantify connectivity on ecological timescales (Swearer et al. 1999, Szép et al. 2003, Zacherl 2005), and stable isotopes have been employed to study migratory pathways of fish (Thorrold et al. 2001, Barnett-Johnson et al. 2008), birds (Rubenstein et al. 2002) and mammals (Burton \& Koch 1999).

Inferences from such geochemical tags generally involve initial sampling of individuals from populations of interest to establish a geographical baseline, or reference "atlas". Individuals of unknown origin are then assigned to one of the populations in this reference atlas based on their geochemical signature. This approach can illuminate dispersal patterns and the underlying mechanisms that govern dispersal for a species of interest by relating connectivity to environmental features (Schick et al. 


\subsection{Introduction}

2008, Shima \& Swearer 2009a, Swearer \& Shima 2010). Insight gained using geochemical tracers can thereby provide valuable insight about the interaction between dispersal and population level outcomes (Hamilton et al. 2008, Shima \& Swearer 2009a, 2010).

The identifiability of potential source sites or source populations is a major determinant of the success of such studies (Rubenstein \& Hobson 2004, Elsdon et al. 2008). In some coastal marine environments, the underlying environmental gradients can be gradual and/or populations may be poorly resolved by geochemical tags (Elsdon et al. 2008, Ruttenberg et al. 2008). Furthermore, such approaches generally requireor at least (implicitly) assume that all potential sources have been sampled in order to determine natal origins of recruits. Because comprehensive sampling is often not feasible, the utility of this approach may be limited in many marine environments (Campana et al. 2000, Munch \& Clarke 2008, White et al. 2008). Omission of potential source sites can limit the inferences one can make regarding dispersal in a given system. In particular, assignment of recruits to particular source sites may be erroneous if one does not have a complete atlas. This may be of lesser concern if there is spatial co-variation in signatures such that misassignments are made to spatially neighbouring sources (Pella \& Masuda 2006).

A solution to this problem has been proposed in genetics (Cornuet et al. 1999) and has recently been applied to otolith studies (Standish et al. 2008, Neubauer et al. 2010). The idea is to specify a criterion and to calculate the distribution (by simulation or analytically) of this criterion for the reference population. Based on this distribution, a threshold for belonging to this population can be established. If any fish of unknown origin falls outside of the, say, $95 \%$ confidence region of this distribution, the individual remains unassigned. One could thus potentially exclude 
individuals from populations that are not contained within the reference atlas. An empirical evaluation of this approach however showed that this method is only applicable on small scales in systems with a poorly resolved reference atlas and may be of limited use for large scale connectivity studies (Neubauer et al. 2010).

A different approach uses clustering methods to identify potential groups within a sample of recruits, which in turn may be related to likely geographical origins (White et al. 2008, Fontes et al. 2009, Shima \& Swearer 2009a). This alternative approach uses clustering of signatures followed by a form of model selection to estimate the most likely number of sources. If possible, the estimated clusters are then assigned to a given source or a collection of sources. However, such post-hoc interpretation of clustering patterns can be difficult, especially for clustering approaches which are not based on specific underlying models: it may not be clear what the observed clusters represent, i.e. to what spatial scale the cluster differences should be attributed, or whether clusters contain information other than spatial patterns (i.e. physiological/maternal effects on otolith chemistry). Most importantly, however, clustering methods do not use a baseline to separate groups, but rather just find the most homogenous groups within the individuals unknown origin. They are thus best suited for problems in which a baseline is completely absent. This is showcased by the classification results of the Bayesian clustering method presented in White et al. (2008): the clustering method with post-hoc assignment to sources often performs worse than linear discriminant analysis for simulated datasets.

Bayesian tools have gained considerable ground in the analysis of samples of mixed origin (i.e. stock mixtures in fisheries or recruits of unknown origin in connectivity studies), mostly due to the possibility to define realistic and probabilistically sound models which can incorporate 


\subsection{Introduction}

uncertainty at various levels of an analysis (Pella \& Masuda 2001, Manel et al. 2005, Pella \& Masuda 2006, Bolker et al. 2007, Munch \& Clarke 2008, White et al. 2008, Smith \& Campana 2010). As others have pointed out (i.e. Koljonen et al. 2005), most of these (as well as non-Bayesian methods) can be classified as either conditional or unconditional models. In conditional models, the baseline distributions (usually their mean and variance in geochemical studies), once estimated, are considered fixed and estimation of classification probabilities and mixing proportions are conditional on this estimate. In unconditional models, the baseline and mixture parameters are shared between the baseline and the mixed sample and are jointly estimated from both datasets. Koljonen et al. (2005) found that such methods usually perform better at mixed stock estimation tasks, due to the fact that the information about the baseline inherent in the mixed sample is used to better define their distributions. The models in this category can be further divided into many-to-one and many-to-many analyses (Bolker et al. 2007), depending on whether different mixed samples are considered independently or jointly across different locations of collection, respectively. This separation is meaningless in conditional models since the mixed stocks do not influence the source distributions.

Bayesian methods have been proposed to provide an answer to the problem of an unknown number of sources in a mixture. Pella \& Masuda (2001) proposed posterior predictive checks for an unconditional Bayesian mixture model from genetic characteristics to identify potential mismatches between the baseline and the mixed source sample. Smith \& Campana (2010) used these same checks for a joint model of otolith and genetic data. Such a mismatch could indicate the presence of un-sampled sources in the mixed sample. It does not, however, provide a way to estimate the nature and number of such extra-baseline populations. 


\section{Chapter 3. Bayesian mixture models for otolith geochemistry}

A Bayesian method to directly identify the contribution of extrabaseline sources to a mixture is provided by Pella \& Masuda (2006). Their use of a prior which has support over a theoretically infinite number of possible sources elegantly sidesteps the problem of model selection and provides a marginal distribution over the number of sources in the mixed sample. My focus in this chapter is to develop analogous models for geochemical data that follow the distribution assumptions commonly employed for this kind of data. I start by outlining Bayesian methods that extend parametric cluster analysis (White et al. 2008) by employing nonparametric Bayesian priors, and specifically Dirichlet Process priors (DP) (Escobar \& West 1995). Loosely speaking, non-parametric Bayes provides a flexible framework by formulating priors over model space, such as over models with different numbers of source populations. In this context, the DP model can be used to directly infer the number of possible natal sources in a mixture model if no baseline is available. I obtain marginal posterior distributions of the number of sources and marginal representations of relatedness of individuals in terms of their geochemical signatures. This framework is then extended to include baseline samples in an unconditional analysis which classifies fish with either the baseline samples or out-of-sample classes. In an extension of the method proposed by Pella \& Masuda (2006), I describe different ways in which a mixed sample can be clustered with such a baseline. Lastly, I propose a new method for hierarchical unconditional classification, which can be used with an incomplete reference atlas. This model employs a classification procedure at large scales and an embedded non-parametric clustering at smaller scales. These models are tested on the well-known weakfish data set (Thorrold et al. 2001), which has been previously evaluated by other authors to illustrate Bayesian methods for otolith data (Munch \& Clarke 2008, White et al. 2008). 


\subsection{Statistical models}

\subsection{Statistical models}

\subsubsection{A Dirichlet process mixture (DPM) model for clustering}

In this section I introduce the DPM, an extension of the finite mixture model commonly employed in mixed sample analyses. The goal of this model is similar to that of White et al. (2008) and the model has many of the same limitations, but an analytical integration step makes it possible to circumvent the problem of model selection in mixture models and to infer the number of potential sources directly.

I assume that the p-dimensional vector of geochemistry data for individuals $i=(1 \ldots N)$, denoted $y_{i}=\left(y_{i, 1}, \ldots, y_{i, p}\right)$, comes from an unknown underlying multivariate normal distribution $N\left(\theta_{i}\right)$, where the set $\theta_{i}=\left\{\mu_{i}, \Sigma_{i}^{2}\right\}$ includes the mean vector and covariance matrix for the distribution of $y_{i}$. Furthermore, the $\theta_{i}$ are drawn from a mixing distribution $G$, itself drawn from a Dirichlet Process, a distribution over individual source distributions in the mixed sample. The hierarchical version of this model can be written as (Neal 2000):

$$
\begin{aligned}
y_{i} \mid \theta_{i} & \sim N\left(\theta_{i}\right) \\
\theta_{i} \mid G & \sim G \\
G & \sim D P\left(\gamma, G_{0}\right)
\end{aligned}
$$

The Dirichlet Process is parameterized by $G_{0}$ and $\gamma . G_{0}$ is called the base measure for the source specific multivariate normal distributions: it is the prior expectation of $G$ such that $E[G(\theta)]=G_{0}(\theta)$. The $\gamma$ parameter measures how centred source distribution parameters are around the base distribution $G_{0}$, as demonstrated below, and determines the number of sources in the model. A small $\gamma$ is synonymous with few sources and viceversa. The DPM can be derived in a number of ways, leading to equivalent distributions over partitions of the data. The most appropriate in the context of the mixed sample problem in fisheries and ecology is a 
derivation of the DPM as the infinite limit of the finite mixture model (Neal 1992, Neal 2000). In this view we have a theoretically infinite number of sources that could contribute to a sample. In practice only a limited number of sources will contribute to the mixed sample, as detailed below.

The most important feature of this model is the discreteness of the draws from a $D P$ : values drawn from the $D P$ will have positive probability to coincide. In other words, some individuals will share a common parameter vector $\theta$, indicating that they originate from the same source. This is the basis for clustering in the model. To see this, consider the prior for $\theta$ that arises if $G$ is integrated out of the model. This can be shown to be a series of draws from the conditional distribution of $\theta_{i}$ given all other $\theta^{-i}$ (Ferguson 1973):

$$
\theta_{i} \mid \theta^{-i}, \gamma \sim \frac{1}{N-1+\gamma} \sum_{j \neq i}^{N} \delta\left(\theta_{j}\right)+\frac{\gamma}{N-1+\gamma} G_{0}
$$

where $\delta\left(\theta_{j}\right)$ is a point mass at $\theta_{j}$. This is a mixture formulation: with probability proportional to $\gamma$ a new instance of $\theta$ will be drawn from $G_{0}$, else $\theta_{i}$ will coincide with one of the $j$ previous instances of $\theta$. From this formulation it is clear that the number of sources drawn in this model will be finite in practice.

This conditional formulation of the $D P$ prior is very useful in another way: it makes drawing samples form the posterior distribution of the DPM model given the data $Y$ rather straightforward. Note that (3.2) is only the conditional prior distribution of $\theta_{i}$ and that Bayes theorem will combine it with the likelihood $P\left(y_{i} \mid \theta_{i}\right)$ to give the posterior distribution of the source parameters $\theta_{i}$. The source parameters $\theta_{i}$ do not need to be sampled explicitly; it is often easier to obtain samples from source assignments $c_{i}$ to source $k$ (though they are readily obtainable from the conjugate construction employed below if needed). This is so because the 


\subsection{Statistical models}

$\theta_{i}$ which coincide define a source membership, and the actual source parameters can be integrated out of the model, as in Munch \& Clark (2008). This integration makes Gibbs sampling more effective as it limits the number of states of the sampler. The conditional prior given above can then be written in terms of individual source assignments:

$$
p\left(c_{i}=k \mid c^{-i}, \gamma\right)= \begin{cases}\frac{n_{k}^{-i}}{n-1+\gamma}, & k \leq K_{+} \\ \frac{\gamma}{n-1+\gamma}, & k>K_{+}\end{cases}
$$

where $K^{+}$is the number of sources currently associated with an observation and $n_{k}^{-i}$ is the number of individuals associated with source $k$ except individual $i$. The prior is similar in interpretation to the classical mixture proportion in finite mixture models: the larger the proportion $n / N$ of individuals assigned to a given source, the larger the prior probability that the next individual considered will come from this source as well. To sample from the full posterior distribution one can alternate Gibbs sampling of source assignments and sampling of $\gamma$, which is directly analogous to sampling in finite mixtures (see for instance the Gibbs sampler in Munch \& Clarke 2008). To sample source identities, the prior for the i-th observation is combined with its likelihood and the following probabilities can be used to draw samples of assignments $c=c_{1} \ldots c_{i} \ldots c_{n}$, (Neal 2000):

$p\left(c_{i}=k \mid c^{-i}, \gamma, Y\right)=\alpha \begin{cases}\frac{n_{k}^{-i}}{n-1+\gamma} \int N\left(y_{i} \mid \theta_{k}\right) d H_{-i}\left(\theta_{k}\right), & k \leq K_{+} \\ \frac{\gamma}{n-1+\gamma} \int N\left(y_{i} \mid \theta_{k}\right) d G_{0}\left(\theta_{k}\right), & k>K_{+}\end{cases}$

where $H_{-i}\left(\theta_{k}\right)$ is the posterior distribution of $\theta_{k}$ given data $y_{c_{k}^{-i}}$, is the data of all fish associated with source $k$ except the data of fish $i . \alpha$ is a constant assuring that this probability integrates to 1 . I use a vague multivariateNormal-inverse-Wishart conjugate prior for $G_{0}$, which makes it possible to solve the integration over $\theta$ analytically. This prior has four parameters, the prior mean $\mu_{0}$ and scale matrix $\Delta_{0}$ as well as the prior degrees of freedom 


\section{Chapter 3. Bayesian mixture models for otolith geochemistry}

for the scale matrix $\left(\nu_{0}\right)$ and a prior parameter $\kappa_{0}$ which relates the variance of $\mu_{k}$ to $\Sigma_{k} \cdot \int F\left(y_{i} \mid \theta_{k}\right) d H_{-i}\left(\theta_{k}\right)$ is then a multivariate student-t density for source assignments with parameters updated according to the observed data (Gelman et al. 2003):

$$
\begin{aligned}
y_{i} \mid y_{c_{k}^{-i}}, G_{0} & \sim t_{\nu_{n}-D+1}\left(\mu_{n}, \Delta_{n}\left(\kappa_{n}+1\right) / \kappa_{n}\left(\nu_{n}+D+1\right)\right) \\
\mu_{n} & =\frac{k_{0}}{k_{0}+N} \mu_{0}+\frac{n_{c_{k}}^{-i}}{k_{0}+n_{c_{k}}^{-i}} \bar{y}_{c_{k}^{-i}} \\
\kappa_{n} & =\kappa_{0}+n_{c_{k}}^{-i} \\
\nu_{n} & =\nu_{0}+n_{c_{k}}^{-i} \\
\Delta_{n} & =\Delta_{0}+S S_{c_{k}^{-i}}+\frac{\kappa_{0} n_{c_{k}}^{-i}}{\kappa_{0}+n_{c_{k}}^{-i}}\left(\bar{y}_{c_{k}^{-i}}-\mu_{0}\right)\left(\bar{y}_{c_{k}^{-i}}-\mu_{0}\right)^{T}
\end{aligned}
$$

The subscript 0 denotes the prior value for the parameter, and SS is the sum of squares within the considered source. The second case in (3.3) defines a new source, the $\theta_{k}$ is therefore defined by the observation $y_{i}$ alone, and $K^{+}$is augmented by 1 if this case is drawn. Integrating with respect to the prior $G_{0}$ gives the same predictive likelihood as before with $y_{i}$ as the sole observation of the new class, and the prior parameters determine the predictive likelihood in (3.4). Finally, $\gamma$ is estimated from data using Gibbs sampling procedures described in Escobar \& West (1995), using a vague Gamma $(1,1)$ distribution as prior. The conditional posterior is then a mixture of Gamma distributions:

$$
\gamma \mid \xi, k \sim \pi_{\xi} G(1+k, 1-\log (\xi))+\left(1-\pi_{\xi}\right) G(1+k-1,1-\log (\xi))
$$

where $\xi$ follows a beta distribution such that $\xi \mid \gamma, k \sim B(\gamma+1, N)$ and

$$
\pi_{\xi}=\left(K^{+}-K^{+} \log (\xi)\right) /\left(K^{+}-K^{+} \log (\xi)+N\right)
$$

Posterior estimation can be difficult in the DPM when the posterior is multimodal, which is the case with any mixture model estimated with latent (unknown) classes. Since the labels of assignments $c$ are irrelevant to the actual estimation, the model is said to be invariant with respect to 


\subsection{Statistical models}

the class labels. One would thus obtain the same partition into clusters with every combination of class labels, such that for $K$ sources there would be $K$ ! different posterior modes. The Gibbs sampler has a tendency to remain in one of these modes and often does not explore the posterior distribution properly. This occurs because the steps necessary to switch between class labels have very low probability of occurring. Only using the Gibbs sampling described above may therefore not guarantee adequate sampling from that the posterior distribution (Jain \& Neal 2004). To ensure adequate mixing of the sampler I coupled the Gibbs sampler over only $\mathrm{C}$ and $\gamma$ with a split-merge sampling step (Jain \& Neal 2004). A split merge step helps the sampler pass regions of low posterior probability in parameter space, thereby improving sampling from the full posterior. This is achieved by splitting or merging clusters formed by Gibbs sampling, where splits or merges are accepted with a Metropolis-Hastings acceptance probability to ensure convergence to the stationary distribution (Jain \& Neal 2004). Specifically, I used the improved splitmerge procedure with sequential allocation (Dahl 2003) to achieve optimal performance of the sampling scheme. For a detailed discussion of this sampler in the context of mixed stocks from a genetic perspective, see Pella \& Masuda (2006). Changing the likelihood to that corresponding to (4) gives a direct correspondence of their sampling scheme with the one used for this study. All inferences presented in this paper are based on 10000 samples from the posterior distribution of the model after a burn-in of 1000 samples was discarded. Convergence of the Markov chains was checked visually and confirmed from multiple starts with random initial conditions.

\subsubsection{Using the DPM with a baseline}

The above stated DPM model can be applied as such, without a baseline. It is however straightforward to appreciate why this is difficult and of limited use with otolith chemistry: the number of sources selected 
by the model will depend on the prior for the covariance matrix. If the prior is vague, the covariance matrix needs to be estimated from data, which may be difficult when the data are not clearly separated. As a result it will be difficult to know to which spatial scale the estimated number of sources pertains. To extend the use of the DPM, I propose two separate ways to use this model with a baseline: 1) in a clustering and 2) in a classification (or discrimination) context. The latter has been discussed by Pella and Masuda (2006) with genetic data. It consists of applying the DPM to the mixed sample with a certain number of fixed populations. Sampled individuals are assigned to either an existing source or an extra-baseline source in the Gibbs sampler. The probability that this individual comes from one of the proposed sources or from an un-sampled baseline source can then be estimated. Incorporation of the baseline into the sampling scheme described above is straightforward and the necessary additions are detailed by Pella and Masuda (2006).

In some applications however, we may know (e.g. from crossvalidation studies) that assignment success to one of the baseline populations will be rather low. This is often due to limited resolution in the dataset, but as illustrated elsewhere (Smouse et al. 1982, Neubauer et al. 2010), a large number of baseline sources can make assignment difficult even with good local resolution - all else being equal, assignment success will generally decrease with an increasing number of sources considered. Furthermore, using otolith chemistry to define a source population may lead to multimodal distributions if variation occurs on scales smaller than the one on which a source is defined (see discussion). In some instances we may thus have little confidence in the actual assignments to the baseline sources if these are highly resolved (i.e. individual embayments or islands) (Warner et al. 2005, Ruttenberg \& Warner 2006, Ruttenberg et al. 2008, Neubauer et al. 2010). I propose that in such instances a DPM clustering of the mixed sample (or recruits) of unknown origin with the baseline as a 


\subsection{Statistical models}

joint dataset may provide more insight. For some regions in parameter space some individual sources may be rather well defined and some clusters will therefore consist of individuals from a single source, adjacent sources, or perhaps a regional source pool (see, for instance, White et al. 2008). Mixed samples which are clustered within such pure clusters can therefore be regarded as more certain classifications, samples within mixed clusters will represent uncertain classifications. This feature is illustrated below on the weakfish dataset.

3.2.3 Extension to hierarchical classification with an incomplete atlas

The DPM essentially performs a model-based clustering where the number of clusters is a marginal quantity of the posterior distribution of the model. Basing the clustering on such a model can provide distinct advantages, the most relevant of which is that the number of sources does not need to be specified, nor do we need to define a criterion to find the most likely number of such sources. Here, I take this approach a step further and embed the DPM clustering model detailed above in a classification model. I propose to perform classification on a continuous map, for instance at the scale of regions within the total sampled area, where each region is modelled by a Dirichlet Process mixture of its individually sampled sources (Figure 3.1a). In other words, I replace the commonly used normal density in a (Bayesian) mixture or discriminant analysis for otolith chemistry (or other continuous data) by a mixture density.

Formally, classification within a discriminant analysis or mixture model involves the use of Bayes theorem to calculate posterior assignment probabilities

$$
p\left(\zeta_{i}=q \mid y_{i}\right)=\frac{f\left(y_{i} \mid \theta_{q}\right) \pi_{q}}{\sum_{q^{\prime}=1}^{Q} f\left(y_{i} \mid \theta_{q^{\prime}}\right) \pi_{q^{\prime}}}
$$




\section{Chapter 3. Bayesian mixture models for otolith geochemistry}

where $\zeta_{i}$ assigns a source $q$ to fish $i$, based on the likelihood $f\left(y_{i} \mid \theta_{q}\right)$ of fish $i$ given the source specific parameters $\theta_{q}$ and a specified prior $\pi_{q}$ (amounting to a discriminant analysis) or estimated mixture proportion $\pi_{q}$ (the mixture model setup) for sources in the baseline. The normal density is often used to define the likelihood for such a classification with continuous data, with $\theta_{q}$ estimated from baseline data (Munch \& Clarke 2008, White et al. 2008). The central limit theorem assures that the sum of independently drawn random variables is normal and thus even if differences exist on scales smaller than the one on which we define a source, the data will be asymptotically normal if a large number of fish from each source are sampled. Since this result holds only asymptotically however, we may in practice see multimodal distributions at the randomly defined scales at which regions or sources are delimited. For instance, a source may be defined over a stretch of coast or a spawning site which receives local freshwater input or experiences a localized upwelling. One may then expect some elements (i.e. Barium) to be significantly different between individuals that experienced such an anomaly within the source, and the distribution would be bi-modal at the scale of the source. The normal distribution may in that case be a poor approximation of the true density of each source.

The main idea behind the classification model proposed here is to subdivide the entire region of study (the region that may contribute to local recruit pools) into contiguous but adjacent regions (see Figure 3.1 for an illustration of this method), each of which is modelled by a DPM. This could be especially useful for hierarchically structured datasets (i.e. Ruttenberg \& Warner 2006, Miller 2007b, Ruttenberg et al. 2008, Neubauer et al. 2010). If each region has been sampled representatively, it may then be possible to estimate contributions from these $Q$ regions to a recruit pool. This reasoning applies to any classification procedure. Indeed, even a single sampling site is an arbitrarily scaled region, and problems 


\subsection{Statistical models}

arise because at this scale these regions are not adjacent: not all potential source regions are sampled, and classification may become meaningless. In many cases it will thus be essential for the classification procedure detailed here to define these regions on a continuous spatial scale (though see the weakfish application below).

The classification will force a large-scale structure at the regional scale, while retaining the flexibility of the DPM cluster model at smaller scales. It is hoped that this sub-division of the study domain into regions mitigates the problem of a poorly resolved atlas by classifying recruits to a region within which sites may be more readily identified. Furthermore, the model can be specified for a fixed baseline (and thus have fixed baseline sites within each region), or the mixture and baseline can be clustered together within regions. The nature of the DPM allows for the possibility that a sampled recruit belongs to a non-sampled entity within any of the regions. Formally,

$$
f\left(\boldsymbol{x}_{\boldsymbol{q}} \mid \theta_{q}\right)=D\left(F, G_{q}, \gamma\right)
$$

where $D$ is the mixture density of the DPM model and $F$ specifies the source density within regions. In other words, I assume that the data $\boldsymbol{x}_{q}$ from each distinct region $q$ in the atlas is a mixture of an unknown number of (normal) distributions within each region. The essential part of the classification is a hierarchical prior on $G_{q}$, which will define the region specific attribute and will thus determine if a fish that does not fit well with any of the sampled sources is likely to have come from an unsampled source within that region. 


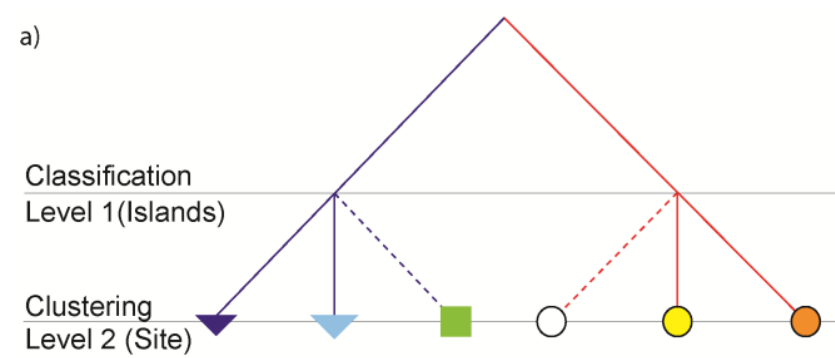

b)

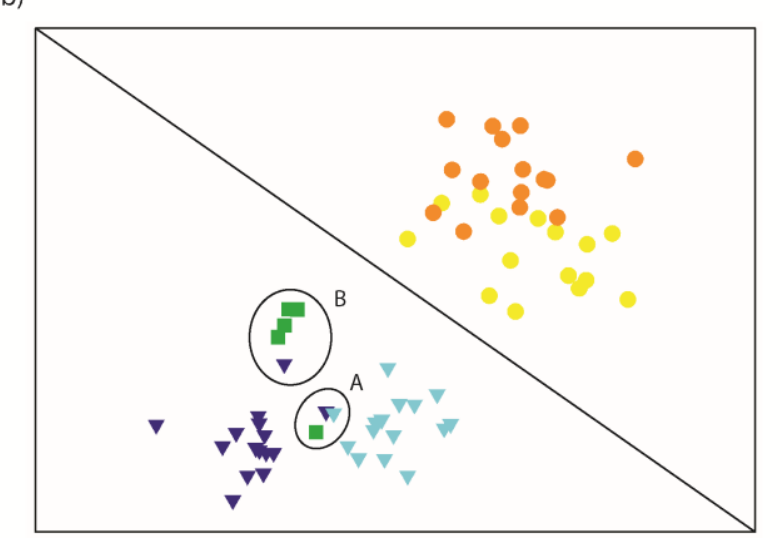

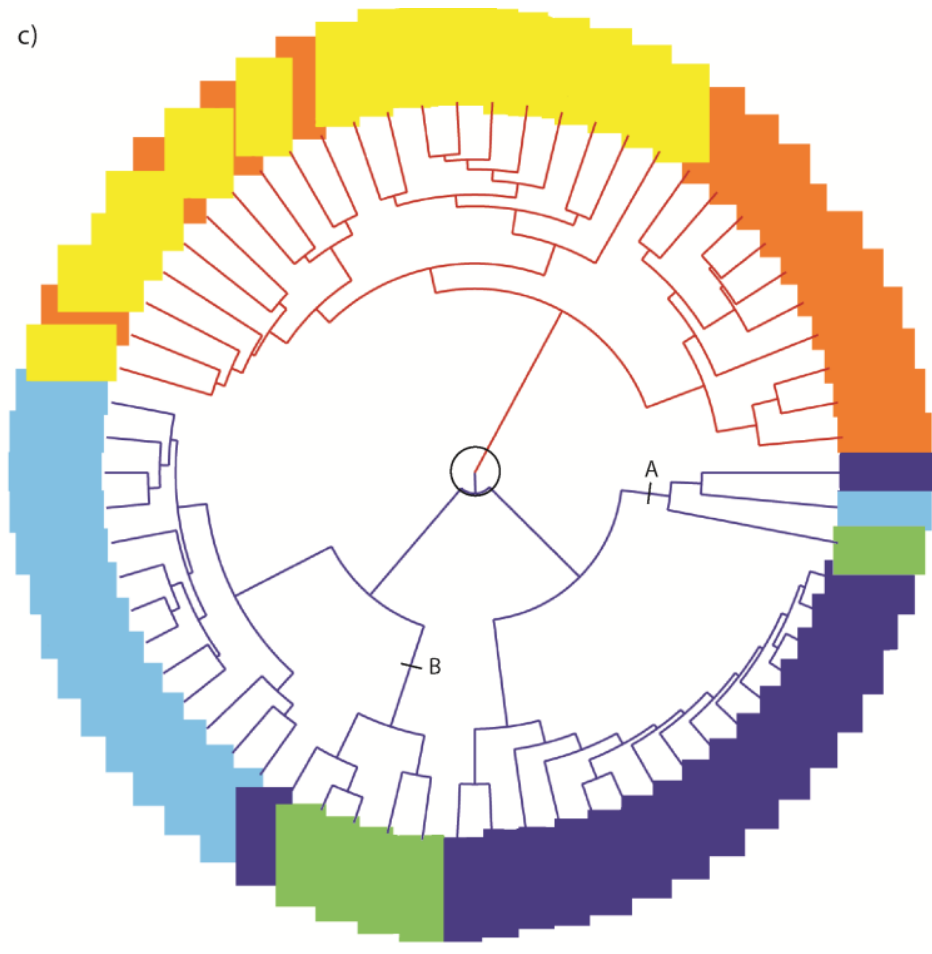

Figure 3.1: Illustration of a) the DPMc classification procedure (with a non-fixed baseline for the DPM), b) simulated data and c) DPMc results graphed as a marginal tree. a) The classification scheme works by first dividing the domain up into regional entities (i.e. Level $1=$ Islands). Within these entities, the DPM models the atlas, and each recruit is clustered with either existing groups within the atlas or defines a new group (i.e. Level $2=$ Sites within Islands). A tree can be constructed which includes atlas signatures and recruits. Dashed branches illustrate the fact that these groups are not existent in the atlas but may come into existence when mixed sample is collected: in the example some fish come from a site which is not present in the simulated atlas (green). b) Simulated baseline data are in two main regional groups (corresponding symbols, triangles and circles, on either side of $x=-y$ ) and subdivided into sites i.e. sites, corresponding to leaf colours. Green squares indicate the extra baseline source. Corresponding cluster A and B are indicated in c), the resulting marginal tree which illustrates the relationships in the data (see section 3.2.4): node heights are equal to co-assignment probabilities of individual (or sets of) fish to clusters (i.e. sites) within regions. The black circle toward the centre represents a co-assignment probability of 0.05 , co-assignment probabilities are increasing toward the leaves (where they equal 1). 


\subsection{Statistical models}

Specifically, for Bayesian classification based on the reference atlas, the posterior predictive likelihood $f$ of the new observation given the baseline is needed (Munch \& Clarke 2008): for baseline data $x_{q}$ from regions $q=1 . . Q$, this is the likelihood of observing $y_{i}$ given the posterior distribution of parameters estimated from $x_{q}$ (and $y^{-i}$ for unconditional classification). The posterior density of the DPM model can be summarized by draws of the parameters $\theta_{q}$, the set of source parameters in region $q$, if these are not integrated out. The posterior predictive distribution for $y_{i}$ is then defined as (Escobar \& West 1995, Munch \& Clarke 2008)

$$
f\left(y_{i} \mid \zeta_{i}=q, x_{q}\right)=\int f\left(y_{i} \mid \zeta_{i}=q, \theta_{q}\right) p\left(\theta_{q} \mid x_{q},\left(y^{-i}\right)\right) d \theta_{q}
$$

This density is complex and the integral is not analytically solvable. A similar MCMC as for the above clustering model can, however, be used to evaluate this density for each region $q$ and assign recruits to the region of highest posterior density. The predictive distribution can therefore be approximated by Monte Carlo (Neal 2000):

$$
E\left(f\left(y_{i} \mid \zeta_{i}=q, x_{q},\left(y^{-i}\right)\right) \approx \frac{1}{T} \sum_{t=1}^{S} f\left(y_{i} \mid \zeta_{i}=q, \theta_{t, q}\right)\right.
$$

Dropping the dependence on Monte Carlo draws $t$ for sake of generality, it follows from (3.2) that $f\left(y_{i} \mid \zeta_{i}=q, \theta_{q}\right)$ can be evaluated as $f\left(y_{i} \mid \theta_{i}\right)$ if $\theta_{i}=\theta_{j}$ for some $j \neq i$ or as $\int f\left(y_{i} \mid \theta_{i}\right) d G_{q}\left(\theta_{i}\right)$ if $\theta_{i} \neq \theta_{j}$ for all

$j \neq i$. The configuration of sources from the atlas is thus used to calculate the predictive likelihood for each fish in the mixed sample to come from one of the sources within region $q$, allocating it to either a sampled or an un-sampled source within region $q$. Keeping the predictive likelihood for each region $q$, one can perform classification using Bayes theorem. The algorithm which performs this classification can be described as follows: 
For $t=1 \ldots T$ steps of the MCMC do:

For each individual $i$,

a. Draw

$$
p\left(c_{i, t, q}=k_{q} \mid \theta_{t, q}, \gamma_{t, q}\right)=\alpha \begin{cases}\frac{n_{t, k_{q}}^{-i}}{n_{t, q}-1+\gamma_{t, q}} F\left(y_{i} \mid \theta_{t, k_{q}}\right), & k_{q} \leq K_{q} \\ \frac{\gamma_{t, q}}{n_{t, q}-1+\gamma_{t, q}} \int F\left(y_{i} \mid \theta_{t, k_{q}}\right) d G_{q}\left(\theta_{t, k_{q}}\right), & k_{q}>K_{q}\end{cases}
$$

in each region $q . n_{t, q}$ is the number of fish in the mixture currently assigned to region $\mathrm{q}$ and $n_{t, k_{q}}^{-i}$ is the number of fish assigned to source $k_{q}$ - this count excludes individual $i$ if individual $i$ is currently associated with $k_{q}$. These counts also exclude baseline cases.

b. Save the predictive likelihood $f\left(y_{i} \mid \zeta_{i}=q, \theta_{t, i}\right)$.

c. Calculate the posterior probability that fish i comes from region $q$

$$
p\left(\zeta_{t, i}=q \mid \theta_{t}, y_{i}\right)=\frac{f\left(y_{i} \mid \zeta_{i}=q, \theta_{t, k_{q}}\right) \pi_{t, q}}{\sum_{q^{\prime}=1}^{Q} f\left(y_{i} \mid \zeta_{i}=q^{\prime}, \theta_{t, k_{q^{\prime}}}\right) \pi_{t, q^{\prime}}}
$$

For unconditional classification, update $\theta_{q}$ of the sources in each region $q$ based on $\zeta_{t, i}$, the individuals of the mixed sample assigned to each region. Else skip this step for conditional classification.

Update $\pi_{q}$ given the assignments by drawing from $\pi_{q} \mid n_{q} \sim \operatorname{Dirichlet}\left(1 / Q+n_{q}\right)$, where $n_{q}$ is the number of individuals currently assigned to region $q$.

Update $G_{q}$ and $\gamma_{q}$ in all regions given the assignments 


\subsection{Statistical models}

\subsubsection{Marginal descriptions of source assignments}

Mixture models are prone to label switching, a phenomenon which occurs during iterative estimation routines of latent (unobserved) class models. The actual labels of these classes are arbitrary and switch throughout the estimation routine. Unless one adopts a restrictive formulation, DPMs also exhibit this property. To deal with this problem, a re-labelling algorithm to recover the most probable labels can be used (e.g. White et al. 2008). Such procedures are not without pitfalls (see for instance Dawson \& Belkhir 2009 for available approaches for dealing with the label switching issue and a discussion of these).

I follow Dawson \& Belkhir (2009) and circumvent this problem by noting that the sources are only defined by the observations (or specifically, individual fish) that constitute them. Marginal posterior coassignment probabilities of individuals that are invariant to label switching can be obtained to express the degree of their similarity (Huelsenbeck \& Andolfatto 2007, Dawson \& Belkhir 2009). This quantity expresses the probability that two individuals (or a set thereof) are assigned to the same cluster (or source in this case), given the uncertainty about the number and nature of these clusters. It is therefore a marginal probability: it integrates over the posterior distribution of model parameters, and thereby takes into account the uncertainty inherent in this distribution.

To visualize patterns in the collected recruits from each site, I applied the exact linkage algorithm of Dawson \& Belkir (2009), which constructs a tree (akin to a hierarchical clustering tree) based on estimates of marginal co-assignment probabilities. These probabilities are the probability that two fish or sets of fish come from the same source given all uncertainty inherent in model parameters. The node height of any node in the constructed tree is the estimated posterior co-assignment probability of the sets which are merged by that node. One can then look for marginally 
significant groups: these are clusters in the tree which are co-assigned with probabilities smaller than some threshold, say $p_{c}=0.05$. Since coassignment probabilities integrate over uncertainty in the model parameters and number of sources, these marginal co-assignment probabilities can be regarded as minimal co-assignment probabilities. We can therefore expect only well resolved sources to be significantly different at this level.

I use a similar logic in dealing with output from the classification procedure. Here, I aim to construct a tree that gives co-assignment probabilities given not only the uncertainty about sources within each region, but which further marginalizes over classification probabilities to these regions. For this, I cluster the mixed sample with atlas signatures of the region which recruits were assigned to at each iteration of the MCMC algorithm. This procedure marginalizes over classification assignment probabilities and the exact linkage algorithm can be applied to construct a tree. This can provide substantial information in the case of an incomplete atlas. An illustration of this is given in the simulated classification example (Figure 3.1c). Tree plotting and annotation was performed using TreeDyn (Chevenet et al. 2006).

\subsection{Application}

\subsubsection{DPM clustering models}

To illustrate the DPM clustering method, I tested models with and without baseline on the weakfish dataset of Thorrold et al. (2001). The premise of the original study was to investigate philopatry in weakfish using otolith core signatures of adult weakfish which were compared to a baseline of otolith chemistries collected from juvenile weakfish two years earlier. The authors use a discriminant analysis to assign adults to natal estuaries, thus assuming that i) adult fish were spawned in one of five 


\subsection{Application}

baseline estuaries and ii) that these estuaries were sufficiently characterized by the data collected from juvenile fish to allow for such a classification.

There are a number of motivations for using DPM models for a reanalysis of this dataset. i) Recent models developed for assigning natal origins (or estimating source proportions) based on otolith chemistry, have used this dataset for illustrative purposes (Munch \& Clarke 2008, White et al. 2008). ii) While the original study notes that the five estuaries under investigation account for $90 \%$ of commercial weakfish catch, other estuaries along the east coast could have potentially accounted for some of the adult fish in this study. iii) Some of these estuaries are relatively large (i.e. Chesapeake Bay is the largest estuary in the USA), and large variation in otolith chemistry can often be found even on small scales within estuaries (Miller 2007a). This could lead to multi-modal distributions within estuaries and the normal distributions used in previously applied models may be a poor fit.

Thorrold et al. (2001) use chemistry measured from whole otoliths for the juvenile baseline, whereas chemical measures on adult fish otoliths were from the core only. I found that values for Magnesium were higher within cores of adult otoliths than in whole juvenile otoliths. Recent studies suggest that many elements are elevated in the otolith core of many species (i.e. Mg, Mn, Ba (Brophy et al. 2004, Ruttenberg et al. 2005)). An ontogenetic signal and/or a difference in sampling methods for juvenile fish may therefore have led to lower $\mathrm{Mg}$ values in the reference atlas. $\mathrm{Mg}$ was hence excluded from the analysis (to little overall effect).

\subsubsection{Clustering fish without a baseline}

I started by investigating whether the DPM model without a baseline would successfully separate the baseline (juvenile fish from known locations) into the five estuaries that were sampled. For this I used a vague 
prior of $\gamma \sim \operatorname{Gamma}(1,1), \nu_{0}=p, \kappa_{0}=1, \mu_{0} \sim N(0, \eta), \Delta_{0}=\operatorname{cov}(X)$ and $\eta \rightarrow+\infty . \mu_{0}$ is thus not fixed but estimated with a flat prior, using methods described in Escobar \& West (1995). $p$ is the number of elements considered in the analysis. The prior guess at the covariance matrix is (naively) the covariance matrix of the whole dataset. Since $\nu_{0}$ is small relative to $\mathrm{N}$, the prior influence should be minor unless clusters with very few individuals exist. To further illustrate some difficulties associated with the use of the DPM for simple clustering based on geochemical signatures, I simulated datasets of varying degrees of separation between sources as summarized by Table 3.1 .

Table 3.1: Posterior expectation and mode for the number of sources in each of four simulation experiments. The variance of the marginal posterior distribution over sources are given for reference. Simulations included either three or five sources and high or low separation, as measured by the generalized (or Mahalanobis) distance. These simulations were designed to emulate generalized distances between classes found in either the weakfish dataset (high separation distance $d=32.35$ ) or the otolith core dataset of Neubauer et al. 2010 (low separation between sites: $d=11.85$ ).

\begin{tabular}{|c|c|c|}
\hline \multirow{2}{*}{ Scenario } & \multicolumn{2}{|c|}{$E\left(K^{+}\right) \|$mode $\left(K^{+}\right)$} \\
\cline { 2 - 3 } & 3 sources & 5 sources \\
\hline \hline High & $3.09 \| 3$ & $3.73 \| 4$ \\
$\mathrm{~d}=32.96$ & 1.18 & 1.47 \\
\hline Low & $2.61 \| 2$ & $3.32 \| 3$ \\
$\mathrm{~d}=12.33$ & 1.72 & 1.98 \\
\hline
\end{tabular}




\subsection{Application}

Analysis of simulated data showed that even with high overall separation in the dataset the model does not necessarily recover the correct number of sources (as indicated by the posterior mode) (Table 3.1), though the correct number is generally within the $95 \%$ credible interval. The performance naturally deteriorates when separation between sources is lower. In fact, the distribution over sources depends solely on the configuration of sources in multivariate space. If only two sources are very similar, the correct number of sources will likely not be recovered. Individual pairwise generalized distances in the simulation with high separation ranged from $10.12-81.48$, as compared to $6.06-83.21$ in the original weakfish dataset. Surprisingly, when applying the DPM to the weakfish baseline, I find that the number of sources estimated from the model are too high rather than too low $\left(E\left(K^{+}\right)=7.63\right.$, Figure 3.2). Some multi-modality is thus evident at the baseline level.

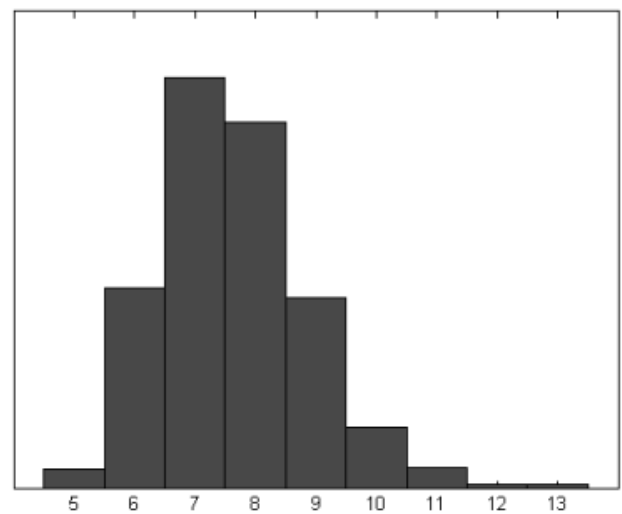

Figure 3.2: Histogram of the marginal posterior distribution over the number of sources in the weakfish baseline dataset. 


\section{Chapter 3. Bayesian mixture models for otolith geochemistry}

\subsubsection{Clustering fish with a baseline}

To test how the DPM models perform with inclusion of a baseline I conducted a series of test scenarios. First, I used a self-assignment test, assigning juveniles of known locations to a baseline (or extra baseline sources) estimated from these same individuals. Such a self-assignment test is obviously biased towards inflating correct assignments, and will serve here solely to validate the method. If the model performs well, individual juveniles should i) not be attributed to extra-baseline sources where there shouldn't be any (since all sources in the self-assignment tests are known), and ii) be assigned to the correct source.

The self-assignment test provided interesting insight into the structure of the dataset, the workings of the DPM method, as well as some explanations for previous results with this same dataset. In particular, the posterior mode of the number of sources in the dataset is at six sources, however the tree produced by the exact linkage algorithm clearly shows that the sixth source consists of only four individuals (Figure 3.3), three of which are from the closest tree - that of samples including the Delaware (DE) baseline. It is thus likely just an artefact of the dataset and specifically of the multimodality in the DE baseline. Indeed DE samples also often cluster with those from New York, highlighting the multi-modality for this source. Furthermore, misclassification was most often to geographically neighbouring estuaries for all sources. Munch and Clark (2008), noting straying of adult fish to neighbouring estuaries (as originally found by Thorrold et al. 2001), indicated that geographical straying patterns are not discernible from misclassification. The self-assignment test however indicates that such evidence of straying may indeed be due to misclassification to neighbouring estuaries, and that natal homing may be higher than estimates would indicate. Overall classification success (86.5\%) was in line with that reported from LDA (>85\% correctly assigned). 


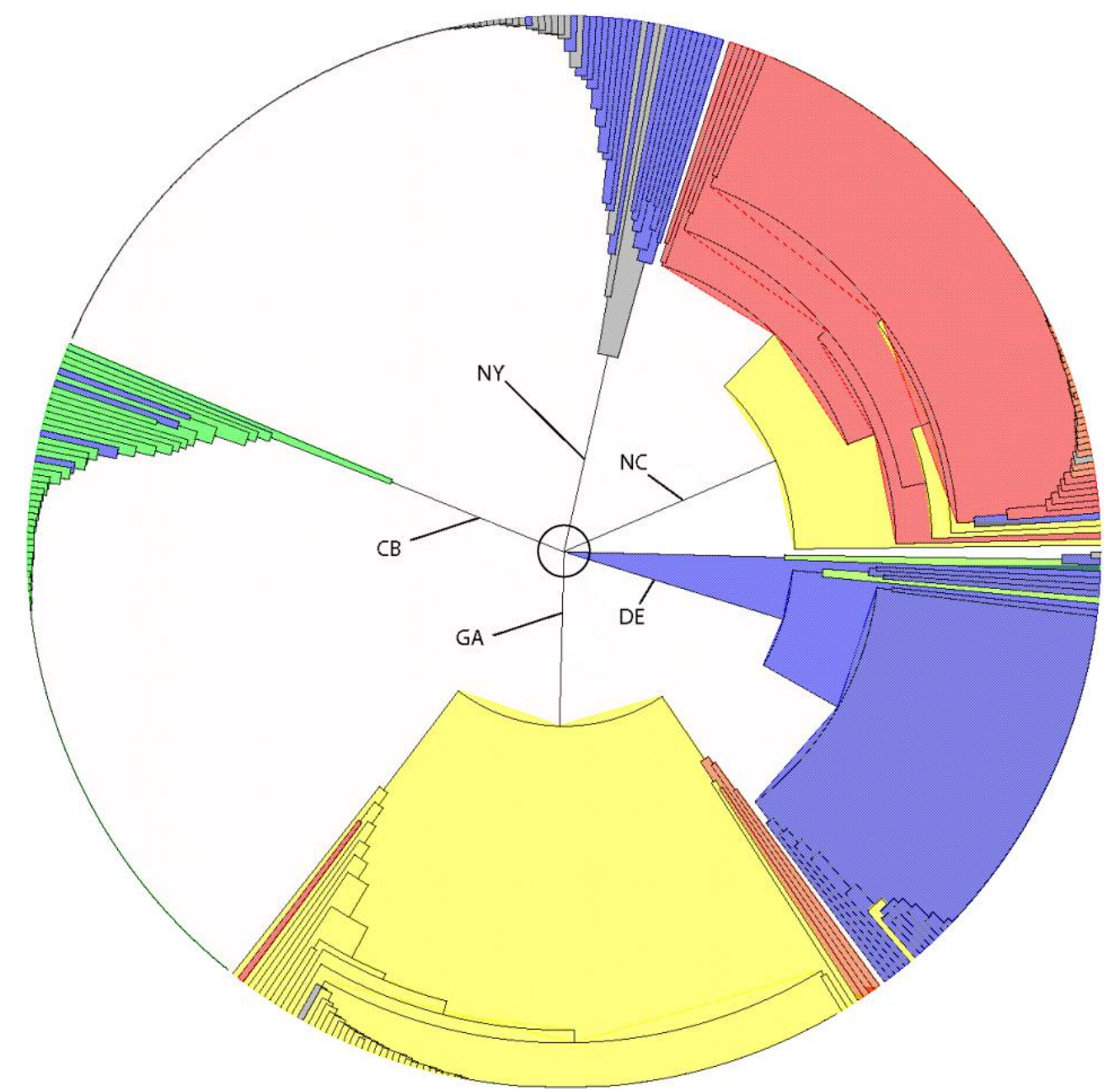

Figure 3.3: Exact linkage tree from a self-assignment DPM analysis with fixed baseline of weakfish juveniles. The middle circle indicates a co-assignment probability of $p=0.05$. Five main clusters are evident and marked by the corresponding baseline sample origin (North to South): NY = New York (grey), DE = Delaware (blue), CB = Chesapeake Bay (green), NC = North Carolina (red) and GA = Georgia (yellow). A geographical gradient in chemistry is evidenced by predominant miss-classification to neighbouring estuaries.

Second, a set of tests was conducted for which I removed juveniles from each of the baseline sources in turn, subsequently using these juveniles as the mixed sample. I further repeated this test with all combinations of any two sources removed from the baseline. This test serves to see whether new sources are created when they should be (i.e. 


\section{Chapter 3. Bayesian mixture models for otolith geochemistry}

when extra baseline sources are present in the dataset) and if the corresponding individuals will associate with this extra-baseline source. Lastly, I used this model to assign adult weakfish to natal sources, leaving the possibility for extra-baseline sources. For these scenarios I used the same prior as before, this time however I placed a Gamma $(1,1)$ prior on $k_{0}$. Escobar \& West (1995) noted that most often there will not be enough information in the data to estimate this parameter - the inclusion of a baseline however should provide some information about the scale of the variance around the individual source means. Since information from the baseline is now available, the baseline prior for the co-variance matrix was now the mean within source covariance matrix.

The 'cross-validation' assignments of fish from a single source, with that source omitted from the baseline, shows that the method works very well for most sources (Table 3.2): the vast majority of fish are assigned to a single extra-baseline source. DE is (not surprisingly) again the exception with multiple predicted extra-baseline sources, two of which account for most of the individuals due to the above mentioned multi-modality of that source. The number of predicted sources is often slightly above the actual number, highlighting the need to actually look at the number of fish within each source to make statements about the number of contributing sources (see also White et al. 2008). Results with combinations of two sources omitted from the baseline show very similar patterns: most individuals are assigned to mostly two distinct extra-baseline clusters (average proportion $\bar{p}=0.96$, average modal number of predicted sources $\overline{\operatorname{mode}\left(K^{+}\right)}=6.69$ ). 
Table 3.2: Results from the assignment test, excluding each source from the baseline and using the DPM with fixed remaining baseline to assign the fish from the excluded source. The table shows the posterior expectation of the total number of sources after classification $\left(E\left(K^{+}\right)\right)$, the posterior mode of the number of extra-baseline sources $\left(\operatorname{mode}\left(K^{+}>K_{B}\right)\right)$, mode $\left(n\left(K^{+}>K_{B}\right) / N\right)$, the posterior mode of the proportion of assigned fish associated with an extrabaseline source and mode $\left(n_{\max }\left(K^{+}>K_{B}\right) / N\right)$, the number of fish assigned to the largest extra-baseline source.

\begin{tabular}{|c|c|c|c|c|c|}
\hline & $\mathrm{NY}$ & $\mathrm{DE}$ & $\mathrm{CB}$ & $\mathrm{NC}$ & $\mathrm{GA}$ \\
\hline$E\left(K^{+}\right)$ & 5.94 & 7.53 & 6.10 & 5.25 & 5.03 \\
\hline $\operatorname{mode}\left(K^{+}>K_{B}\right)$ & 2 & 4 & 2 & 1 & 1 \\
\hline $\operatorname{mode}\left(n\left(K^{+}>K_{B}\right) / N\right)$ & 0.98 & 0.94 & 1 & 0.96 & 0.87 \\
\hline mode $\left(n_{\max }\left(K^{+}>K_{B}\right) / N\right)$ & 0.90 & 0.56 & 0.98 & 0.96 & 0.87 \\
\hline
\end{tabular}

Finally, the DPM with baseline was applied to the weakfish data. I first applied the DPM with a fixed baseline (i.e. the juvenile data are not clustered, but fish from each source are co-assigned with probability one) since this most closely conforms to a traditional mixture analysis - with the added possibility for additional sources. The resulting estimate of the number of sources in the complete dataset is substantially higher than the number of baseline sources $\left(\operatorname{mode}\left(K^{+}\right)=11\right)$. I showed above that some outliers can at times lead to an over-estimation of sources. Here, however, all extra sources have a significant number of adults assigned to them (Figure 3.4). The exact linkage tree displays 11 distinct clusters with coassignment probabilities smaller than 0.05 , we thus have at least 6 distinguishable extra-baseline sources. Furthermore, very few adults actually group with baseline samples, and then only with relatively low probability. Within adults however there is strong evidence that many fish collected within some estuaries come from relatively few sources. Adults collected in NC for instance cluster into two main groups. Most fish from 
GA group as one large cluster, indicating that most adults found in this estuary originate from a single source. The same holds for NY adults. CB and DE adults however display only a slight amount of clustering and are found in most extra-baseline source clusters, though the co-assignment with fish in these clusters is usually low for fish from these estuaries.

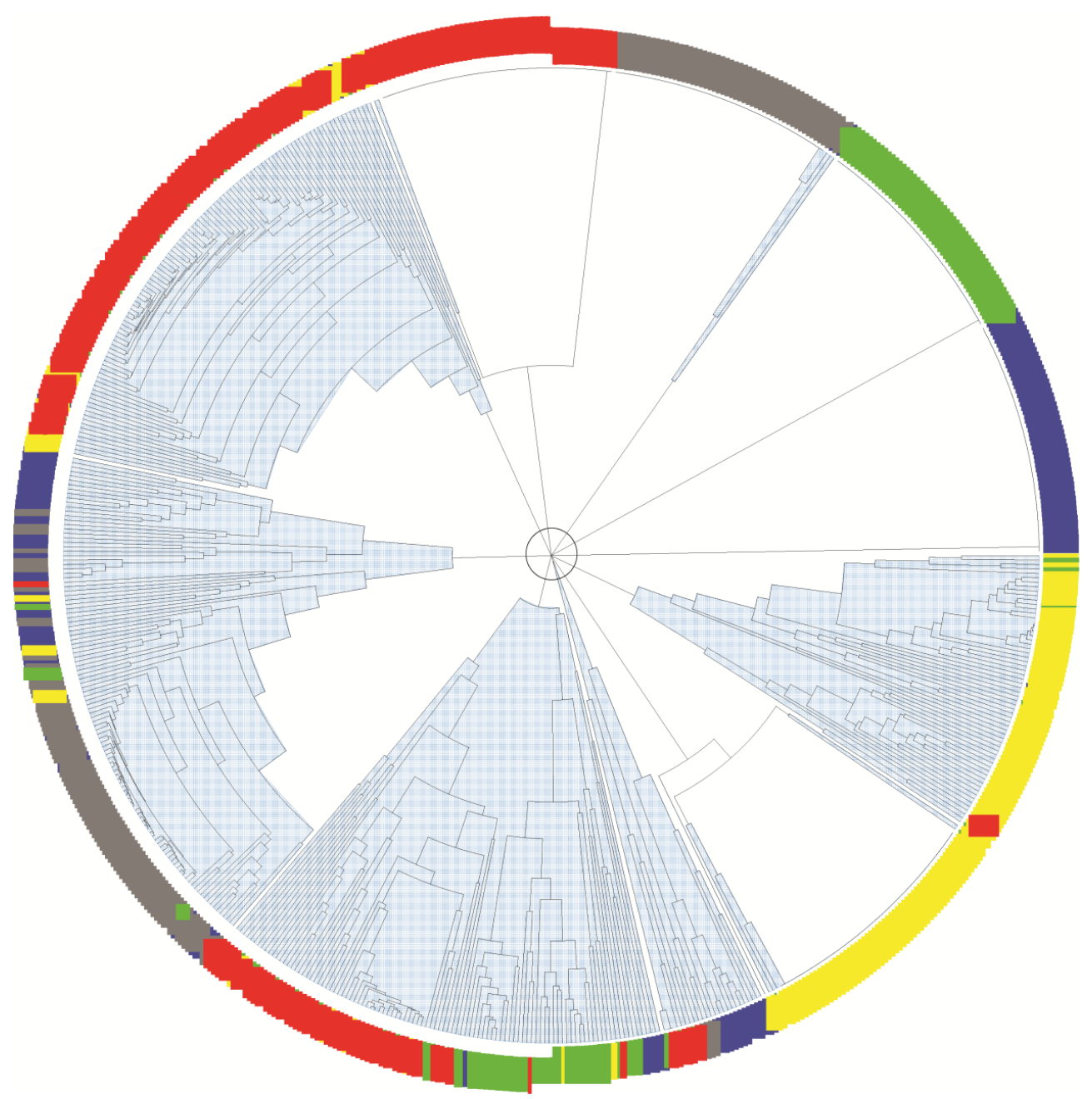

Figure 3.4: Weakfish exact linkage tree from the DPM clustering model with fixed baseline. Sub-trees including adult weakfish are highlighted in light blue. Leaf colours indicate collection location (North to South): New York (grey), Delaware (blue), Chesapeake Bay (green), North Carolina (red) and Georgia (yellow). 


\subsection{Application}

In the light of these results, I re-analysed the data, using the DPM for clustering juvenile baseline samples together with adult samples (i.e. the juvenile data is now not necessarily as separate source clusters). The picture is decidedly less separated (Appendix A1): while there are still a number of extra-baseline sources $\left(\operatorname{mode}\left(K^{+}\right)=10\right)$, many fish also cluster more closely with baseline samples from their estuary of collection, supporting the homing hypothesis. This is particularly evident for NC fish, although a large proportion of NC adults cluster on their own. Although not particularly evident from the tree (due to overlap of labels to keep the tree at a manageable size), some Chesapeake Bay samples also cluster within baseline cluster predominated by NC juvenile signatures. Others from this estuary cluster on their own with baseline fish from the same collection location. Some Georgia adults are found with the corresponding baseline signatures, while others cluster on their own. Fish also cluster with fish from neighbouring estuaries on many occasions, but as shown above, this may as well be due to miss-classification. Most strikingly, fish from DE and NY often group together, both for adults and juveniles, but form separate clusters for these age classes.

\subsubsection{Classification with a (potentially) incomplete baseline}

The DPM clustering results for the weakfish data (section 3.3.3) demonstrate that there are likely extra-baseline sources present in the adult dataset, which are distinct from those sampled for the baseline. Thorrold et al. (1998) report that even within estuaries there are sufficient differences in otolith signatures. Given that only a maximum of three locations were sampled within each estuary, and given the catch statistics for weakfish, one could assume that these extra-baseline sources are most probably from other sites within any one or some of these estuaries. I therefore applied the classification method for incomplete baselines to this dataset, classifying adult fish to the, potentially incomplete, juvenile baseline. Rather than keeping the baseline fixed, I clustered the baseline 
with adult samples within each estuary since i) the pooled baseline within each estuary seems to poorly represent the sampled locations (c.f. results from the DPM with fixed baseline, section 3.3.3) and ii) this seems to reveal more fine scale patterns.

First however, I validated the classification method with a set of tests. To this end I added my own dataset (Neubauer et al. 2010) as a more difficult and spatially hierarchical test set. In this dataset, I found geographical variation of chemical signatures from hatchlings of common triplefin (Forsterygion lapillum, Tripterygiidae) mainly between two main islands and sites spread across these islands. A detailed study using this classification method to assess connectivity between triplefin subpopulations will be presented in Chapter 4, and I limit myself here to the validation and illustration of the method's features.

To assess whether the model performed its classification task in an ideal situation in which the baseline is completely characterized, I performed classification using self-assignment. Classification performance was compared against the commonly used linear discriminant function classification. To make sure that results were not dataset dependent, I performed the same test on the weakfish baseline. I used fixed baselines for the triplefin dataset because it allowed me to compare classification success at the site specific scale against my earlier results. For the weakfish data I clustered the baseline with the mixed sample within each region because this seems to give more sensible results in the clustering application and helps to demonstrate that the method works both ways. 
Table 3.3: Comparison of the proportion $\mathrm{P}_{\mathrm{c}}$ of correctly assigned individuals in a self-assignment test for the weakfish and triplefin datasets. The DP classification scheme is abbreviated as DPMc, LDA stands for linear discriminant analysis. K indicates the number of baseline groups at each level of the classification.

\begin{tabular}{|c|c|c|c|c|}
\hline & \multicolumn{2}{|c|}{ Classifier } & $\mathrm{K}$ & $\mathrm{P}_{\mathrm{c}}$ \\
\hline \hline \multirow{3}{*}{ Weakfish } & \multicolumn{2}{|c|}{ DPMc } & \multirow{2}{*}{5} & $\mathbf{0 . 9 7}$ \\
\cline { 2 - 3 } \multirow{3}{*}{ Triplefins } & \multicolumn{2}{|c|}{ LDA } & & 0.86 \\
\hline \hline \multirow{3}{*}{ DPMc } & Island & 2 & $\mathbf{0 . 8 7}$ \\
\cline { 3 - 5 } & & Site & $8 / 9$ & $\mathbf{0 . 5 2}$ \\
\cline { 2 - 5 } & \multirow{2}{*}{ LDA } & Island & 2 & 0.81 \\
\cline { 3 - 5 } & & Site & $8 / 9$ & 0.34 \\
\hline
\end{tabular}

Table 3.3 shows that the classification procedure works very well for both datasets in an idealized situation; i.e. when the baseline if fully characterized. Since the motivation for this method is the possibility of an incomplete baseline at any level, a more relevant test scenario is one where some of the baseline is omitted. I conducted two sets of such tests for the triplefin dataset, leaving out fish from two sites and five sites for each set, respectively. Sites were chosen at random and the test was repeated 5 times for each test, using the full baseline as the mixed sample. The proportion of correctly assigned individuals is now a sum over the proportion of individuals corresponding to the incomplete baseline being correctly classified to the remaining sites within each region, and the proportion of fish corresponding to the omitted sites in the baseline which were assigned to extra-baseline sources. The results from this test confirmed that the classification success drops when sites are omitted, but stays within reasonable bounds. Omitting two sites lead to an average assignment accuracy of 0.80 . Omitting five sites corresponded at times to a reduction of the baseline to only about half its size, and it lowered the accuracy to about 0.74 . Lastly, I assessed the bias in the classification method due to extreme proportions in the mixture. Such situations commonly lead to bias in estimates in mixing proportions. Using 5 hold-out sets of 25 and 5 individuals from both Island sources respectively, I 
assessed whether the estimated proportions correspond to those of the hold-out set. The average proportion over the 5 simulations were 0.78 and 0.22 respectively, indicating that even for very uneven proportions the method's estimates are relatively close to the true value.

Finally, classification of weakfish data lends strong support to the homing hypothesis; I find that all collected adult fish likely homed to their natal estuary (Figure 3.5). Within each estuary however, a number of distinct sources contribute to mixed samples from all estuaries. In most cases, the adults grouped with comparatively few fish from the baseline, which likely explains the extra-baseline allocation of those individuals in the DPM clustering with a fixed baseline. Co-assignment probabilities (CP) of adults with their baseline were generally highest for NY samples (Figure 3.5a), although a small subset (3) of these fish groups with only few baseline samples and at low CP and could be misassigned. The same holds for a group of adults in DE for whom CPs with other DE fish are low (Figure 3.5b) Co-assignment probabilities for all other locations are similar to the variability found within the baseline, indicating that the clusters are robust. Lower CPs are generally a sign of more uncertainty about the actual number of sources within regions: $\mathrm{CB}$ for instance is divided into a number of distinct sources with low CP. This is perhaps not surprising given the size of $\mathrm{CB}$ relative to the scale at which otolith signatures vary (i.e. between individual collection often sites (Thorrold et al. 1998)). 


\subsection{Discussion}
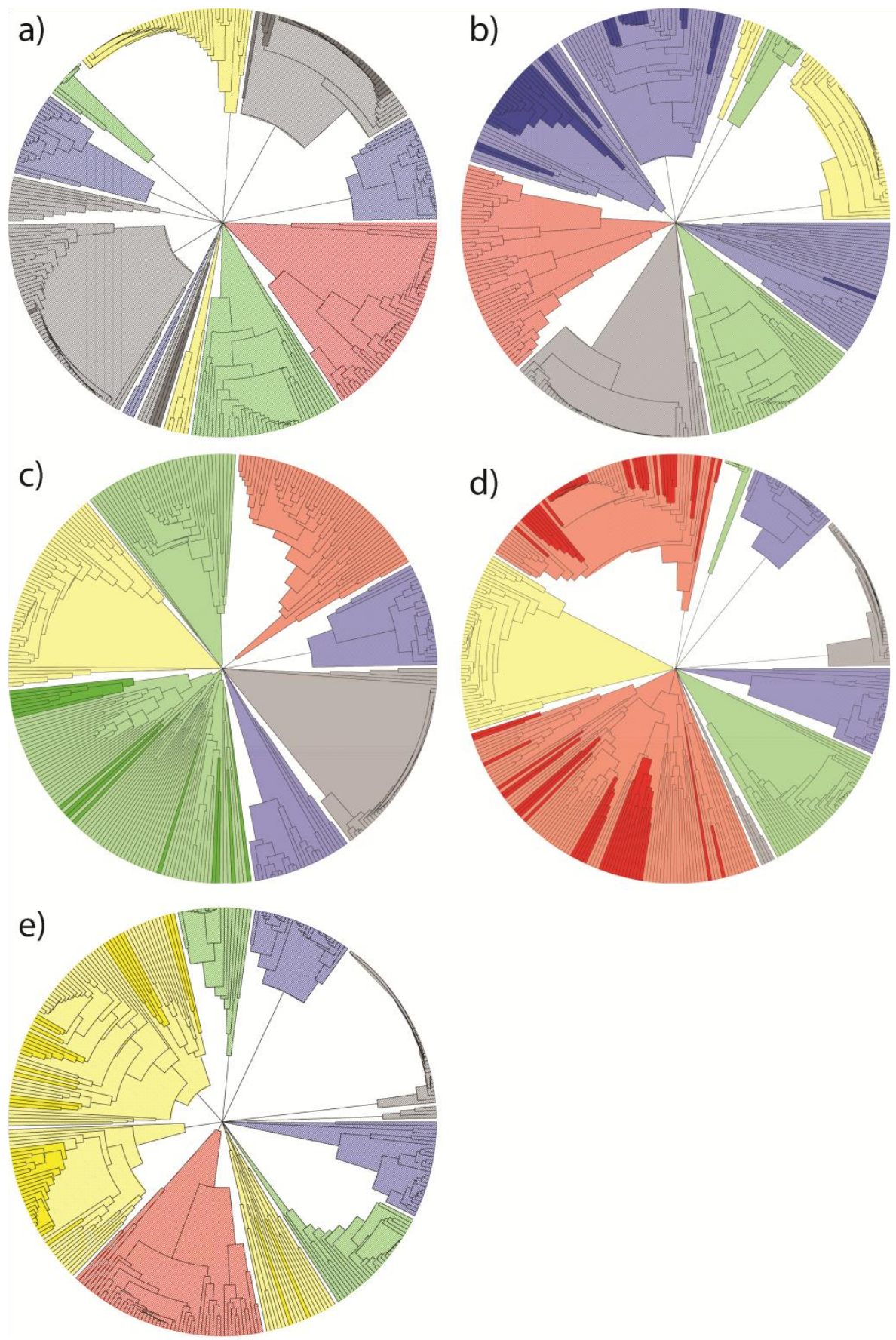

Figure 3.5: Exact linkage trees from the DPMc classification procedure. Adult fish are coloured in darker shades whereas the juvenile baseline is represented by lighter shades. Note that the shading only represents a subset of the clustered adults: only if two or more adults group together are these discernible in the tree. The trees are therefore for illustration purposes only. Tree colours indicate collection location for both adult and juveniles whereas individual panels are adult collection estuaries (North to South): a) New York (grey), b) Delaware (blue), c) Chesapeake Bay (green), d) North Carolina (red) and e) Georgia (yellow). 


\section{Chapter 3. Bayesian mixture models for otolith geochemistry}

\subsection{Discussion}

In this chapter I developed a modelling approach which simultaneously addresses a number of problems with the use of geochemical signatures to estimate dispersal and migration. I showed how the number of sources can be efficiently estimated using DP methods when a baseline is present. Both clustering and classification procedures based on these methods provide considerable insight into patterns in geochemical data obtained from otoliths or comparable sources of geochemistry. The DPM as stated above essentially performs a clustering of the data while building a distribution over the exact number of modes (=sources) in the data. This may in fact be a difficult task with otolith chemistry when no baseline is present: one will have to be sure that the definition (i.e. the geographical scale) of a source corresponds to a unimodal (normal) distribution. The biggest limitation to a use of the DPM without a baseline in that respect is thus the interpretability of results on a geographical scale.

This new modelling approach has several advantages over existing methods for discovering structure in a recruit pool based on otolith chemistry. Current methods that use otolith chemistry to uncover the number of sources in a recruit dataset or a mixed fishery use model selection or re-sampling criteria to produce a single best model (White et al. 2008, Fontes et al. 2009). The DPM model produces a marginal distribution over the number of sources, the direct probabilistic interpretation of which is more natural than that of arbitrarily scaled model selection criteria such as the AIC or DIC. Furthermore, the marginal descriptions of relatedness of individual fish, expressed by the coassignment probabilities, have a natural interpretation and allow for a more thorough exploration of the structure of the mixture and the baseline. Finally, this method can be readily extended to include genetic characters by combining it with the model proposed by Pella \& Masuda 


\subsection{Discussion}

(2006) (i.e. the likelihood for a source becomes the product of genetic and otolith likelihoods (Smith \& Campana 2010)). It can also be extended to many-to-many analysis as detailed in Bolker et al. (2007).

The new classification procedure presented in this paper can be thought of as a more flexible way to model the baseline which underlies the classification rule. The assumption of a single normal distribution for each source or region may in fact be misguided in many applications and lead to overlapping distributions and hence lower classification accuracy. This is also the likely reason why the non-parametric random forest classification method performed better than parametric LDA in a recent comparative analysis of classification methods for otolith data (Mercier et al. in press). None of these methods go beyond simple classification however, and they suffer from the same problem as LDA, notably that the source proportions are assumed to be known and constitute a prior in the analysis. This can lead to poor classification performance in datasets with extreme source proportions in the mixed sample (White \& Ruttenberg 2006).

The classification model presented here is akin to mixture discriminant analysis (Hastie \& Tibshirani 1996), where each class is taken to arise from a mixture of a known number of normal distributions. The difference is that the number of mixture components (sources) in the DPM model is inferred directly from the atlas data, and unknown test samples may come from unspecified components. Seen in this light, the DPM modelling approach seems ideally suited to accommodate complex multimodal distributions that can arise in otolith geochemistry through the interplay of maternal effects and environments influences, which may have different scales for different elements. This is reflected in considerably higher classification accuracy at the lower level (i.e. sites in the triplefin dataset). The clustering procedure at the lower level of this framework allows us to 
cluster without a priori division into sites, which provides a method to assess whether the clustering is clear cut or poorly resolved at smaller spatial scales. The possibility for recruits to come from un-sampled sites make the DPM and DPMc approach an excellent tool for exploring connectivity based on geochemical tracers in geographically and chemically complex landscapes. It is thus a more realistic procedure in most otolith applications in the marine environment where the assumption of a complete baseline can rarely be justified.

Classification of the weakfish data confirmed the suspicion from the self-assignment test with the DPM cluster model: I found that classification to neighbouring estuaries reported in earlier studies was likely due to misclassification and that natal homing is likely near perfect. The genetic homogeneity of the stock indicates that some dispersal between estuaries must happen, but since very few migrants per generation are enough to maintain a genetically homogenous population, this number could be next to undetectable with manageable sample sizes for otolith geochemistry.

One alternative explanation for the perfect homing pattern observed here is that many adult weakfish signatures are sufficiently different from the bulk of the baseline signatures. Given that allocation to any of the baseline estuaries would then be equally unlikely, the classification would be driven by the allocation of a few individuals to a baseline sample, which would influence the estimates of mixing proportions which in turn dominate the likelihood for any estuary. In simpler terms: if the model is very uncertain about where some adults may have come from, then these fish are more likely to be assigned to the region to which most other fish have already been assigned. This would seem like a likely explanation in the light of results from the DPM clustering model with fixed baseline: most adults cluster together and apart from the baseline. Allowing the baseline to be clustered with adults however shows that this may be an 


\subsection{Discussion}

artefact of pooling sites within estuaries, and that many adults cluster as closely with the baseline as they do amongst themselves. For DE and NY many adults cluster together and apart from juveniles, but this is not a generality. The classification trees confirm this: adults usually cluster with the baseline in a number of distinct clusters and with comparable CP to those seen between baseline samples. The exception may be a relatively large group of adults in DE and a small group from NY for which CP with their baseline were relatively low.

All models developed in this chapter could be applied to other types of geochemical tracers, such as stable isotopes, as well as related techniques using other calcified structures or even soft tissue trace elements. The approach presented here is a general Bayesian (fuzzy) clustering and classification framework which may even be applied to unrelated problems for which clustering based on probabilistic assumptions seems advantageous. In this respect the model and hierarchical tree building method employed here is related to Bayesian hierarchical clustering (Heller \& Ghahramani 2005), which aims to directly construct a hierarchical clustering tree from data based on the same probabilistic assumptions. The node height in Bayesian hierarchical clustering is however not directly interpretable in probabilistic terms - a feature which seems a clear advantage of the present framework. In conclusion, this chapter develops flexible and useful methods to be used with otolith chemical signatures. It is shown to perform well - especially with regard to estimating missing sources in the baseline dataset, which up until now had been a significant limit for the use of otolith geochemistry in complex environments. The DPM methods therefore provide a considerable step towards a more general applicability of geochemistry for questions of dispersal and migration. 


\section{CHAPTER 4}

\section{Larval dispersal pathways in a reef fish metapopulation: Contrasting empirical and}

simulation measures

\subsection{Introduction}

Dispersal of propagules and adults in fragmented animal populations (metapopulations) is a major determinant of population dynamics (Hanski 1999, Kendall et al. 2000, Gaines et al. 2007, Gouhier et al. 2010). The spatial configuration of populations and their ensuing connectivity will further determine the persistence of local populations and the viability of the metapopulation network as a whole (Hastings \& Botsford 2006, ArtzyRandrup \& Stone 2010). Connectivity measures are therefore vital for resource management (Fogarty \& Botsford 2007) as well as conservation planning (Crooks \& Sanjayan 2006).

In the marine environment, dispersal of larvae between local populations can link dynamics of marine populations and communities over large geographical scales (Gaines et al. 2007, Gouhier et al. 2010). While the importance of connections has been much discussed in theory (Armsworth 2002, Hastings \& Botsford 2006, Gouhier et al. 2010) and in the light of conservation questions (Botsford et al. 2001, Guichard et al. 2004, Baskett et al. 2007), empirical estimates of connectivity in marine 


\subsection{Introduction}

metapopulations have been difficult to obtain (Cowen et al. 2007). As a result, limited information about dispersal patterns has contributed to uncertainties in spatial management of marine resources and the optimal implementation of marine reserves and reserve networks (Sale et al. 2005, Halpern et al. 2006). Unless we obtain reliable estimates of demographic connectivity in marine communities, we cannot predict whether reserves or spatial management actions will serve their desired purpose (Stobutzki 2001).

Many studies emphasize the importance of local hydrodynamics in deciding dispersal outcomes and their demographic consequences (Black \& Moran 1991, Guichard et al. 2001, Gilg \& Hilbish 2003, Byers \& Pringle 2006, Swearer \& Shima 2010, White et al. 2010). In particular, variability and heterogeneity in flow fields are not only responsible for spatially heterogeneous connectivity (Mace \& Morgan 2006, Mitarai et al. 2008, Siegel et al. 2008, White et al. 2010), they also provide a means for population maintenance in directional (advective) flows (Largier 2003, Byers \& Pringle 2006). Given these results, variability in flow may actually be as important a determinant of population structure and connectivity as mean flow patterns.

Specifically, marine organisms are predicted to adapt to exploit such heterogeneities (Byers \& Pringle 2006) and many larvae indeed show species-specific patterns of utilizing spatially heterogeneous flow (Breitburg et al. 1995, Paris \& Cowen 2004, Morgan \& Fisher 2010). In the face of such potential adaptations, it will be difficult to make specific predictions about dispersal patterns from hydrodynamic models alone. Such models are important for developing hypotheses about potential dispersal pathways, especially when paired with species-specific behavioural information (Paris \& Cowen 2004, Koehl et al. 2007, Leis 2007). 
Empirical data is needed however to confirm if such simulated dispersal pathways are realized within the metapopulation.

Recent advances in tagging methods such as trans-generational marking of fish otoliths (ear stones) and genetic paternity testing have led to considerable empirical insights into larval dispersal patterns, such as those reported for a marine reserve network in Papua New Guinea (Almany et al. 2007, Planes et al. 2009). However, since these methods necessitate the recovery of marked individuals, such methods are limited if population numbers are high and dispersal may occur over considerable scales. Natural population markers such as allele frequencies and otolith markers seem more applicable in such situations, but have been hampered by the necessity to sample all potential sources that may contribute to a sampled site. Often, such extensive sampling is not feasible in the marine environment. New statistical advances (Pella \& Masuda 2006, Chapter 3) now permit the analysis of genetic or geochemical patterns under the assumption of incomplete baselines, making methods involving natural population markers more applicable and realistic in such situations. With these novel approaches, we can potentially distinguish extra-baseline sources from sampled sources within the dataset (provided the two have different signatures).

In this chapter I investigate potential dispersal pathways of common triplefin (Forsterygion lapillum) fish larvae amongst a set of marine reserves in Cook Strait, New Zealand using otolith geochemistry. Since the baseline atlas (see chapter 2 ) is most likely incomplete, I apply the aforementioned statistical methods, for the first time, to geochemical otolith core signatures of fish that recently recruited to these reserves. Furthermore, I use an adaptive grid hydrodynamic model (Popinet 2003, Popinet \& Rickard 2007) which simulates passively dispersing larvae to make qualitative predictions about potential dispersal pathways in Cook 


\subsection{Methods}

Strait, New Zealand. The grid refinement of the model is adaptive and dynamic, which makes it particularly suitable for modelling in areas of complex flow where fixed grid approximations may be impractical due to implement due to very high computational requirements at a reasonable resolution. To gain insight into realized dispersal pathways and potential mechanisms of connectivity in this system, predictions from the simulation model are then compared with empirically inferred dispersal events.

\subsection{Methods}

\subsubsection{Study system and otolith core chemistry atlas}

I established a reference atlas of hatchling (core) otolith signatures from 17 sites around Cook Strait, New Zealand (Figure 4.1) by sampling otoliths of newly hatched larvae of the common triplefin (Forsterygion lapillum), a reef fish that is commonly found in sheltered shallow reefs around New Zealand (Clements 2003, Feary \& Clements 2006). Its relatively long larval duration of $\sim 52$ days (Shima \& Swearer 2009a) makes it a good candidate to study dispersal in marine metapopulations. Our collection sites for the reference atlas are distributed hierarchically across multiple regions on either side of Cook Strait, which separates the two main Islands of New Zealand (Figure 4.1). Further details about analytical methods, the atlas and the study species can be found in Chapter 2 .

\subsubsection{Recruit otolith preparation and pre-processing}

Between 50 and 62 days after collecting hatchlings from the aforementioned sites, I collected a total of 169 recruits of $F$. lapillum in three marine reserves around Cook Strait, New Zealand (Figure 4.1), namely at Taputearanga Marine Reserve in Wellington (60 recruits), Long Island Marine Reserve, Queen Charlotte Sound (59 recruits) and Kapiti Island, upstream of Cook Strait (40 recruits). 


\section{Chapter 4: Larval dispersal pathways in a reef fish metapopulation}

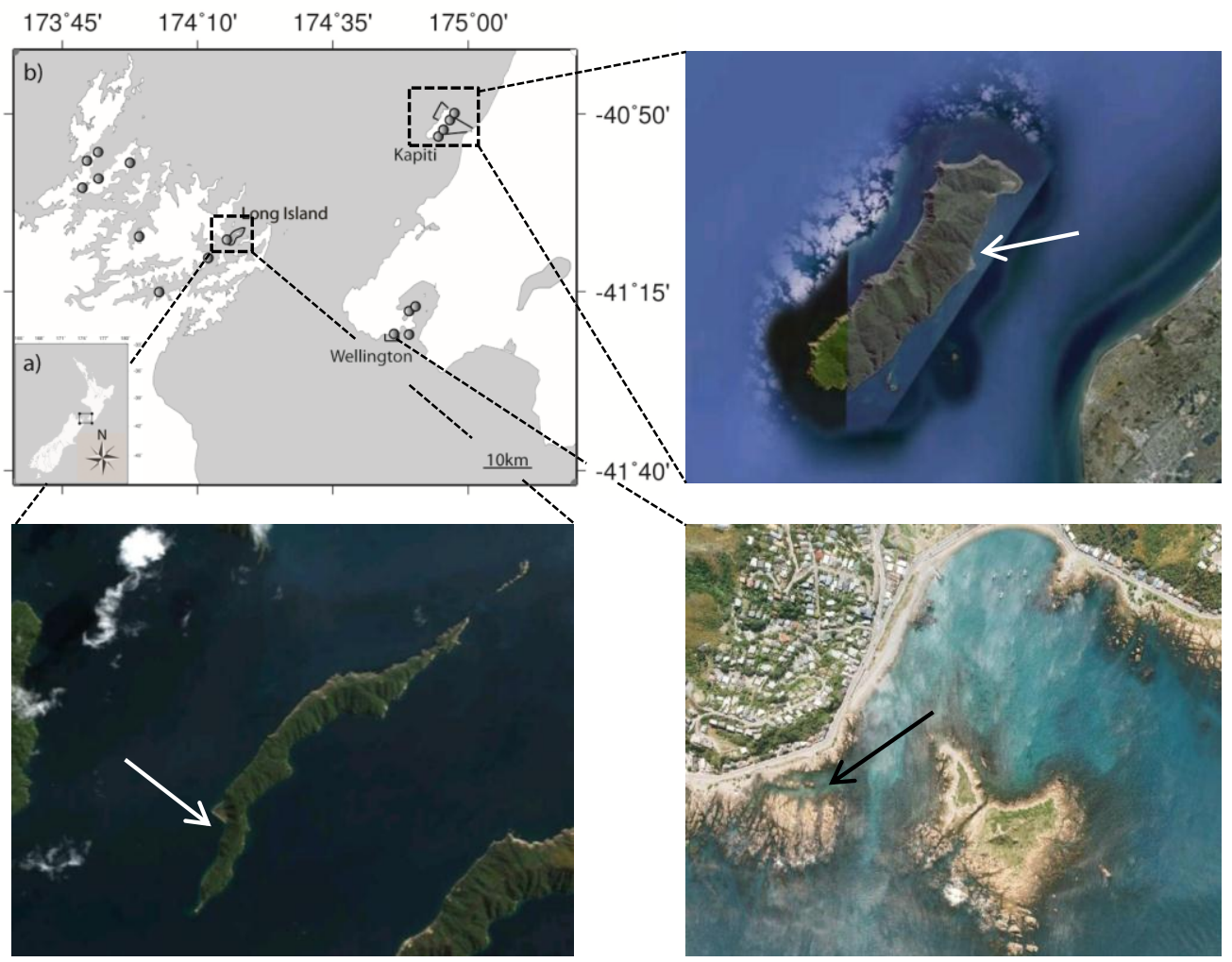

Figure 4.1 : a) Map of Cook Strait, New Zealand and b) the sampling domain. Our 17 baseline sampling sites (grey circles) are scattered either side of Cook Strait. Black outlines mark borders of regional marine reserves where recruits were collected (arrows on blow-up aerial photographs).

Taputearanga marine reserve in Wellington is located on the southern coast of the city of Wellington, at proximity to the opening of Wellington Harbour (Figure 4.1). Wellington's south coast is characterized by its exposure to southerly swells which batter the coast throughout the year. Local populations of breeding F. lapillum are found in this reserve only in a few sites which are sheltered from swell by Taputeranga Island, a small island about $50 \mathrm{~m}$ offshore in Island Bay, Wellington.

Kapiti Island and its associated marine reserve are located $\sim 50 \mathrm{~km}$ north of Wellington and approximately $5.6 \mathrm{~km}$ offshore (Figure 4.1 ). Bathed in waters of the D'Urville current which pushes water south-eastward through Cook Strait (Heath 1986, Chiswell \& Stevens). F. lapillum breeds in 


\subsection{Methods}

the relatively sheltered eastern waters of the Island, at isolated sites that are free from otherwise ubiquitous brown algae.

Long Island reserve is located in the opening of Queen Charlotte Sound in the Marlborough Sounds on the upper South Island of New Zealand (Figure 4.1). Little swell reaches this island, however, strong tidal currents and strong northerly wind-chop restrict breeding sites for my study species to very few small patches close to the shores of the island.

Immediately after collection these recruits were placed in vials containing $95 \%$ analytical grade ethanol. All otolith handling material was rinsed in weak acid for $24 \mathrm{~h}$ before being used in the otolith preparation procedure. Saggital otoliths were extracted using plastic tweezers and were subsequently placed into well plates for handling. Extractions and handling took place in an ultra-clean positive pressure laboratory and under a class 100 laminar flow hood. After extraction, the otoliths were subjected to two consecutive rinsing baths in $15 \% \mathrm{NaOH}$ buffered hydrogen peroxide solution for $24 \mathrm{~h}$ each. Between and after each cleaning bath the otoliths were cleaned three times with pure $(18.2 \mathrm{M} \Omega)$ water. Air-dried otoliths were then mounted onto circular slides using chemically inert epoxy resin (Buehler Epothin; Buehler, Lake Bluff, Illinois, USA). Each otolith was polished to the core using $9 \mu \mathrm{m}$ diamond lapping film (3M) mounted on an 8 inch $(20 \mathrm{~cm}$ ) lapping wheel (Model 920; South Bay Technologies, San Clemente, California, USA) and again cleaned as described above following the polishing.

Chemical analysis of recruit otoliths followed methods described in (Shima \& Swearer 2009a). Briefly, I used Laser Ablation Inductively Coupled Mass Spectrometry (LA-ICPMS) to sample material corresponding to the core of recruits by ablating in a deepening pit using a $25 \mu \mathrm{m}$ laser spot. A spike in ${ }^{55} \mathrm{Mn}$ served as an indicator of core material (Brophy et al. 2004, Ruttenberg et al. 2005). A significant number of otoliths had to be 
discarded as these were either damaged, ground too far down below the core, or we failed to locate the otolith core in the recruit. A total of 69 samples remained for final analysis - 29 from Wellington's Marine reserve, 20 from Long Island Marine Reserve and 20 from Kapiti. Following the protocol for the hatchling atlas (see chapter 2), I averaged ten scans across this peak to obtain the natal signature.

\subsubsection{Selecting elements for analysis of otolith cores}

In order to ensure that the data I collected from the recruits is comparable to signatures from the reference atlas, I treated the mean and variance of log-transformed elemental distributions in the atlas samples as coming from a normal distribution of unknown characteristics. I then estimated these characteristics (mean and variance) using Bayesian inference. For background on Bayesian statistics and reasoning the reader is referred to Gelman (2003). Briefly, Bayesian statistics employ Bayes theorem for statistical inference. In particular, the Bayesian approach defines that the posterior distribution of unknown parameters given the data is proportional to the likelihood of the data times the prior distribution for the parameters (see also Chapter 1 ). The prior specifies a distribution which reflects our prior knowledge about model parameters. It can be shown that the posterior distribution is a weighted average of the information in the data at hand and the prior knowledge, where the weight of each is determined by their relative precision. Vague (or diffuse) priors (with large variance and hence very little weight) can be used when we have little or no information about the range of parameters to be estimated.

To encode the absence of prior knowledge about the distribution of signatures from the reference atlas, I placed a vague Gamma $\left(10^{-6}, 10^{-6}\right)$ prior on the precision and a vague normal prior $\mathrm{N}(0,1000)$ on the mean of the elemental distribution of all measured elements. I retained elements 


\subsection{Methods}

for which $>95 \%$ of recruit observations fell within the $95 \%$ credible interval of the estimated posterior distribution for each element. This analysis was performed using the WinBUGS (Lunn et al. 2000) software for Bayesian computation.

\subsubsection{Statistical analysis of otolith chemistry}

While my baseline sampling of potential natal sources covers most areas with significant local triplefin populations in Cook Strait (Smith 2008), it almost certainly does not cover all spawning sites. I therefore use the Dirichlet mixture classification model (DPMc, developed in chapter 3, section 3.2.3) to assign fish to natal origins. Using cross-validation on the baseline samples, I reported reasonable assignment success when classifying individuals to one of the two major New Zealand islands in Cook Strait (North vs. South island) (see chapter 2). For the purpose of this chapter I was interested in a more resolved picture. Therefore, I repeated the cross-validation tests (described in chapter 3 ) at the scale of regions within Cook Strait, as defined in chapter 2. In that chapter I found that regions were not significantly different, however the weak separation may have been due to grouping sites of very different characteristics into arbitrary regions.

Given that regions can be reasonably discerned with the DPMc (see Results), I classified fish to regions within Cook Strait, keeping the baseline sites as fixed entities (i.e. they are clustered with co-assignment probability $p=1$ ) as for the test examples in chapter 3 . In fact, I found in preliminary studies that clustering the baseline at the site level for this dataset often produced a great number of clusters, probably due to the strong clutch effect which outweighs any geographical pattern. Furthermore, this large number of clusters leads to extremely slow convergence of the Gibbs sampler. Thus, keeping the sites as fixed entities ensures that results remain interpretable at this level. 
To characterize classification probabilities, I used both posterior probabilities for regions, as well as posterior entropy, measured as the information entropy of the expectations of posterior assignment probabilities $\phi$ over all $K$ possible regions. The entropy is defined as

$$
H\left[\phi_{i}\right]=-\sum_{k=1}^{K} \phi_{i, k} \log \phi_{i, k}
$$

where $\phi_{i}=\sum_{s=1}^{S} p\left(\zeta_{i, s}=k \mid \theta\right)$, the approximated expected value of $p\left(\zeta_{i}=k \mid \theta\right)$ from $\mathbf{S}$ draws by Markov Chain Monte Carlo from this distribution. The information entropy measures the uniformity of a distribution: if the predictive distribution predicts every source $k$ with the same probability, this entropy is maximized with value $H_{\max }=\log K$. Vice versa, if the prediction is without uncertainty, the entropy will be zero. Dividing by $H_{\max }$ produces a measure of evenness $E=H / H_{\max }$ between zero and one. The posterior entropy thus provides a single measure to quantify the uncertainty in the classifications produced by DPMc. Lastly, clustering within each site is visualized using trees generated from marginal co-assignment probabilities to source clusters (see chapter 3, section 3.2.4), where the branch length of the trees is a direct measure of the probability that two fish originate from the same water-mass.

\subsubsection{Simulation models of dispersal}

I conducted two distinct simulation experiments using the Gerris flow solver, an adaptive mesh simulation model that solves the Boussinesq incompressible hydrostatic equations of ocean dynamics by making a 'shallow water' approximation which neglects the vertical coupling terms (Popinet 2003, Popinet \& Rickard 2007). This depth integrated model allows features of interest, such as eddies developing along the complex coastline of Cook Strait, to be adaptively resolved to a user specified resolution. Since the refinement is adaptive with respect to such specified features only, this resolution can be set to high values without the exponential increase in computational requirements usually found in fixed 


\subsection{Methods}

grid models. Mesh refinement was chosen to refine to a maximum resolution of $117.19 \mathrm{~m}$. The coastline was modelled with the same resolution, which was kept fixed for the topography.

A version of the Gerris ocean model has recently been applied to oceanographic modelling of tidal cycles in Cook Strait (Msadek 2005) and the present simulations are extensions of this work. The model domain includes Cook Strait as well as the Taranaki Bight and Tasman Bay to the west and Palliser Bay to the east. Msadek's (2005) work only included tidal forcing and neglected barotropic flow or any forcing due to wind. The present simulation contains forcing by wind fields, corresponding to the time of our empirical investigations. These were obtained from the NZLAM model (National Institute of Water and Atmospheric Research, New Zealand) at a resolution of $15 \mathrm{~km}$. A hyperbolic-tangent shaped gradient in sea surface height (SSH) was imposed to approximate observed differences between the Tasman Sea and the Pacific Ocean. The boundaries of the model domain were forced by SSH and current velocity values corresponding to the larval period of collected recruits, obtained from a wind driven NZ wide Regional Ocean Model (ROMS). The model was initiated on Jan $1^{\text {st }} 2008$ and ran for 52days. A separate manuscript describing model validation against drifters and acoustic Doppler current profiler data as well as a comparison to outputs from a ROMS model of the same region is in preparation and will be presented elsewhere (Neubauer et al. in prep).

I released passive tracers at locations and in densities which approximate those predicted by a model-generated distribution map of $F$. lapillum (Smith 2008). Release locations are between 3-10m in depth and confined to predefined squares within each region of interest (i.e. locations with significant abundance of F. lapillum within Wellington, Marlborough Sounds and Kapiti regions). Although F. lapillum can be found throughout 
NZ, it appears to be most abundant in sheltered regions of the Cook Strait, especially around Wellington and within the Marlborough Sounds. The model predicts little to no significant abundance of $F$. lapillum upstream of Cook Strait (i.e. north of Kapiti and west of the Marlborough Sounds as well as on the exposed eastern and western side of the northern South Island). This information was consistent with my own personal observations of common triplefin distribution in the Cook Strait region. I therefore assume that little, if any, dispersal into Cook Strait happens on an ecologically relevant timescale. Virtual larvae were released over a two week window starting on day 3 of the simulation. The simulation model outputs are employed to describe the movements of tracers in a qualitative way, in order to make hypotheses about potential dispersal pathways and mechanisms. These are then compared to dispersal routes predicted by otolith chemistry. A more detailed description of the Gerris ocean model including a detailed application of this model to simulations of larval dispersal will be given elsewhere.

\subsection{Results}

\subsubsection{Results from otolith microchemistry}

Detection limits and consistency standards for all measured elements are given in table 1. Most elements were above the detection limit for the majority of samples, however, $\mathrm{S}, \mathrm{Mn}, \mathrm{Cu}, \mathrm{Zn}$ and $\mathrm{Pb}$ were below detection limits for some fish. Comparison between the predictive distribution of baseline samples and sampled recruit cores revealed that $\mathrm{Mg}$ and $\mathrm{Mn}$ were significantly higher in hatchling cores than in recruit cores (Figure 4.2). These two elements were thus omitted from subsequent analyses (see the 'Discussion' section on maternal effects).

The hierarchical organization in the DPMc classification model developed in Chapter 3 improves classification success on the regional 


\subsection{Results}

scale to almost twice that of the linear discriminant analysis (LDA) used in chapter 2. Five cross-validation studies with 40 fish found an average accuracy of $67.6 \%$ from this method as compared to $34.9 \%$ mean accuracy with LDA.

I found distinct dispersal signatures in both the North and South Island recruits. Overall, associations at the site level as revealed by the exact linkage algorithm were rather low in terms of co-assignment probabilities with existing sites, indicating that either assignment to these sites is relatively uncertain and/or extra-baseline sites may often be present. Assignments at the regional level had mostly high posterior probabilities, although some ambiguous cases can also be found (Figure 4.3).

North Island recruits probably originated at both Wellington and Kapiti Island for both of these sites, indicating that connections exist in both directions (Figure 4.3a-d). Expectations of posterior assignment probabilities were usually lower for Kapiti Island than those for Wellington, indicating that there is some degree of overlap with other regions. Slightly more recruits to Kapiti Island marine reserve seemed to have come from Wellington and visa-versa, indicating that dispersal on that scale is rather common.

Recruits collected from Kapiti are grouped into two distinct clusters for each source region at the site level, indicating that multiple sites contribute to the recruit pool in the Kapiti marine reserve (Figure 4.3a-b). All clusters are associated with a baseline site: the larger cluster from Wellington is most closely associated with Wellington Harbour baseline signatures, while the smaller cluster is closest in similarity to Wellington coastal signatures. Most Kapiti sourced recruits associate with Northern Kapiti sites, from both within and outside the Kapiti marine reserve. 
Table 4.1: Distribution of trace element concentrations (in mol per mol Ca; note multiplier for each element column) relative to detection limits. Given are elemental concentrations for the quantiles that bracket $95 \%$ of all observations made from sampled otoliths, and the median value. Detection limits (DL in mol per mol $\mathrm{Ca}$ ) and external precision estimates (consistency standard, CS) for analyses of hatchling otoliths by LA-ICP-MS. DL estimates based on 20 blank analyses. Estimates of external precision are given in relative standard deviations (\%RSD) and are based on 11 blocks of samples (a block is a series of samples bracketed by standards), treating the NIST (National Institute of Standards and Technology) standard that is just below the standard used for calibration for each element as unknown and calculating the Element:Ca ratio for this standard for each block of samples.

\begin{tabular}{cc|ccc|ccc}
\multicolumn{2}{c|}{ Element } & \multicolumn{2}{|c|}{ Sample quantiles } & DL & CS & NIST(CS) \\
& & 0.025 & 0.5 & 0.975 & & & \\
\hline${ }^{7} \mathrm{Li}$ & $\left(* 10^{-6}\right)$ & 5.56 & 11.01 & 23.48 & 21.68 & 8.54 & 614 \\
${ }^{11} \mathrm{~B}$ & $\left(* 10^{-3}\right)$ & 0.092 & 0.16 & 0.20 & 0.062 & 9.14 & 614 \\
${ }^{24} \mathrm{Mg}$ & $\left(* 10^{-3}\right)$ & 0.11 & 0.25 & 0.53 & 0.021 & 7.83 & 612 \\
${ }^{31} \mathrm{P}$ & $\left(* 10^{-3}\right)$ & 0.60 & 1.17 & 4.01 & 0.14 & 13.27 & 612 \\
${ }^{34} \mathrm{~S}$ & $\left(* 10^{-3}\right)$ & 0.28 & 0.60 & 1.21 & 0.43 & 6.16 & 612 \\
${ }^{55} \mathrm{Mn}$ & $\left(* 10^{-6}\right)$ & 2.66 & 23.25 & 266.69 & 6.59 & 7.32 & 612 \\
${ }^{63} \mathrm{Cu}$ & $\left(* 10^{-6}\right)$ & 0 & 0.50 & 1.36 & 3.57 & 8.37 & 614 \\
${ }^{66} \mathrm{Zn}$ & $\left(* 10^{-6}\right)$ & 0 & 5.70 & 58.66 & 6.76 & 8.26 & 614 \\
${ }^{88} \mathrm{Sr}$ & $\left(* 10^{-3}\right)$ & 1.87 & 2.50 & 3.77 & 0.00093 & 7.48 & 612 \\
${ }^{138} \mathrm{Ba}$ & $\left(* 10^{-6}\right)$ & 1.92 & 13.15 & 56.08 & 0.088 & 7.77 & 614 \\
${ }^{208} \mathrm{~Pb}$ & $\left(* 10^{-6}\right)$ & 0 & 0.048 & 1.97 & 0.16 & 7.21 & 614 \\
\hline
\end{tabular}




\subsection{Results}

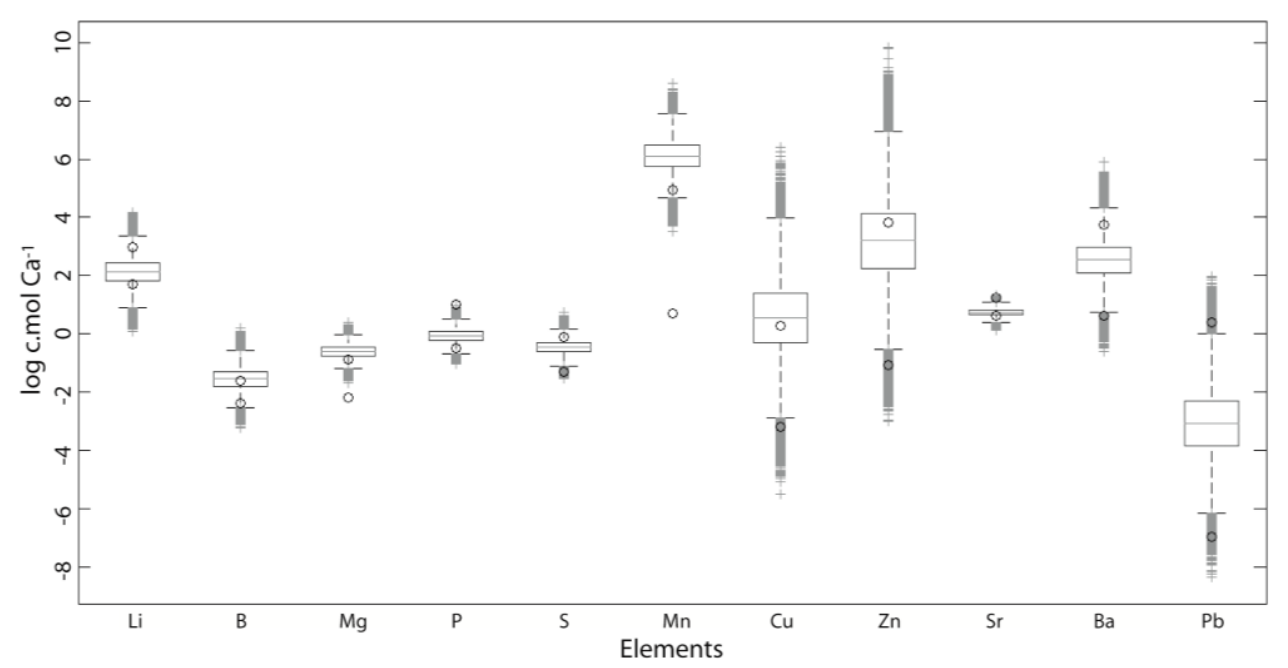

Figure 4.2: Hatchling atlas element distributions were estimated using a Bayesian approach (Boxplot); the wiskers indicate the $95 \%$ credible interval. the observed range of the recruit elemental signatures is delimited by open circles. Elemental concentrations are given in mol relative to calcium and are log transformed. Molar units for individual elements can be found in Table 1.

Recruits to Long Island marine reserve seem to be a mixture of fish spawned in Pelorus Sound and Queen Charlotte Sound (Figure 3e-f). Both clusters containing most recruits from Queen Charlotte Sound and Pelorus Sound are associated with sites from the hatchling baseline. Recruits originating from Queen Charlotte Sound most often associate with samples from within the sound, while those associated with Pelorus sound cluster with fish collected on the outer most site of Pelorus sound (Boulwer). 
a)

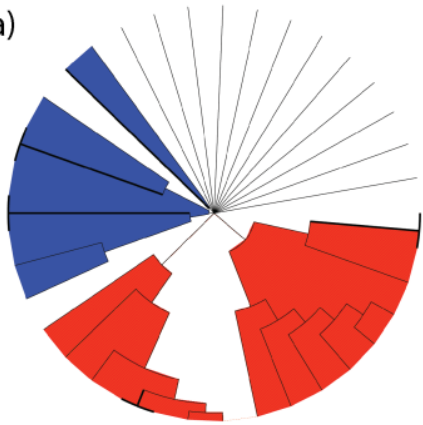

c)

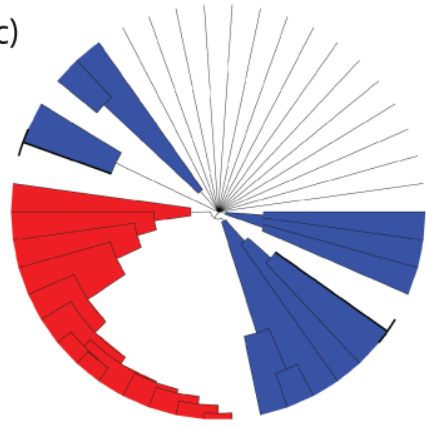

e)

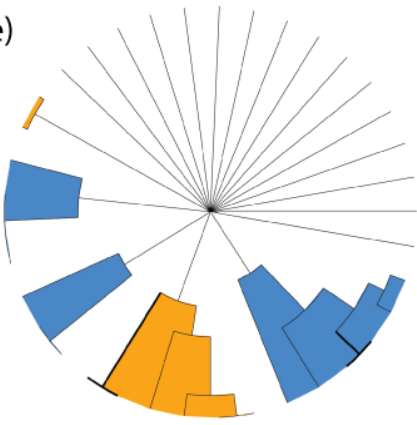

b)

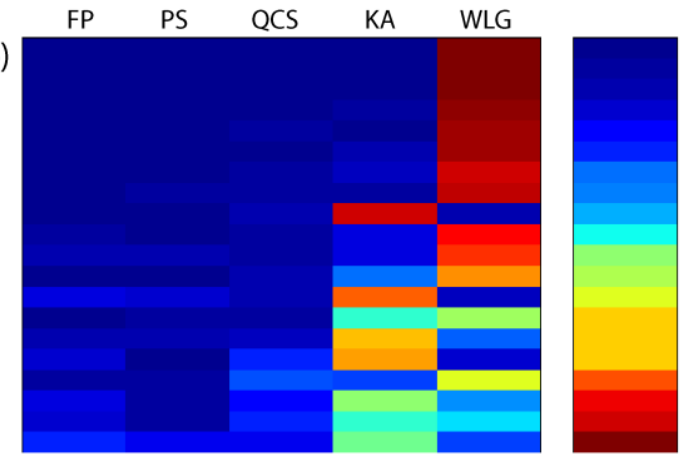

d)
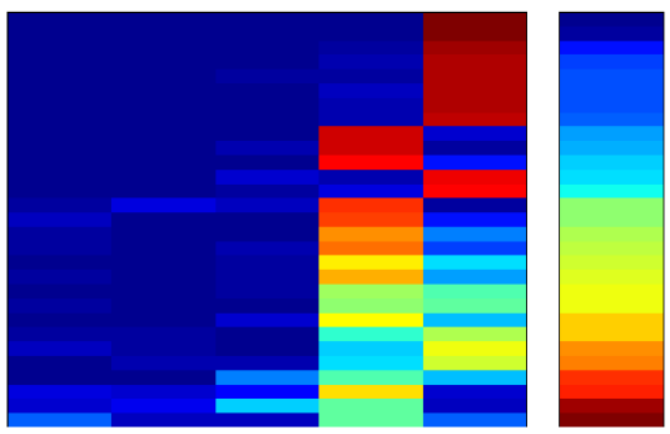

f)

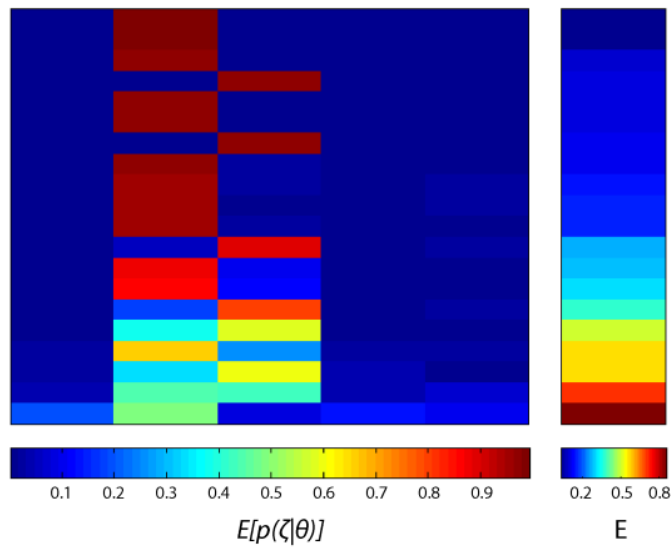

Figure 4.3: Assignment summaries for fish collected at the three reserve sites in Kapiti (a-b), Wellington (c,d) and Long Island (e-f). Co-assignment trees $(a, c, e)$ represent similarity in signatures: the node height in the tree is equal to coassignment probability to a source cluster (the sets of recruits/baseline signatures defined by a node). Branches that contain baseline site signatures appear in bold. Since the baseline was kept fixed at the site level (see Methods), these sites are collapsed to a single branch. Regional maximum posterior probability recruit assignments $(b, d, f)$ are coloured in the trees: French Pass (FP), Kapiti (dark blue KA in b,d,f), Wellington (red - WLG), Pelorus Sound (light blue - PS) and Queen Charlotte Sound (Orange - QCS). Expectations of assignment probabilities $(E[p(\zeta \mid \theta)])$ and corresponding evenness $E$ (see section 4.2.4) illustrate uncertainty in regional assignments $(b, d, f)$ for each fish (individual rows in each panel). Lower evenness translates as less ambiguity in assignments. 


\subsection{Results}

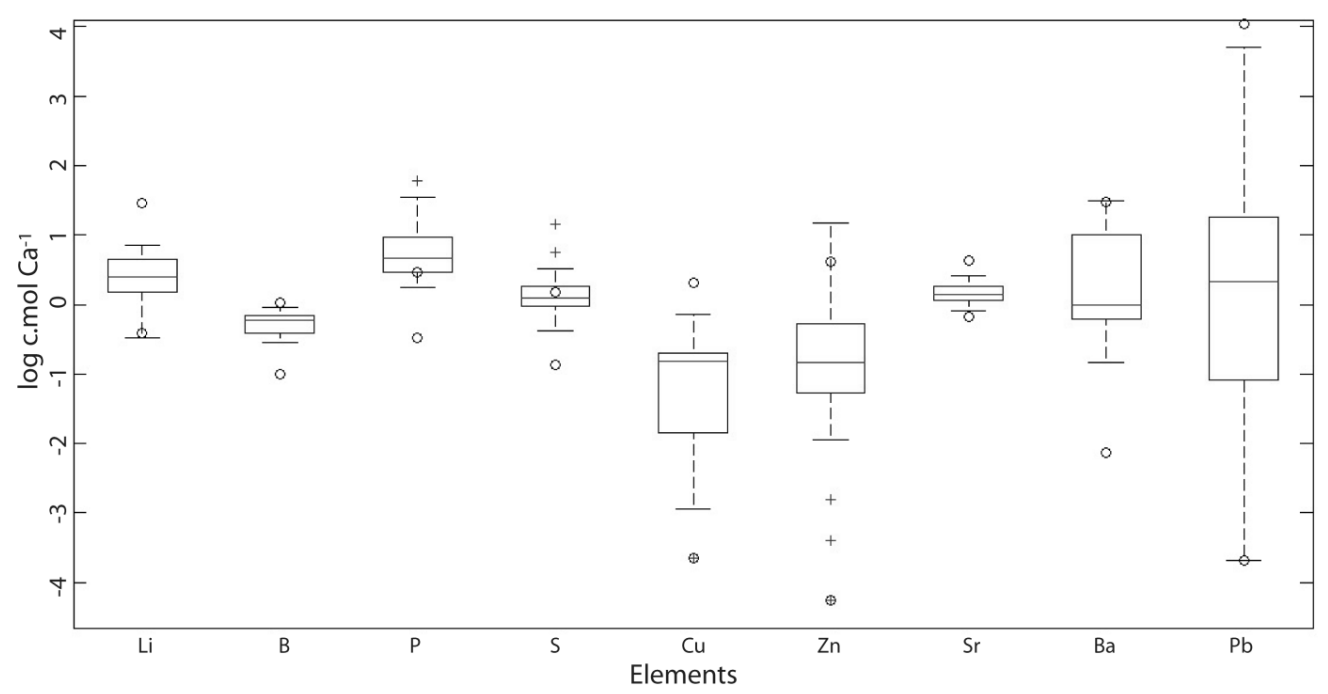

Figure 4.4: Comparison of log transformed signatures from samples in the isolated cluster of Wellington sourced recruits vs. all other recruits assigned to Wellington (in c.mol per mol of calcium, for multipliers see table 1). Remaining samples are represented by minimum and maximum values (open circles), samples from the cluster in question are represented by the box plot.

\subsubsection{Results from hydrodynamic simulation experiments}

The hydrodynamic model for Cook Strait predicts significant downstream dispersal as well as some nearshore upstream dispersal in Cook Strait (Figures 4.5-4.8). The dispersal pathways obtained from these simulations were similar across cohorts released over a two week window owing to strong mean currents and a long larval duration, and Figure 4.54.8 show results from a representative cohort. Tracers released in Kapiti move quickly downstream, though some seem to be naturally retained inshore (Figure 4.5). Tracers initially move towards the Kapiti coast (Figure $4.5 b)$, where a number of these tracers get retained near the coast for the extent of the simulations. Others move southwards (Figure 4.5c), along the north-eastern tip of the Marlborough Sounds and through Cook Strait towards Wellington and further south-eastwards. A substantial proportion of these tracers remain close to the entrance of Wellington Harbour for 


\section{Chapter 4: Larval dispersal pathways in a reef fish metapopulation}

approximately one week, after which their proportion diminishes considerably.

Tracers released on the Wellington South coast are rapidly entrained in the strong tidal currents and eddies that form around the many headlands in this area (Figures 4.6, 4.9). This leads a proportion of these tracers to be advected against the prevailing current in the strait, though once out of the strong tidal currents around Wellingtons south coast, these tracers are subject to the mean currents (Figure 4.9) and do not disperse further north in significant numbers. The largest contingent of tracers is advected eastward along the entrance of Wellington Harbour and towards the next large bay, Palliser Bay, where a number of them are retained. A portion of Wellington south coast tracers are also entrained into Wellington harbour by tidal currents and remain in the harbour for extended periods of time.

Wellington Harbour-sourced tracers follow similar dispersal routes to those released on Wellington's south coast. They either remain in the harbour or disperse out of the harbour (Figure 4.7), usually becoming entrained in eastward currents that carry them to Palliser Bay. From there a number of tracers disperse around Cape Palliser in the east and into the Pacific Ocean, while others are retained in Palliser Bay.

A proportion of tracers, released in and around the Marlborough Sounds, exit the sounds and find themselves subjected to the mean current, which pushes them southwards through the Cook Strait (Figure 4.8). A northward flowing mean current coming up the eastern side of New Zealand's South Island (a branch of the Southland current), leads to a change in direction of the particle mass, which flows eastward upon exiting Cook Strait. The tracers reach coastal waters of the North Island just eastwards of Wellington. A large number of passive tracers released in the sounds are also retained within sheltered embayments within the sounds. 


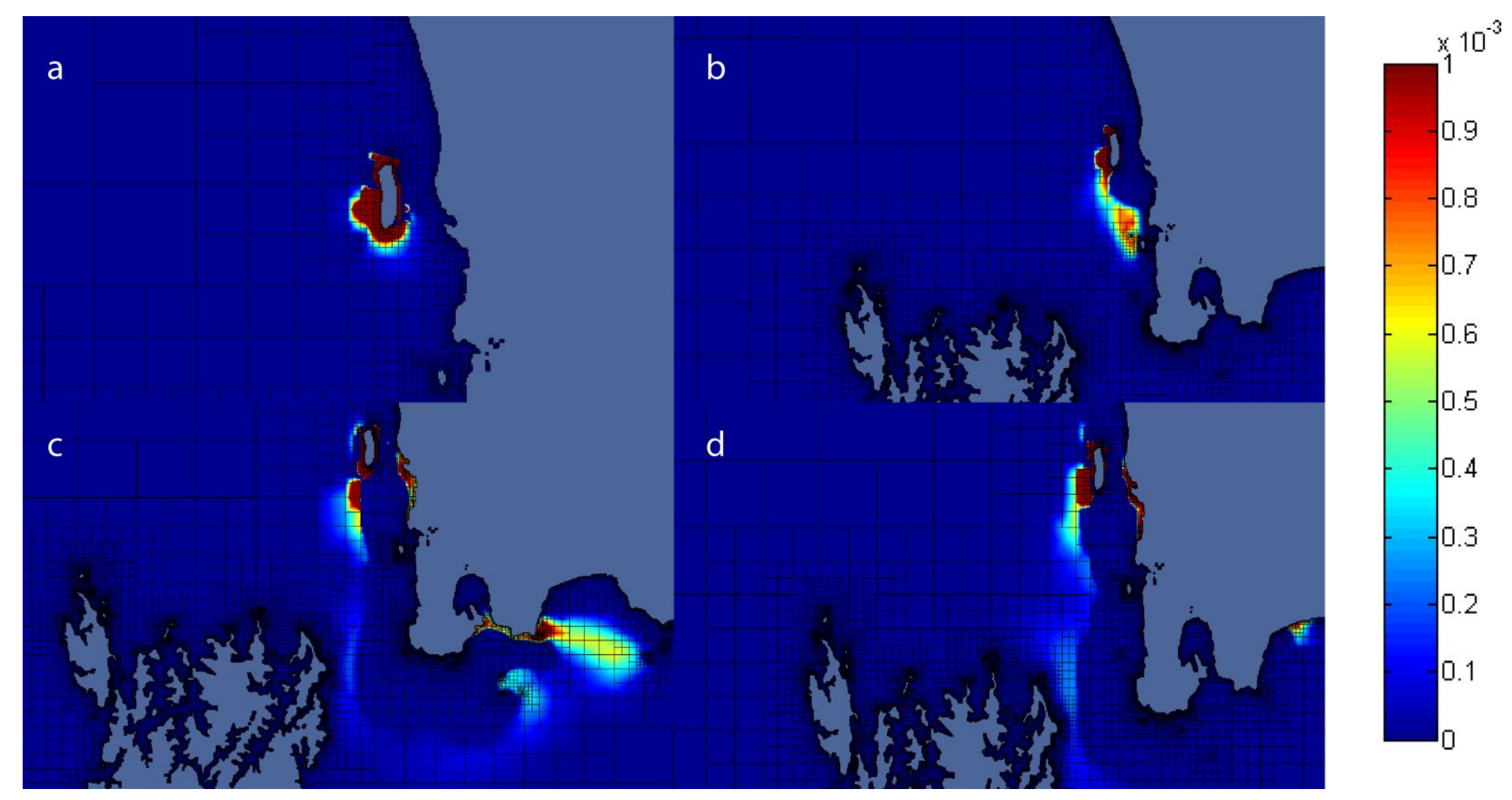

Figure 4.5: Tracer concentration of a single cohort released on Jan $2^{\text {nd }} 2008$ (red - T>0.001) a) $12 \mathrm{~h}$, b) $48 \mathrm{~h}, \mathrm{c}$ ) 7 days and d) 14 days after being released at sites around Kapiti Island. Release concentration was $\mathrm{T}=1$ in each square that corresponded to a depth between 3-5 metres. The black mesh indicates the level of refinement of the model. 


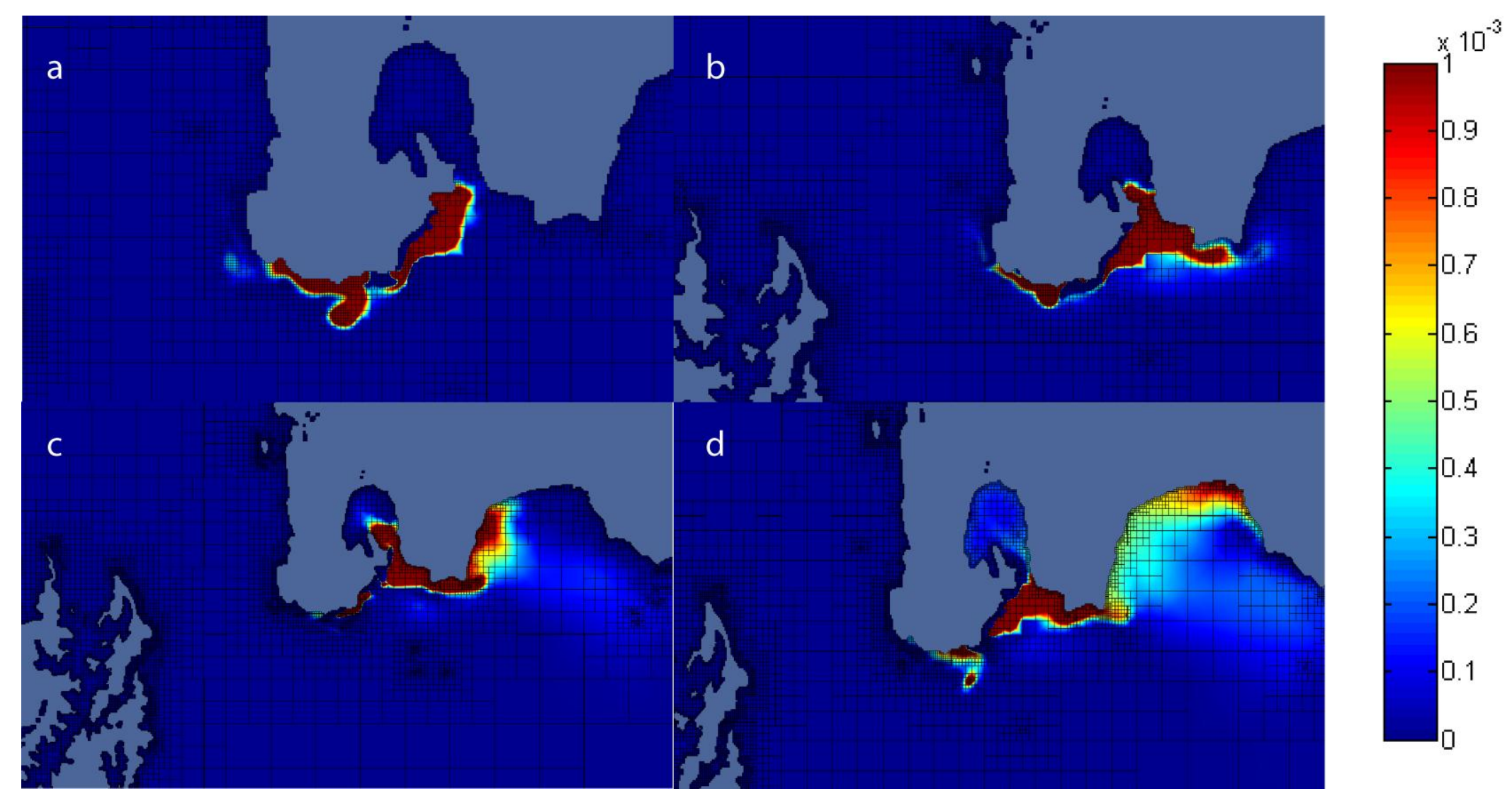

Figure 4.6: Tracer concentration of a single cohort released on Jan $2^{\text {nd }} 2008$ (red - T>0.001) a) $12 \mathrm{~h}$, b) $\left.48 \mathrm{~h}, \mathrm{c}\right) 7$ days and d) 14 days after being released at sites on Wellington's south coast. Release concentration was $T=1$ in each square that corresponded to a depth between 3-5 metres. The black mesh indicates the level of refinement of the model. 


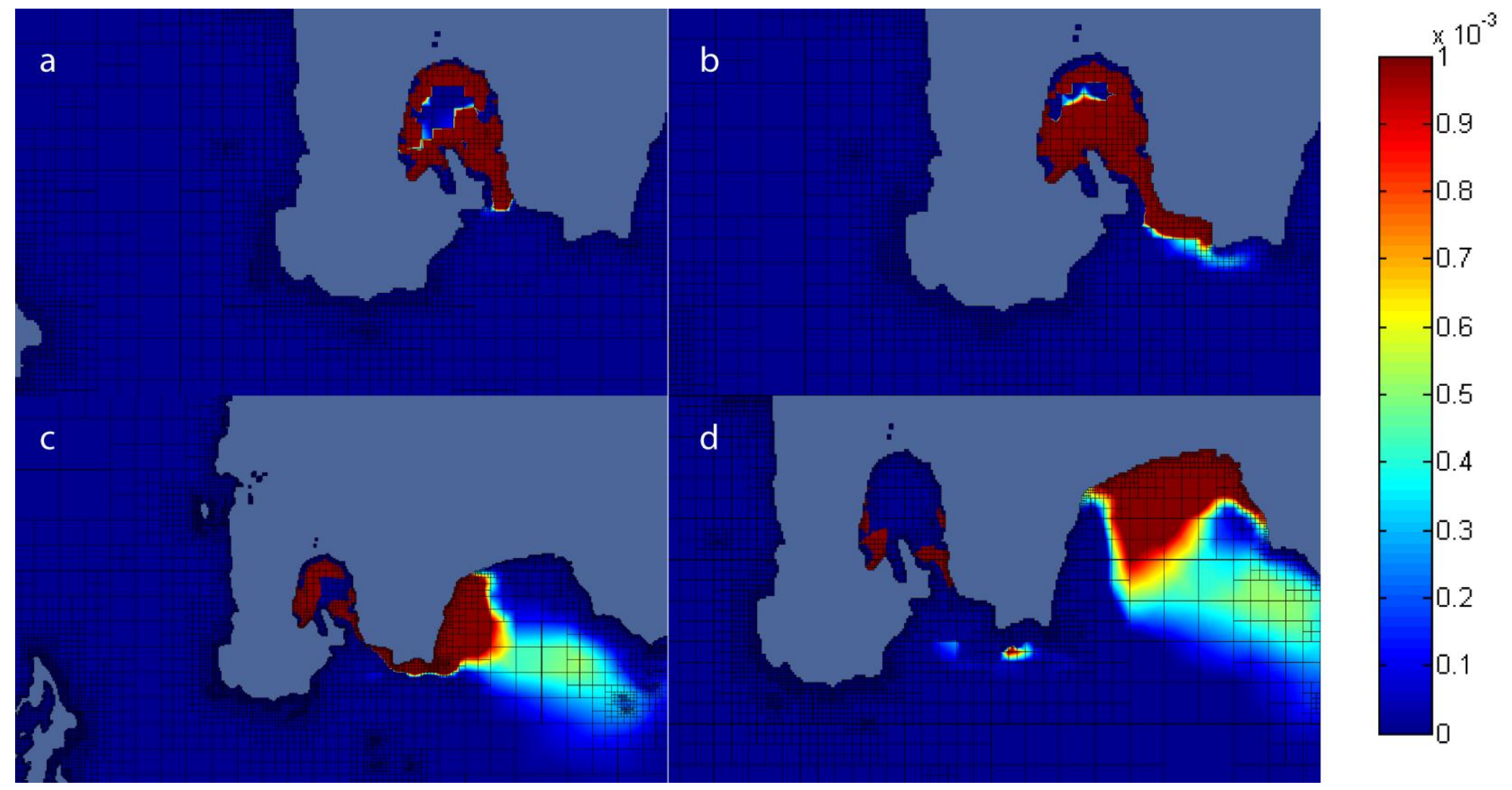

Figure 4.7: Tracers concentration of a single cohort released on Jan $2^{\text {nd }} 2008$ (red - T>0.001) a) $12 \mathrm{~h}$, b) $48 \mathrm{~h}, \mathrm{c}$ ) 7 days and d) 14 days after being released at sites in Wellington's harbour. Release concentration was $\mathrm{T}=1$ in each square that corresponded to a depth between 3-5 metres. The black mesh indicates the level of refinement of the model. 


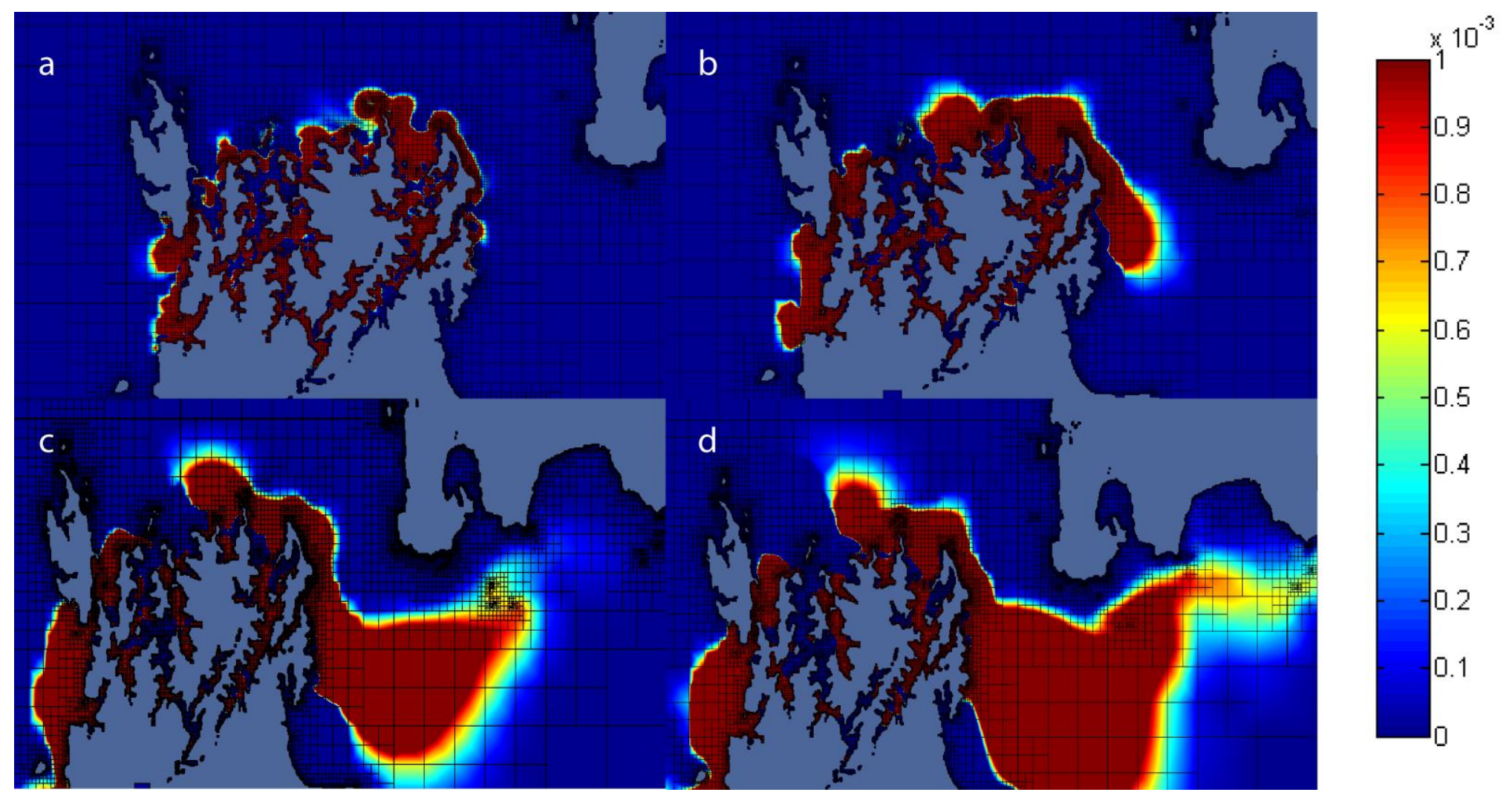

Figure 4.8: Tracers concentration of a single cohort released on Jan $2^{\text {nd }} 2008$ (red - T>0.001) a) $12 \mathrm{~h}, \mathrm{~b}$ ) $48 \mathrm{~h}, \mathrm{c}$ ) 7 days and d) 14 days after being released at sites in the Marlborough Sounds. Release concentration was $\mathrm{T}=1$ in each square that corresponded to a depth between 3-5 metres. The black mesh indicates the level of refinement of the model. 


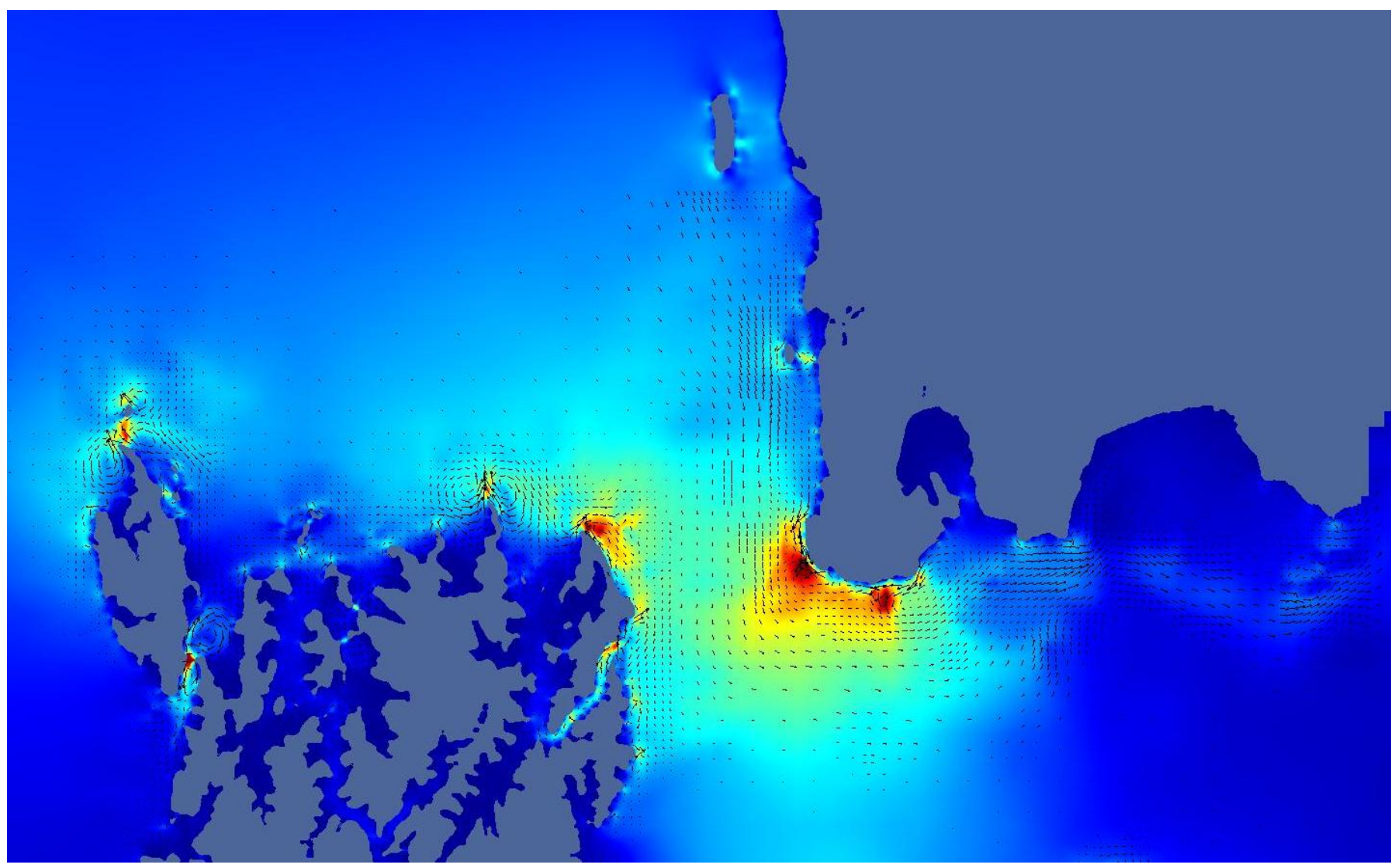

Figure 4.9: Mean ccurrent velocities and directions in Cook Strait as predicted by the Gerris flow solver ocean model for the period of the study. Colours and vectors are scaled to a maximum velocity of $1 \mathrm{~m} / \mathrm{s}(\mathrm{red})$ and displayed at a maximum of 8 refinement steps for visualization purposes. 


\section{Chapter 4. Larval dispersal pathways in a reef fish metapopulation}

The patterns described above were sustained over extended periods of time (and well beyond the week displayed in the figures), however at ever diminishing proportions due to a single release of tracers. Even after three weeks a small portion of tracers still leave the Marlborough sounds to travel towards Wellington. The same holds for Kapiti and Wellington. Similarly, a proportion of larvae remain retained at all sites for extended periods of time.

\subsubsection{Comparing otolith and simulation results}

Many of the connections found by otolith chemistry are supported by the tracer movements in the hydrodynamic model (Figure 4.10). Both, selfrecruitment and south-eastward dispersal of F. lapillum larvae are found empirically (Figure $4.3 \& 4.10$ ) and are evident from passive tracers (Figure 4.5-4.8). However, dispersal from Wellington to Kapiti Island is inferred by otolith chemistry but is not predicted by the passive dispersal model (Figure 4.10). While none of the dispersers at Long Island are assigned to Kapiti Island by the classification model, there is some, if little, probability mass associated with this dispersal pathway, which is supported by hydrodynamic simulations.

\subsection{Discussion}

Larval dispersal in the ocean is a notoriously complex phenomenon to describe both empirically and by simulation (Levin 2006, Cowen et al. 2007, Zimmer et al. 2009). Complex larval behaviours, potentially arising as evolutionary adaptations to local flow regimes or patchy habitat (Byers \& Pringle 2006, Baskett et al. 2007), make it difficult to appreciate the worth of simulation studies without any empirical verification. Similarly, results of dispersal obtained on ecological timescales are generally snapshots of connectivity in a system, and assuming generality of such snap-shots may lead to misconceptions about dispersal patterns in a system. The premise 


\subsection{Discussion}

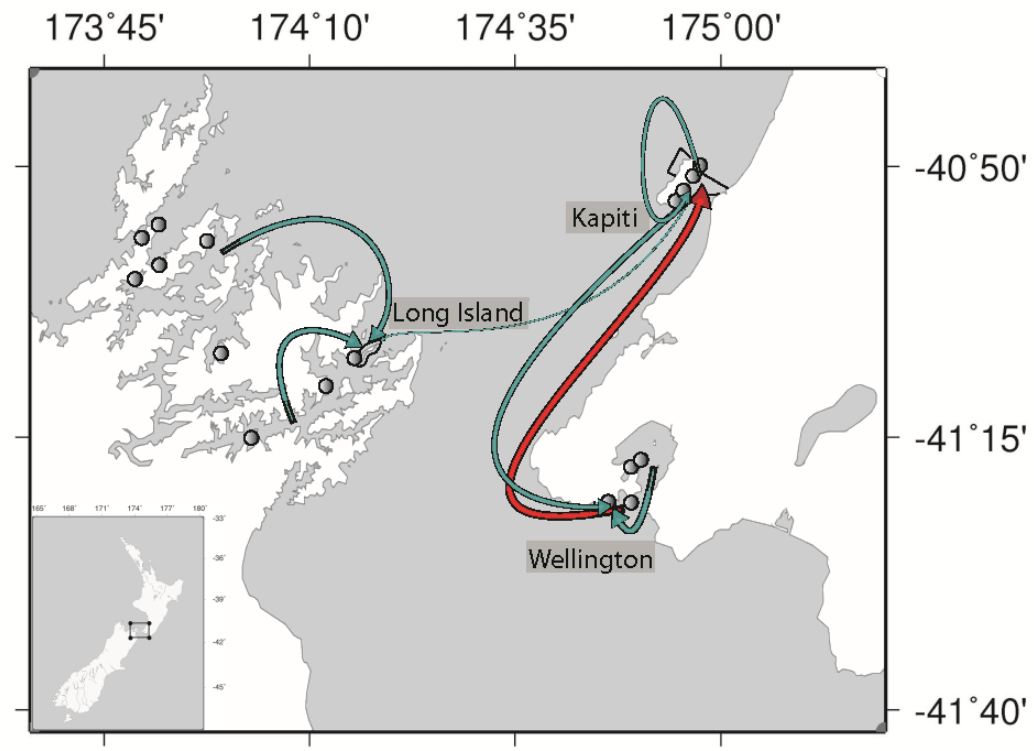

Figure 4.10: Dispersal pathways inferred by otolith chemistry (arrows). Blue arrows indicate support from the hydrodynamic simulations for these pathways; the red arrow depicts a pathway for which there is no evidence from the passive dispersal model. Arrow widths are proportional to the mean of the posterior distribution of inferred proportions of larvae dispersed along each pathway. Only connections with a posterior mean of $>0.05$ are displayed.

of this study was to compare a snap-shot of empirically observed connectivity with predictions about the dispersal of passive simulated larvae obtained from a fine scale hydrodynamic model in order to infer the existence of predicted pathways on an ecological time-scale and to gain mechanistic insight into the drivers of observed dispersal patterns in the system.

Empirical results suggest that regions are supplied by a mix of locally spawned larvae and larvae originating from distant sites. This is consistent with an emerging view that most local marine populations are sustained by a mixture of locally produced and distance-sourced recruits, albeit differences between species in larval durations and behaviours (e.g. Almany et al. 2007). The degree to which sites are supplied by selfrecruited or dispersed recruits is likely due to local physical conditions 


\section{Chapter 4. Larval dispersal pathways in a reef fish metapopulation}

interacting with larval behaviours. Shanks et al. (2003) suggest that there are essentially two modes of dispersal: larvae either disperse far and or hardly disperse at all, attributing the latter to behavioural adaptations (see also Shanks 2009). The hydrodynamic model provides a null hypothesis of passive dispersal (Shanks 2009) and results suggest that some of the patterns observed here may be due to passive mechanisms alone: Larvae are retained in all regions for extended periods of time throughout the simulation. This may be somewhat surprising in Kapiti, where strong alongshore currents develop (Chiswell \& Stevens 2010). Since F. lapillum deposit their eggs in very shallow water however, a loss of current speed close to shore may help larvae to be retained near spawning sites (Shanks \& Kipp Shearman 2009). The refined grid along the coast in the model employed here may be particularly useful in capturing this phenomenon, which may be difficult to reproduce with a fixed grid model.

It may be easier to appreciate that enclosed locations, such as Wellington Harbour, may passively retain larvae in enclosed bays away from the harbour mouth and strong tidal currents. Otolith chemistry provides some support of retention in enclosed bays: In the Marlborough Sounds recruit otolith chemistry suggests that regional self-recruitment is due to larvae spawned within the inner Queen Charlotte Sound while dispersed larvae seem to have come from sites towards the opening of upstream Pelorus sound. Self-recruitment may therefore be a rather prevalent mode of replenishment for many of the local populations in the strait. This is supported by recent research in the Wellington region using the same study species (Shima \& Swearer 2009b, Swearer \& Shima 2010) as well as by a recent genetic study which found significant differentiation between some populations in a prevalent intertidal snail in and around Cook Strait (Salinas de Leon et al. - in review). Such self-recruitment in the sites covered by this study is not necessarily associated with larval homing behaviour - it may be explained by passive mechanisms in enclosed and 


\subsection{Discussion}

shallow bays as well as in retentive eddies forming on complex coastlines (Mace \& Morgan 2006).

While self-seeding is evident for $F$. lapillum from both the hydrodynamic model and otolith chemistry, so is dispersal between regions and their marine reserves. Most connections inferred by otolith chemistry are readily explained by the prevailing current through Cook Strait: the D'Urville current flows through the Strait, moving water-masses in a southeasterly direction before encountering and mixing with the Southland current (Heath 1985). This is reflected in dispersal of tracers from Kapiti and the Marlborough Sounds in the hydrodynamic model which predicts both of these connections. Dispersal from the upper-Marlborough Sounds (i.e. Pelorus Sound) to Long Island and dispersal from Kapiti Island to Wellington can be attributed to dispersal of larvae within this mean current (Figure 4.10). Downstream connections thus seem to be prevalent and contribute recruits to populations in both Islands.

Dispersal evidenced empirically is restricted to within-Island exchange of larvae. The hydrodynamic model predicts that passive propagules from the Marlborough sounds should move towards the lower part of the North Island, but the sample size in this study may not be large enough to detect this connection if only a few larvae make or survive such a journey. Shima \& Swearer $(2009 a, 2010)$ found that dispersal out of inshore waters (i.e. Wellington Harbour) may lower a disperser's fitness and subsequent survival for F. lapillum. Fish may therefore be adapted to avoid such long distance dispersal by aiming to stay near high resource waters. Alternatively, few larvae may actually survive long periods in food depleted waters and or may have low fitness at arrival and thus do not survive for long post-settlement.

More intriguingly however, otolith chemistry indicates that dispersal may also happen against the prevailing current in this system (Figure 4.10). 


\section{Chapter 4. Larval dispersal pathways in a reef fish metapopulation}

While the hydrodynamic model shows that a proportion of tracers released in Wellington get swept upstream by tidal motion, there is no support from the model for these empirical results. However, Heath (1986) found a north-westward mean flow north-west of Wellington. I find that a fraction of passive tracers disperse in this direction before being captured and driven south-eastward by the mean flow in Cook Strait. Nevertheless, such conditions may be accentuated by strong southerly winds (Chiswell \& Stevens 2010) and could be responsible for transport of larvae against the overall mean current through the Strait. Theory predicts that selective pressure in advective environments will lead to adaptations to counter advection and loss of propagules (Byers \& Pringle 2006). The use of current variability has been predicted (Shanks \& Eckert 2005) and empirically shown to help near-shore species avoid advection in mean currents (Breitburg et al. 1995, Paris \& Cowen 2004, Morgan \& Fisher 2010) and may allow populations to be maintained at the upstream edge of species distributions (Byers \& Pringle 2006). Such variability, coupled with larval behaviour, could thus permit larvae to disperse against overall mean currents, and may have led to the patterns evidenced by otolith chemistry.

As Kapiti and Wellington have overlapping distributions of geochemical signatures (Figure 2.3, Chapter 2), an alternative explanation for larvae dispersing from Wellington to Kapiti could be that these larvae are simply being miss-assigned to Wellington. This seems unlikely however since it is the assignments to Kapiti that are predominantly associated with low classification probabilities. Most fish attributed to Wellington displayed high classification probabilities. Alternatively, some sources further upstream with a signature similar to that of Wellington Harbour may have contributed to recruits at Kapiti. This seems unlikely since model generated predictions and personal observations do not suggest that other local populations of substantial abundance and thus potential sources exist along this coastline. 


\subsection{Discussion}

A number of fish were attributed to sites other than the sampled ones within each region. In Wellington, for instance, fish that seemed to be selfrecruited did not associate with baseline samples. Post-hoc examination of this cluster revealed higher $\mathrm{P}$ and $\mathrm{S}$ concentrations. These fish may have been spawned near the river mouth of Hutt River, which enters Wellington Harbour. These sites were not sampled for eggs to construct the baseline due to high turbidity, but Hutt river discharge may explain these values. This is supported by comparatively high Ba values in the core which may be produced by a freshwater source (Hamilton \& Warner 2009).

This study demonstrates how empirical and simulation results can give complementary results which provide insight into dispersal mechanisms at play in a given system (see also Gilg \& Hilbish 2003, Nahas et al. 2003, Ashford et al. 2010): Hydrodynamic model predictions can provide a physical explanation of dispersal patterns found empirically in the system. Since retention and passive dispersal with prevailing currents seem to occur throughout the system, these may be prevalent dispersal modes. The hydrodynamic model also provides scrutiny for empirical results. I could not find evidence for dispersal between the two main Islands of New Zealand, though the model provides some evidence for such a connection: a more concentrated effort with higher sample sizes could help answer the question of whether this connection is ecologically relevant. The apparent upstream connection challenges the assumption of passive dispersal in the model and indicates that other factors may be at play: I may have missed an important contributing source for Kapiti Island, or some important component of dispersal, such as larval behaviour, is not represented by the model. I investigate these patterns further in chapter 6 .

Hydrodynamics can be a strong constraining force, dictating species distributions (Gaylord \& Gaines 2000) and dispersal patterns (Black \& Moran 1991, Gilg \& Hilbish 2003, Nahas et al. 2003), but they are rarely 
Chapter 4. Larval dispersal pathways in a reef fish metapopulation

sufficient to account for measured dispersal patterns (Shanks 2009). Dispersal of larvae involves a large number of factors, and the complexity of this phenomenon is unlikely to be described by a single factor and can thus not be addressed adequately by any single approach. Combining approaches that bridge time-scales and complement inadequacies inherent in any single approach is essential to describe connectivity in a system (Bradbury et al. 2008). 
4.4 Discussion 


\section{Chapter 5}

\section{Plasticity and similarity in dispersal histories: a Bayesian framework for characterizing fish dispersal from otolith chemistry profiles}

\subsection{Introduction}

Studying dispersal and migration of larvae and adult animals in the ocean is essential to understanding population dynamics (Armsworth 2002, Gaines et al. 2007) and managing exploited and endangered species (Fogarty \& Botsford 2007). Tracking migrations of marine animals is difficult, especially in species that potentially disperse great distances and/or remain hidden from direct observation. In fishes, analyses of trace elements within otoliths (ear-stones) have helped expose life history characteristics long hidden from direct observation (Campana \& Thorrold 2001, Elsdon et al. 2008). Otoliths have, for instance, been used to infer self-recruitment in reef fish (Swearer et al. 1999), natal homing of spawning adults to distinct spawning grounds (Thorrold et al. 2001) and identities of migratory contingents (Secor \& Piccoli 1996, Miller et al. 2010). In larval fish, such analyses suggest that different dispersal patterns may affect larval condition and subsequent juvenile survival (Shima \& Swearer 2009a, 2010). 


\subsection{Introduction}

Inferences about fish dispersal made from analyses of otolith chemical analyses are based on the incorporation of chemical trace elements into the otolith calcium carbonate matrix: the otolith grows concentrically and continuously incorporates trace elements from the surrounding environment. Although not always experimentally verified, elemental concentrations within the otolith are often assumed to reflect the surrounding environment (Elsdon et al. 2008). The concentric growth pattern of otoliths, in conjunction with high resolution sampling techniques (i.e. Laser Ablation Inductively-Coupled Plasma Mass Spectroscopy (LAICPMS)) makes it possible to study migratory patterns in fish by taking multiple measurements of elements of interest along the growth axis of an otolith - this is usually referred to as a transect or profile of otolith chemistry, which is made up of individual chemical measurements or 'scans' (i.e. individual laser ablation pits). Many recent studies use such profiles along the major otolith growth axis to identify fish movements amongst chemically distinct water-masses or to differentiate between groups of fish with similar migratory or dispersal histories (often called migratory 'contingents' (Secor 1999)) (e.g. Secor \& Piccoli 1996, Elsdon \& Gillanders 2003, Fablet et al. 2007, Elsdon et al. 2008). Some studies are based on information about water chemistry and its influence on otolith chemical composition for a particular species (e.g. Elsdon \& Gillanders 2003, Miller et al. 2010). Other studies are based on a set of underlying hypotheses about environmental influences on otolith chemistry, usually drawing on a body of literature about such effects (Hamilton et al. 2008, Shima \& Swearer 2009a, Berumen et al. 2010).

A number of different chemical elements and statistical approaches have been used to infer dispersal histories and associated processes. Fablet et al. (2007) reviewed a number of studies based on profiles of Sr:Ca as indicators of freshwater residence. They found that many such studies did not adequately address the statistical issues inherent in chemical profile 
data. Many studies rely on visual or ad-hoc characterization of these time series. Furthermore, measurements (i.e. individual scans or binned means) along a chemical profile cannot be thought of as independent for a single fish, and ignoring such non-independence can be problematic.

Elsdon et al (2008) proposed to use repeated measures ANOVA (RMANOVA) or its mixed model extension as a framework to test for differences in groups of fish over time, and this has by far been the most prevalent method of analysis for this type of data. While RM-ANOVA provides some desirable features - one may for instance test the influence of water chemistry or age on observed differences - it also has substantial drawbacks. For instance, the ecological meaning of a significant difference between chemical profiles is not intuitively clear: a result of statistically significant differences in profiles tells little about the nature of these differences. Pairwise differences between scans in the profile can be used (Berumen et al. 2010), but this becomes cumbersome with high resolution transects and a decent number of samples. Furthermore, one may wish to analyse time series of variable length to account for age differences in fish. This makes the application and interpretation of (mixed model) repeated measures ANOVA difficult. Other approaches have been proposed (Sandin et al. 2005, Hedger et al. 2008, Shima \& Swearer 2009a), but of these methods only the approach of Hedger et al. provides time-resolved information about dispersal patterns. Other methods find ways to characterize contingents but do not provide other relevant descriptions of dispersal or migration history.

Fablet et al. (2007) realized the need for a statistical framework which effectively addresses the shortcomings in previous analyses and proposed a sequential analysis of otolith chemical profiles based on latent state models. These 'latent states' are in fact the hidden environments or watermasses through which fish disperse. Their study is the first to construct 


\subsection{Methods}

generative probabilistic models from hypotheses or knowledge of processes that lead to measured profiles. For instance, if fish are thought to move from estuarine to marine environments at different ages or with different probabilities one may construct a model which reflects the differences in otolith chemistry that are produced by each environment and which explicitly models parameters of interest, such as residence times in each environment or probabilities of movement between environments.

While their paper is an important step towards a statistically rigorous treatment of such chemical profiles and associated ecological parameters of interest, Fablet et al. (2007) stop short of a general framework. Furthermore, although stated as a Bayesian analysis, their sequential analysis does not 'propagate' uncertainty, and their results are (maximum likelihood) point estimates rather than Bayesian posterior distributions (their paper is Bayesian from the use of Bayes theorem to compute the most likely sequence of states for each fish. The inference presented is, however, not Bayesian). In this paper I build on the general idea of a probabilistic framework for modelling otolith profiles. I propose a general, fully Bayesian approach using latent state models, which I suggest as a flexible and powerful framework for analysing fish dispersal. To show how this framework can be used to accommodate and test a number of relevant ecological hypotheses, I first use a simple mixture model from which it is possible to infer residence and transition between environments, as well as test the influence of external parameters (i.e. age) on inferred dispersal histories. I then propose two measures of similarity of dispersal histories which provide a new method to characterize migratory contingents. An accompanying visualization approach allows one to gain further insight into the nature of dispersal histories of inferred contingents. I lastly discuss how different approaches to model selection can help find appropriate models for a given dataset. 


\subsection{Methods}

\subsubsection{Generative models for chemical transects}

It is often possible to make predictions about the influence of certain water-masses on otolith chemistry, regardless of whether prior knowledge or hypotheses constructed from known patterns are used. To formally construct such a generative model for time series of chemical measures across otoliths, the generative hypothesis needs to be expressed in probabilistic terms. Coding as $\boldsymbol{y}_{\boldsymbol{i}}=y_{i, 1}, \ldots, y_{i, t}, \ldots, y_{i, T}$ the $T$ measurements made across the otolith of fish $i$ (other parameters are indexed accordingly), the time series can be written as a mixture of $K$ different environmental (or water-mass) signatures which fish $i$ $(i=1, \ldots, N)$ experienced. The joint density of $\boldsymbol{y}=\left(\boldsymbol{y}_{\mathbf{1}} \ldots \boldsymbol{y}_{i} \ldots \boldsymbol{y}_{\boldsymbol{N}}\right)$ can then be written as:

$$
f(\boldsymbol{y} \mid \boldsymbol{\pi}, \theta)=\prod_{i=1}^{N} \prod_{t=1}^{T} \sum_{k=1}^{K} \pi_{i, k} f\left(y_{i, t} \mid \theta_{k}\right)
$$

where $f\left(y_{i, t} \mid \theta_{k}\right)$ is the probability density of the distribution (parameterized by $\theta_{k}$ ) associated with environment $k$ evaluated at $y_{i, t}$. A proportion $\pi_{i, k}$ of the signatures along the profile of individual $i$ are generated by environment $k$, reflecting residency in this environment for this proportion of time. $\boldsymbol{\pi}_{\boldsymbol{i}}=\left(\pi_{i}, 1 \ldots \pi_{i}, k \ldots \pi_{i}, K\right)$ and $\boldsymbol{\pi}=\left(\pi_{1} \ldots \pi_{i} \ldots \pi_{N}\right)$ then denote the vector over all source proportions for individual $i$, and the set of vectors of source proportions for all individuals, respectively. It is commonly assumed that different chemical measures pertaining to a given environment experienced by a fish follow a (multivariate) normal distribution, such that $\theta_{k}=\left\{\mu_{k}, \Sigma_{k}\right\}$, with $\theta=\left(\theta_{1} \ldots \theta_{k} \ldots \theta_{K}\right)$ for the $K$ sources, although other continuous distributions may be used. This model assumes independence in measurements along the otolith profile, a property which I discuss further on. 

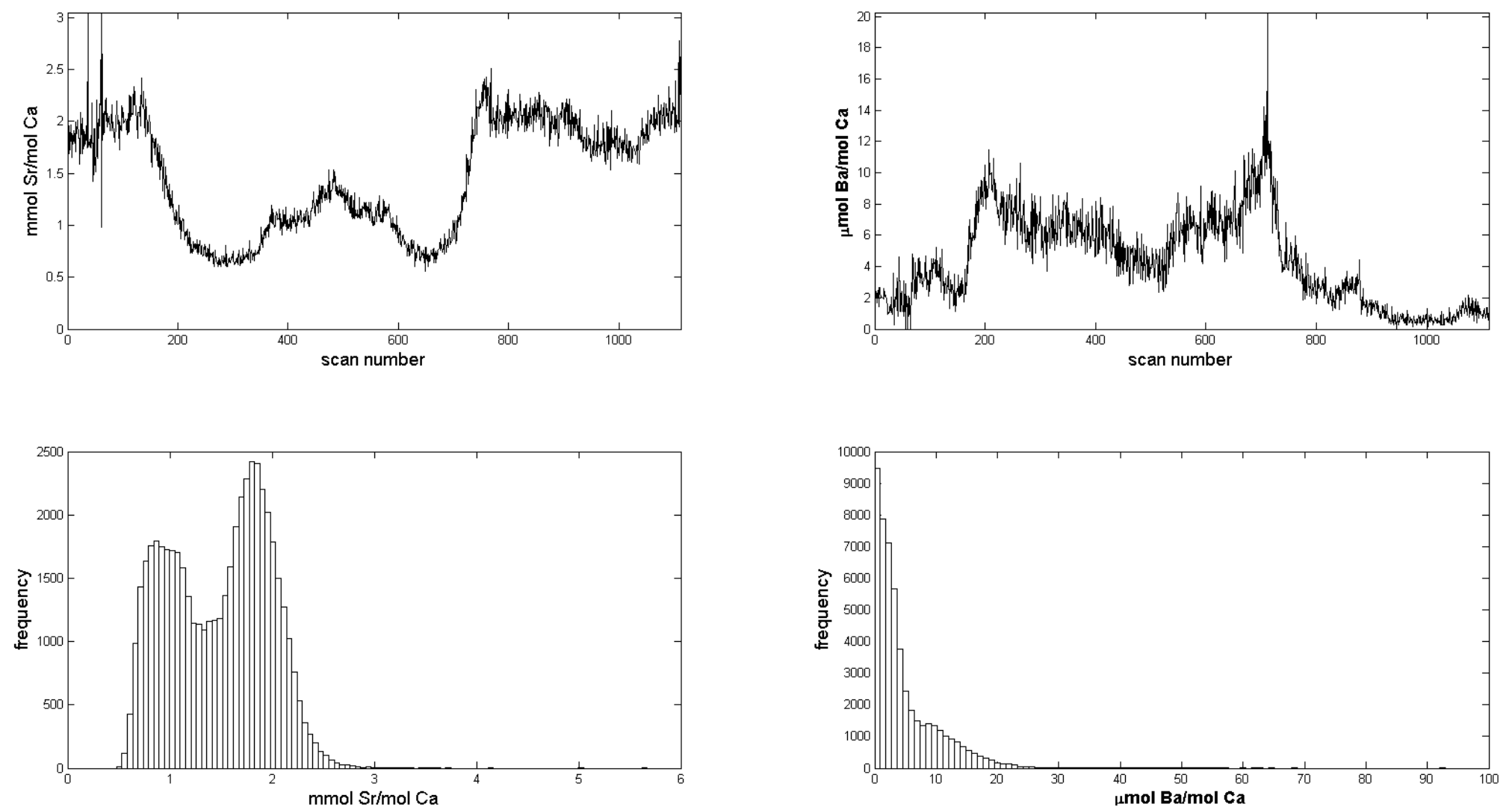

Figure 5.1: Upper panels: Example profiles for Strontium and Barium across Chinook salmon otoliths. Lower panels: Histograms of count frequency for Strontium and Barium measures for all N Chinook salmon from Miller et al (2010). 
Plotting the frequency distribution of individual scans along some transects of Chinook salmon data used later on in this chapter illustrates how a mixture model makes intuitive sense (Figure 5.1 ). This mixture model is also the basic model proposed by Fablet et al. (2007) for a mixture of three environmental signatures (marine, estuarine and river) found along the profiles of European eels in the Gironde watershed, France. As shown in their study, maximum likelihood estimates of model parameters can be readily obtained from the EM algorithm. They also proposed a first extension of this model which allows testing for the effects of explanatory variables on the observed profiles. The model can in fact be readily adapted and extended to accommodate and test a variety of hypotheses. Illustrating how this can be achieved will constitute the remainder of this paper. To this end, I first write an equivalent Bayesian hierarchical version of equation (1) which helps to clarify conditional dependency relationships within the model. These relationships are visualized using directed acyclic graphs, which depict a model structure in terms of conditional dependences (Gilks et al. 1996). A hierarchical formulation can be written as

$$
\begin{aligned}
y_{i, t} \mid \theta_{\xi_{i, t}}, \xi_{i, t} & \sim F\left(\theta_{\xi_{i, t}}\right) \\
\xi_{i, t} \mid \boldsymbol{\pi}_{\boldsymbol{i}} & \sim \operatorname{Multinomial}\left(1, \boldsymbol{\pi}_{\boldsymbol{i}}\right) \\
\theta_{k} & \sim G_{0} \\
\boldsymbol{\pi}_{\boldsymbol{i}} & \sim \operatorname{Dirichlet}\left(\gamma_{1}, \ldots, \gamma_{K}\right)
\end{aligned}
$$

where the tilde $(\sim)$ means 'is distributed as'. $\xi_{i, t}$, such that $p_{i, k}=p\left(\xi_{i, t}=k\right)$ is a hidden (latent) state variable (i.e. environment/water-mass) which relates observation $t$ of individual $i$ to one of the $K$ environments and takes discrete values (Figure 5.2a, option 1). $G_{0}$ is a generic term for the prior of the mixture distribution parameters (i.e. the prior for the mean and variance parameters $\{\mu, \Sigma\}$ in $\theta$ ). Only the last two rows of this hierarchy are specific to the Bayesian analysis of the mixture model as they 


\subsection{Methods}

specify prior distributions on hyper-parameters. Note also that the multinomial distribution collapses to a binomial one if only two environments are of interest. The generative model can be expressed as follows: Fish $i$ experiences one of $K$ water-masses at time $t$. This watermass is drawn from a distribution of possible environments with probability $\pi_{i, k}$. The observed signature is then generated from the distribution (i.e. $N\left(\theta_{k}\right)$ ) associated with this environment.

This model can be readily extended to test for the effects of covariates $x$ (i.e. age, temperature) on observed transects by adding a line to the hierarchical formulation, such that

$$
\mu \mid x, a, b, \Sigma \sim N\left(a+b x, \Sigma_{\mu}\right) .
$$

This setup specifies a simple regression relationship (Figure 5.2a, option 2) for the parameter $\mu$. Often it is the sequence of environments visited or the use of environments that is of primary interest (Fablet et al. 2007, Shima \& Swearer 2009a, 2010). These parameters are estimated as $\xi_{i}$ and $\pi$ of the model. Covariates can be included in a similar way to model some effect on the parameter $\pi$. An older fish, for instance, may be more likely to have visited a spawning ground than a juvenile. One could thus relate $\pi$ to the age of the fish by using a prior for individual $\gamma$, the prior 'counts' for $\pi$. 
a)

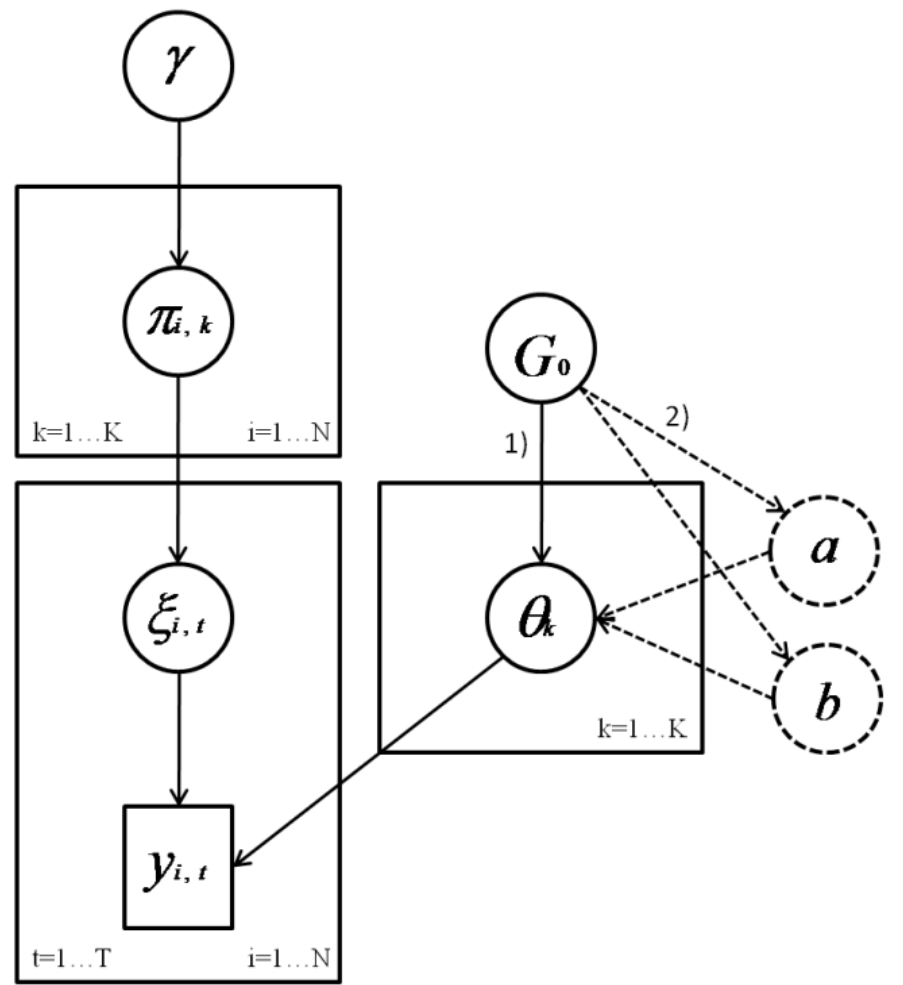

b)

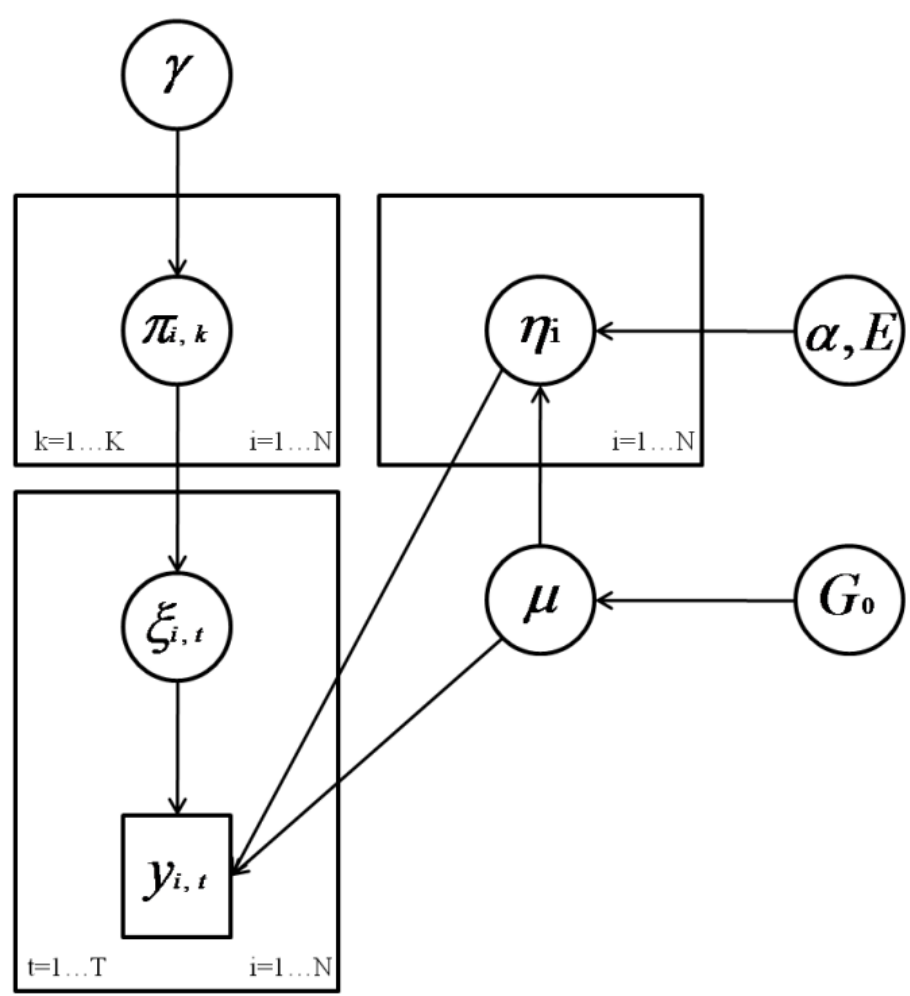

Figure 5.2: Directed acyclic graphs of a) the simple mixture model for chemical transects across otoliths 1 ) without covariates and 2) with testing for influence of covariates, and b) the random effects mixture model. Conditional independence is visualized by graph structure: descendants (defined by the direction of the arrow) of any node (individual parameters or sets thereof) in the graph are conditionally independent of that nodes parents given the node's value. Deterministic nodes are square (here only the measured data), stochastic nodes are round. Embedding 'plates' (squares surrounding sets of nodes) indicate that parameters are estimated for each element of the plate (i.e. all $i=1 \ldots N$ fish and $t=1 \ldots T$ measurements for each fish). 


\subsubsection{The Bayesian approach: Prior knowledge and the lack thereof}

The remainder of this chapter is based on the Bayesian treatment of the general model outlined in equation (5.1). There are multiple reasons for this. I already mentioned that the Bayesian approach can accurately reflect uncertainty about all parameters in the model. Another advantage is that the quantities I will use below to define similarity of profiles are readily available from a Bayesian treatment of the model.

Encoding prior information can be helpful in analysing chemical profiles, and this information is a fundamental quantity in Bayesian analysis. Studies which experimentally relate environmental factors to otolith chemistry can for instance be used to construct prior distributions for environments, which in turn can facilitate estimation of dispersal histories and other parameters. I illustrate this with the Chinook salmon example later on. Similarly, information about the effects of age or growth on the incorporation of chemicals into the otolith may be available. This information could be encoded as priors on the above regression formula.

Bayesian approaches are sometimes criticized for the fact that the prior probability is part of the analysis, even when no substantial information exists. Often, it is possible to find formulations for prior distributions which convey minimal information (so-called vague priors). Such vaguely informative priors however can lead to problems with estimation of latent state models: the labels that are given to the environments become meaningless in the model and $\xi$ will change 'identity' throughout the estimation of this model - the so-called 'label switching' problem. A simple but effective way to re-parameterize the model is by expressing the means of different environments in an additive fashion, such that $\mu_{2}=\mu_{1}+\tau$ and restricting tau to be positive. Such problems can also be dealt with by using informative priors for the parameters of different mixture components (i.e. one might suspect that values of some chemicals are higher in rivers than the 


\section{Chapter 5. A Bayesian framework for characterizing fish dispersal}

ocean or vice versa), which therefore make the model formulation and estimation somewhat easier.

\subsubsection{Encoding alternate hypotheses}

Testing for covariate effects is but one application of the above model. I now illustrate how this model can be used to encode alternate hypotheses - or the lack of strong hypotheses. For example, there may be considerable variability in the signatures of an environment, for estuarine species for instance, substantial variation in salinity within an estuary may lead to variability in Sr signatures depending on which part of the estuary a fish resided in. Assuming that the environmental signatures are normally distributed, a natural extension is then a hierarchical model for individual environmental means (a random effects model). In the next chapter I use this model for reef fish recruits to estimate nearshore residency of these fish during their larval period. In that case there are thought to be multiple larval origins along a coast that exhibit variability in the distribution of signatures among nearshore spawning habitats. Thus, one may not have just one distribution for larvae spawned and residing nearshore, but rather a number of them which are linked via common prior. This model can be encoded by modifying the first line of the above model to read

$$
\begin{aligned}
y_{i, t} \mid \theta_{\xi_{i, t}}, \xi_{i, t} & \sim N\left(\mu+\xi_{i, t} \eta_{i}, \Sigma_{\xi_{i, t}}\right) \\
\eta_{i} \mid \alpha, \varsigma & \sim N(\alpha, \varsigma)
\end{aligned}
$$

$\theta$ now includes only the mean and variance of the water-mass(es) without a random effect. All other lines are identical to the original model (Figure 2b). Note that the means have an additive parameterization. $\eta_{i}$ can now be restricted to be positive (or give an informative prior) to ensure identifiability of the mixture. $\xi_{i, t}$ is 1 if a nearshore environment is drawn and zero otherwise, and the random effect is thus only used for nearshore means $\eta_{i}$. The nearshore characteristic (or random effects) distribution, from which individual nearshore means are drawn, is thus found at a lower level of the hierarchy. The above formulation suggests that variances of water-masses may be different, but one may choose to model 


\subsection{Methods}

variances of different water-masses as constant (this raises the question of how one actually defines a water-mass or environment, which I will discuss below).

This model may also be useful if fish migrate from the ocean to different tributaries in an estuary or a river system or vice versa. Variations on this setup would include random effects on means of each environment: fish may move from different rivers to different estuaries and end up in a mixed stock in the ocean. Flexibility can be added for both environments, provided that it is possible to distinguish between rivers and estuaries globally. The ease with which such hierarchical models can be implemented in a Bayesian setting is an additional argument in favour for adopting a Bayesian approach (Clark 2003).

A further hypothesis would be that most fish display variations of the same migratory behaviour, and are thus expected to spend related amounts of time in a given environment. Two options come to mind: one may use a common mixture distribution $\pi$ for all transects, or alternatively place a hierarchical prior on $\pi$. The former assumes that all fish share the same behaviour parameterized by $\pi$, while the latter assumes that each fish performs a variation of an inert behaviour. Both may be valid assumptions, and both allow information about behaviours to be shared between individual time-series. However, the hierarchical option is considerably more flexible. Rather than specifying the prior $\gamma_{1}, \ldots, \gamma_{K}$ for $\pi_{i, K}$ independently for each fish $\left(\gamma_{1}, \ldots, \gamma_{K}=1\right.$ is, for instance, often used as a 'vague' prior, see below), one may specify a common prior for the mixing proportions, i.e. $\gamma_{k} \sim \operatorname{Gamma}(u, v)$.

5.2.4 Estimating the number of (distinguishable) environments along a transect

A further motivation for a Bayesian analysis of dispersal comes from the possibility to actually estimate the number of distinct environments a fish may have experienced (see also chapter 3). In a dispersal study it may be of interest to know how many distinct (or distinguishable) water masses are reflected on the profiles of the sampled fish. The Bayesian approach allows us to place a prior on 
this number, which is termed the cardinality of the model. The specific form of the prior depends on the model, but in the mixture model setting this amounts to replacing the mixing distribution $\pi$ over mixture indicators $\xi$ with a draw from the Dirichlet Process (DP). The DP is often described as a distribution over distributions (Neal 2000): from a specified 'base' distribution (i.e. a normal distribution) it allows inference about the number and characteristics of such distributions in a dataset.

The DP can be used in two ways, the simpler of which is with the basic model in equation (1) if one does not want to specify the number of environments a priori. There may rarely be enough information content in a dataset to allow reliable inference of all parameters in this case. A more sophisticated application comes as a substitute for the individual random effect. This involves a mixture density as a random effect: the normal distribution is replaced by a mixture of normal distributions for instance. A DP prior is then used to infer the number of distinct environments at this level in the model. As Olsen et al. (2007) pointed out, the DP random effect is conceptually between an individual effects model for every subject and the random effects model, where individual effects are drawn from a single underlying distribution. With the DP, individuals are clustered into groups of similar characteristic. A thorough treatment of the DP and its use as a random effect is beyond the scope of this paper and the interested reader is referred to Olsen et al. (2007). Formally, the DP mixture model for transects can be written as

$$
\begin{aligned}
y_{i, t} \mid \theta_{i, t} & \sim F\left(\theta_{i, t}\right) \\
\theta_{i, t} & \sim G \\
G & \sim D P\left(\gamma, G_{0}\right)
\end{aligned}
$$

This model introduces a clustering of $\theta s$, such that some of the $\theta s$ will be identical amongst different fish, thereby specifying that these fish occupied the same watermass out of an infinite possibility of possible water masses. 


\subsection{Methods}

Coming back to DP random effects, it may be preferable to place the DP prior at a lower level of the hierarchy. This echoes the belief that an unknown number of distinct sources in one environment contribute to the observed fish sample. For instance, instead of trying to infer the total number of environments, we may wish to infer the number of nearshore water-masses that contribute to a larval pool, or the number of nurseries that contribute to a population (or stock) (i.e. salmon, snapper (Fowler et al. 2005)).

The random effects model can be modified accordingly, placing the DP prior on the random effect:

$$
\begin{aligned}
y_{i, t} \mid \mu, \eta_{i}, \xi_{i, t} & \sim N\left(\mu+\xi_{i, t} \eta_{i}, \Sigma_{\xi_{i, t}}\right) \\
\mu, \Sigma_{\mu} & \sim G_{0} \\
\eta_{i} \mid F & \sim F \\
F & \sim D P\left(\gamma, F_{0}\right)
\end{aligned}
$$

In this setup, some fish will experience the same random effect $\eta$ (i.e. the same river or natal estuary) and a distribution over the number of distinct environments can be inferred at this level by looking at the number of clusters in the posterior distribution of the model. As I will show in a simulation experiment, this can be very fruitful in multivariate situations, when distinctions between environments are not immediately obvious.

\subsubsection{Temporal dynamics}

The above models imply that observations are independent conditional on the environment experienced (the corresponding acyclic graph (Figure 5.2) show this clearly). However, states are also modelled independently at each time point (each scan or bin). As Fablet et al. (2007) remarked, these mixtures do not explicitly model movements, but rather model environments as the most likely point of residence at any time point. To model movements, these authors introduce a hidden Markov model (HMM, also called Markov switching models or Markov mixtures). A HMM is a mixture model for which the mixing distribution $\pi_{i, k}$ has been replaced by a Markov chain which models the underlying state dynamically 


\section{Chapter 5. A Bayesian framework for characterizing fish dispersal}

(Scott 2002). In a Markov chain the probability of being in state $\xi$ at time $t$ depends on the state at time $t-1$, and transition between states is governed by a transition matrix (Figure 5.3). This can be written as written as $p\left(\xi_{i, t}=k \mid \xi_{i, t-1}=j\right)=\pi_{k \mid j^{\prime}}^{i}$ where $\pi$ is now a matrix of one-step transition probabilities.

Modelling the state dynamics explicitly can have an important consequence, in that isolated spikes in readings along a profile are not necessarily attributed to movements between environments (Fablet et al. 2007). This phenomenon is usually termed 'filtering' in statistical and engineering jargon. To illustrate the difference between the mixture model and the HMM, consider a simple example of a group of fish, each moving once between two chemically distinct environments. The parameter $\pi$ in the simple mixture model has a natural interpretation of proportion of time spent in each environment. Assume each fish spends equal proportions of time in each environment. A point estimate (i.e. the mean of the posterior distribution) of $\pi$ in the mixture model would then (hopefully) be around 0.5 . Assuming that otolith growth rate is constant or that it is possible to obtain growth patterns from otolith structural analyses, the proportion $\pi$ is easily transformed to a measure of how much time fish spend in a particular environment by multiplying $\pi$ times the total time covered by the profile (i.e. the PLD or age of the fish).

The transition matrix, however, encodes the probability of movement between environments, and the above interpretations cannot be readily recovered from the transition matrix itself. An estimate of a transition matrix in the above example above would give a low probability to transitions since only one transition occurs for each fish. For 51 scans of otolith chemistry along a profile for a single fish the estimated value would thus be around 0.02 for a transition versus 0.98 for resident behaviour (since there are 50 possible transitions for 51 scans). An estimate of transition probabilities for only eleven scans would be around 0.1 for a transition and 0.9 for resident behaviour. The estimates of the transition matrix are thus not invariant to the sampling frequency and the number of scans. 


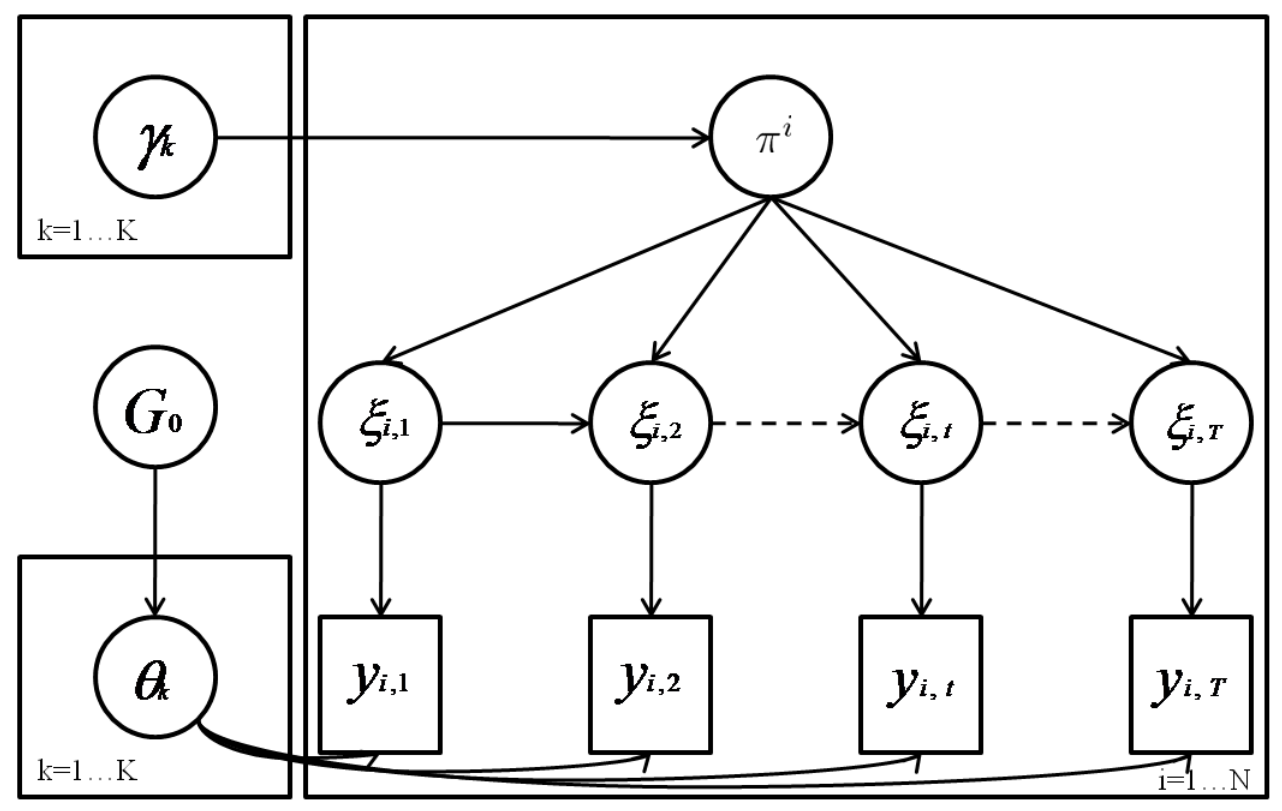

Figure 5.3: A hidden Markov model with individual transition matrices $\pi$ for each fish $\mathrm{i}$, which are governed by a single prior $\gamma$. Note that observations are still conditionally independent given the underlying sequence of states (i.e. environments), but the underlying states are now temporally dependent.

From the hierarchical formulation of the mixture model it is evident that $\pi$ can be thought of as a prior for the state identifiers $\xi$. Given the difference in numerical values between this parameter in the mixture versus the HMM, it is evident that the HMM places considerably more weight on the temporally explicit prior. This is the basis for 'filtering' in HMMs, where for some observations the prior outweighs the likelihood and some peaks in the data may be interpreted as noise rather than state transitions if these are a priori unlikely. As I will show below, filtering can become a nuisance when parameters are difficult to estimate, resulting in poor estimates for movement patterns.

Modelling the transition matrix can be dependent on further hypotheses. Fablet et al. (2007) use a single transition matrix for all fish. The underlying assumption is thus, as discussed above in the context of the mixing distribution, 


\section{Chapter 5. A Bayesian framework for characterizing fish dispersal}

that all fish have the same a priori propensity to move between a set of environments. One may instead wish to estimate transitions independently for each fish, or to give the prior for the transition matrix a hierarchical structure. The former may be difficult if there are few observations of transitions in each timeseries, whereas the hierarchical approach allows for information about transitions to be shared across profiles: a common 'inert' behaviour is thus assumed. Since the Dirichlet distribution is commonly used as a prior for each line of the transition matrix (Cappé et al. 2005), the same prior as for the hierarchical formulation of mixing distributions may be used. Another alternative is to model a single (common for all fish), time dependent transition matrix which captures changing propensities of the sampled fish to move between environments. The Markov Chain is then said to be non-homogenous. Care must be taken in this case that all fish are aligned such that individual scans correspond to the same age.

Apart from the Dirichlet process mixture, all of the mixture models developed in previous sections can be readily transformed into an HMM (though the Dirichlet random effects models can be used with an HMM). HMMs are, however, somewhat harder to sample from: the time dependence in the state sequence renders standard methods of sampling often ineffective. This is discussed in more detail along with Markov Chain Monte Carlo methods in Appendix 3.

\subsubsection{Estimating unknowns: MCMC and WinBUGS}

Bayesian approaches can have a drawback in that estimation of unknowns in the model is difficult and necessitates approximate inference algorithms such as Markov Chain Monte Carlo and related techniques. I present Gibbs sampling algorithms for the models I use in the next section in Appendices $A 2 \& A 3$, where I also discuss some issues encountered specifically with time explicit models discussed below. Note that for some of the simple models discussed here, inference can be straightforward with the freely available Bayesian model building software WinBUGS (Lunn et al. 2000). I provide some sample code for Winbugs implementation for simple mixture models in the appendix A4. Dirichlet Processes 


\subsection{Methods}

models (see below) can only be approximated by this software and the interested reader is referred to Ohlsen et al. (2007) for details and sample code. Time dependencies in Hidden Markov models can make the generic methods in WinBUGS very ineffective for estimating model parameters, and I therefore only give efficient Gibbs sampling algorithms in appendix A3. All of the models discussed in this chapter can be used in a multivariate setting, typically by simply replacing the distributions for environments and their priors by their multivariate counterpart.

\subsubsection{Characterizing contingents: a marginal visualization approach}

A contingent in the context of dispersal refers to groups of fish with distinct dispersal histories (Secor 1999). A contingent is thus made up of fish with similar dispersal (migration) history or similar habitat use. This leaves the question of how one defines this similarity within these groups. An intuitive way to do this is to define similarity in terms of time that fish spent together (at the same time) in the same environment. Speaking in terms of the Bayesian models discussed here, this quantity can be expressed as the marginal posterior probability that a set $S$ of individuals are found in the same environment at a uniformly chosen time $\tau$, or

$$
p\left(\xi_{S^{\prime}, \tau}=\xi_{S, \tau} \mid \boldsymbol{y}\right)=\int p\left(\xi_{S^{\prime}, \tau}=\xi_{S, \tau} \mid \theta\right) p(\theta \mid \boldsymbol{y}) d \theta
$$

where $\mathrm{S}$ and $\mathrm{S}^{\prime}$ are disjoint sets of fish. This can be estimated from $q=(1 \ldots Q)$ MCMC samples of the posterior distribution for model parameters $\theta$ as

$$
p\left(\xi_{S^{\prime}, t}=\xi_{S, t} \mid \boldsymbol{y}\right)=\frac{1}{Q T} \sum_{q=1}^{Q} \sum_{t=1}^{T} p\left(\xi_{S^{\prime}, t}=\xi_{S, t} \mid \boldsymbol{y}, \theta_{q}\right)
$$

and may be seen as a time averaged form of a co-assignment probability (see Dawson \& Belkhir 2001 for a derivation of this probability). It further marginalizes over (integrates out) unknowns in the model and thus reflects uncertainty in parameter estimates.

This definition of (probabilistic) similarity of the time series has a number of advantages. In fact, this quantity is readily obtained from any MCMC 


\section{Chapter 5. A Bayesian framework for characterizing fish dispersal}

implementation of the above described models: one must simply keep track of the assignment vector $\boldsymbol{\xi}$ at each iteration of the sampler. Furthermore, this definition of similarity does not involve the actual labels of environments (or states) and is therefore invariant to label switching. This is a desirable property since it assures that even without restrictive priors on environment signatures one can still define similarity. Lastly, this probability can be used in an agglomerative algorithm such as the exact linkage algorithm (Dawson \& Belkhir 2009). This algorithm produces a tree based on co-assignment probabilities such as defined above, where the node height is equal to the posterior co-assignment probabilities of the merged sets. Contingents can be readily inferred on such a tree by assign them a marginal probability of belonging to the same group.

I introduce a second marginal posterior distribution over the number of individuals $n_{i, t}$ which at time $t$ are associated with the same environment as fish $i$. Again, this quantity is easily computed from the MCMC sequence used to estimate the model and it too is invariant to label switching. Given that this probability is time indexed, it provides information about temporal structure of the data. Intuitively, this number will coincide for fish that are associated with the same environment at time $t$, and if there is synchronous movement then these numbers will be constant for the members of a contingent. If trajectories diverge, it can be easily seen where contingents split or merged on their dispersal pathways. The two marginal distributions are visualized in a joint plot which associates the tree of coassignment probabilities with a matrix image of the $n_{i, T}$ matrix. Such a representation is particularly useful for multivariate applications in which visual differentiation of groups is not straightforward.

\subsection{Illustration and validation}

I illustrate the merit of the above mentioned models with a series of simulation experiments. The first experiment includes four distinct scenarios of increasing complexity to test the performance of different mixture model setups as well as a 


\subsection{Illustration and validation}

flexible HMM setup for comparison. A second simulation experiment illustrates how this method can be used to visualize migratory contingents from multivariate data, even without specific knowledge of the number of distinct environments which fish may have resided in. I lastly demonstrate how this modelling framework can be used on real data to identify plasticity of age at freshwater emigration of Chinook salmon.

\subsubsection{Simulation Experiment I: Increasingly complex simulation scenarios}

The first scenario simulated a case of two environments or water-masses, with each environment characterized by a single normal distribution. Each of 100 simulated fish, for which I simulated transects of 50 individual scans, spent variable amounts of time in each water-mass. Such a scenario could reflect diadromous fish which spend variable amounts of time in chemically well characterized habitats (i.e. Miller et al. 2010). The second scenario was designed as a more complex dispersal scenario in which fish again spend variable amounts of time in one of two environments, however one environment is characterized by a number $(K=3)$ of distinct chemical patterns in collected recruits (i.e. different tributaries or spawning locations). For the simulation there are three distributions of elevated chemistry and a single environment with lower chemistry (Table 5.1).

The third scenario is similar to the second in that every simulated fish originates in one of $k=3$ locations of elevated chemical concentration, however I introduce an autocorrelation coefficient of 0.5 to simulate the fact that changes in otolith chemistry may not be instantaneous.

The last scenario is a relatively complex extension of the second in that each fish may visit any water-mass on a $3 \times 3$ grid (Figure 5.4) with a probability determined by a transition matrix. Residence in a water-mass is chosen with 'base' probability $p_{k, k}=m$. The probability of moving to an adjacent water-mass $\mathrm{j}$ on the grid is $p_{k, j}=\left(1-p_{k, k}\right) / c$, where $c$ is the number of adjacent cells. To simulate aggregative behaviour these probabilities are then transformed to take into 
account the number of larvae in the adjacent cell: the more larvae in each cell the higher the probability of moving to this cell.

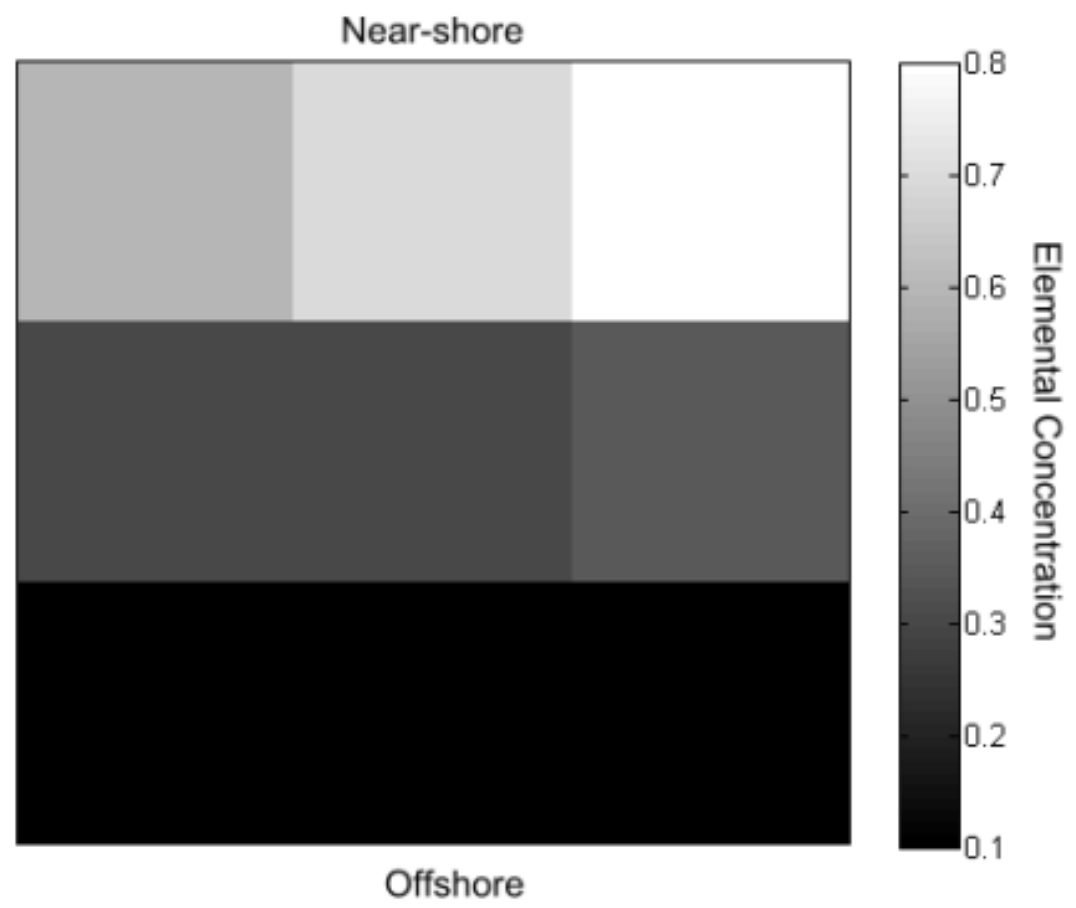

Figure 5.4: The $3 \times 3$ grid of (hypothetical and arbitrary) near-shore to offshore variations in elemental concentration used for simulation scenario 4 of experiment 1.

Parameters for all scenarios are given in Table 5.1. For each scenario I tested a set of models. The simplest of these included a mixture model of two water-masses with a common mixing proportion ( $\mathrm{MM})$ and a random effects mixture model with a common mixing proportion (the prefix RFX- denotes a random effects formulation for the water-mass mean). I repeated the analyses with the same models but with individual $\pi$ (suffix -1 ) as well as with a DP random effects mixture model. Two HMM formulations, one with a single transition matrix for all fish and another with individual transition matrices for each fish were 


\subsection{Illustration and validation}

implemented for comparison. Of particular interest is the parameter $\pi$ in the mixture models, as well as partitions over water-mass assignments (see below).

Table 5.1: Simulation parameters for experiments I (scenarios 1-4) and II (scenario MV): $\mu_{o}$ and $\mu_{n}$ are means of two distinct water-masses (i.e. offshore and near-shore or fresh- and ocean water). $\sigma$ denotes the standard deviation, except for the multivariate scenario (MV) for which it is the diagonal of the covariance matrix. A is a first order autoregressive parameter.

\begin{tabular}{|c|c|c|c|c|c|}
\hline Experiment & Scenario & $\mu_{n}$ & $\mu_{o}$ & $\sigma$ & A \\
\hline \multirow{4}{*}{ I } & 1 & 0.75 & 0.25 & 0.15 & 0 \\
\hline & 2 & $0.45,0.75,0.95$ & 0.25 & 0.1 & 0 \\
\hline & 3 & $0.45,0.75,0.95$ & 0.25 & 0.05 & $1 / 2$ \\
\hline & 4 & $0.6,0.7,0.8$ & $0.1,0.3,0.35$ & 0.05 & 0 \\
\hline \multirow{4}{*}{ II } & \multirow{4}{*}{ MV } & \multirow{4}{*}{$\begin{array}{l}{\left[\begin{array}{llll}0.05 & 0.55 & 1.05 & 1.05\end{array}\right],} \\
{\left[\begin{array}{llll}0.55 & 0.05 & 0.55 & 0.55\end{array}\right],} \\
{\left[\begin{array}{llll}1.05 & 1.05 & 0.55 & 0.55\end{array}\right]}\end{array}$} & {$[0.14$} & {$[0.14$} & \multirow{4}{*}{0} \\
\hline & & & & & \\
\hline & & & 0.09, & 0.22 & \\
\hline & & & $0.28]$ & $0.14]$ & \\
\hline
\end{tabular}

\subsubsection{Simulation Experiment II: Contingents from multivariate data}

This experiment serves to demonstrate how the proposed modelling approach in conjunction with the marginal visualization methods can be used to find contingents. The proposed visualization technique is particularly useful in a multivariate setting (four variables - Table 5.1) where simple plots of chemical time-series of multiple elements can be hard to interpret visually. I used 20 randomly selected fish from a multivariate version of scenario 2 in experiment I to illustrate the approach. I deliberately chose difficult parameter values (i.e. relatively high variances, some overlap in distributions) for this simulation. In this 


\section{Chapter 5. A Bayesian framework for characterizing fish dispersal}

setup it would be difficult to estimate the true movement patterns from one element alone. I used the non-parametric DP-RFX-MM model to resolve similarities in dispersal histories and examine how well the non-parametric method performs at estimating common dispersal patterns and the number of water-masses present in the profiles. Both may not be immediately obvious from multivariate time series.

\subsubsection{Assessing models: posterior predictive checks and model selection}

I applied the above mentioned models to the simulation scenarios to find the limits and strengths of the respective models. To compare models in terms of their adequacy for the data at hand I employed a number of model selection tools.

The natural approach for this in a Bayesian analysis is the Bayes factor, which is the ratio of integrated (also called marginal) likelihoods, in other words the ratio of model likelihoods integrated over unknown parameters (Kass \& Raftery 1995). The Bayes factor can be thought of as a Bayesian analogue of the likelihood ratio test, where rather than maximizing the likelihood with respect to unknown parameters, these are integrated over in the posterior distribution. The advantage of the Bayes factor is that it automatically penalizes complex models if these are not justified. A ratio of 10:1 is generally seen as strong evidence in favour of the better model, a ratio of >100:1 shows decisive evidence in favour for the winning hypothesis (Kass \& Raftery 1995). The Bayes factor is somewhat difficult to compute, especially in more complex models such as DP models, and I reserved this for models of real data. Specifically, I used the method of Chib (1995) to estimate the marginal likelihood in the next section (5.3.4). The use of informative priors in that section assures that sensitivities of Bayes factors to non-informative priors are of minor concern in this case.

For the simulation studies, I examined estimated quantities and looked at data generated from the posterior predictive distribution to check for consistency of these mixture models with the real data - if simulated data from this distribution is far from the real data in terms of predefined characteristics (i.e. the spread in the data), the model is deemed inadequate (Gelman et al. 2003). While this does not 


\subsection{Illustration and validation}

give a measurable quantity for model comparison in a Bayesian sense, it will indicate model adequacy and can thereby serve to point out (in-) appropriate models.

To evaluate model fit I checked whether the proposed models were able to reflect the spread in the data by calculating the posterior predictive test quantity

$$
E(\Delta Y)=\frac{1}{Q} \sum_{q=1}^{Q}\left(\max \left(\boldsymbol{y}_{\boldsymbol{i}}\right)-\min \left(\boldsymbol{y}_{\boldsymbol{i}}\right)\right)-\left(\max \left(\boldsymbol{y}_{\boldsymbol{i}, \boldsymbol{q}}^{\boldsymbol{M}}\right)-\min \left(\boldsymbol{y}_{\boldsymbol{i}, \boldsymbol{q}}^{\boldsymbol{M}}\right)\right)
$$

where $\boldsymbol{y}_{\boldsymbol{i}, \boldsymbol{q}}^{\boldsymbol{M}}$ is data simulated from the posterior predictive distribution $p\left(\boldsymbol{y}^{\boldsymbol{M}} \mid \boldsymbol{y}\right)=\int p\left(\boldsymbol{y}^{\boldsymbol{M}} \mid \theta\right) p(\theta \mid \boldsymbol{y}) d \theta$ for concentrations $\boldsymbol{y}$ and the expectation $E$ is calculated over $Q$ MCMC samples from the posterior distribution. For mixture models I also calculated $E(\Delta \pi)=\frac{1}{N Q} \sum_{q=1}^{Q} \sum_{i=1}^{N}\left(\pi_{i, q}-\pi_{i, q}^{M}\right)$ for all simulations, where $\pi^{M}$ is the estimated posterior distribution of the paramter $\pi$ in the model. The latter quantity simply verifies whether the model accurately estimates the true mixing proportions.

To illustrate the advantages and drawbacks of these models, I compared the modal partitions of each model to the original partition (i.e. the mode of the posterior distribution of $\boldsymbol{\xi}$ ). A partition is simply the set of assignments of scans to water-masses (the $\xi s$ in the model). In the case of two water-masses, this would be a set of 0 s and 1 s for each fish. With DP random effects models, one could also choose to use the partition that includes assignment to a given random effects water-mass. One would then use a set of arbitrary numbers for each water-mass. The same applies to setups with more than two water-masses. The mean partition is then simply the partition that minimizes the sum of squared distances from the partitions observed during the MCMC. These partitions however are not invariant to label switching and I use them for illustrative purposes only - all models used for simulations use identifiability constraints on the means of water-masses. 


\section{Chapter 5. A Bayesian framework for characterizing fish dispersal}

\subsubsection{Application to Chinook salmon}

To test the performance of these models in the real world, I applied a hidden Markov model to a set of Barium (Ba) and Strontium (Sr) profiles across otoliths of adult Chinook salmon. These data have previously been analysed by Miller et al. (2010) to estimate fork length at freshwater emigration (FL) of fish originating in the Central California Valley. Their study found that fish emigrate at a variety of FLs, and that medium sized individuals ('parr') represent the majority of emigrating juveniles.

FL at emigration was calculated from otolith width (OW) at emigration, with OW itself taken at a threshold point where substantial changes in otolith chemistry occur. Specifically, at freshwater emigration, Sr tends to increase, reflecting higher availability with increasing salinities. Similarly, Barium tends to decrease substantially as salinity increases. A series of experiments and empirical observations lead to predictions about otolith chemistry in the different environments of interest. These predictions in turn allowed the authors to define a threshold for ocean and freshwater signatures. A third category, brackish water, seemed to be present in the measured chemistry, with higher Barium than ocean waters and Strontium values approaching those of ocean water.

The proposed thresholds depended on a number of models and observations relating otolith chemistry to water chemistry. As noted by Miller et al. (2010), the observed chemistry of some of the adult fish collected falls outside of the predicted confidence intervals. For ocean residence for instance, only $44 \%$ of fish fell within the predicted Sr range, while only $12 \%$ fell within the predicted Ba range. There is thus clearly a limit to the underlying models and hence the proposed thresholds. I show how HMMs can be used to estimate habitat transitions from both the previous predictions and the current data at hand. I show how the methods presented in this chapter can be used to obtain (marginal) posterior over parameters of interest in the original study, as well as additional quantities estimated from the models. 


\subsection{Illustration and validation}

I used a hidden Markov model to reconstruct movements since these tend to filter more noise and will allow for a more accurate estimation of the OW at emigration. Random effects were used for the freshwater signatures, reflecting the contribution of different tributaries and/or varying strength in maternal/physiological effects on observed freshwater signatures. I added a third category of brackish water and tested this model against a model with only two water-masses using the Bayes factor. I could have fixed the HMM transition matrix since we know that across the profile, which spans the entire otolith, one transition from ocean- to freshwater and one transition from fresh- to ocean water will be observed. Given the relatively clear pattern however, results are virtually identical and I report results with estimated transition matrices.

While it is possible to include the regression models that lead to predictions about otolith chemistry explicitly in the Bayesian framework - the prior itself could issue from an underlying regression relationship including previous data - I restrict the analysis here to the core part of the proposed models. I therefore use the predictions as they appear in the original paper and use them to define priors over water-mass parameters. Similarly, I adopt regression coefficients and prediction errors from the original study and use Monte Carlo integration to find the marginal (pseudo) posterior distribution of FL. Note however that the possibility of integrating multiple data types for a combined model in this fashion is another powerful argument for a Bayesian analysis. As priors for water masses I used a conjugate Normal-Inverse- $\mathrm{X}^{2}$ distribution:

$$
\begin{aligned}
\mu & \sim N\left(\mu_{0}, \frac{\sigma_{0}}{\kappa_{0}}\right) \\
\sigma^{2} & \sim \operatorname{Inv} \chi^{2}\left(\nu_{0}, \sigma_{0}\right)
\end{aligned}
$$

This distribution has four parameters: the prior mean $\left(\mu_{0}\right)$ and variance $\left(\sigma_{0}\right)$, as well as two parameters that encode uncertainty about the prior: The prior $\nu_{0}$ corresponds to the number of prior accounts of the variance (i.e. how often has this variance been observed in previous studies) while $\kappa_{0}$ indicates how sure we are of the prior mean. Furthermore, the prior of the mean depends marginally on that 


\section{Chapter 5. A Bayesian framework for characterizing fish dispersal}

for sigma: the smaller the variance of a water-mass, the more certainty we should have about its actual mean. The first two parameters can be obtained from the original study, while the latter two have to be set with respect to the data at hand, which contains some $N * T \sim 50000$ individual scans from 47 fish. Since we only have one prior study for oceanic water-masses, I set this prior to be somewhat vague as opposed to that for freshwater, where we have 22 accounts for $\mathrm{Ba}$ predictions and 27 predictions for Sr distributions in individual contributing rivers. The prior for brackish water is a vague guess and I do not wish to put much weight on it. For the random effects, the prior accounts $\nu_{0}$ reflect the number of prior studies reported in Miller et al. (2010). It is relative to the number of random effects, which is the same as the number of fish (47). Table 5.2 gives the parameter values used in the present study.

Table 5.2: Prior parameters used for the Chinook salmon dataset. $\mu_{0}$ is the prior mean, $\sigma_{0}$ the prior standard deviation, $\kappa_{0}$ parameterizes the uncertainty around the prior mean, $\nu_{0}$ that for the prior variance (prior degrees of freedom). $\kappa_{0}$ and $\nu_{0}$ were set with respect to the total number of observations $N * T$. Sr is measured in $\mathrm{mmol}^{\mathrm{mol}}{ }^{-1}$, Ba figures are in $\mu \mathrm{mol} . \mathrm{mol}^{-1}$.

\begin{tabular}{|c|c|c|c|c|}
\hline Water-mass & $\mu_{0}[S r \mid B a]$ & $\sigma_{0}[S r \mid B a]$ & $\kappa_{0}[S r \mid B a]$ & $\nu_{0}[S r \mid B a]$ \\
\hline Coastal Ocean & $1.98 \mid 1$ & $0.045 \mid 0.14$ & $N * T / 20$ & $N * T / 10$ \\
\hline Brackish & $1.68 \mid 3.41$ & $0.045 \mid 1.41$ & $N * T / 500$ & $N * T / 5000$ \\
\hline Fresh (RFX) & $1.00 \mid 6.93$ & $0.090 \mid 3.40$ & $27 \mid 22$ & $27 \mid 22$ \\
\hline Fresh (Variance) & - & $0.35 \mid 1.55$ & $N * T / 5$ & $N * T / 5$ \\
\hline
\end{tabular}

\subsection{Results}

\subsubsection{Simulation experiment I}

Mixture models without individual mixing proportions $\pi$ generally performed less well than models that included individual effects (Table 5.3). This is due to the fact that the common parameter $\pi$ amongst fish penalizes differences in movement patterns between fish or groups of fish. This in turn affects estimates of other model parameters and as a result the posterior predictive distribution cannot 


\subsection{Results}

reflect the simulated data. The relatively good performance of these two models for scenario 4 is due to the low variance used for this simulation. Indeed, this simulation experiment is a compromise between adding realism and maintaining some necessary identifiability of water-masses.

The random effects formulation of this model (RFX-MM) further illustrates the interplay between $\pi$ and the identifiability of water-masses (i.e. their variance relative to the difference in means). The model performs at par with individual effects models for simulations 2 and 4, while performing markedly worse for the remaining two scenarios (Table 5.3). In scenario 2 and 4 the model is flexible enough to fit water-mass distributions that closely match those of the actual simulation. Given that the variance is lower than in scenario 1 , the added identifiability outweighs the common mixture parameter and leads to relatively good results. This identifiability is weakened in scenario 3 by the autoregressive parameter, which is reflected by a poor fit of the posterior predictive distribution to the original data. Within models with individual effects for $\pi$, those models that included random effects for water-masses outperformed the simple mixture in all but the simplest simulation (Scenario 1). Results from both random effects formulations were comparably good. Hierarchical formulations for $\pi$ produced similar results to individual effects, though generally performed slightly worse due to parameter estimates being shrunk to the underlying random effects distribution.

Random effects HMMs performed very well for simple scenarios (1 \& 4) but did not do as well as random effects mixture models for more complex scenarios. The mean partitions give some clues as to why this is so (Figure 5.5). The filtering effect is clearly visible in the mean partitions for scenarios 1 and 3 . In both cases the partition obtained from the mixture models reveals that some of the noise is attributed to a transition between water-masses. Especially the mixture model with single mixing parameter $\pi$ attributes much of the noise to movement patterns. 
Table 5.3: Simulation results for each scenario summarized the posterior predictive test quantity $E(\Delta Y)$ as well as by $E(\Delta \pi)$ and the corresponding standard deviation $s d(\Delta \pi)$. $\mathrm{E}$ denotes the expectation in both cases. For both test quantities, lower absolute values for discrepancies between model posterior distributions and simulated data indicate better model fit. Model abbreviations are $\mathrm{MM}=$ mixture model, $\mathrm{HMM}=$ hidden Markov model, prefix RFX $=$ random effects for water-masses, prefix DP $=$ Dirichlet Process, suffix $-1=$ individual $\pi$.

\begin{tabular}{|c|c|c|c|c|c|c|c|}
\hline & MM & $\begin{array}{l}\text { RFX- } \\
\text { MM }\end{array}$ & MM-I & $\begin{array}{l}\text { RFX- } \\
\text { MM-I }\end{array}$ & $\begin{array}{l}\text { DP-RFX- } \\
\text { MM-I }\end{array}$ & $\begin{array}{l}\text { RFX- } \\
\text { HMM }\end{array}$ & $\begin{array}{l}\text { RFX- } \\
\text { HMM-I }\end{array}$ \\
\hline Scenario & \multicolumn{7}{|c|}{$E(\Delta Y)$} \\
\hline 1 & 0.32 & -0.21 & 0.027 & -0.038 & 0.022 & 0.022 & 0.020 \\
\hline 2 & -0.17 & -0.030 & -0.14 & -0.029 & -0.030 & -0.10 & -0.067 \\
\hline 3 & -0.28 & -0.42 & -0.22 & -0.065 & 0.027 & -0.19 & -0.14 \\
\hline \multirow[t]{2}{*}{4} & -0.090 & -0.047 & -0.13 & -0.020 & -0.041 & -0.035 & -0.024 \\
\hline & \multicolumn{7}{|c|}{$E(\Delta \pi)[s d(\Delta \pi)]$} \\
\hline 1 & - & - & $\begin{array}{l}-0.0018 \\
{[0.068]}\end{array}$ & $\begin{array}{c}-0.0025 \\
{[0.068]}\end{array}$ & $\begin{array}{c}-0.0019 \\
{[0.068]}\end{array}$ & - & - \\
\hline 2 & - & - & $\begin{array}{l}-0.17 \\
{[0.28]}\end{array}$ & $\begin{array}{c}-0.0049 \\
{[0.086]}\end{array}$ & $\begin{array}{c}0.00042 \\
{[0.076]}\end{array}$ & - & - \\
\hline 3 & - & - & $\begin{array}{l}-0.060 \\
{[0.47]}\end{array}$ & $\begin{array}{l}-0.040 \\
{[0.084]}\end{array}$ & $\begin{array}{c}-0.0033 \\
{[0.088]}\end{array}$ & - & - \\
\hline 4 & - & - & $\begin{array}{c}-0.24 \\
{[0.21]}\end{array}$ & $\begin{array}{l}-0.034 \\
{[0.086]}\end{array}$ & $\begin{array}{c}-0.0026 \\
{[0.068]}\end{array}$ & - & - \\
\hline
\end{tabular}

The HMMs clearly give a better approximation of the true partition for scenario 1: The noisy observations rarely contribute to changes in the (filtered) sequence of environments (Figure 5.5). Scenario 3 however demonstrates a drawback of this filtering in difficult situations. When the true parameters are hard to estimate, the estimated partition may be substantially different form the true partition. Strong constraints on the transition probabilities (see considerations 


\subsection{Results}

above) are then paired with only vague ideas about parameters of water-masses. This results in excessive smoothing and a poor estimate of the actual movement pattern (Figure 5.6).

\subsubsection{Simulation experiment II}

The multivariate experiment mainly serves illustrative purposes. The model does very well at estimating the true number of contributing distributions for the random effect (Figure 5.7). There is little support for more than three contributing sources, and no support for less than three source environments.
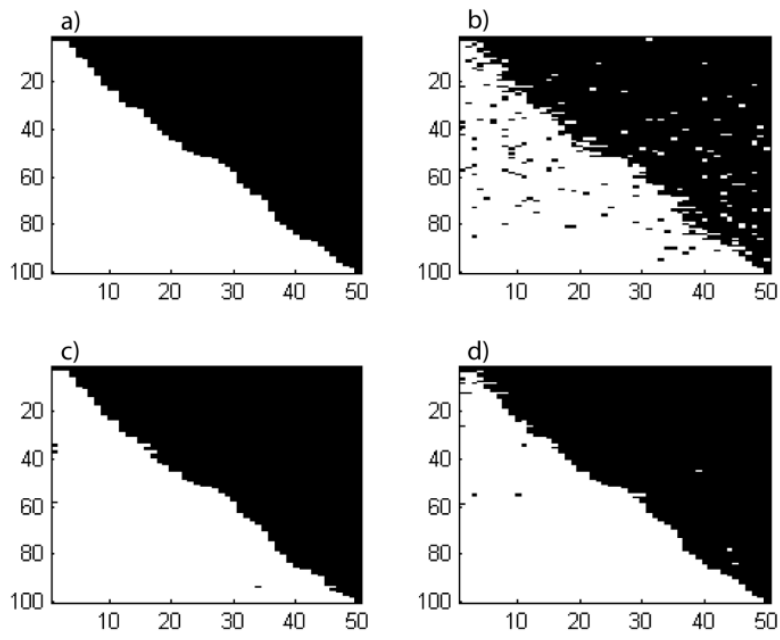

Figure 5.5: (a) True and (b-d) estimated partitions for a (b) random effects mixture model, (c) a hidden Markov model (HMM) with a common transition matrix and (d) a HMM with individual matrices for each individual fish. Each line is the partition of the simulated profile for a single fish into one of two environments (white vs. black), while each column is a separate simulated scan along the otolith growth axis. 

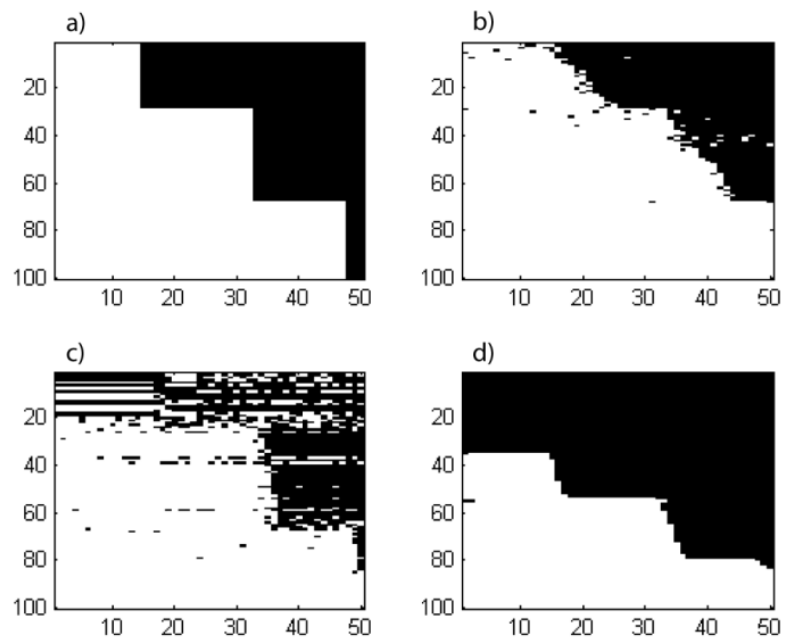

Figure 5.6: (a) True and (b-d) estimated partitions for (b) a Dirichlet process random effects mixture model, (c) a mixture model with a single mixture proportion over all fish and (d) a hidden Markov model with a single transition matrix. Each line is the partition of the simulated profile for a single fish into one of two environments (white vs. black), while each column is a separate simulated scan along the otolith growth axis.

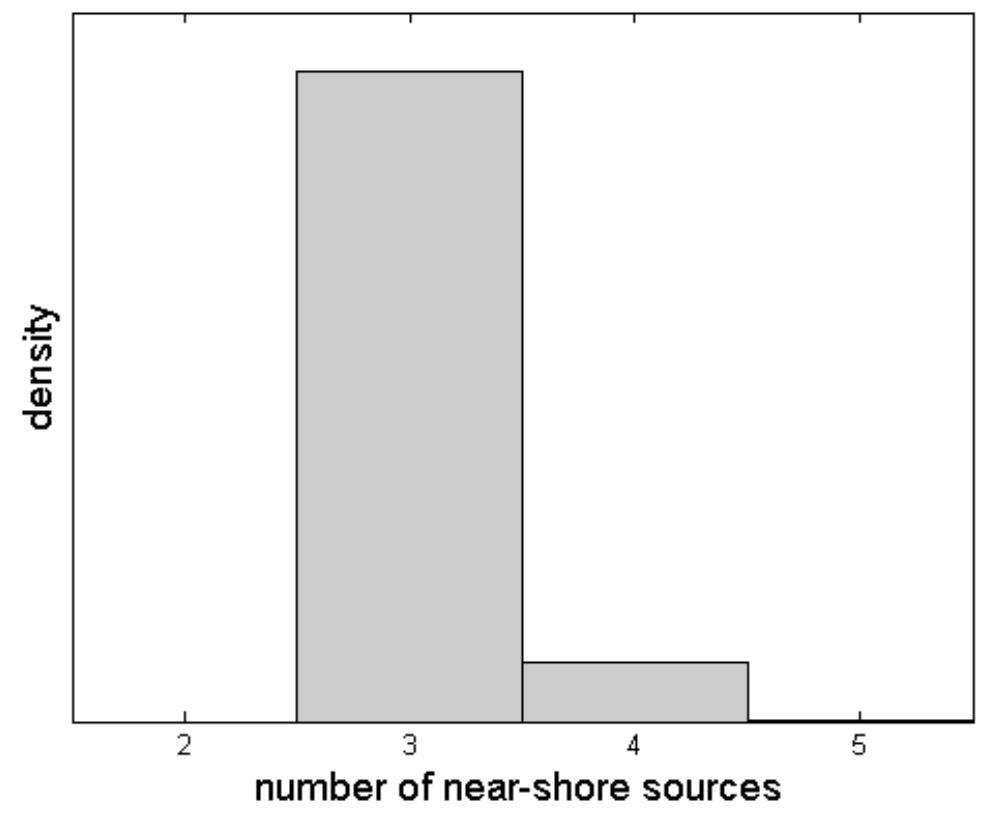

Figure 5.7: Marginal posterior distribution over the number of contributing near-shore sources in simulation experiment II. 


\subsection{Results}

The visualization method clearly identifies four migratory contingents (Figure 5.8). From the time resolved plot it is easily seen that three of these contingents originate from different source environments and spend most of their time in these environments. The remaining contingent is made up of fish which originate in three different environments, but spend most of their time together offshore. The coassignment probabilities between contingents are very low. This reflects uncertainty in water-masses as well as the fact that with this definition of similarity, co-assignment is calculated between all individuals in a set. The time resolved plot clearly shows that for one of the water-masses there is considerable uncertainty with regards to the nature of this water-mass. Other water-masses are easily identified from the time-resolved plot.

\subsubsection{Application to Chinook salmon}

The model with three water-masses performed decisively better than the two water-mass model, the Bayes factor estimate in favour of the former being 33816 . To check for the influence of the priors I examined the posterior distributions of water-mass parameters (Figure 5.9). The figure clearly shows that some of the priors missed the mark. Ocean Ba stands out as it defies both the prior assumptions as well as findings reported in the original study, which finds that ocean Barium is substantially lower than predicted. The results from the HMM however showed higher than expected $\mathrm{Ba}$ (Figure 5.9). From Figure 1 it is evident that $\mathrm{Sr}$ is substantially more informative than $\mathrm{Ba}$, and the model is thus largely driven by changes in Sr. Since the relative amount of $\mathrm{Sr}$ incorporated into the otolith decreases with respect to increasing salinity, brackish and ocean waters are difficult to distinguish from $\mathrm{Sr}$ alone, and the model sometimes assumed ocean residence when $\mathrm{Sr}$ approaches the $\mathrm{Sr}$ ocean mean. Since $\mathrm{Sr}$ is more informative overall, observations were at times assigned to ocean residence when fish were still likely in estuarine waters with higher $\mathrm{Ba}$, thereby driving up the ocean $\mathrm{Ba}$ mean. 


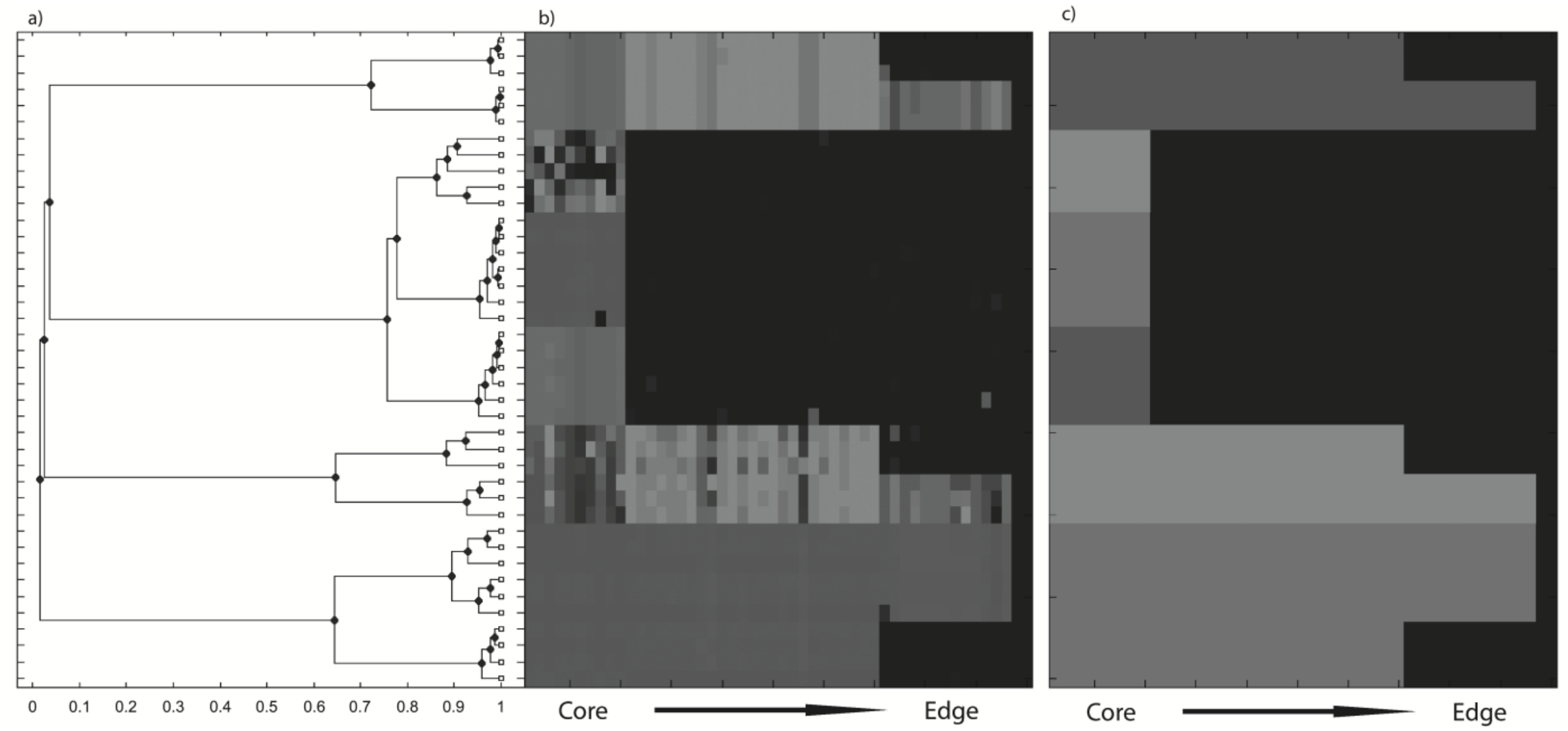

Figure 5.8: Visualization of marginal similarity of transects from simulation experiment II. a) The tree displays average marginal similarity: Co-assignment probabilities (node heights in the tree) are marginal over the i) estimated number of water-masses and ii) parameter uncertainty. b) The time resolved plot shows the marginal number of fish in the same water mass as fish $i$ at time $t$, identical shades of grey thus indicate common dispersal paths for a given group identified from the tree. c) The true partition including the three different contributing random effects sources, visible as like shades of grey. 


\subsection{Results}

The random effects mean for $\mathrm{Ba}$ was substantially higher than previous data would suggest. This was driven by some fish with very high $\mathrm{Ba}$, as reported in the original study. Similarly, brackish water Sr was lower than ocean $\mathrm{Sr}$ values and lower than the vague prior guess suggested (Figure 5.9). Again, this occurred due to brackish water signatures with high Sr being classified as ocean residency; thereby driving down the brackish water mean Sr. Furthermore, the natal region of the otolith of some fish displayed very strong maternal effects in otolith chemistry, especially for $\mathrm{Sr}$ which is generally high in this region. These observations were often assigned to brackish water residence, thereby obscuring the true pattern. These shifts in beliefs are not very concerning however, since the main focus is to estimate $F L$ at emigration from freshwater, the driving parameters of which are in line with prior expectations and the observed shift in $\mathrm{Ba}$ is in a direction which is of no immediate concern.
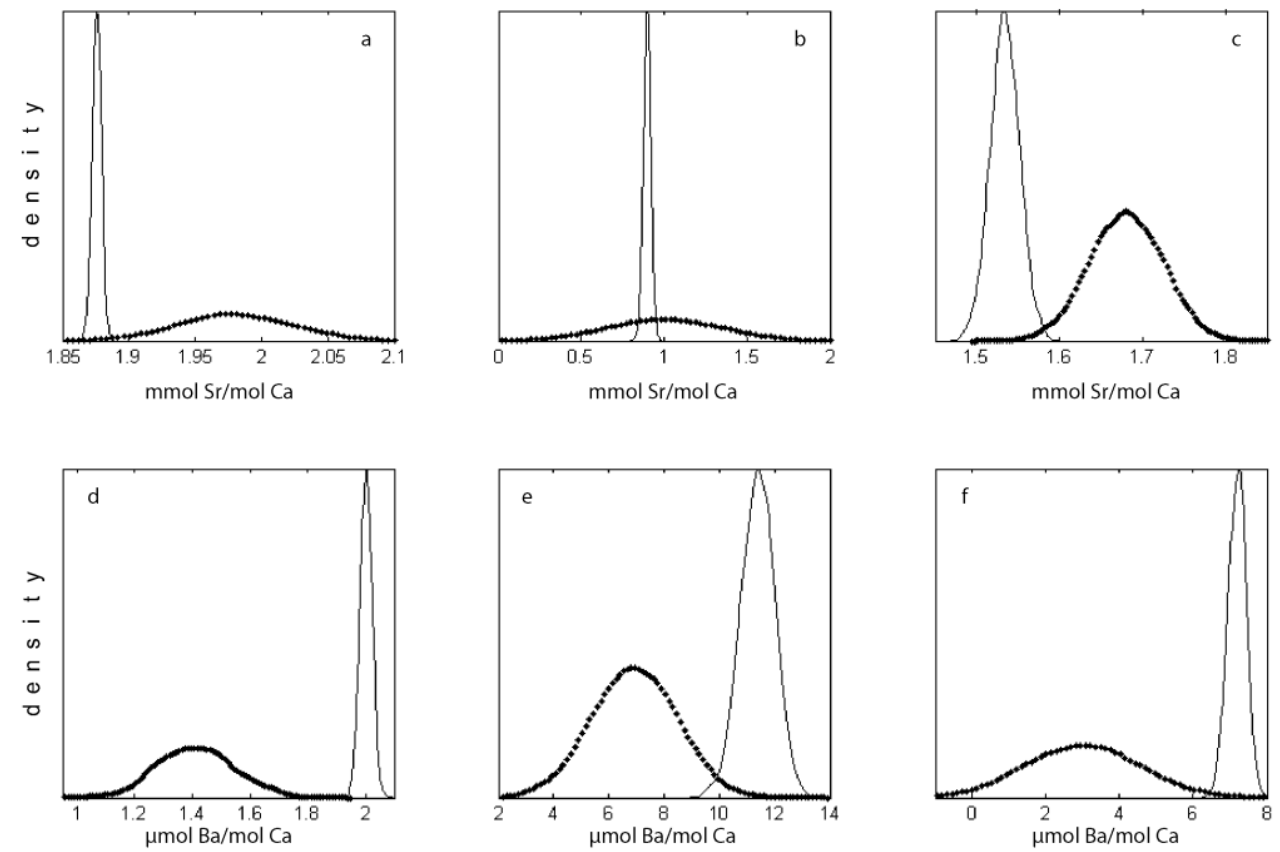

Figure 5.9: Prior (dotted) versus posterior (line) distributions of water-mass means of Strontium ( $\mathrm{Sr}-\mathrm{a}-\mathrm{c}$ ) and Barium (d-f) for the three component model for Chinook salmon, for ocean- $(a, d)$, fresh- $(b, e)$ and brackish water $(c, f)$. 
Miller et al. (2010) found that medium sized individuals (parr) constitute the dominating contingent $(47.5 \pm 0.8 \%)$, followed by large individuals (smolt: $32.4 \pm 6.2 \%$ ) and small individuals (fry: $20.1 \pm 5.4 \%$ ). The marginal posterior distributions of emigrating contingents (Figure 5.10a) revealed a slight upwards shift in the estimated $\mathrm{FL}$ at emigration from the HMM: smolt (with a posterior mean of $34.83 \%, 95 \%$ credible interval [19.15\%;56.06\%]) make up a slightly larger proportion of the emigrating juveniles than reported in the original study. According to model results, parr (46.55\%, [36.17\%;55.32\%]) and fry (18.65\%, [10.64\%;34.04\%]) account for slightly fewer emigrants than originally reported, but were well within the confidence envelopes of the original study. The credible intervals were asymmetric and considerably larger than the originally reported confidence intervals, owing to uncertainty about the $\mathrm{OW}$ at emigration. Comparing the distribution of juvenile sizes at freshwater emigration (Figure 5.10b) shows two dominating peaks in sizes of emigrating juveniles, which are made up of fry and parr for one and parr and smolt for the second peak. A third and minor peak can be found at larger sizes. Small peaks at very small sizes $(<45 \mathrm{~mm})$ are likely a modelling artefact of freshwater scans on one side of the otolith occasionally being attributed to brackish water residence. 


\subsection{Discussion}
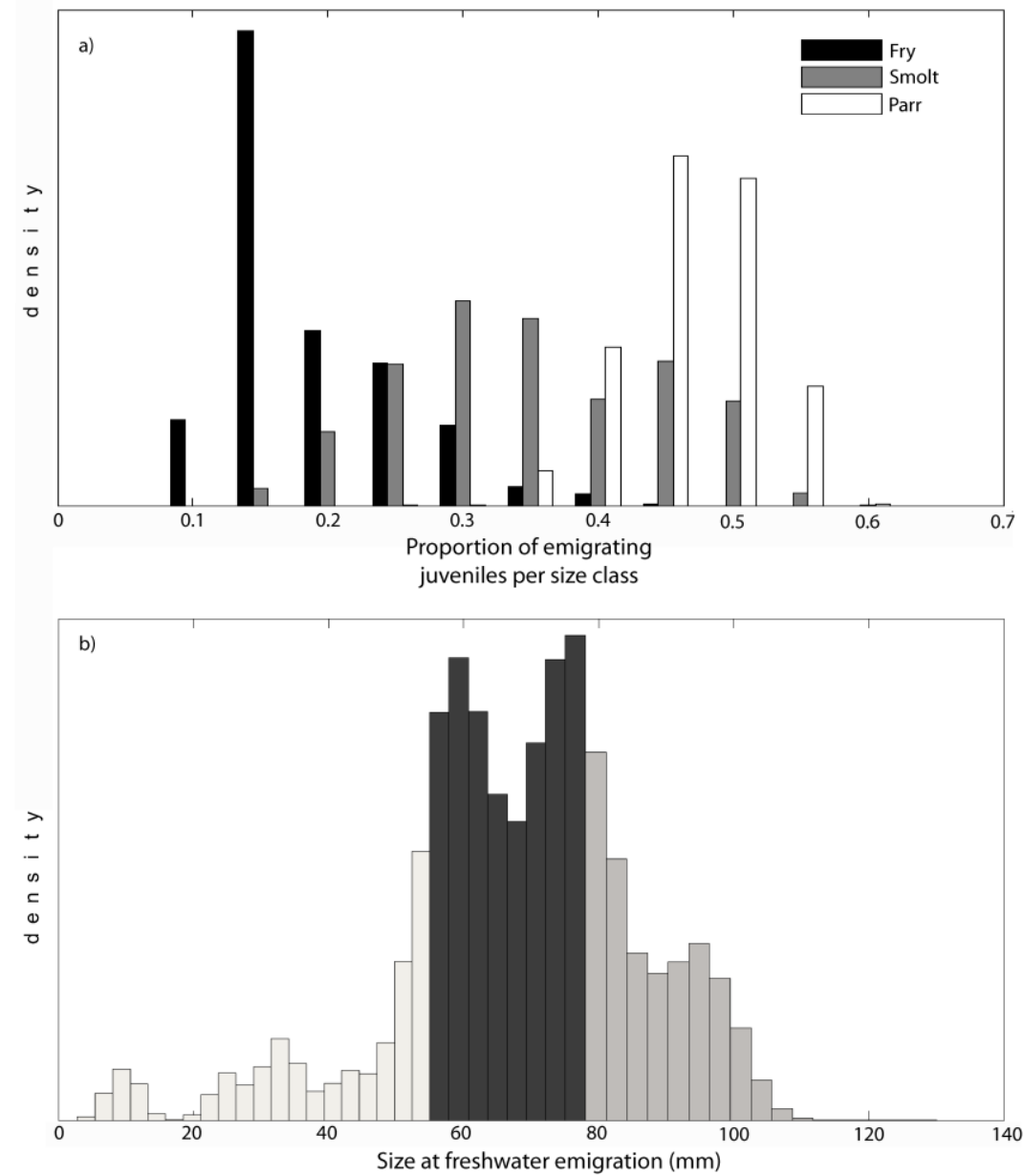

Figure 5.10: Histograms of a) the marginal posterior distribution over per cent emigrating size classes and b) posterior distribution of size of Central California Valley Chinook salmon at freshwater emigration, colours define size classes as labelled in Miller et al. (2010).

\subsection{Discussion}

The modelling approach presented here provides a general framework for the analysis of otolith chemical profiles, and addresses some fundamental issues and questions often asked from this sort of data, such as the identification of migratory contingents from models of otolith transects. Generative models depend on hypotheses about the data 
generating mechanism. In the case of chemical transects across otoliths, one needs to specify hypotheses about water-masses that could be reflected within these transects. Often, strong prior information is available about patterns (such as for $\mathrm{Ba}$ and $\mathrm{Sr}$ ) which we can exploit (i.e. Elsdon \& Gillanders 2003, Fablet et al. 2007). In other cases however, the observed patterns cannot be a priori tied to a known number of environments (i.e. Shima \& Swearer 2009a). In this case the present framework can be used to compare competing models about different potential hypotheses about dispersal or infer the number of distinct water masses directly using the DP extension. Thus, while specifying a specific generative mechanism may be difficult at times, these models can still provide insight about a likely generating mechanism. I also found that it helps to plot the global frequency distribution of the data along with the actual transects when thinking about such hypotheses (i.e. Figure 5.1). The underlying hypotheses of these models are verifiable, and beliefs about models and their parameters can be updated in the light of further studies which test underling hypotheses. Furthermore, credible intervals for each model parameter provide us with an idea of how much the inferences from each model are worth. For models that produced poor results in the simulations, I could generally observe that some parameters, such as random effects for instance, had absurdly large credible intervals.

In order to construct generative models and specify their distributions over water-masses, the meaning of water-masses or environments needs to be clearly defined. Most importantly, these need to be identifiable from the data. Having an idea of the scales over which (otolith-, and ideally water-) chemistries change is thus indispensible (Elsdon et al. 2008). Prior assumptions about such scales through prior distributions on water-mass variances can be a way to explicitly define the scale on which to consider a water-mass. In the Chinook example I place an informative prior on the variance of the water-masses, which are thereby defined to be 'within 


\subsection{Discussion}

river' for freshwater signatures for example. While the Dirichlet Process models aim to estimate the number of water-masses in a given dataset, the same consideration applies: we can only identify the number of distinct environments in the model if these are identifiable in the first place, or if we know the scale of variation of individual water-mass signatures.

The application to Chinook salmon illustrates the merit and validity of the Bayesian approach, but also some possible pitfalls. The posterior distributions for emigration percentages for the three size classes provide a way to assess uncertainty in estimates of freshwater residence: distributions over the percentage of emigrating fry and parr for instance are highly skewed and the posterior mode may provide a better estimate of the true percentage per size class than does the mean. Miller et al. (2010) used thresholds to visually define the transition between habitats. While transitions are relatively clear for most fish, they are rather smooth for some. The definition of this threshold thus clearly presents a degree of subjectivity, which introduces uncertainty in the actual otolith width at emigration. The Bayesian model integrates over this uncertainty, which is evident in considerably larger credible intervals.

One needs to be cautious however in directly interpreting water-mass distributions: the analysis shows that transitional periods and maternal effects can influence these estimates. It is thus likely that any smooth transitions in salinity for instance will somewhat effect other parameters of interest. This may be in part the reason why I observe a slight shift in FL at emigration, and hence in relative percentages of emigrating juveniles for the tree size classes. My estimates are very close to the original estimates however and represent a shift of only one individual per category. Furthermore, simulation scenario 4 indicates that most realistic models perform well even in the presence of transitional zones. 
One of the most important applications of the methods presented here is the identification of migratory contingents. The definition of a contingent is dependent on the number of environments considered as well as the parameters of interest in a particular study. Secor (1999) for instance defines a contingent as "... a level of fish aggregation based upon divergent migratory behaviours or habitat use within a population,...". In the Chinook salmon example these contingents would be fish that spend comparable time in their natal freshwater habitat, but alternative contingents could be fish that spend comparable time in fresh- and brackish water. Using this objective method produces two dominant peaks in the distribution over size at emigration, which may be two main emigrating contingents, each containing individuals from two of the pre-defined size classes (fry \& parr or parr \& smolt respectively). The histogram in (Figure 6a) in Miller et al. (2010) shows a shape which resembles a normal distribution, but this difference may be due to the larger bin size for the histogram in the original study.

The marginal descriptions of the model outputs illustrate the advantage of a fully Bayesian approach: it opens the possibility to define contingents and similarity of dispersal syndromes which integrate over unknown parameters and thereby provide robust descriptions of fish migrations. This approach assumes that the belief that two fish shared a similar dispersal history is lower the more uncertain we are about the number and nature of different water-masses, and hence about a fishes migration history. Uncertainty about water-masses is only present in DP-random effects models, but effects our beliefs in co-assignment probabilities significantly. This definition of similarity makes intuitive sense: the more certain we are about the data generating mechanism and the underlying parameters, the more sure we can be about a fish's dispersal history. Note that this approach is rather different from what researchers often use for this sort of data: A repeated measure ANOVA, for instance, will test for difference 


\subsection{Discussion}

between transects, and migration histories are regarded as not-separable when no significant difference is found. The approach presented here aims to infer similarity: dissimilarity is thus the 'default' and is higher the more uncertainty is represented in the posterior distribution.

One drawback of this marginal method for finding contingents is that it relies on fish profiles being aligned i.e. scans should correspond to the same age or be aligned to correspond. This is the case when profiles run from the otolith core, often identifiable by a distinct peak in Manganese and other elements (Brophy et al. 2004, Ruttenberg et al. 2005), to the edge. For profiles of different lengths, the marginal $n_{i, t}$ (the number of fish in the same environment as fish $\mathrm{i}$ at time $\mathrm{t}$ ) can be adjusted to account for the number of fish still aligned at time $t$. For profiles spanning the entire otoliths such as in the salmon example, an alignment based on a peak in $\mathrm{Mn}$ for instance can still be made if the location of that peak has been identified. Alignment is difficult otherwise, and one has to resort to multiple alignment algorithms.

The Bayesian approach proposed in this study opens the door to more complex analyses. It allows not only for added flexibility, but also for simultaneous estimation of all relevant parameters, which ensures that uncertainty in parameters is handled appropriately. For example, I already mentioned above that it would be possible to integrate other data sources which may specify relationships between water chemistry and otolith chemistry, and allow for the reconstruction of specific dispersal pathways (Royer et al. 2005). The inclusion of prior beliefs about water-mass characteristics provides a way to incorporate knowledge of patterns in otolith chemistry. The model for the Chinook data demonstrates how shifts in belief about such parameters can happen even when drawing on a body of prior studies to define prior distributions. 
An automated approach can provide advantages such as standardization amongst different studies and the elimination of subjective thresholds and decisions (Fablet et al. 2007, Hedger et al. 2008). The framework proposed here however goes beyond a simple analysis of habitat sequences. The extensions I propose are but some simple additions to these models. Recent advances in movement ecology highlight the potential of latent state models (Patterson et al. 2008, Schick et al. 2008, Patterson et al. 2009). In particular, generative hierarchical models can contribute significantly to mechanistic understandings of animal movement (Schick et al. 2008).

Larval dispersal in the marine environment can, for instance, be influenced by strong selective forces, which are thought to increase under fishing and habitat fragmentation (Baskett et al. 2007). The models presented here could be used to study inert versus environmentally driven movements. The relative contribution of these two components of dispersal can have important consequences for metapopulation dynamics (Secor 1999, Clobert et al. 2009, Hawkes 2009), especially if heritable components of movements vary on short time-scales (Hawkes 2009). The transition matrix in an $\mathrm{HMM}$ can be interpreted as a habitat preference model for adult fish which actively choose habitats, or as a model for favourable conditions (whether actively chosen or not) for larval fish. Hierarchical and regression formulations within a hierarchical framework for underlying parameters could then help identify individual variation as opposed to inert behaviour and further relate these to individual condition or fitness. Otolith chemistry, in conjunction with other data, such as that issued from otolith structural analysis, can provide significant insights into the ecology of fish (Campana \& Thorrold 2001). I provide a structural framework that gives a probabilistic basis to analyse such complex data coherently. 
5.5 Discussion 


\section{Chapter 6}

\section{Inshore residency of fish larvae may maintain connections in a reef fish metapopulation}

\subsection{Introduction}

The lifecycle of most marine organisms displays a dispersive and elusive larval stage, which makes direct observation of dispersal between populations difficult and hinders efforts to predict dynamics of spatially structured populations. Despite an increasing number of studies, our knowledge of the relative importance of processes that govern larval dispersal remains limited. It is evident that larvae can, at least locally, determine the outcome of the dispersal process through behaviour and plasticity in their development (Bradbury et al. 2003, Lecchini 2005, Fiksen et al. 2007, Leis 2007, Morgan \& Anastasia 2008, Morgan \& Fisher 2010). The dynamic ocean environment through which larvae disperse may also play a predominant role by not only determining dispersal outcomes, but also adaptations of dispersing larvae to this environment (Armsworth et al. 2001, Strathmann et al. 2002, Largier 2003, Siegel et al. 2003, Shanks \& Eckert 2005, Byers \& Pringle 2006).

Many marine organisms must persist in advective environments, where the mean flow is unidirectional (Shanks \& Eckert 2005). In such environments, local hydrodynamic conditions are pivotal for the maintenance of populations at the upstream limit of the species: without 


\subsection{Introduction}

such mechanisms, populations would drift downstream and eventually go extinct (Swearer et al. 2002, Largier 2003, Byers \& Pringle 2006). Hydrodynamic conditions may therefore act as an important evolutionary driver of strategies to avoid advection and the associated uncertainty of returning to favourable settlement habitat (Strathmann et al. 2002, Shanks \& Eckert 2005). The successful self-recruitment of larvae to (or upstream of) their natal habitat depends on the relative strength of mean advective currents and the larval strategies to counter such advective forces (Armsworth 2001, Armsworth et al. 2001, Fisher 2005, Byers \& Pringle 2006). Larval behaviour may be particularly effective in that respect by allowing larvae to exploit retention features (Breitburg et al. 1995, Morgan \& Fisher 2010) or locally developing counter-currents (Paris \& Cowen 2004).

While the potential importance of such strategies for the maintenance of metapopulations and reserve networks is evident from theoretical models (White et al. 2010), the degree to which these strategies prevail on the scale of a metapopulation network is unknown. If adaptations to avoid advective forces dominate the dispersal process, we should find speciesspecific patterns of dispersal across the system, which could be reflected in common dispersal histories (i.e. adaptations for dispersal which cause the majority of larvae to migrate offshore (Morgan \& Anastasia 2008)). If, on the other hand, local hydrodynamic conditions determine dispersal histories, we would expect the dispersal histories of fish settling to differ according to site-specific conditions. By examining dispersal histories of settling fish in a number of local populations we may gain insight about the relative importance of drivers of dispersal patterns within a system.

Fish otoliths (ear-bones) have become an essential tool for fish biologists, allowing insights into dispersal histories in a variety of situations (Thorrold et al. 2002, Elsdon et al. 2008). In particular, the concentric 
growth and continued deposition of minerals during a fish's life make the otolith an ideal tool to study dispersal and migration: it acts as a 'flight recorder', continuously including trace elements that reflect environmental conditions (Campana 1999, Elsdon et al. 2008). Distinct chemical and visual marks in the otolith microstructure are furthermore associated with settlement and metamorphosis in some fish, facilitating identification of pelagic larval duration (PLD), settlement events and related metrics (Hale \& Swearer 2008, Hamilton \& Warner 2009).

Motivated by the seemingly contradictory result of upstream dispersal found in chapter 4, this chapter investigates dispersal histories of these Forsterygion lapillum recruits as evidenced by otolith chemistry. Describing dispersal histories from a series of chemical measurements is an active area of research and a number of statistical methods have recently been proposed to infer offshore transport (Sandin et al. 2005, Hamilton et al. 2008), describe habitat shifts (Fablet et al. 2007) or uncover patters from time-series of chemical measurements (or profiles / transects) across otoliths (Shima \& Swearer 2009a). In this chapter I build on the methods developed in chapter 5 to develop a flexible Bayesian model of larval dispersal in Cook Strait. I use a Bayesian mixture model that infers inshore residency from transects of chemical measurements across the recruit otoliths. Different wind-generated flow regimes simulated within the hydrodynamic model developed in chapter 4 provide insight into the potential benefit of inferred dispersal histories. I specifically aim to answer the following questions: i) given the hypothesis of two distinct water masses ('inshore and 'offshore'), do common triplefin larvae in Cook Strait disperse in offshore or nearshore waters? ii) Are these dispersal history patterns common to fish sampled throughout the system and iii) could these patterns be related to local hydrodynamics? 


\subsection{Methods}

\subsection{Methods}

\subsubsection{Recruit otolith preparation and pre - processing}

I investigated the dispersal history of $F$. lapillum recruits in three marine reserve sites (collected for chapter 4) using chemical profiles (time series of individual chemical scans) across otoliths. Chemical analysis of recruit otoliths followed methods described in Shima \& Swearer (2009a). Briefly, I used Laser Ablation Inductively Coupled Mass Spectrometry (LAICPMS) to sample material corresponding to the dispersive larval phase of recruits. Transects were ablated using a $80 \times 5 \mu \mathrm{m}$ rectangular spot which was moved across the post-rostral axis of the otolith at constant speed $(3 \mu \mathrm{m} / \mathrm{s})$. This produced a time series of elements across the otolith from just outside the natal (core) region to the most recently deposited material (i.e. the settlement mark). I determined the length of the transects visually by stopping transects at the settlement mark, which is visible in this species (Kohn 2007, Shima \& Swearer 2009a). I further used Barium as a chemical indicator of the settlement mark to guide this process (Hale \& Swearer 2008, Hamilton \& Warner 2009).

\subsubsection{Statistical model}

To characterize dispersal histories of recruits, I formulate a model that distinguishes inshore residence and offshore dispersal of larvae. The central assumption underlying this model and its interpretation is that some recruits will experience chemically distinguishable inshore and offshore waters. Inshore waters for instance may be water masses near a recruit's spawning location: F. lapillum spawns in sheltered locations close to shore, providing an empirical motivation for this assumption. I further assume that ontogenetic effects and differences in growth rate will have a negligible effect on overall patterns inferred by these models.

I start with the assumption that offshore water masses are usually depleted in some trace elements relative to inshore or estuarine waters. 
These waters may thus produce distinct chemical signatures in the otolith (this may not be the case for all elements or in regions of strong upwelling (Elsdon et al. 2008)). I restrict the elemental analysis to Barium, though other elements could be used and a multivariate extension of the method is straightforward. Barium to Calcium ratios in otoliths have been shown to be influenced by environmental concentrations (Walther \& Thorrold 2006), and are usually lower in oceanic waters relative to inshore waters (Elsdon \& Gillanders 2005, Elsdon et al. 2008, Hamilton et al. 2008). An example of Barium traces across F. lapillum recruit otoliths is shown in Figure 6.1.

At the most basic level, I model the Barium time series $\boldsymbol{y}_{\boldsymbol{i}}=y_{i, 1}, \ldots, y_{i, t}, \ldots, y_{i, T}$ of $T$ scans along the otolith of recruit $i$ as a mixture (a weighted sum) of $K$ normal distributions pertaining to the same number of potential source environments (Figure 6.1), each with different means $\mu=\mu_{1} \ldots \mu_{k} \ldots \mu_{K}$ and potentially unique standard deviations $\sigma=\sigma_{1} \ldots \sigma_{k} \ldots \sigma_{K}$. For $i=1 \ldots N$ fish the joint density is thus:

$$
f\left(\boldsymbol{y} \mid \boldsymbol{\pi}, \mu, \sigma^{2}\right)=\prod_{i=1}^{N} \prod_{t=1}^{T} \sum_{k=1}^{K} \pi_{i, k} f\left(y_{i, t} \mid \mu_{k}, \sigma_{k}^{2}\right)
$$

with $f$ denoting the normal density. $\pi_{i}=\left(\pi_{i}, 1 \ldots \pi_{i}, k \ldots \pi_{i}, K\right)$ and $\boldsymbol{\pi}=\left(\pi_{1} \ldots \pi_{i} \ldots \pi_{N}\right)$ then denote the vector over all source proportions for individual $i$, and the set of vectors of source proportions for all individuals, respectively. It is convenient to think of the $\pi_{i, k}$ as mixture proportions for the mixture components - 2 in this case, representing near-shore and offshore water masses.

For treatment in a Bayesian hierarchical sense, I re-write the model in terms of (latent) class indicator variables $\xi_{i, t}$ that assign the $t$-th observation of the $i$-th recruit to the $k$-th water mass: 


\subsection{Methods}

$$
\begin{aligned}
y_{i, t} \mid \mu, \sigma^{2}, \xi_{i, t} & \sim N\left(\mu_{1}+\xi_{i, t} \mu_{2}, \sigma_{\left(1+\xi_{i, t}\right)}^{2}\right) \\
\xi_{i, t} \mid \pi_{i} & \sim \operatorname{Bernoulli}\left(\pi_{i}\right) \\
\pi_{i, k} & \sim \operatorname{Beta}(1 / K)
\end{aligned}
$$

In its simplest form, this model will assign observations to one of two distributions, where $\mu_{1}$ is the mean of the offshore Barium signatures and $\mu_{1}+\mu_{2}$ is the mean of near-shore water masses (the additive formulation together with a restriction for $\mu_{2}$ to be positive is used for identifiability of the mixture indicators $\xi$, as their labels would otherwise lose their meaning during estimation). $\xi_{i, t}$ is thus either 0 for offshore or 1 for nearshore water masses and fish spend a proportion $\pi_{k}$ of their larval duration in environment $k$. This initial setup is similar to previous studies in terms of assumptions (Sandin et al. 2005, Hamilton et al. 2008) and model formulation (Fablet et al. 2007).
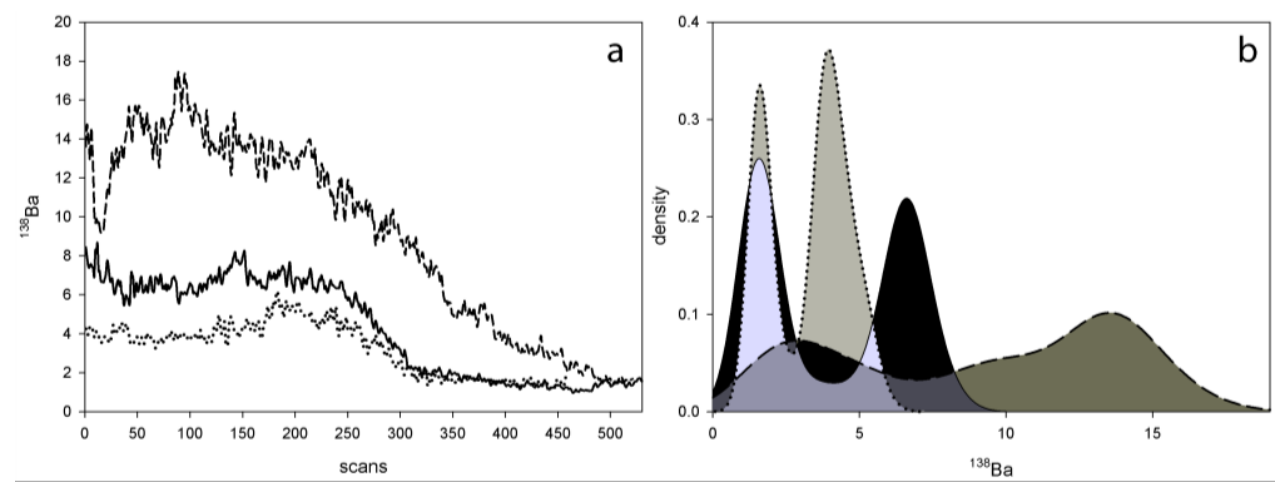

Figure 6.1: a) Example of three ${ }^{138} \mathrm{Ba}: \mathrm{Ca}$ traces in mol. $(\mathrm{mol} \mathrm{Ca})^{-1} \times 10^{-6}$ and b) resulting density estimates. Density outlines correspond to line types of the $\mathrm{Ba}$ traces; colours were added for ease of visualization of the densities. These densities are the basis of the mixture model which treats each transect as a mixture of two normal densities, one modelling near-shore water masses and the other modelling offshore water masses.

Many studies have used otolith chemistry for near-shore- and/or estuarine fish to distinguish natal regions (Warner et al. 2005, Barbee \& Swearer 2007, Ruttenberg et al. 2008, Standish et al. 2008, Fontes et al. 
2009, Neubauer et al. 2010). Estuaries may, for instance, have significantly higher Barium loads than coastal waters (Elsdon \& Gillanders 2005, Miller et al. 2010), which will in turn result in substantially higher Barium concentration for estuarine spawned individuals during their early life history. In terms of the above model, retention for some coastally spawned fish could then be under-estimated by falsely designating coastal inshore signatures as reflections of offshore water masses (see for instance the simulations in chapter 5). To incorporate the fact that different natal regions will produce different near-shore signatures, I extended this initial model to include individual random effects at this level:

$$
\begin{aligned}
y_{i, t} \mid \mu, \sigma^{2}, \xi_{i, t} & \sim N\left(\mu_{1}+\xi_{i, t} \mu_{i, 2}, \sigma_{\left(1+\xi_{i, t}\right)}^{2}\right) \\
\log \left(\mu_{i, 2}\right) & \sim N\left(\nu, \tau^{2}\right) \\
\xi_{i, t} \mid \pi_{i} & \sim \operatorname{Bernoulli}\left(\pi_{i}\right) \\
\pi_{i, k} & \sim \operatorname{Beta}(1 / K)
\end{aligned}
$$

The logarithm in this formulation of the random effect assures that nearshore water masses produce higher Ba signatures and makes the mixture identifiable. Priors for all quantities are vague.

Comparison of estimated values between sites may shed light on factors that govern dispersal in this system. For each site I calculated the posterior distribution of $\pi_{2}^{i}$, the proportion of time spent inshore by recruit $i$. For models applied independently to the three reserves, I also calculated the modal dispersal histories from the posterior distribution of $\xi$. Randomization tests were used to compare the pelagic larval duration (PLD) of recruits, expectations of retention proportions (the expected value of $\pi_{2}$ ) as well as total time dispersed $\tau=\pi_{1} * P L D$ amongst locations. I further investigated whether time spent offshore predicts the PLD. I again used randomization to assess significance of the regression coefficients that relate these two metrics. To verify that initial Ba did not account for the observed patterns in inshore vs. offshore residence (i.e. a strong 


\subsection{Methods}

ontogenetic signal) I used regression of maximum Ba concentrations for fish against $\pi_{2}$.

All results are based on 10000 samples from the posterior distribution after discarding the initial 1000 iterations for every Markov Chain (the burn-in). The resulting sequences of draws were inspected for autocorrelation and convergence. I tested whether the chains were sensitive to initial conditions by running the MCMC repeatedly for randomly generated initial values for parameters.

\subsubsection{Hydrodynamic model investigation}

In this chapter I again used an implementation of the Gerris flowsolver ocean model (outlined in Chapter 4) to investigate different flow patterns that may have developed during the period of investigation, namely from mid-December to early March 2008. I specifically studied differences in flow regimes induced by wind regimes, which are likely the driving force for local variation about the mean current (Heath 1986, Chiswell \& Stevens 2010). Such variation could be exploited by larvae of nearshore species to avoid advection in mean currents in order to stay near favourable habitats [Morgan, 2010 \#3562]. Two wind directions, northerly and southerly winds, make up the majority of observed winds in in Cook Strait (Figure 6.2). Recent empirical observations indicate that these winds can have a strong effect on dispersal of passive drifters in the vicinity of Kapiti Island (Chiswell \& Stevens 2010). Strong southerly winds can, for instance, drive drifter retention in Kapiti Island and may be enough to counter the mean flow induced by the d'Urville current.

I used information from regional wind records (obtained from the New Zealand National Climate Database at http://cliflo.niwa.co.nz/) to investigate wind regimes and corresponding flow patterns in the Strait. Winds were predominantly from the north (Figure 6.2a), but some prolonged southerly events also happened during the period 2 month 
period of this study (Figure 6.2b). I modelled distinct wind events comparable to those found during the time of our study as time averaged northerlies and southerlies which were compared against each other. Simulations were conducted over 2 and 5 day intervals, which is close to the mode and maximum of southerly wind event durations. I compared residual flow (by subtracting the tidal component of the flow from simulations) in the region between northerly and southerly regimes. Since empirical results where most intriguing for North Island populations, and because the effects of wind driven transport is easier to appreciate and visualize on a simple coastline, I concentrated this investigation on the stretch of coastline between Kapiti Island and Wellington. Gerris was allowed to refine the coastal topography and to adapt to flow patterns up to a maximum resolution of $117.19 \mathrm{~m}$ in this region, while keeping the resolution in the rest of Cook Strait at $3.5 \mathrm{~km}$. 


\subsection{Results}
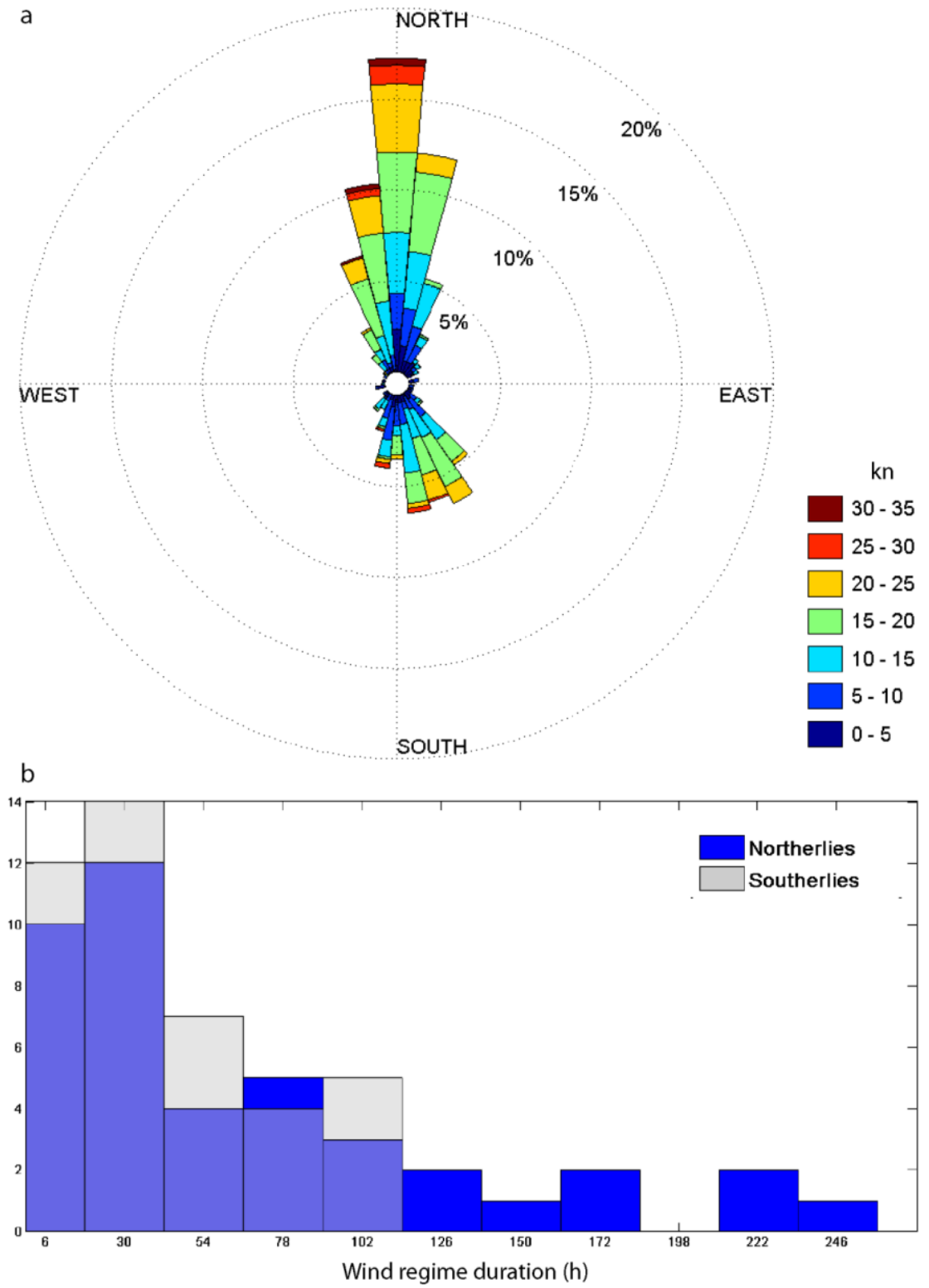

Figure 6.2: a) 6 hourly average wind direction and wind speed (in knots (kn)) at Wellington airport (obtained from the New Zealand National Climate Database at http://cliflo.niwa.co.nz/) during the time of the larval stage of recruits (midDecember 2007 - March 2008). b) Histogram of the frequency of wind regime durations, defined by constant wind direction over a number of 6hour intervals.

\subsection{Results}

\subsubsection{Results from models of dispersal histories}

When considering the application of the mixture model for the collection sites, some striking differences appear (Table 6.1). Offshore 
means $\left(\mu_{1}\right)$ are considerably higher in Long Island relative to Wellington and Kapiti offshore signatures. Differences between mean inshore $(\log (\nu))$ and offshore signatures are also greatest at Long Island, whereas recruits at Kapiti show the most subtle changes in Ba. Signatures are also the least variable in Kapiti, whereas both inshore means and their variance are substantially higher in Long Island, and to a lesser degree in Wellington. Offshore means are rather similar between Kapiti and Wellington recruits; however the variance about this mean is considerably higher in Wellington. The variance around individual inshore means is indistinguishable between the two sites but the variance about the random effects mean is considerably higher in Wellington, owing to the large spread in inshore signatures between self-recruited individuals, presumably from Wellington harbour (c.f. chapter 4), and dispersed individuals from Kapiti (see results below).

Table 6.1: Expected values and 95 per cent credible interval (in square brackets) of the posterior distribution of some parameters in the dispersal model. Values are in mol Ba. mol $\mathrm{Ca}^{-1}\left(\times 10^{-6}\right) . \mu_{1}$ designates the offshore water-mass mean, $\sigma_{1}^{2}$ its variance. The variance in of individual water masses is given by $\sigma_{2}^{2}$. The mean of the random effect for near-shore water-masses is given by $\mu_{1}+\log (\nu)$ with associated variance $\varsigma^{2}$ for the random effect.

\begin{tabular}{c|c|c|c|c|c|} 
Site & $\mu_{1}$ & $\log (\nu)$ & $\sigma_{1}^{2}$ & $\sigma_{2}^{2}$ & $\varsigma^{2}$ \\
\hline \multirow{2}{*}{ Kapiti } & 1.18 & 2.03 & 0.52 & 0.16 & 0.47 \\
& {$[1.16 ; 1.20]$} & {$[1.73 ; 2.34]$} & {$[0.50 ; 0.54]$} & {$[0.14 ; 0.17]$} & {$[0.24 ; 0.91]$} \\
\hline Island & 1.07 & 3.29 & 2.07 & 0.16 & 4.85 \\
Bay & {$[1.06 ; 1.09]$} & {$[2.38 ; 4.19]$} & {$[2.00 ; 2.14]$} & {$[0.15 ; 0.17]$} & {$[2.56 ; 9.20]$} \\
\hline Long & 5.62 & 6.56 & 4.60 & 9.76 & 40.69 \\
Island & {$[5.48 ; 5.75]$} & {$[3.87 ; 9.23]$} & {$[4.37 ; 4.83]$} & {$[8.93 ; 10.57]$} & {$[20.33 ; 80.25]$} \\
\hline
\end{tabular}

Dispersal patterns are similar at all sites, showing substantial propositions of retention signatures for earlier parts of the PLD of most larvae (Figure 6.3). The posterior expectation for the retention proportion 


\subsection{Results}

$\pi_{2}$ ranged from 0.41 to 0.98 in Kapiti (Figure 6.3a), from 0.02 to 0.98 in Island Bay (Figure 6.3b) and from 0.26 to 0.99 in Long Island (Figure 6.3c). Only in Island Bay do I find four larvae whose dispersal history indicates that they likely spent all of their larval life in waters masses characterized as offshore. This seems to be the case for only one recruit in Long Island and none in Kapiti Island.

When comparing dispersal histories to predicted self-recruitment patterns (see chapter 4), results suggest that most self-recruited fish were retained inshore for the majority or all of their PLD. These results are further consistent with the hypothesis that retained individuals in Long Island were retained within the Sound for their entire PLD (Figure 6.3c). A similar pattern is evidenced for fish that were regionally self-recruited in Island Bay: I hypothesized that most of these fish likely came from within the harbour in Wellington (c.f. chapter 4), and dispersal histories are consistent with this hypothesis. Model results suggest that many of them appear to have spent the majority of their PLD within Wellington Harbour, or surrounding bays, before recruiting to the nearby open coast (Figure 6.3b). Some, however, appear to have been flushed out of the harbour earlier, yet they still recruited close to their natal region. The dispersal model also predicts that recruits at Kapiti likely spent most of their PLD inshore, though one individual appears to have left inshore waters for offshore waters before recruiting back to the natal region (Figure 6.3a).

The Pelagic larval duration is not significantly different amongst any of the locations (Table 6.2). Time and proportion of PLD spent nearshore is higher at Kapiti than at either of the other locations, though these patterns are only marginally significant. Furthermore, this pattern is driven by the few offshore dispersers at Long Island and Wellington: leaving out dispersers in these tests leaves the pairwise tests non-significant (Table 6.2). Regressions of PLD versus $\lambda$ and $\tau$ did not show significant 
associations between PLD and either of these parameters ( $r^{2}=4.76 e^{-4}, p=0.41$ and $r^{2}=0.0127, p=0.18$ respectively). Maximum barium concentrations were not related to time spent inshore $\left(r^{2}=0.00059, p=0.56\right)$.

Table 6.2: Pairwise p-values from randomization tests after 20000 iterations. Values in the numerator of the fraction are p-values for tests with all recruits, values in the denominator are p-values with dispersers $\left(E\left(\pi_{1}\right)>0.9\right.$ for Wellington and $E\left(\pi_{1}\right)>0.74$ for Long Island) omitted from the tests. PLD is the pelagic larval duration, $\pi$ denotes the proportion of time spent in near-shore water masses and $\tau$ the time spent in offshore water-masses.

\begin{tabular}{l|ccc|ccc|} 
& \multicolumn{3}{|c|}{ Wellington } & \multicolumn{3}{c|}{ Long Is. } \\
\hline & PLD & $\pi$ & $\tau$ & PLD & $\pi$ & $\tau$ \\
\hline Kapiti & 0.30 & $\frac{0.062}{0.50}$ & $\frac{0.11}{0.34}$ & 0.22 & $\frac{0.064}{0.12}$ & $\frac{0.083}{0.13}$ \\
Wellington & - & - & - & 0.51 & 0.54 & 0.53 \\
\hline
\end{tabular}

\subsubsection{Hydrodynamic modelling results}

In order to explore a hydrodynamic explanation of inferred dispersal patterns and their potential benefit, I examined flow patterns that develop in average northerly and southerly conditions in Cook Strait. Simulations of northerly versus southerly wind events indicate that sustained southerly winds have the potential to produce sustained counter-currents in residual flow along the western coast of New Zealand's north island (Figure 6.4). Residual flow during the predominant northerly conditions is shown to be south-eastward through Cook Strait and in the vicinity of Kapiti Island (Figure $6.4 a, c)$. Though often disrupted by eddies formed by flow along the complex coastline of the Marlborough Sound, this mean flow is accentuated by persistent northerly conditions, resulting in strong unidirectional flow through the strait (Figure 6.4c). 

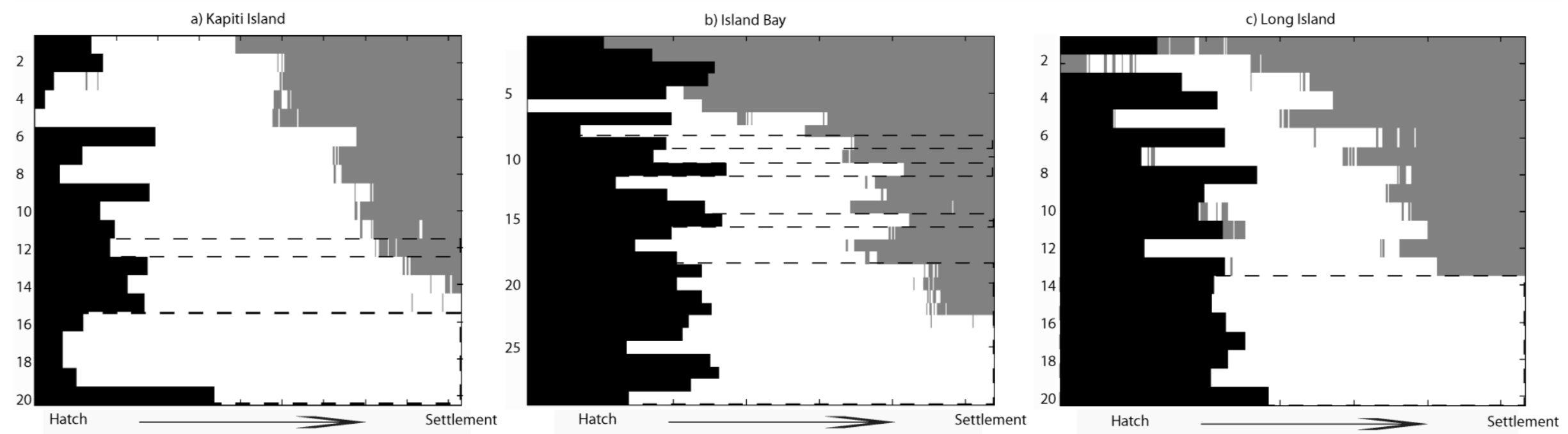

Figure 6.3: Modal estimated dispersal patterns of all fish (individual lines, identified by numbers on the y-axis) at each site, sorted by number of frames with inshore signatures (white) and aligned at the settlement mark. Offshore signatures appear in grey, black frames indicate absence of data (there is considerable plasticity in PLDs between fish). Dashed boxes indicate fish that were predicted to have self-recruited in chapter 4. 


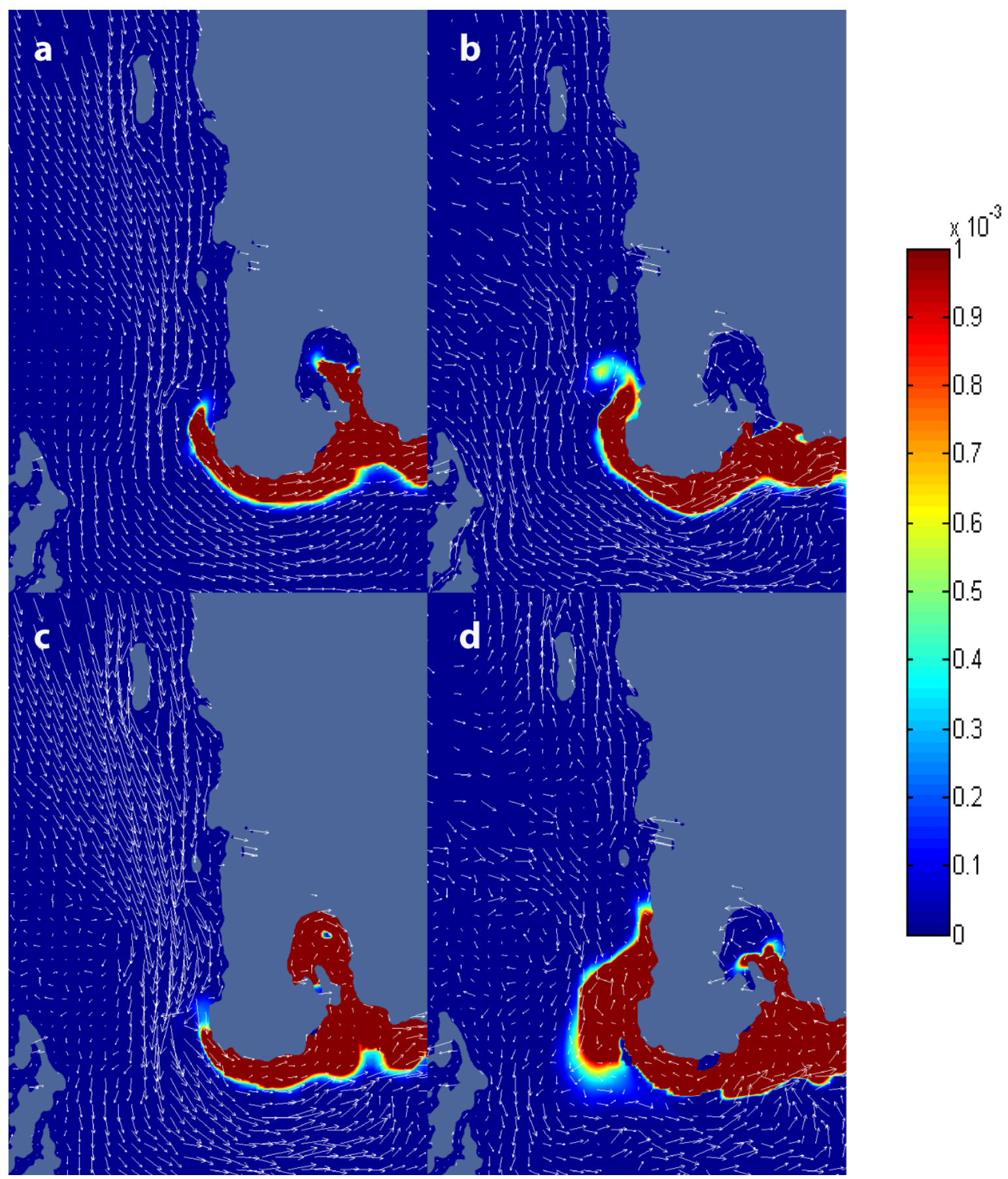

Figure 6.4: Visualization of Gerris simulation results (refined to $\sim 468.76 \mathrm{~m}$ resolution for visualization purposes) of flow velocity (arrows) and the passive tracer field (red) in Cook Strait during persistent, time averaged $(a, c)$ northerly and $(b, d)$ southerly wind events. $(a, b)$ Flow and tracer distribution after two days and $(c, d) 5$ days of persistent wind conditions. 


\subsection{Discussion}

Persistent southerly conditions have the potential to reverse the residual flow on the eastern border of the strait, a flow regime which gains in breadth and velocity with on-going southerlies (Figure 6.4b,d). This flow feature persists up the western coast of the North Island, past Kapiti Island. Passive tracers are entrained in this residual flow along the coastline. Some however get dispersed into the south-easterly mean flow in the strait, often by eddies which form off the many headlands on the South eastern border of the strait (Figure 6.4b,d).

\subsection{Discussion}

The stability and dynamics of marine metapopulations depend critically on dispersal patterns within the network of local populations (Armsworth 2002, Hastings \& Botsford 2006, Rozenfeld et al. 2008). These connections may be influenced by regional hydrodynamic features (James et al. 2002, White et al. 2010) as well as biological features of the dispersing organism and post-settlement processes. A number of such biological features may influence dispersal and post settlement processes, such as larval swimming and sensory abilities (Kingsford et al. 2002, Fisher 2005, Lecchini et al. 2005, Gerlach et al. 2007, Leis 2007), larval behaviour (Bradbury et al. 2003, Fiksen et al. 2007, Morgan \& Anastasia 2008, Morgan \& Fisher 2010) as well as differential fitness induced by maternal effects and the dispersive environment (Marshall et al. 2008, Shima \& Swearer 2009a, Marshall et al. 2010).

These, often species specific features, are the result of selective processes which act to maintain local populations. Factors such as fragmentation of populations may cause adaptations and selection of dispersal strategies depending on the configuration of populations and habitat within the dispersive ocean matrix (e.g. Baskett et al. 2007). The interplay of local environmental conditions and their evolutionary and 
ecological consequences for dispersal in the sea has rarely been studied on the scale of a metapopulation (but see Diehl et al. 2007). The aim of this study was to gain insights from fish otoliths and hydrodynamic simulations into the factors that likely shape predicted dispersal patterns in $F$. lapillum in Cook Strait. Considerable similarity in dispersal patterns between sites suggest that larvae remain close to shore for much of their PLD, suggesting species-specific adaptations to a dispersive habitat.

\subsubsection{Mixture model results for dispersal histories}

Modelling results are naturally conditional on assumptions being at least approximately true. The biggest assumption made in this study is that inferred patterns indeed describe a difference in water masses and not some ontogenetic or otherwise physiologically induced pattern. Ba has been shown to be ontogenetically enriched in the core of otoliths of many species (Ruttenberg et al. 2005) and may be influenced by maternal effects (Thorrold et al. 2006). These effects are confined to the direct vicinity of the core however (e.g. $<50 \mu \mathrm{m}$ from the core for enriched isotopes (Thorrold et al. 2006)). Additionally, strong plasticity of the dispersal histories evidenced by the model and their consistency with predicted selfrecruitment and dispersal pathways makes this an unlikely explanation. Ba in traces was consistently higher in traces of fish predicted to have come from enclosed environments, where freshwater runoff can lead to decreased salinity and increased Ba concentrations, two factors which positively influence $\mathrm{Ba}$ concentrations found in otoliths (Walther \& Thorrold 2006). There may be a 'washout' effect, where residual material contained in the sample induction system of the LA-ICPMS causes the signal to diminish more slowly than actual decreases in Ba. The high frequency of sampling should however lead to a rapid washout and this may be less of a concern. Furthermore, initial Ba was not correlated with the proportion of the PLD spent inshore, as would be expected if patterns were due to washout effects. 


\subsection{Discussion}

I found considerable differences in nearshore and offshore signatures between sites, especially between recruits collected at the Long Island and recruits found on North Island sites. These patterns are interpretable however, and they may even help infer levels of variation of Barium concentrations in water masses of different regions in Cook Strait and thereby identify water masses which larvae were situated in while dispersing.

Kapiti island is flushed by currents originating in the d'Urville current (Chiswell \& Stevens 2010) and it is therefore expected that any differences in $\mathrm{Ba}$ concentrations found in this environment are subtle with respect to overall differences in the whole system. Furthermore, about half of the recruits to Kapiti Island were predicted to have come from Wellington. Low $\mathrm{Ba}$ of inshore signatures indicates that these individuals potentially dispersed along the open coast and did not remain in Wellington waters, characterized by higher $\mathrm{Ba}$ (Table 1). This is consistent with a scenario in which these recruits dispersed with the counter currents developing in southerly conditions along the western Cook Strait, where $\mathrm{Ba}$ concentrations differences may be equally minute.

Recruits collected at Long Island rarely experience water masses with such low Ba during their larval history. 'Offshore' waters in Long Island are considerably different from those in Kapiti Island and Wellington. They may be influenced by waters originating from the sounds themselves, and this reflection in the offshore signatures of recruits at Long Island suggests that larvae may not disperse far offshore in this region but rather stay within water masses originating from the Sounds. In fact, even signatures characterized as 'offshore' in Long Island recruits are high in Ba relative to other studies whereas Wellington and Kapiti offshore signatures are similar to values usually found for ocean waters (e.g. Miller et al. 2010). 
Recruits at this location thus provide additional evidence that dispersal may predominantly happen close to shore for F. lapillum.

\subsubsection{Interpreting dispersal patterns}

Based on the inferred quantities from the dispersal model and conditional on the assumptions of the model, there is evidence of striking similarities in the dispersal histories of settling fish at the three sites. In particular, a large majority of fish appear to remain inshore for the early portion of their PLD, irrespective of the site. Cook Strait is notorious for its strong winds as well as dangerous tidal and wind driven currents (i.e. Heath 1986) and may be thought of as a highly dispersive environment. While the inferred inshore residency during the early part of the PLD may be the result of hydrodynamic conditions and spawning in sheltered sites, the predicted occurrence of similar dispersal histories of dispersed fish at all sites makes this explanation unlikely, especially with respect to the predicted upstream dispersal of larvae from Wellington to Kapiti (see chapter 4).

One intuitive explanation of the inshore residency is that successful settlement of larvae depends on staying inshore for early parts of the PLD. This could be related to higher fitness and thus lower mortality of larvae which encounter favourable conditions (i.e. high food availability), which may occur more frequently in inshore water masses, and in particular in enclosed bays (Shima \& Swearer 2009a, 2010, Swearer \& Shima 2010). Swimming abilities of fish larvae are often substantial during the latter part of their PLD (Fisher 2005, Leis 2007), which may lead to increased foraging skills and abilities to actively swim against strong currents. The offshore component predicted later in the dispersive phase of these larvae may then correspond to a loss of a net fitness gain of inshore waters later in the dispersive phase. 


\subsection{Discussion}

An alternative explanation for these dispersal history patterns is in terms of evolutionary constraints. A growing body of research suggests that dispersal may be a by-product of the pelagic larval phase of most marine organisms, and that adaptations during this phase may be geared towards self-recruitment (Strathmann et al. 2002, Byers \& Pringle 2006). Especially in advective flow regimes, such adaptations need to be strong enough to allow the maintenance of upstream populations (Byers \& Pringle 2006) and recent research highlights a number of species-specific traits that may allow larvae to exploit local current features in order to remain close to or return to suitable habitat (Kingsford et al. 2002, Paris \& Cowen 2004, Shanks \& Eckert 2005, Gerlach et al. 2007, Shanks \& Kipp Shearman 2009, Morgan \& Fisher 2010). The availability of suitable habitat, especially of sheltered bays conducive to spawning, may be limited along much of the coast, and especially downstream of Cook Strait (Smith 2008). This may provide an incentive for early stage larvae, which often have limited sensory and swimming abilities (Kingsford et al. 2002, Leis 2007), to stay close to shore(Strathmann et al. 2002).

In Cook Strait, the inshore residency of larvae may permit not only selfrecruitment but also the use of wind driven current features, thereby permitting upstream connections. This is evident in the predicted dispersal of larvae from Wellington to Kapiti Island, presumably by coastal countercurrents driven by southerly wind conditions. Local populations of $F$. lapillum around the upstream island of Kapiti are in relatively low abundance (Smith 2008) and consist of small patches along the eastern site of the island (P.Neubauer, pers.obs.), which are unlikely to be selfsustaining (in fact, many of these patches appear and disappear over short time-scales, P.Neubauer, pers.obs.). Most of the spawner biomass for this species is located in the Marlborough Sounds and Wellington Harbour (Smith 2008), and dispersal patterns may have evolved to sustain upstream local populations by both self-recruitment and supply of recruits to 
upstream sinks. If the latter is a simple coincidental consequence of an adaptation to self-recruitment, then F. lapillum populations at Kapiti may be an 'accidental sink' produced by the specific current reversals in the western Cook Strait.

\subsubsection{Conclusions}

Uncovering the mechanisms that underlie the structure of populations is of importance for spatial management of marine resources and their conservation. Larval dispersal between marine reserves and from reserves to fished areas are two appealing features of marine reserve networks in the context of conservation and fisheries management (Botsford et al. 2001, Roberts et al. 2001). Dispersal in such systems is likely to depend on a range of biological, physical and evolutionary factors, and estimates of connectivity are typically associated with large uncertainty (Halpern et al. 2006). Demonstrating which factors govern connections and selfrecruitment patterns in a network is therefore pivotal in the development of accurate dispersal estimates.

This study sheds some light on the potential mechanisms which allow dispersal and maintenance of local F. lapillum populations in Cook Strait. While the simulations employed in this chapter are abstract illustrations of current variability under different wind regimes, empirical results and simulation outcomes combined provide strong evidence that dispersal of F.lapillum in Cook Strait may largely happen away from the relatively invariable geostrophic mean currents in Cook Strait. This in turn suggests that occasional nearshore current reversals may have a disproportionate effect on dispersal outcomes, and could lead to upstream connectivity on ecological timescales. While the factors affecting dispersal patterns and the evolution of dispersal strategies are likely species specific, species with similar life histories may be affected by similar environmental and selective forces and may thus respond in similar ways (i.e. Shanks \& Eckert 2005) 


\subsection{Discussion}

and insights about representative species may help explain observations or predict dispersal of species with similar life histories. 


\section{Chapter 7}

\section{Discussion and perspectives}

The aim of this thesis was to investigate the use of new statistical and hydrodynamic simulation methods for inference of population connectivity of $F$. lapillum from otolith chemical signatures. In developing these methods, I sought to address and improve upon a number of shortcomings inherent in both otolith chemical approaches and the statistical methods commonly used to analyse this geochemical data. The solutions proposed in this thesis may be particularly useful for marine applications, where the use of such methods often poses logistical and inferential problems.

Gaining a reliable snapshot, let alone a long term picture of inter-patch connectivity, has been a major challenge in marine metapopulations. Even with sophisticated tracking methods such as geochemical fingerprints or paternity testing, one would have to track propagules from all patches to their destination in order to gain a complete understanding of connections in a metapopulation. Unless a species spawns and settles in very distinct areas, this will often not be realistically possible or logistically feasible. Tracking approaches can, however, shed light on realized connections and the mechanism behind such connectivity (Thorrold et al. 2001, Thorrold et al. 2002, Almany et al. 2007, Planes et al. 2009). The mere existence of such connections, beyond their potential ecological importance, has evolutionary implications as a genetically homogenizing force and potential source of recruits for extinct patches. Such connections may therefore not only drive metapopulation dynamics but also guarantee the persistence of 


\section{Discussion and perspectives}

a metapopulation as a whole (Botsford et al. 2001, Armsworth 2002, Hill et al. 2002, Hastings \& Botsford 2006, Campbell Grant et al. 2010).

Developing new Bayesian approaches for tracking of fish larvae in the marine environment was an essential part of my thesis, which allowed me to make inferences about realized connections amongst local F. lapillum populations and potential mechanisms of evidenced connectivity in Cook Strait. Coupling tracking methods to hydrodynamic simulations lent credibility to connections predicted from otolith chemistry, and, vise-versa, finding such connections indicated that mean field hydrodynamics are a valuable predictor of some connections. The simulation carried out for chapter 4 also revealed discrepancies between predicted connectivity and mean flows in Cook Strait, and motivated a more refined set of simulations and a closer look at dispersal histories of F. lapillum recruits. This study, in turn, evidenced patterns in dispersal histories across all sites that potentially represent species-specific adaptations to the highly dispersive ocean matrix in and around Cook Strait. Combining and contrasting simulation and empirical estimates of connectivity can thus provide considerable insight into patterns that drive connectivity in a metapopulation. Building such mechanistic understanding of dispersal may ultimately lead to a better understanding of marine metapopulation dynamics and could thus help to better design spatial management and conservation approaches.

The statistical methods presented in this thesis are a step towards greater applicability of otolith chemical approaches in oceanic environments. Specifically, the potential presence of extra baseline sources in the sample of recruits or a mixed fisheries sample has thus far posed problems when applying otolith chemistry in marine settings. In this respect, the exclusion method presented in chapter 1 is likely of greater value for exploratory purposes or for specific ecological scenarios than for 
overall connectivity studies. The exclusion test provides an easy yet powerful method for simple questions such as import or export of larvae from a marine reserve. However, this test is shown to perform poorly on larger scales, and estimates of its exclusion potential are strongly dependent on the sampled proportion of the overall variability of chemical signatures in a system.

Dirichlet process mixtures (DPM) are shown to perform well in simulations and can detect extra baseline samples if these are sufficiently different from the sampled geochemical atlas. On the opposite end of the spectrum, the application to weakfish in chapter 3 showed that if most fish likely came from extra baseline sources (or if sampling methods differ between mixture and baseline, and the data is slightly 'off'), there may be little information about dispersal to be gained from an application of the DPM model alone. If appropriate, the DPM classification procedure can be considerably more informative in such situations. However, this classifier may not always be appropriate. For species with a widespread distribution and potential long distance dispersal, only a range-wide sampling scheme would allow for such classification to be performed in a meaningful way. But in such scenarios it may well be that otolith geochemistry reaches the limits of its resolving power on scales far smaller than the distributional range of the species. DPM based methods can thus provide a valuable tool in many situations in which it is difficult or impossible to construct a complete atlas. They do not, however, present a quick fix method which does away with fundamental problems that are inherent in otolith chemical approaches, such as low resolution or low coverage of the chemical variability found in the dispersal range of a species by a (relatively) small baseline sample.

Dispersal histories may be more telling about mechanisms that underlie predicted dispersal patterns, and chapter 5 outlines a range of 


\section{Discussion and perspectives}

possibilities to model dispersal patterns and to choose appropriate models. While chapter six provides an arguably simplistic application of this modelling framework, the ability to detect common dispersal patterns (or contingents) and to quantify their similarity is a powerful tool for dispersal studies. Shanks \& Eckert (2005), for instance, hypothesized that fish with different habitat preferences with respect to distance from shore may have evolved to similarly exploit current variability and up/downwelling regimes along the Californian coast. Provided these water masses are chemically distinguishable (i.e. high Barium in upwelled waters), one could test such a hypothesis with a representative multi-species dataset and assess whether similarities in dispersal histories exist for species with similar niches.

A valuable extension of the present work would be to investigate the importance of the connectivity patterns for population dynamics of $F$. lapillum. Recent work by Shima \& Swearer, for instance, indicates that fitness and thus the successful recruitment of $F$. lapillum depends strongly on their dispersal histories (Shima \& Swearer 2009a, Shima \& Swearer 2009b, Shima \& Swearer 2010, Swearer \& Shima 2010). Dispersers may thus be selected against, thereby dampening the importance of dispersal for population maintenance. Similarly, phenotype-environment mismatches for larvae which dispersed but are not adapted to the new environment may markedly lower the importance of such connections for local populations (Marshall et al. 2010). Assessing the importance of individual dispersal pathways to overall population connectivity is a daunting task since it involves not only finding these pathways but also following dispersed cohorts throughout their life and assessing their reproductive output. Nevertheless such studies are possible in manageable systems and may be increasingly manageable in other systems for which dispersal pathways have been identified (i.e. Almany et al. 2007, Planes et al. 2009). 
Even if the relative importance of dispersers for population dynamics may be relatively low, the rescue potential of these connections, however, can be of vital importance on evolutionary time-scales (Hill et al. 2002, Campbell Grant et al. 2010). Although dispersed recruits may be of lower fitness and have higher mortality or lower reproductive output, environmental changes or catastrophic events can reverse the odds over long time scales, and these connections would then help maintain populations. This is of considerable importance for conservation and management of marine resources, where the desired outcome is often the long term viability of a resource or a depleted population (Botsford et al. 2001, Fogarty \& Botsford 2007). 


\section{Bibliography}

Almany GR, Berumen ML, Thorrold SR, Planes S, Jones GP (2007) Local replenishment of coral reef fish populations in a marine reserve. Science 316:742-744

Anderson MJ (2001) A new method for non-parametric multivariate analysis of variance. Austral Ecol 26:32-46

Anderson MJ, Gorley RN, Clarke KR (2008) PERMANOVA+ for PRIMER: Guide to Software and Statistical Methods. PRIMER-E, Plymouth, UK

Arkema KK, Abramson SC, Dewsbury BM (2006) Marine ecosystem-based management: from characterization to implementation. Front Ecol Environ 4:525-532

Armsworth PR (2001) Directed motion in the sea: efficient swimming by reef fish larvae. J Theor Biol 210:81-91

Armsworth PR (2002) Recruitment limitation, population regulation, and larval connectivity in reef fish metapopulations. Ecology 83:10921104

Armsworth PR, James MK, Bode L (2001) When to press on or turn back: dispersal strategies for reef fish larvae. Am Nat 157:434-450

Artzy-Randrup Y, Stone L (2010) Connectivity, Cycles, and Persistence Thresholds in Metapopulation Networks. PLoS Comput Biol 6:e1000876

Ashford J, La Mesa M, Fach BA, Jones C, Everson I (2010) Testing early life connectivity using otolith chemistry and particle-tracking simulations. Can J Fish Aquat Sci 67:1303-1315

Avasthi A (2005) Ecosystem management - California tries to connect its scattered marine reserves. Science 308:487-488

Barbee NC, Swearer SE (2007) Characterizing natal source population signatures in the diadromous fish Galaxias maculatus, using embryonic otolith chemistry. Mar Ecol Prog Ser 343:273-282

Barnett-Johnson R, Pearson TE, Ramos FC, Grimes CB, MacFarlane RB (2008) Tracking natal origins of salmon using isotopes, otoliths, and landscape geology. Limnol Oceanogr 53:1633-1642

Baskett ML, Weitz JS, Levin SA (2007) The evolution of dispersal in reserve networks. Am Nat 170:59-78

Becker BJ, Levin LA, Fodrie FJ, McMillan PA (2007) Complex larval connectivity patterns among marine invertebrate populations. Proc Natl Acad Sci U S A 104:3267-3272

Berumen ML, Walsh HJ, Raventos N, Planes S, Jones GP, Starczak V, Thorrold SR (2010) Otolith geochemistry does not reflect dispersal history of clownfish larvae. Coral Reefs 29:883-891

Black KP, Moran PJ (1991) Influence of hydrodynamics on the passive dispersal and initial recruitment of larvae of Acanthaster planci 
(Echinodermata: Asteroidea) on the Great Barrier Reef. Mar Ecol Prog Ser 69:55-65

Bohnsack JA (1998) Application of marine reserves to reef fisheries management. Aust J Ecol 23:298-304

Bolker BM, Okuyama T, Bjorndal KA, Bolten AB (2007) Incorporating multiple mixed stocks in mixed stock analysis: 'many-to-many' analyses. Mol Ecol 16:685-695

Botsford LW (2005) Potential contributions of marine reserves to sustainable fisheries: Recent modeling results. Bull Mar Sci 76:245259

Botsford LW, Castilla JC, Peterson CH (1997) The management of fisheries and marine ecosystems. Science 277:509-515

Botsford LW, Hastings A, Gaines SD (2001) Dependence of sustainability on the configuration of marine reserves and larval dispersal. Ecol Lett 4:144-150

Bowman MJ, Kibblewhite AC, Murtagh RA, Chiswell SM, Sanderson BG (1983) Circulation and mixing in greater Cook Strait, New Zealand. Oceanol Acta 6:383-391

Bradbury IR, Campana SE, Bentzen P (2008) Estimating contemporary early life-history dispersal in an estuarine fish: integrating molecular and otolith elemental approaches. Mol Ecol 17:1438-1450

Bradbury IR, Snelgrove PVR, Pepin P (2003) Passive and active behavioural contributions to the patchiness and spatial pattern during the early life history of marine fishes. Mar Ecol Prog Ser 257:233245

Breitburg DL, Palmer MA, Loher T (1995) Larval distributions and the spatial patterns of settlement of an oyster reef fish: responses to flow and structure. Mar Ecol Prog Ser 125:45-60

Brophy D, Jeffries TE, Danilowicz BS (2004) Elevated manganese concentrations at the cores of clupeid otoliths: possible environmental, physiological, or structural origins. Mar Biol 144:779-786

Burkard RE, Rendl F (1984) A thermodynamically motivated simulation procedure for combinatorial optimization problems. European Journal of Operational Research 17:169-174

Burton RK, Koch PL (1999) Isotopic tracking of foraging and long-distance migration in northeastern Pacific pinnipeds. Oecologia 119:578-585

Byers J, E., Pringle JM (2006) Going against the flow: retention, range limits and invasions in advective environments. Mar Ecol Prog Ser 313:27-41

Caley MJ, Carr MH, Hixon MA, Hughes TP, Jones GP, Menge BA (1996) Recruitment and the local dynamics of open marine populations. Annu Rev Ecol Syst 27:477-500

Campana SE (1999) Chemistry and composition of fish otoliths: pathways, mechanisms and applications. Mar Ecol Prog Ser 188:263-297

Campana SE (2005) Otolith science entering the 21st century. Mar Freshw Res 56:485-495 
Campana SE, Chouinard GA, Hanson JM, Frechet A, Brattey J (2000) Otolith elemental fingerprints as biological tracers of fish stocks. Fisheries Research 46:343-357

Campana SE, Thorrold SR (2001) Otoliths, increments and elements: keys to a comprehensive understanding of fish populations? Can J Fish Aquat Sci 58:30-38

Campana SE, Valentin A, Sevigny DM, Power D (2007) Tracking seasonal migrations of redfish (Sebastes spp.) in and around the Gulf of St. Lawrence using otolith elemental fingerprints. Can J Fish Aquat Sci 64:6-18

Campbell Grant EH, Nichols JD, Lowe WH, Fagan WF (2010) Use of multiple dispersal pathways facilitates amphibian persistence in stream networks. Proceedings of the National Academy of Sciences 107:6936-6940

Camus PA, Lima M (2002) Populations, metapopulations, and the openclosed dilemma: the conflict between operational and natural population concepts. Oikos 97:433-438

Cappé O, Moulines E, Rydén T (2005) Inference in hidden Markov models, Vol. Springer, New York ; London

Casella G, Robert CP (1996) Rao-Blackwellisation of sampling schemes. Biometrika 83:81-94

Chevenet F, Brun C, Banuls A-L, Jacq B, Christen R (2006) TreeDyn: towards dynamic graphics and annotations for analyses of trees. BMC Bioinformatics 7:439

Chib S (1995) Marginal Likelihood from the Gibbs Output. J Amer Statistical Assoc 90:1313-1321

Chiswell SM, Stevens CL (2010) Lagrangian and Eulerian estimates of circulation in the lee of Kapiti Island, New Zealand. Cont Shelf Res 30:515-532

Chittaro PM, Hogan JD, Gagnon J, Fryer BJ, Sale PF (2006) In situ experiment of ontogenetic variability in the otolith chemistry of Stegastes partitus. Mar Biol 149:1227-1235

Clark CW (1996) Marine reserves and the precautionary management of fisheries. Ecol Appl 6:369-370

Clark JS (2003) Uncertainty and variability in demography and population growth: a hierarchical approach. Ecology 84:1370-1381

Clements KD (2003) Triplefins. In: Andrew N, Francis M (eds) The Living Reef: The Ecology of New Zealand's Rocky Reefs. Craig Potton Publishing, Nelson, p 160-167

Clobert J, Le Galliard J-F, Cote J, Meylan S, Massot M (2009) Informed dispersal, heterogeneity in animal dispersal syndromes and the dynamics of spatially structured populations. Ecol Lett 12:197-209

Cornuet JM, Piry S, Luikart G, Estoup A, Solignac M (1999) New methods employing multilocus genotypes to select or exclude populations as origins of individuals. Genetics 153:1989-2000

Cowen R, Lwiza KMM, Sponaugle S, Paris CB, Olson DB (2000) Connectivity of marine populations: open or closed? Science 287:857-859 
Cowen RK, Gawarkiewic G, Pineda J, Thorrold SR, Werner FE (2007) Population Connectivity in Marine Systems An Overview. Oceanography 20:14-21

Cowen RK, Paris CB, Srinivasan A (2006) Scaling of connectivity in marine populations. Science 311:522-527

Crooks KR, Sanjayan MA (2006) Connectivity conservation, Vol. Cambridge University Press, Cambridge :

Cudney-Bueno R, Lavin MF, Marinone SG, Raimondi PT, Shaw WW (2009) Rapid Effects of Marine Reserves via Larval Dispersal. PLoS ONE 4

Cushing DH (1990) Plankton production and year-class strenght in fish populations - an update of the match mismatch hypothesis. Adv Mar Biol 26:249-293

Dahl DB (2003) An improved merge-split sampler for conjugate Dirichlet process mixture models. Report No. \#1086, University of Wisconsin-Madison . Madison, Wisc

Dahlgren CP, Eggleston DB (2000) Ecological processes underlying ontogenetic habitat shifts in a coral reef fish. Ecology 81:2227-2240

Dawson KJ, Belkhir K (2001) A Bayesian approach to the identification of panmictic populations and the assignment of individuals. Genetics Research 78:59-77

Dawson KJ, Belkhir K (2009) An agglomerative hierarchical approach to visualization in Bayesian clustering problems. Heredity 103:32-45

Diehl JM, Toonen RJ, Botsford LW (2007) Spatial variability of recruitment in the sand crab Emerita analoga throughout California in relation to wind-driven currents. Marine Ecology-Progress Series 350:1-17

Doherty PJ (1981) Coral Reef Fishes: Recruitment-Limited Assemblages? Proceedings of the Fourth International Coral Reef Symposium, Manila 2:465-670

Dupanloup I, Schneider S, Excoffier L (2002) A simulated annealing approach to define the genetic structure of populations. Mol Ecol 11:2571-2581

Elsdon T, Wells B, Campana S, Gillanders B, Jones C, Limburg K, Secor D, Thorrold S, Walther B (2008) Otolith Chemistry To Describe Movements And Life-History Parameters Of Fishes. In: Oceanography and Marine Biology, Vol null. CRC Press, p 297-330

Elsdon TS, Gillanders BM (2003) Reconstructing migratory patterns of fish based on environmental influences on otolith chemistry. Rev Fish Biol Fish 13:219-235

Elsdon TS, Gillanders BM (2004) Fish otolith chemistry influenced by exposure to multiple environmental variables. J Exp Mar Biol Ecol 313:269-284

Elsdon TS, Gillanders BM (2005) Consistency of patterns between laboratory experiments and field collected fish in otolith chemistry: an example and applications for salinity reconstructions. Mar Freshw Res 56:609-617

Elsdon TS, Gillanders BM (2006) Temporal variability in strontium, calcium, barium, and manganese in estuaries: Implications for 
reconstructing environmental histories of fish from chemicals in calcified structures. Estuarine Coastal Shelf Sci 66:147-156

Escobar MD, West M (1995) Bayesian Density Estimation and Inference Using Mixtures. J Amer Statistical Assoc 90:577-588

Fablet R, Daverat F, De Pontual H (2007) Unsupervised Bayesian reconstruction of individual life histories from otolith signatures: case study of $\mathrm{Sr}$ : Ca transects of European eel (Anguilla anguilla) otoliths. Can J Fish Aquat Sci 64:152-165

Feary DA, Clements KD (2006) Habitat use by triplefin species (Tripterygiidae) on rocky reefs in New Zealand. J Fish Biol 69:10311046

Ferguson T (1973) A Bayesian Analysis of Some Nonparametric Problems. The Annals of Statistics 1:209-230

Feyrer F, Hobbs J, Baerwald M, Sommer T, Yin QZ, Clark K, May B, Bennett W (2007) Otolith microchemistry provides information complementary to microsatellite DNA for a migratory fish. Trans Am Fish Soc 136:469-476

Fiksen O, Jorgensen C, Kristiansen T, Vikebo F, Huse G (2007) Linking behavioural ecology and oceanography: larval behaviour determines growth, mortality and dispersal. Marine Ecology-Progress Series 347:195-205

Fisher R (2005) Swimming speeds of larval coral reef fishes: impacts on self-recruitment and dispersal. Marine Ecology-Progress Series 285:223-232

Fogarty MJ, Botsford LW (2007) Population Connectivity and Spatial Management of Marine Fisheries. Oceanography 20:112-123

Fontes J, Caselle JE, Sheehy MS, Santos RS, Warner RR (2009) Natal signatures of juvenile Coris julis in the Azores: investigating connectivity scenarios in an oceanic archipelago. Mar Ecol Prog Ser 387:51-59

Forrester GE, Swearer SE (2002) Trace elements in otoliths indicate the use of open-coast versus bay nursery habitats by juvenile California halibut. Mar Ecol Prog Ser 241:201-213

Fowler AJ, Gillanders BM, Hall KC (2005) Relationship between elemental concentration and age from otoliths of adult snapper (Pagrus auratus, Sparidae): implications for movement and stock structure. Mar Freshw Res 56:661-676

Gaines SD, Gaylord B, Gerber LR, Hastings A, Kinlan BP (2007) Connecting Places The Ecological Consequences of Dispersal in the Sea. Oceanography 20:90-99

Gaylord B, Gaines SD (2000) Temperature or transport? Range limits in marine species mediated solely by flow. Am Nat 155:769-789

Gelman A, Carlin J, Stern H, Rubin D (2003) Bayesian Data Analysis, Second Edition (Chapman \& Hall/CRC Texts in Statistical Science), Vol. Chapman \& Hall

Gerlach G, Atema J, Kingsford MJ, Black KP, Miller-Sims V (2007) Smelling home can prevent dispersal of reef fish larvae. Proceedings of the National Academy of Sciences 104:858-863 
Gilg MR, Hilbish TJ (2003) The geography of marine larval dispersal: coupling genetics with fine-scale physical oceanography. Ecology 84:2989-2998

Gilks WR, Richardson S, Spiegelhalter DJ (1996) Markov chain Monte Carlo in practice, Vol. Chapman \& Hall, London ; New York

Gillanders BM (2002) Connectivity between juvenile and adult fish populations: do adults remain near their recruitment estuaries? Mar Ecol Prog Ser 240:215-223

Gillanders BM (2005) Using elemental chemistry of fish otoliths to determine connectivity between estuarine and coastal habitats. Estuarine Coastal Shelf Sci 64:47-57

Gillanders BM, Kingsford MJ (2000) Elemental fingerprints of otoliths of fish may distinguish estuarine 'nursery' habitats. Mar Ecol Prog Ser 201:273-286

Gouhier TC, Guichard F, Menge BA (2010) Ecological processes can synchronize marine population dynamics over continental scales. Proceedings of the National Academy of Sciences 107:8281-8286

Guenette S, Lauck T, Clark C (1998) Marine reserves: from Beverton and Holt to the present. Rev Fish Biol Fish 8:251-272

Guichard F, Bourget E, Robert J-L (2001) Scaling the influence of topographic heterogeniety on intertidal benthic communities: alternate trajectories mediated by hydrodynamics and shading. Mar Ecol Prog Ser 217:27-41

Guichard F, Levin SA, Hastings A, Siegel D (2004) Toward a dynamic metacommunity approach to marine reserve theory. Bioscience 54:1003-1011

Hale R, Swearer SE (2008) Otolith microstructural and microchemical changes associated with settlement in the diadromous fish Galaxias maculatus. Marine Ecology-Progress Series 354:229-234

Halpern BS, Regan HM, Possingham HP, McCarthy MA (2006) Accounting for uncertainty in marine reserve design. Ecol Lett 9:211

Hamilton SL, Regetz J, Warner RR (2008) Postsettlement survival linked to larval life in a marine fish. Proc Natl Acad Sci U S A 105:1561-1566

Hamilton SL, Warner RR (2009) Otolith barium profiles verify the timing of settlement in a coral reef fish. Marine Ecology-Progress Series 385:237-244

Hanski I (1999) Metapopulation Ecology, Vol. Oxford University Press

Hastie T, Tibshirani R (1996) Discriminant Analysis by Gaussian Mixtures. Journal of the Royal Statistical Society Series B (Methodological) 58:155-176

Hastings A, Botsford LW (1999) Equivalence in yield from marine reserves and traditional fisheries management. Science 284:1537-1538

Hastings A, Botsford LW (2006) Persistence of spatial populations depends on returning home. Proc Natl Acad Sci U S A 103:6067-6072

Hauser L, Seamons TR, Dauer M, Naish KA, Quinn TP (2006) An empirical verification of population assignment methods by marking 
and parentage data: hatchery and wild steelhead (Oncorhynchus mykiss) in Forks Creek, Washington, USA. Mol Ecol 15:3157-3173

Hawkes C (2009) Linking movement behaviour, dispersal and population processes: is individual variation a key? J Anim Ecol 78:894-906

Heath RA (1985) A review of the physical oceanography of the seas around New Zealand - 1982. N Z J Mar Freshwater Res 19:79-124

Heath RA (1986) In which direction is the mean flow through Cook Strait, New Zealand - evidence of 1 to 4 week variability. N Z J Mar Freshwater Res 20:119-137

Hedger RD, Atkinson PM, Thibault I, Dodson JJ (2008) A quantitative approach for classifying fish otolith strontium: Calcium sequences into environmental histories. Ecol Inform 3:207-217

Heller KA, Ghahramani Z (2005) Bayesian hierarchical clustering Proceedings of the 22nd international conference on Machine learning. ACM, Bonn, Germany, p 297-304

Hill MF, Hastings A, Botsford LW (2002) The effects of small dispersal rates on extinction times in structured metapopulation models. Am Nat 160:389-402

Hobson KA (1999) Tracing origins and migration of wildlife using stable isotopes: a review. Oecologia 120:314-326

Huelsenbeck JP, Andolfatto P (2007) Inference of population structure under a Dirichlet process model. Genetics 175:1787-1802

Hutchings JA (2000) Collapse and recovery of marine fishes. Nature 406:882-885

Jain S, Neal RM (2004) A split-merge Markov chain Monte Carlo procedure for the dirichlet process mixture model. Journal of Computational and Graphical Statistics 13:158-182

James MK, Armsworth PR, Mason LB, Bode L (2002) The structure of reef fish metapopulations: modelling larval dispersal and retention patterns. Proceedings of the Royal Society of London Series BBiological Sciences 269:2079-2086

Jax K, Jones CG, Pickett STA (1998) The self-identity of ecological units. Oikos 82:253-264

Jones GP, Milicich MJ, Emslie MJ, Lunow C (1999) Self-recruitment in a coral reef fish population. Nature 402:802-804

Jordan MI (2004) Graphical models. Statistical Science 19:140-155

Kass RE, Raftery AE (1995) Bayes Factors. J Amer Statistical Assoc 90:773-795

Kelly L, Stefan L (2007) New Zealand's Quota Management System: A History of the First 20 Years, Motu Economic and Public Policy Research

Kendall BE, Bjoernstad ON, Bascompte J, Keitt TH, Fagan WF (2000) Dispersal, Environmental Correlation, and Spatial Synchrony in Population Dynamics. The American Naturalist 155:628-636

Kingsford MJ, Leis JM, Shanks A, Lindeman KC, Morgan SG, Pineda J (2002) Sensory environments, larval abilities, and local selfrecruitment. Bull Mar Sci 70:309-340 
Koehl MAR, Strother JA, Reidenbach MA, Koseff JR, Hadfield MG (2007) Individual-based model of larval transport to coral reefs in turbulent, wave-driven flow: behavioral responses to dissolved settlement inducer. Mar Ecol Prog Ser 335:1-18

Kohn Y (2007) Pelagic larval duration in New Zealand triplefin fishes. University of Auckland

Koljonen ML, Pella JJ, Masuda M (2005) Classical individual assignments versus mixture modeling to estimate stock proportions in Atlantic salmon (Salmo salar) catches from DNA microsatellite data. Can J Fish Aquat Sci 62:2143-2158

Kritzer JP, Sale PF (2004) Metapopulation ecology in the sea: from Levins' model to marine ecology and fisheries science. Fish Fish 5:131-140

Largier JL (2003) Considerations in estimating larval dispersal distances from oceanographic data. Ecol Appl 13:S71-S89

Lecchini D (2005) Spatial and behavioural patterns of reef habitat settlement by fish larvae. Marine Ecology-Progress Series 301:247252

Lecchini D, Shima J, Banaigs B, Galzin R (2005) Larval sensory abilities and mechanisms of habitat selection of a coral reef fish during settlement. Oecologia 143:326-334

Leis J, Stobutzki I (1999) Swimming performance of late pelagic larvae of coral-reef fishes: in situ and laboratory-based measurments. Proceedings from the 5th Indo-Pacific Fish Conference, Noumea 1999:1-9

Leis JM (2007) Behaviour as input for modelling dispersal of fish larvae: behaviour, biogeography, hydrodynamics, ontogeny, physiology, and phylogeny meet hydrography. Mar Ecol Prog Ser 347:185-193

Leis JM, Carson-Ewart BM (2003) Orientation of pelagic larvae of coralreef fishes in the ocean. Mar Ecol Prog Ser 252:239-253

Levin LA (2006) Recent progress in understanding larval dispersal: new directions and digressions. Integr Comp Biol 46:282-297

Levins R (1969) Some demographic and genetic consequences of environmental heterogeniety for biological control. Bull Entomol Soc Am 15:237-240

Lockwood DR, Hastings A (2002) The effects of dispersal patterns on marine reserves: does the tail wag the dog? Theor Popul Biol 61:297-309

Lunn DJ, Thomas A, Best N, Spiegelhalter D (2000) WinBUGS - A Bayesian modelling framework: Concepts, structure, and extensibility. Statistics and Computing 10:325-337

Mace AJ, Morgan SG (2006) Larval accumulation in the lee of a small headland: implications for the design of marine reserves. Marine Ecology-Progress Series 318:19-29

Manel S, Gaggiotti OE, Waples RS (2005) Assignment methods: matching biological questions techniques with appropriate. Trends Ecol Evol 20:136-142

Marine_Zooplankton_Colloquium_2 (2001) Future marine zooplankton research--a perspective. Mar Ecol Prog Ser 222:297-308 
Marshall DJ, Allen RM, Crean AJ (2008) The ecological and evolutionary importance of maternal effects in the sea. In: Oceanography and Marine Biology: an Annual Review, Vol 46, Vol 46. Crc PressTaylor \& Francis Group, Boca Raton, p 203-250

Marshall DJ, Monro K, Bode M, Keough MJ, Swearer S (2010) Phenotypeenvironment mismatches reduce connectivity in the sea. Ecol Lett $13: 128-140$

Mercier L, Darnaude A, Bruguier O, Vasconcelos R, Costa M, Cabral H, Lara M, Jones D, Mouillot D (in press) Selecting statistical models and variable combinations for optimal classification using otolith microchemistry. Ecol Appl

Millar RB (1987) Maximum likelihood estiamtion of mixed stock fishery composition. Can J Fish Aquat Sci 44:583-590

Miller JA (2007a) Scales of variation in otolith elemental chemistry of juvenile staghorn sculpin (Leptocottus armatus) in three Pacific Northwest estuaries. Mar Biol 151:483-494

Miller JA, Gray A, Merz J (2010) Quantifying the contribution of juvenile migratory phenotypes in a population of Chinook salmon Oncorhynchus tshawytscha. Mar Ecol Prog Ser 408:227-240

Miller TJ (2007b) Contribution of individual-based coupled physicalbiological models to understanding recruitment in marine fish populations. Mar Ecol Prog Ser 347:127-138

Mitarai S, Siegel DA, Winters KB (2008) A numerical study of stochastic larval settlement in the California Current system. J Mar Syst 69:295-309

Morel FMM, Price NM (2003) The biogeochemical cycles of trace metals in the oceans. Science 300:944-947

Morgan SG, Anastasia JR (2008) Behavioral tradeoff in estuarine larvae favors seaward migration over minimizing visibility to predators. Proceedings of the National Academy of Sciences 105:222-227

Morgan SG, Fisher JL (2010) Larval behavior regulates nearshore retention and offshore migration in an upwelling shadow and along the open coast. Mar Ecol Prog Ser 404:109-126

Msadek R (2005) Hydrodynamic tidal model of Cook Strait, National Institute of Water and Atmospheric Research, Wellington, NZ

Munch SB, Clarke LM (2008) A Bayesian approach to identifying mixtures from otolith chemistry data. Can J Fish Aquat Sci 65:2742-2751

Nahas EL, Jackson G, Pattiaratchi CB, Ivey GN (2003) Hydrodynamic modelling of snapper Pagrus auratus egg and larval dispersal in Shark Bay, Western Australia: reproductive isolation at a fine spatial scale. Marine Ecology-Progress Series 265:213-226

Neal R (1992) Bayesian Mixture Modeling. In: Smith CR, Erickson GJ, Neudorfer PO (eds) Maximum Entropy and Bayesian Methods: Proceedings of the 11th International Workshop on Maximum Entropy and Bayesian Methods of Statistical Analysis, Seattle, 1991. Kluwer Academic Publishers, Dordrecht, p 197-211 
Neal R (2000) Markov Chain Sampling Methods for Dirichlet Process Mixture Models. Journal of Computational and Graphical Statistics 9:249-265

Neubauer P, Hadfield M, Delaux S, Popinet S, Shima JS (in prep) Validation of an adaptive grid ocean model for simulation of larval dispersal in Cook Strait, New Zealand.

Neubauer P, Shima JS, Swearer SE (2010) Scale-dependent variability in natal otolith chemistry: implications and solutions for studies of population connectivity. Mar Ecol Prog Ser 415:263-274

Ohlssen DI, Sharples LD, Spiegelhalter DJ (2007) Flexible random-effects models using Bayesian semi-parametric models: applications to institutional comparisons. Stat Med 26:2088-2112

Palumbi SR (1994) Genetic divergence, reproductive isolation, and marine speciation. Annu Rev Ecol Syst 25:547-572

Palumbi SR (2003) Population genetics, demographic connectivity, and the design of marine reserves. Ecol Appl 13:S146-S158

Paris CB, Cowen RK (2004) Direct evidence of a biophysical retention mechanism for coral reef fish larvae. Limnol Oceanogr 49:19641979

Patterson TA, Basson M, Bravington MV, Gunn JS (2009) Classifying movement behaviour in relation to environmental conditions using hidden Markov models. J Anim Ecol 78:1113-1123

Patterson TA, Thomas L, Wilcox C, Ovaskainen O, Matthiopoulos J (2008) State-space models of individual animal movement. Trends Ecol Evol 23:87-94

Pauly D, Christensen V, Guenette S, Pitcher TJ, Sumaila UR, Walters CJ, Watson R, Zeller D (2002) Towards sustainability in world fisheries. Nature 418:689-695

Peck MA, Daewel U (2007) Physiologically based limits to food consumption, and individual-based modeling of foraging and growth of larval fishes. Mar Ecol Prog Ser 347:171-183

Pella J, Masuda M (2001) Bayesian methods for analysis of stock mixtures from genetic characters. Fish Bull 99:151-167

Pella J, Masuda M (2006) The Gibbs and split-merge sampler for population mixture analysis from genetic data with incomplete baselines. Can J Fish Aquat Sci 63:576-596

Pikitch EK, Santora C, Babcock EA, Bakun A, Bonfil R, Conover DO, Dayton P, Doukakis P, Fluharty D, Heneman B, Houde ED, Link J, Livingston PA, Mangel M, McAllister MK, Pope J, Sainsbury KJ (2004) ECOLOGY: Ecosystem-Based Fishery Management. Science 305:346-347

Planes S, Jones GP, Thorrold SR (2009) Larval dispersal connects fish populations in a network of marine protected areas. Proc Natl Acad Sci U S A 106:5693-5697

Popinet S (2003) Gerris: a tree-based adaptive solver for the incompressible Euler equations in complex geometries. Journal of Computational Physics 190:572-600 
Popinet S, Rickard G (2007) A tree-based solver for adaptive ocean modelling. Ocean Model Online 16:224-249

Roberts CM (1997) Connectivity and management of Caribbean coral reefs. Science 278:1454-1457

Roberts CM, Bohnsack JA, Gell F, Hawkins JP, Goodridge R (2001) Effects of marine reserves on adjacent fisheries. Science 294:1920-1923

Royer T, Fromentin J-M, Gaspar P (2005) A state-space model to derive bluefin tuna movement and habitat from archival tags. Oikos 109:473-484

Rozenfeld AF, Arnaud-Haond S, HernÃ $i n d e z-G a r c \tilde{A}-a$ E, EguÃ-luz VcM, SerrÃ£o EA, Duarte CM (2008) Network analysis identifies weak and strong links in a metapopulation system. Proceedings of the National Academy of Sciences 105:18824-18829

Rubenstein DR, Chamberlain CP, Holmes RT, Ayres MP, Waldbauer JR, Graves GR, Tuross NC (2002) Linking Breeding and Wintering Ranges of a Migratory Songbird Using Stable Isotopes. Science 295:1062-1065

Rubenstein DR, Hobson KA (2004) From birds to butterflies: animal movement patterns and stable isotopes. Trends Ecol Evol 19:256263

Ruttenberg BI, Hamilton SL, Hickford MJH, Paradis GL, Sheehy MS, Standish JD, Ben-Tzvi O, Warner RR (2005) Elevated levels of trace elements in cores of otoliths and their potential for use as natural tags. Mar Ecol Prog Ser 297:273-281

Ruttenberg BI, Hamilton SL, Warner RR (2008) Spatial and temporal variation in the natal otolith chemistry of a Hawaiian reef fish: prospects for measuring population connectivity. Can J Fish Aquat Sci 65:1181-1192

Ruttenberg BI, Warner RR (2006) Spatial variation in the chemical composition of natal otoliths from a reef fish in the Galapagos Islands. Mar Ecol Prog Ser 328:225-236

Ruzzante DE, Mariani S, Bekkevold D, Andre C, Mosegaard H, Clausen LAW, Dahlgren TG, Hutchinson WF, Hatfield EMC, Torstensen E, Brigham J, Simmonds EJ, Laikre L, Larsson LC, Stet RJM, Ryman N, Carvalho GR (2006) Biocomplexity in a highly migratory pelagic marine fish, Atlantic herring. Proceedings of the Royal Society BBiological Sciences 273:1459-1464

Sale P (1998) Appropriate spatial scales for studies of reef-fish ecology. Aust J Ecol 23:202-208

Sale PF, Cowen RK, Danilowicz BS, Jones GP, Kritzer JP, Lindeman KC, Planes S, Polunin NVC, Russ GR, Sadovy YJ, Steneck RS (2005) Critical science gaps impede use of no-take fishery reserves. Trends Ecol Evol 20:74-80

Sandin SA, Regetz J, Hamilton SL (2005) Testing larval fish dispersal hypotheses using maximum likelihood analysis of otolith chemistry data. Mar Freshw Res 56:725-734

Schaefer JA (2006) Towards maturation of the population concept. Oikos $112: 236-240$ 
Schick RS, Loarie SR, Colchero F, Best BD, Boustany A, Conde DA, Halpin PN, Joppa LN, McClellan CM, Clark JS (2008) Understanding movement data and movement processes: current and emerging directions. Ecol Lett 11:1338-1350

Schnute JT, Richards LJ (2001) Use and abuse of fishery models. Can J Fish Aquat Sci 58:10-17

Scott SL (2002) Bayesian methods for hidden Markov models: Recursive computing in the 21st century. J Amer Statistical Assoc 97

Secor D, Piccoli P (1996) Age- and sex-dependent migrations of striped bass in the Hudson River as determined by chemical microanalysis of otoliths. Estuar Coasts 19:778-793

Secor DH (1999) Specifying divergent migrations in the concept of stock: the contingent hypothesis. Fisheries Research 43:13-34

Shanks AL (2009) Pelagic Larval Duration and Dispersal Distance Revisited. Biol Bull 216:373-385

Shanks AL, Eckert GL (2005) Population persistence of California Current fishes and benthic crustaceans: A marine drift paradox. Ecol Monogr 75:505-524

Shanks AL, Grantham BA, Carr MH (2003) Propagule dispersal distance and the size and spacing of marine reserves. Ecol Appl 13:S159S169

Shanks AL, Kipp Shearman R (2009) Paradigm lost? Cross-shelf distributions of intertidal invertebrate larvae are unaffected by upwelling or downwelling. Mar Ecol Prog Ser 385:189-204

Shima JS, Swearer SE (2009a) Larval quality is shaped by matrix effects: implications for connectivity in a marine metapopulation. Ecology 90:1255-1267

Shima JS, Swearer SE (2009b) Spatially variable larval histories may shape recruitment rates of a temperate reef fish. Marine Ecology-Progress Series 394:223-229

Shima JS, Swearer SE (2010) The legacy of dispersal: larval experience shapes persistence later in the life of a reef fish. J Anim Ecol 79:1308-1314

Siegel DA, Kinlan BP, Gaylord B, Gaines SD (2003) Lagrangian descriptions of marine larval dispersion. Mar Ecol Prog Ser 260:8396

Siegel DA, Mitarai S, Costello CJ, Gaines SD, Kendall BE, Warner RR, Winters KB (2008) The stochastic nature of larval connectivity among nearshore marine populations. Proc Natl Acad Sci U S A 105:8974-8979

Smedbol RK, Wroblewski JS (2002) Metapopulation theory and northern cod population structure: interdependency of subpopulations in recovery of a groundfish population. Fisheries Research 55:161-174

Smith ANH (2008) Predicting the distribution and relative abundance of fishes on shallow subtidal reefs around New Zealand, NIWA, Wellington 
Smith SJ, Campana SE (2010) Integrated stock mixture analysis for continous and categorical data, with application to genetic-otolith combinations. Can J Fish Aquat Sci 67:1533-1548

Smouse PE, Spielman RS, Park MH (1982) Multiple-locus allocation of individuals to groups as a function of the genetic variation within and differences among human populations. Am Nat 119:445-463

Standish JD, Sheehy M, Warner RR (2008) Use of otolith natal elemental signatures as natural tags to evaluate connectivity among open-coast fish populations. Mar Ecol Prog Ser 356:259-268

Stobutzki IC (2001) Marine reserves and the complexity of larval dispersal. Rev Fish Biol Fish 10:515-518

Strathmann RR, Hughes TP, Kuris AM, Lindeman KC, Morgan SG, Pandolfi JM, Warner RR (2002) Evolution of local recruitment and its consequences for marine populations. Bull Mar Sci 70:377-396

Swearer S, Caselle J, Lea D, Warner R (1999) Larval retention and recruitment in an island population of a coral reef fish. Nature 402:799-802

Swearer SE, Shima JS (2010) Regional variation in larval retention and dispersal drives recruitment patterns in a temperate reef fish. Mar Ecol Prog Ser 417:229-236

Swearer SE, Shima JS, Helberg ME, Thorrold SR, Jones GP, Robertson DR, Morgan SG, Selkoe KA, Ruiz GM, Warner RR (2002) Evidence of self-recruitment in demersal marine populations. Bull Mar Sci 70:251-271

Szép T, Møller AP, Vallner J, Kovács B, Norman D (2003) Use of trace elements in feathers of sand martin Riparia riparia for identifying moulting areas. J Avian Biol 34:307-320

Taylor MS, Hellberg ME (2003) Genetic evidence for local retention of pelagic larvae in a Caribbean reef fish. Science 299:107-109

Team RDC (2007) R: A language and environment for statistical computing. R Foundation for Statistical Computing. R Foundation for Statistical Computing, Vienna, Austria

Thorrold S, Jones C, Swart P, Targett T (1998) Acurate classification of juvenile weakfish Cynoscion regalis to estuarine nursery areas based on chemical signatures in otoliths. Mar Ecol Prog Ser 173:253-265

Thorrold SR, Jones GP, Helberg ME, Burton RS, Swearer SE, Neigel JE, Morgan SG, Warner RR (2002) Quantifying larval retention and connectivity in marine populations with artificial and natural markers. Bull Mar Sci 70:291-308

Thorrold SR, Jones GP, Planes S, Hare JA, Dt (2006) Transgenerational marking of embryonic otoliths in marine fishes using barium stable isotopes. Can J Fish Aquat Sci 63:1193-1197

Thorrold SR, Latkoczy C, Swart PK, Jones CM (2001) Natal homing in a marine fish metapopulation. Science 291:297-299

Treml EA, Halpin PN, Urban DL, Pratson LF (2008) Modeling population connectivity by ocean currents, a graph-theoretic approach for marine conservation. Landsc Ecol 23:19-36 
Walther BD, Thorrold SR (2006) Water, not food, contributes the majority of strontium and barium deposited in the otoliths of a marine fish. Mar Ecol Prog Ser 311:125-130

Waples RS, Gaggiotti O (2006) What is a population? An empirical evaluation of some genetic methods for identifying the number of gene pools and their degree of connectivity. Mol Ecol 15:1419-1439

Warner RR, Swearer SE, Caselle JE, Sheehy M, Paradis G (2005) Natal trace-elemental signatures in the otoliths of an open-coast fish. Limnol Oceanogr 50:1529-1542

Wellenreuther M, Barrett PT, Clements KD (2007) Ecological diversification in habitat use by subtidal triplefin fishes (Tripterygiidae). Mar Ecol Prog Ser 330:235-246

Wellenreuther M, Clements KD (2007) Reproductive isolation in temperate reef fishes. Mar Biol

White C, Kendall BE (2007) A reassessment of equivalence in yield from marine reserves and traditional fisheries managament. Oikos 116:2039-2043

White JW, Botsford LW, Hastings A, Largier JL (2010) Population persistence in marine reserve networks: incorporating spatial heterogeneities in larval dispersal. Mar Ecol Prog Ser 398:49-67

White JW, Ruttenberg BI (2006) Discriminant function analysis in marine ecology: some oversights and their solutions. Mar Ecol Prog Ser 329:301-305

White JW, Standish JD, Thorrold SR, Warner RR (2008) Makov Chain Monte Carlo Methods for assigning larvae to natal sites using natural geochemical tags. Ecol Appl 18:1901-1913

Zacherl DC (2005) Spatial and temporal variation in statolith and protoconch trace elements as natural tags to track larval dispersal. Marine Ecology-Progress Series 290:145-163

Zimmer RK, Fingerut JT, Zimmer CA (2009) Dispersal pathways, seed rains, and the dynamics of larval behavior. Ecology 90:1933-1947 


\section{APPENDIX}

\section{A.1 Weakfish exact linkage tree from the DPM clustering model}

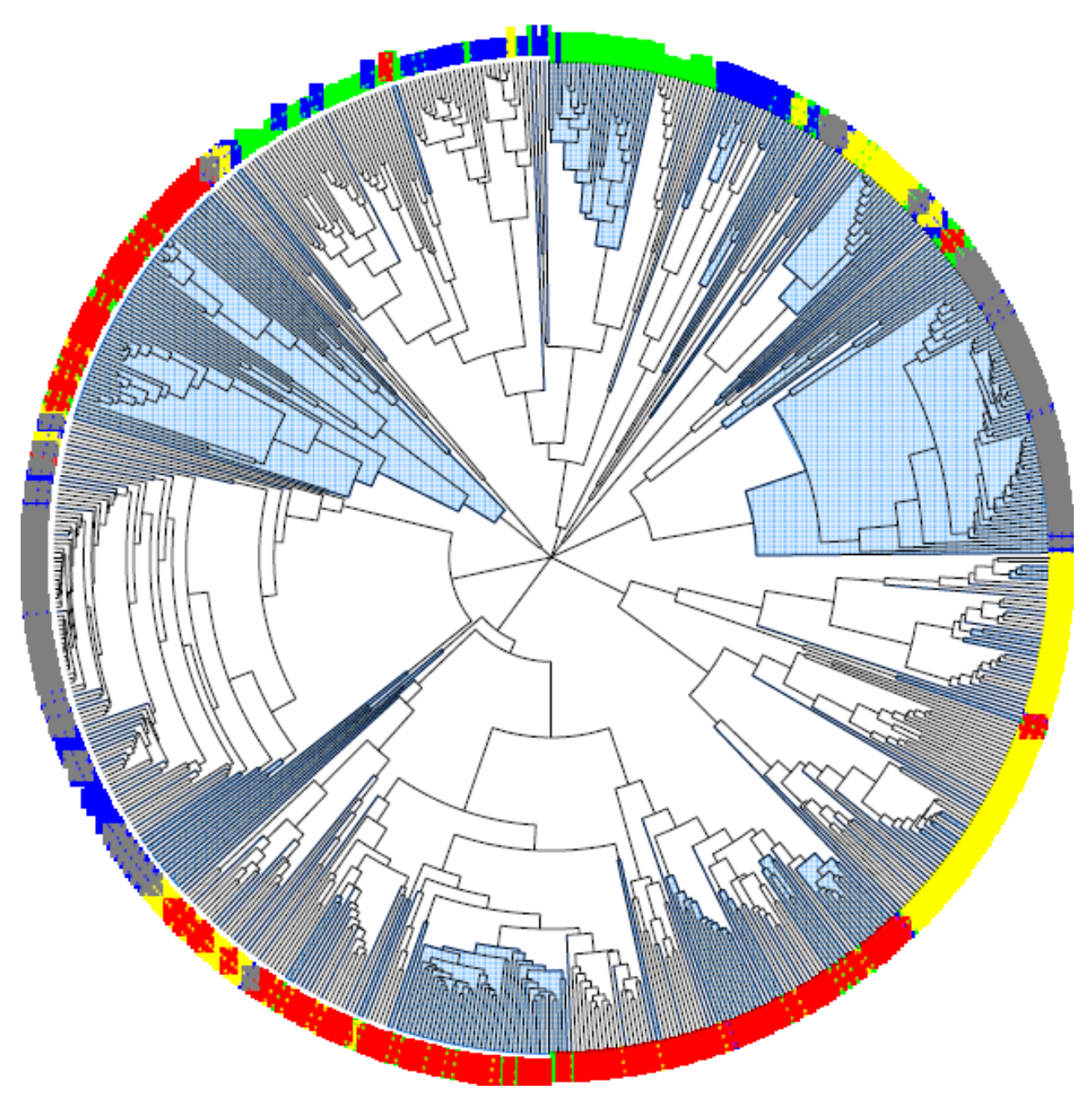

Figure A1: Weakfish exact linkage tree from the DPM clustering model with the baseline clustered together with the adult mixed sample. Sub-trees including adult weakfish are highlighted in light blue. Leaf colours indicate collection location (North to South): New York (grey), Delaware (blue), Chesapeake Bay (green), North Carolina (red) and Georgia (yellow). Fish Id numbers in the tree leafs were retained to visualize heterogeneity in clusters. 


\section{A.2 Gibbs sampling in the mixture models}

The relevant conditional posteriors are given here for a two component random effects mixture model discussed in chapter 5 , from which those of easier models can be readily obtained by omitting the random effects. I then give the procedure to sample the state sequence from the HMM in appendix A.3. Some posteriors (those involving T) require slight modification if transects have an unequal number of observations.

1. Start by drawing $\xi_{i, t}$ at iteration $\mathrm{s}=1$ of the Gibbs sampler, conditional on all other parameters as

$$
\begin{aligned}
\xi_{i, t} \mid \pi_{i}, \theta & \sim \operatorname{Bernoulli}\left(z_{i, t}\right) \\
z_{i, t_{s}} & =\frac{\pi_{i} N\left(y_{i, t} \mid \mu+\nu_{i}, \sigma_{\nu}^{2}\right)}{\pi_{i} N\left(y_{i, t} \mid \mu+\nu_{i}, \sigma_{\nu}^{2}\right)+\left(1-\pi_{i}\right) N\left(y_{i, t} \mid \mu, \sigma_{\mu}^{2}\right)}
\end{aligned}
$$

2. I then update the mixture proportions (for more than one class this becomes a Dirichlet distribution.

$$
\pi_{i} \mid \zeta \sim \operatorname{Beta}\left(1+\sum_{t=1}^{T} \xi_{i, t}, 1+\left(T-\sum_{t=1}^{T} \xi_{i, t}\right)\right)
$$

3. Draw $\mu$ as

$$
\mu \mid \theta, \zeta \sim N\left(\frac{\sum_{i=1}^{N} \sum_{t=1}^{T} y_{i, t}-\xi_{i, t} \eta_{i}}{N T}, \frac{\sigma_{\mu}^{2}}{N T}\right)
$$

4. The random effects $\eta$ are updated as

$$
\eta_{i} \mid \theta, \xi_{i} \sim N\left(\frac{\frac{1}{\varsigma^{2}} \alpha+\sum_{t=1}^{T} \frac{\xi_{i, t}}{\sigma_{\nu}^{2}}\left(y_{i, t}-\mu\right)}{\frac{1}{\varsigma^{2}}+\sum_{t=1}^{T} \frac{1}{\sigma_{\nu}^{2}}}, \frac{1}{\frac{1}{\varsigma^{2}}+\sum_{t=1}^{T} \frac{1}{\sigma_{\nu}^{2}}}\right)
$$

5. Next I update the mean of the random effects

$$
\alpha \mid \theta \sim N\left(\frac{\sum_{i=1}^{N} \eta_{i}}{N}, \frac{\varsigma^{2}}{N}\right)
$$


6. Lastly, the three variances are updated

$$
\begin{aligned}
\sigma_{\mu}^{2} \mid \theta, \xi & \sim i n v-\chi^{2}\left(\frac{1}{\left(N T-\sum_{i=1}^{N} \sum_{t=1}^{T} \xi_{i, t}\right)-2}, \sum_{i=1}^{N} \sum_{t=1}^{T}\left(1-\xi_{i, t}\right)\left(y_{i, t}-\mu\right)^{2}\right) \\
\sigma_{\nu}^{2} \mid \theta, \xi & \sim i n v-\chi^{2}\left(\frac{1}{\left(\sum_{i=1}^{N} \sum_{t=1}^{T} \xi_{i, t}\right)-2}, \sum_{i=1}^{N} \sum_{t=1}^{T} \xi_{i, t}\left(y_{i, t}-\mu-\eta_{i}\right)^{2}\right) \\
\varsigma^{2} \mid \theta, \xi & \sim i n v-\chi^{2}\left(\frac{1}{N-2}, \sum_{i=1}^{N}\left(\alpha-\eta_{i}\right)^{2}\right)
\end{aligned}
$$

\section{A.3 Drawing a state sequence from the HMM - the recursive method}

Steps 1 and 2 of the sampler for the mixture model in A.2 need to be modified in order to estimate the HMM. The likelihood for the HMM can be written as:

$f(y \mid \theta, \pi)=\prod_{i=1}^{N} \prod_{t=1}^{T} f\left(y_{i, t} \mid \mathbf{y}_{\mathbf{i}, \mathbf{t}-\mathbf{1}}, \theta\right)$

Where $\mathbf{y}_{\mathbf{i}, \mathbf{t}-\mathbf{1}}$ is the observed data up to scan $\mathbf{t}$, and

$f\left(y_{i, t} \mid \mathbf{y}_{\mathbf{i}, \mathbf{t}-\mathbf{1}}, \theta\right)=\sum_{\mathbf{k}=\mathbf{1}}^{\mathbf{K}} \mathbf{p}\left(\xi_{\mathbf{i}, \mathbf{t}}=\mathbf{k} \mid \mathbf{y}_{\mathbf{i}, \mathbf{t}-\mathbf{1}}, \theta\right) \mathbf{N}\left(\mathbf{y}_{\mathbf{i}, \mathbf{t}} \mid \theta_{\mathbf{k}}\right)$.

Gibbs sampling from the posterior in the HMM requires the calculation of this likelihood, which can be efficiently achieved with a recursive algorithm (Scott 2002). The algorithm consists of a forward step from 1 to $T$ and a backward step from $T$ to 1 . The forward step gives the likelihood while the backwards step calculates the probabilities for the state sequence. Specifically,

Forward step: for $\mathrm{t}=1 \ldots$ T calculate

$$
p\left(\xi_{i, t}=k \mid \mathbf{y}_{\mathbf{i}, \mathbf{t}-\mathbf{1}}, \theta\right)=\sum_{\mathbf{j}=\mathbf{1}}^{\mathbf{K}} \pi_{\mathbf{k} \mid \mathbf{j}}^{\mathbf{i}} \mathbf{p}\left(\xi_{\mathbf{i}, \mathbf{t}-\mathbf{1}}=\mathbf{j} \mid \boldsymbol{y}_{\mathbf{i}, \mathbf{t}-\mathbf{1}}, \theta\right)
$$


From this compute the following probabilities

$p\left(\xi_{i, t}=k \mid \mathbf{y}_{\mathbf{i}, \mathbf{t}}, \theta\right) \propto \mathbf{p}\left(\xi_{\mathbf{i}, \mathbf{t}}=\mathbf{k} \mid \mathbf{y}_{\mathbf{i}, \mathbf{t}-\mathbf{1}}, \theta\right) * \mathbf{N}\left(\mathbf{y}_{\mathbf{i}, \mathbf{t}} \mid \theta_{\mathbf{k}}\right)$

Backwards step: for $\mathrm{t}=\mathrm{T}, . ., 1$

First, draw $\xi_{i, T}$ from $p\left(\xi_{i, T}=k \mid \mathbf{y}_{\mathbf{i}, \mathbf{T}}, \theta\right)$. Then update the remaining probabilities according to:

$p\left(\xi_{i, t}=k \mid \xi_{i, t+1}=j, \mathbf{y}_{\mathbf{i}, \mathbf{T}}, \theta\right) \propto \pi_{\mathbf{k} \mid \mathbf{j}}^{\mathbf{i}} * \mathbf{p}\left(\xi_{\mathbf{i}, \mathbf{t}}=\mathbf{k} \mid \mathbf{y}_{\mathbf{i}, \mathbf{t}}, \theta\right)$

These probabilities are then use to draw the remaining $\xi_{i, T-1}$.

Similar to the Gibbs sampler for the mixture model, the beta/Dirichlet distribution can be used to simulate from the transition matrix. The sum over observed scans in a given state (i.e. $\sum_{t=1}^{T} \xi_{i, t}$ and $\mathrm{T}-\sum_{t=1}^{T} \xi_{i, t}$ in the two component model) is then replaced by the sum of observed transitions per row (=per state), and each row is simulated independently from the beta/Dirichlet distribution.

\section{A.4 Winbugs code}

I give here the WinBUGS/ OpenBUGS code for a mixture model with random effects, and hierarchical prior on mixing proportions $\pi$ as discussed in chapter 5.1 provide this model since all other models can be readily obtained by omitting or moving relevant parameters, as indicated in the code. The code is written to accommodate transects of different lengths. The data needs to be in a format where the first column indicates the fish number $(1 \ldots \mathrm{N})$ and the second column gives the scans. Note that all variances are parameterized as their inverse (precision). The code was tested in OpenBUGS. 


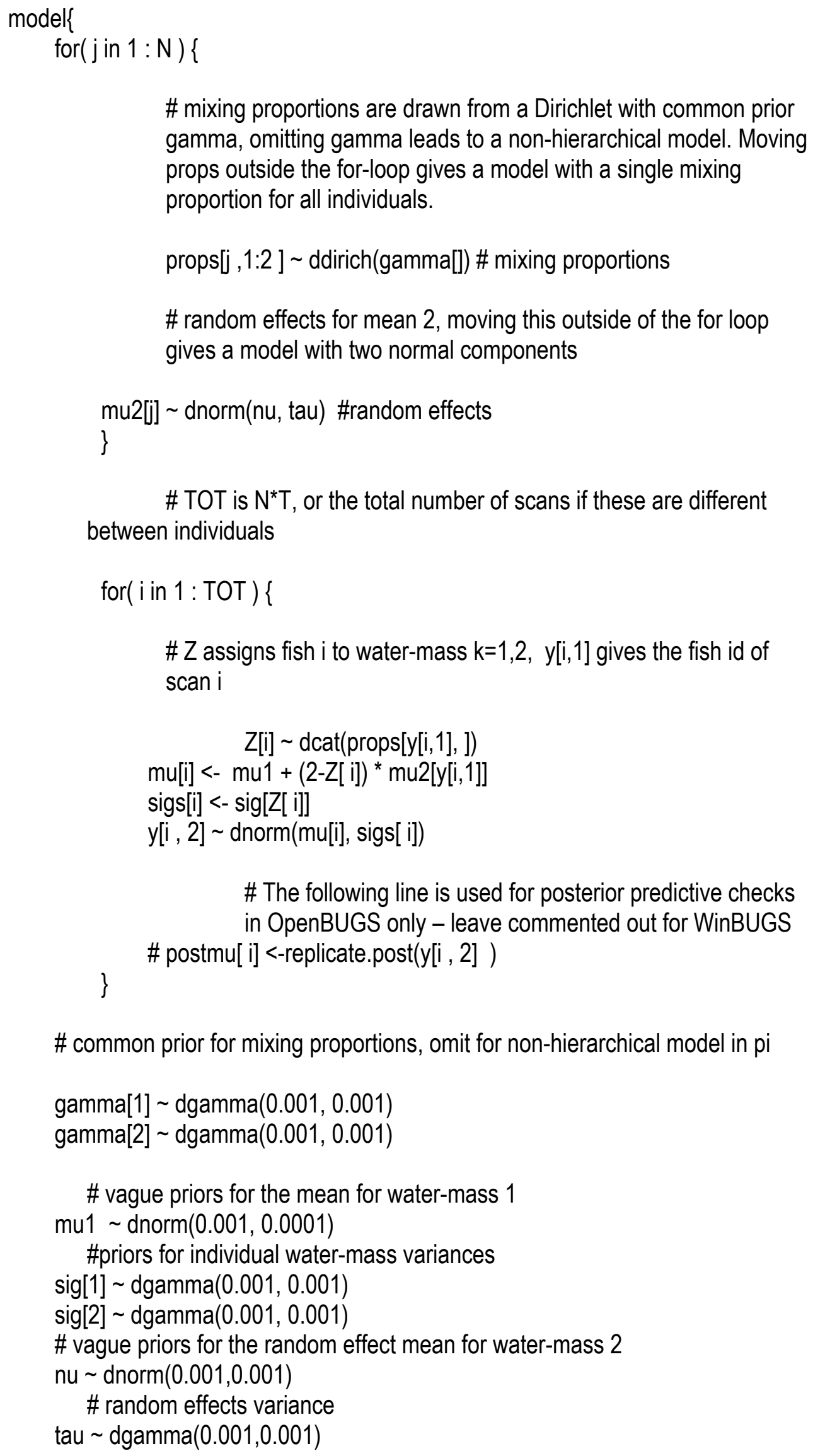

\# TOT is $\mathrm{N}^{*} \mathrm{~T}$, or the total number of scans if these are different between individuals

\# $Z$ assigns fish $i$ to water-mass $k=1,2, y[i, 1]$ gives the fish id of scan i

$$
\mathrm{Z}[\mathrm{i}] \sim \operatorname{dcat}(\operatorname{props}[\mathrm{y}[\mathrm{i}, 1], \mathrm{]})
$$$$
m u[i]<-m u 1+(2-Z[i]){ }^{*} m u 2[y[i, 1]]
$$$$
\text { sigs[i] }<-\operatorname{sig}[Z[i]]
$$$$
y[i, 2] \sim \operatorname{dnorm}(\mathrm{mu}[\mathrm{i}], \operatorname{sigs}[\mathrm{i}])
$$

\# The following line is used for posterior predictive checks in OpenBUGS only - leave commented out for WinBUGS \# postmu[ i] <-replicate.post(y[i , 2] )

\# common prior for mixing proportions, omit for non-hierarchical model in pi 